OAK RIDGE

NATIONAL LABORATORY

\title{
Simulating High Flux Isotope Reactor Core Thermal-Hydraulics via Interdimensional Model Coupling
}

May 2014

\author{
A. R. Travis \\ K. Ekici \\ J. D. Freels
}

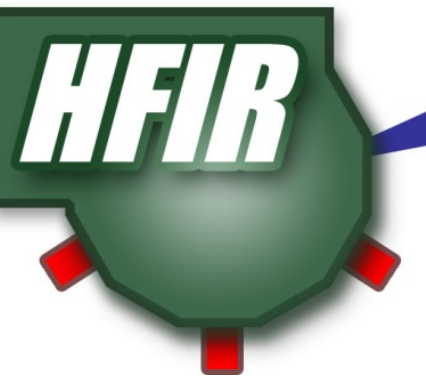

Approved for public release; distribution is unlimited.

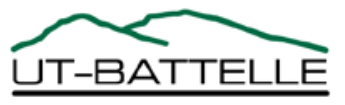




\section{DOCUMENT AVAILABILITY}

Reports produced after January 1, 1996, are generally available free via the U.S. Department of Energy (DOE) Information Bridge.

Web site http://www.osti.gov/bridge

Reports produced before January 1, 1996, may be purchased by members of the public from the following source.

National Technical Information Service

5285 Port Royal Road

Springfield, VA 22161

Telephone 703-605-6000 (1-800-553-6847)

TDD 703-487-4639

Fax 703-605-6900

E-mail info@ntis.gov

Web site http://www.ntis.gov/support/ordernowabout.htm

Reports are available to DOE employees, DOE contractors, Energy Technology Data Exchange (ETDE) representatives, and International Nuclear Information System (INIS) representatives from the following source.

Office of Scientific and Technical Information

P.O. Box 62

Oak Ridge, TN 37831

Telephone 865-576-8401

Fax 865-576-5728

E-mail reports@osti.gov

Web site http://www.osti.gov/contact.html

This report was prepared as an account of work sponsored by an agency of the United States Government. Neither the United States Government nor any agency thereof, nor any of their employees, makes any warranty, express or implied, or assumes any legal liability or responsibility for the accuracy, completeness, or usefulness of any information, apparatus, product, or process disclosed, or represents that its use would not infringe privately owned rights. Reference herein to any specific commercial product, process, or service by trade name, trademark, manufacturer, or otherwise, does not necessarily constitute or imply its endorsement, recommendation, or favoring by the United States Government or any agency thereof. The views and opinions of authors expressed herein do not necessarily state or reflect those of the United States Government or any agency thereof. 


\title{
Simulating High Flux Isotope Reactor Core Thermal-Hydraulics via Interdimensional Model Coupling
}

\author{
A Thesis Presented for the \\ Master of Science \\ Degree \\ The University of Tennessee, Knoxville
}

Adam Ross Travis

May 2014 
To God, first and always: I am nothing other than what He has made me capable of being.

To my parents: If I am able to make good on an ounce of that potential in my life it is because I am the man you raised me to be. 


\section{Acknowledgements}

First and foremost, I would like to express my sincerest thanks and gratitude to Dr. Kivanc Ekici for his encouragement and belief during my tenure as an undergraduate, his unwavering support as my graduate advisor, and the infectious passion which he has always brought to his work. I am similarly indebted to Dr. Jim Freels of Oak Ridge National Laboratory (ORNL) for his constant guidance and mentorship throughout this project. Special thanks as well to Dave Renfro of ORNL for agreeing to fund my work.

All thesis-related work was funded by the Department of Energy (DOE) Office of Science and the Global Threat Reduction Initiative (GTRI) of the National Nuclear Security Administration (NNSA). Further information can be obtained at http://nnsa.energy.gov/

Additional thanks to Dr. Rao Arimilli and Dr. Jay Frankel both for acting as members of my committee and for contributing to the invaluable lessons, both curriculum-related and not, that I have learned at the University of Tennessee.

I wish to express gratitude to those peers who have become close officemates and even closer friends. Thanks to Franklin Curtis for showing me my way around a Linux terminal, to Emily Clark for agreeing with whatever I had to complain about on any given day, and to Kelsey Turley whose unrelenting work ethic kept me in the library on many occasions much later than I would have stayed on my own.

Lastly, I would like to thank my parents Mike and Leigh and my siblings Chloe and Henry for their steadfast support and loving encouragement in every endeavor I set myself to. 


\begin{abstract}
A coupled interdimensional model is presented for the simulation of the thermal-hydraulic characteristics of the High Flux Isotope Reactor core at Oak Ridge National Laboratory. The model consists of two domains - a solid involute fuel plate and the surrounding liquid coolant channel. The fuel plate is modeled explicitly in three-dimensions. The coolant channel is approximated as a twodimensional slice oriented perpendicular to the fuel plate's surface. The two dimensionally-inconsistent domains are linked to one another via interdimensional model coupling mechanisms. The coupled model is presented as a simplified alternative to a fully explicit, fully three-dimensional model. Involute geometries were constructed in SolidWorks. Derivations of the involute construction equations are presented. Geometries were then imported into COMSOL Multiphysics for simulation and modeling. Both models are described in detail so as to highlight their respective attributes - in the 3D model, the pursuit of an accurate, reliable, and complete solution; in the coupled model, the intent to simplify the modeling domain as much as possible without affecting significant alterations to the solution. The coupled model was created with the goal of permitting larger portions of the reactor core to be modeled at once without a significant sacrifice to solution integrity. As such, particular care is given to validating incorporated model simplifications. To the greatest extent possible, the decrease in solution time as well as computational cost are quantified versus the effects such gains have on the solution quality. A variant of the coupled model which sufficiently balances these three solution characteristics is presented alongside the more comprehensive 3D model for comparison and validation.
\end{abstract}




\section{Table of Contents}

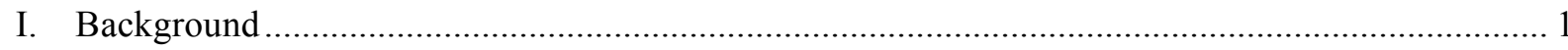

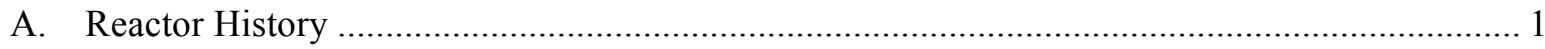

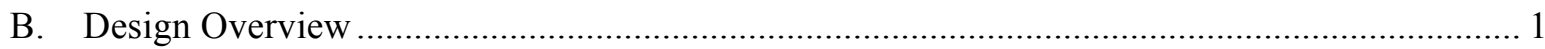

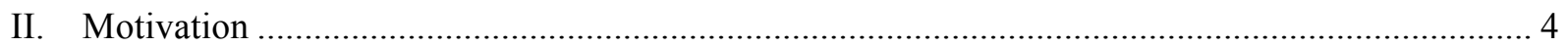

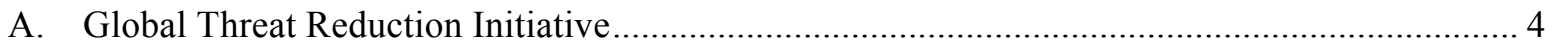

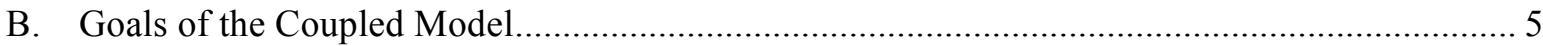

C. Previous Implementations of Interdimensional Coupling ................................................... 6

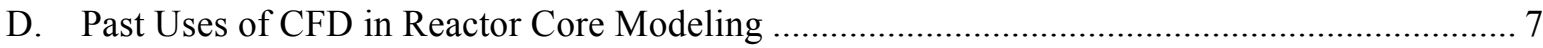

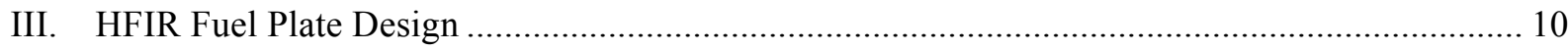

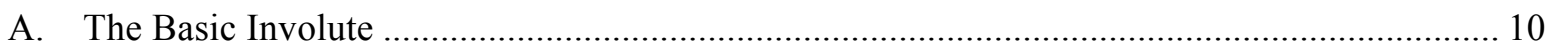

B. Select Alterations to the Basic Involute: ................................................................................. 16

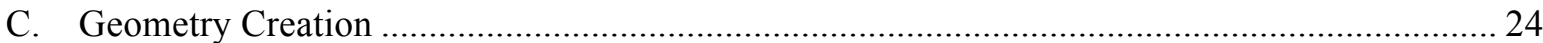

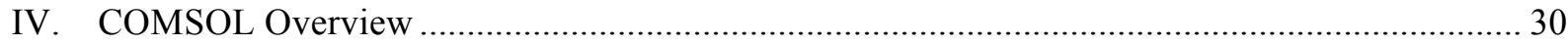

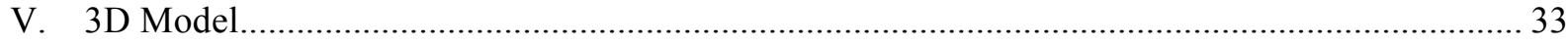

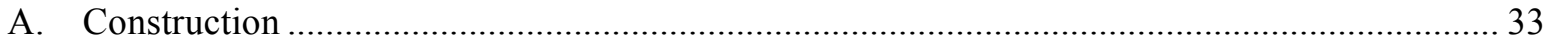

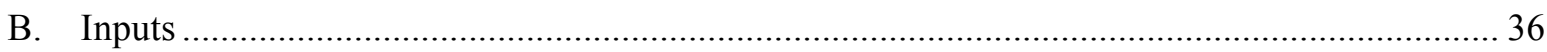

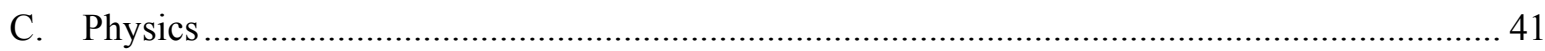

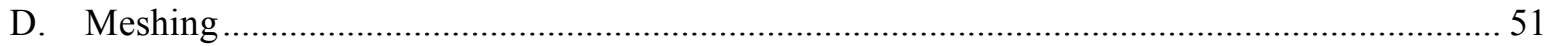

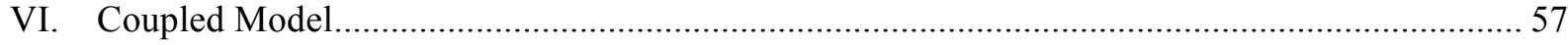

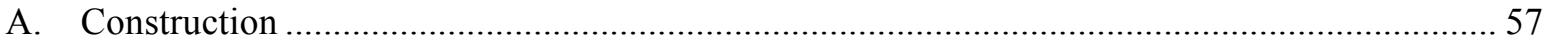

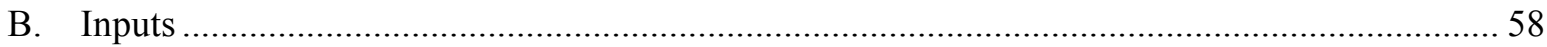

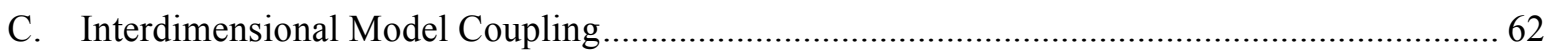

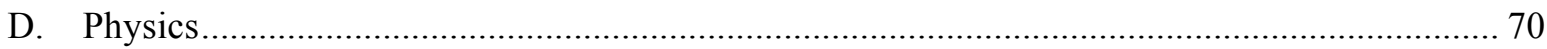

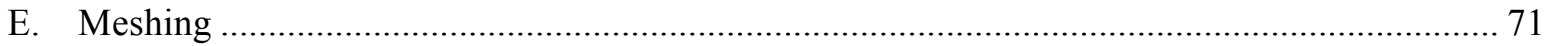

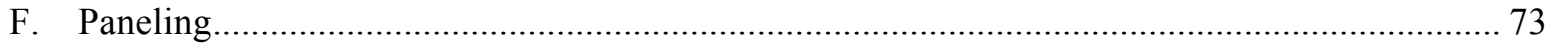




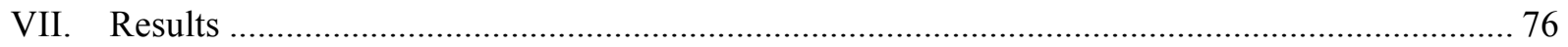

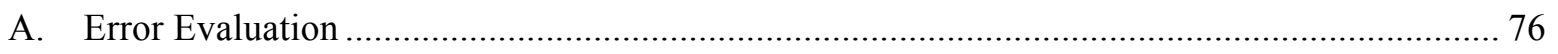

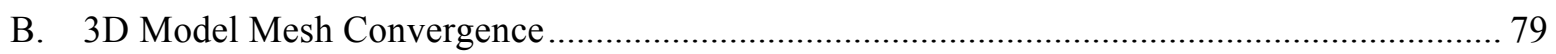

C. Coupled Model Mesh Convergence …........................................................................................ 81

D. Validation of Simplifying Coupled Model Assumptions........................................................ 91

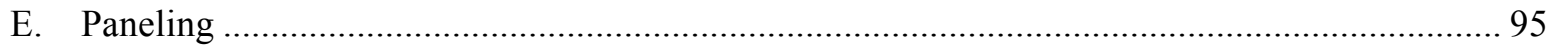

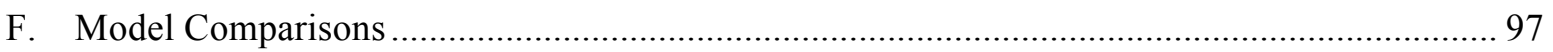

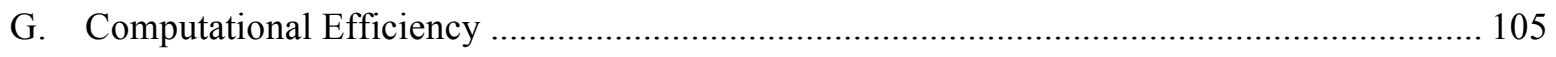

H. Justification of Selected Residual Tolerance ..................................................................... 106

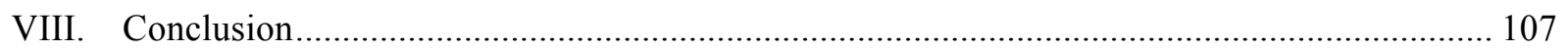

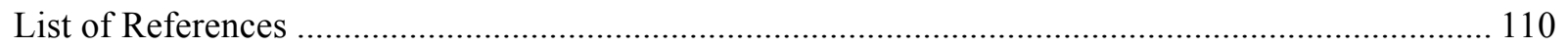

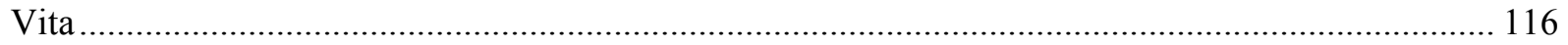




\section{List of Tables}

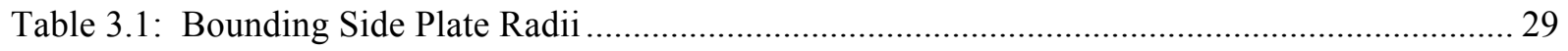

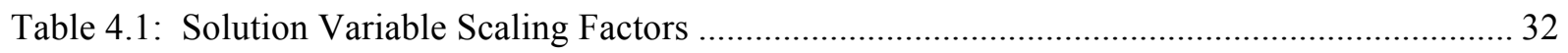

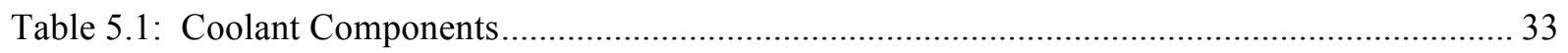

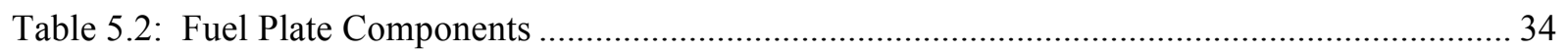

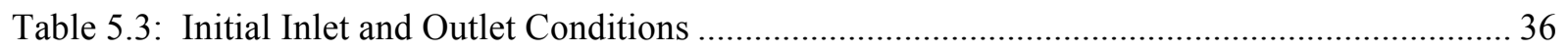

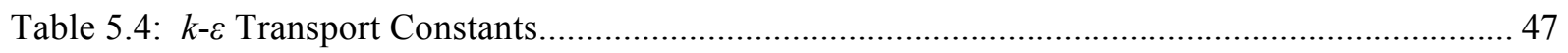

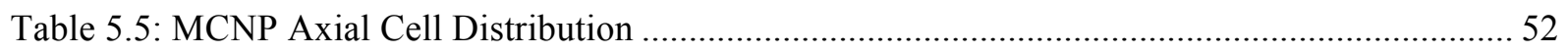

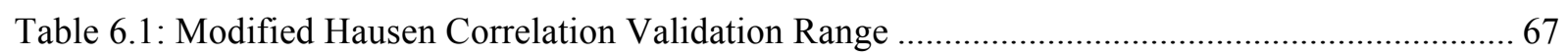

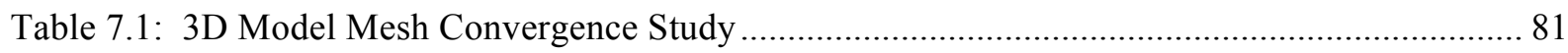

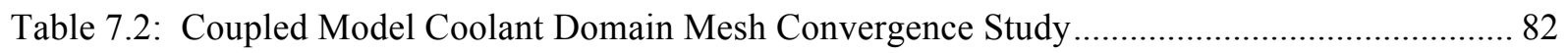

Table 7.3: Coupled Model Fuel Plate Domain Mesh Convergence Study ......................................... 84

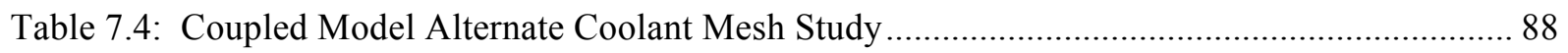

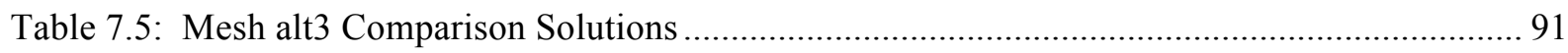

Table 7.6: Propagation of Coolant Smoothing Effect into Fuel Plate.............................................. 105

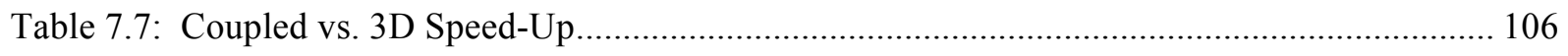

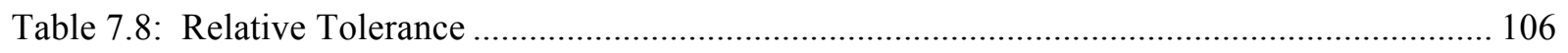




\section{List of Figures}

Fig. 1.1. Top-Down Reactor Vessel Schematic .......................................................................... 2

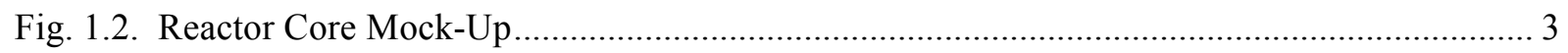

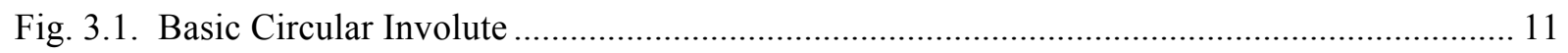

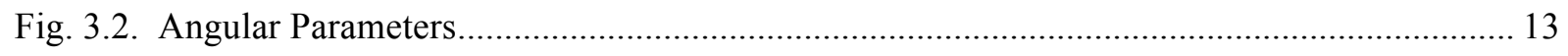

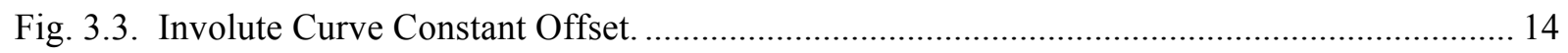

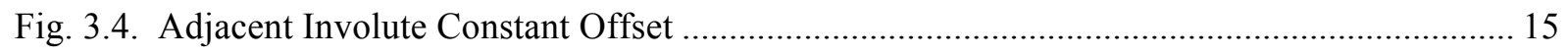

Fig. 3.5. HFIR Core Inner Element Fuel Plate Configuration ..................................................... 16

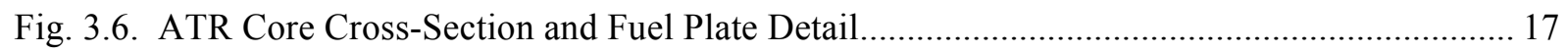

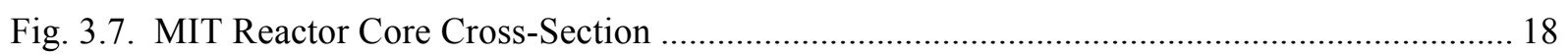

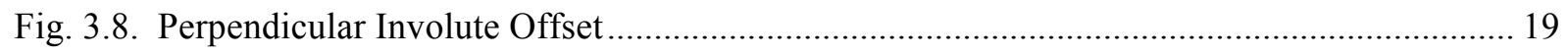

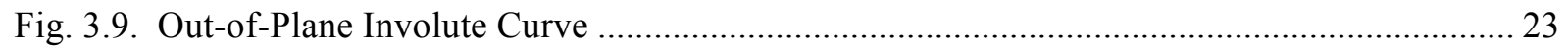

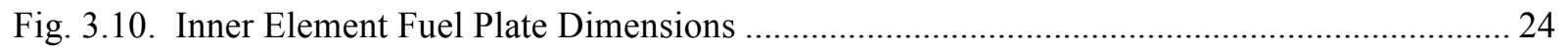

Fig. 3.11. Linearized HEU Fuel Thickness Profile ..................................................................... 25

Fig. 3.12. Linearized LEU Fuel Thickness Profile …............................................................... 26

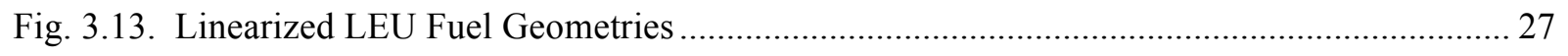

Fig. 3.14. Coolant Channel and Side Plate Periodicity ................................................................ 28

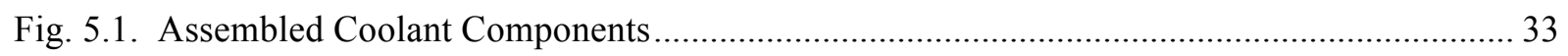

Fig. 5.2. COMSOL Assemblage Side Profile Diagram ............................................................... 34

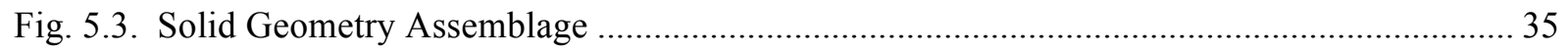

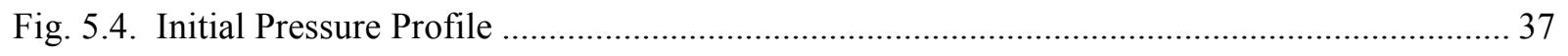

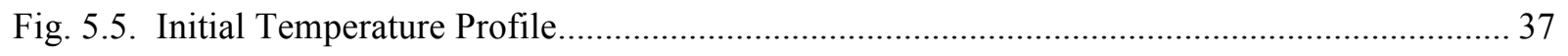

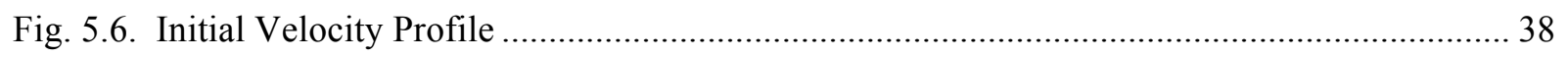

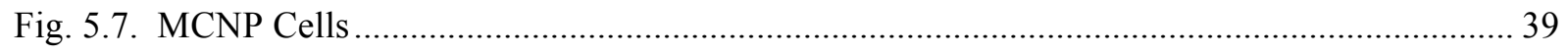




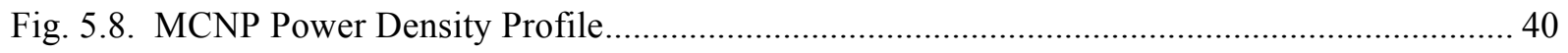

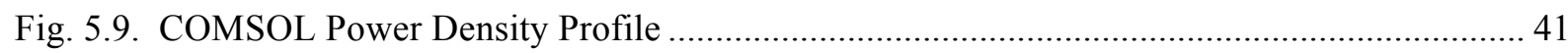

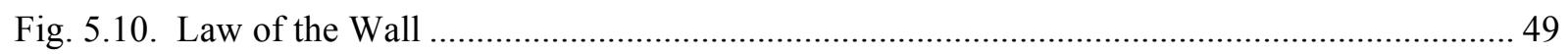

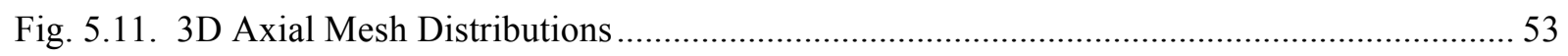

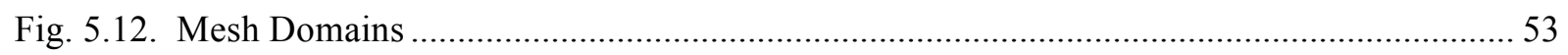

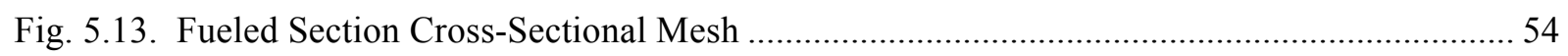

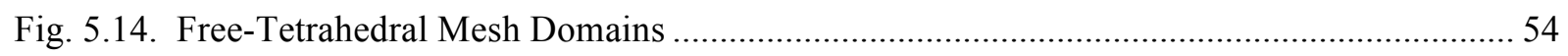

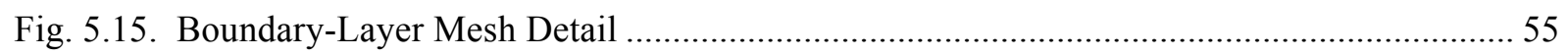

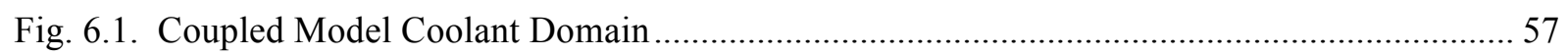

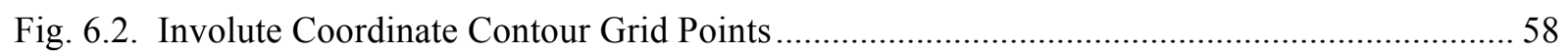

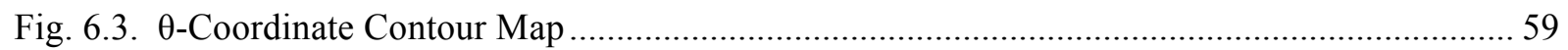

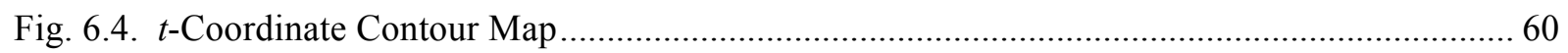

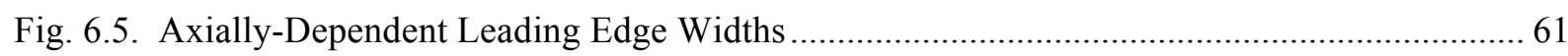

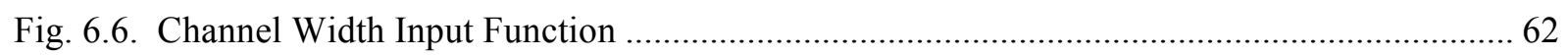

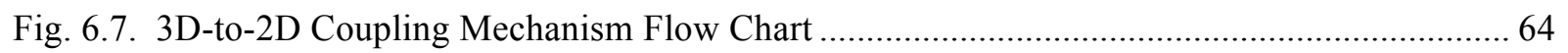

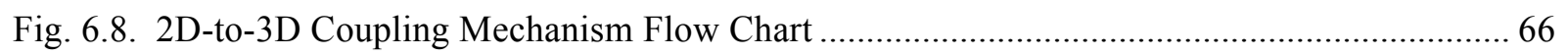

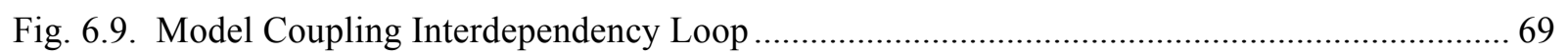

Fig. 6.10. Coupled Model Solution Process Flow Chart................................................................ 70

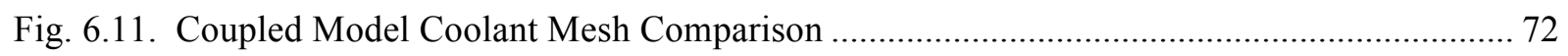

Fig. 6.12. Coupled Model Converted Boundary Layer Detail...................................................... 73

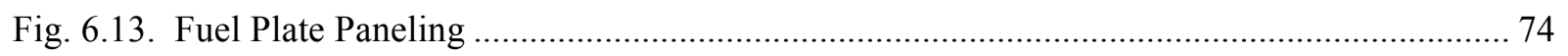

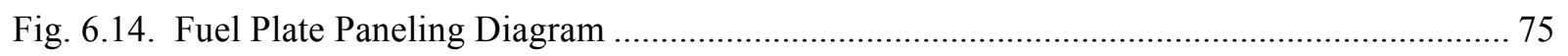

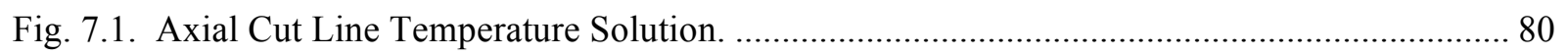

Fig. 7.2. Coupled Model Coolant Mesh Convergence—Coolant Channel Enthalpy ......................... 82

Fig. 7.3. Coupled Model Coolant Mesh Convergence_Logarithmic Coolant Error ......................... 83

Fig. 7.4. Coupled Model Coolant Mesh Convergence-Max Fuel Meat Temperature........................ 83 
Fig. 7.5. Coupled Model Fuel Plate Mesh Convergence-Fuel Plate Error.

Fig. 7.6. Coupled Model Fuel Plate Mesh Convergence-Global Error... 85

Fig. 7.7. Coupled Model Fuel Plate Mesh Convergence-Max Fuel Meat Temperature ... 86

Fig. 7.8. Coupled Model Fuel Plate Mesh p1 87

Fig. 7.9. Coupled Model Fuel Plate Mesh Convergence-Max Fuel Meat T Error. 87

Fig. 7.10. Coupled Model Alternate Coolant Mesh Study-Global Error 89

Fig. 7.11. Coupled Model Alternate Coolant Mesh Study-Max Fuel Meat T..... 89

Fig. 7.12. Mesh alt3 Distribution Comparison. 91

Fig. 7.13. 3D Model Inner Side Plate Inward Heat Flux 92

Fig. 7.14. Coupled Model Hydrodynamic Entrance Region Velocity Profiles 93

Fig. 7.15. Coupled Model Entrance Region Reynolds Number Profile. 94

Fig. 7.16. Coupled Model Thermal Entrance Region Temperature Profiles 94

Fig. 7.17. Conceptual Paneling Model Diagram .95

Fig. 7.18. Conceptual Paneling Model Bulk Temperature Projections 96

Fig. 7.19. Conceptual Paneling Model Surface and Midplane Temperature Profiles. 96

Fig. 7.20. Coupled Paneled Model Concave Fuel Plate Surface Temperature Profile 97

Fig. 7.21. Fuel Meat Concave Surface Temperature Profile Comparison ......................................... 98

Fig. 7.22. Fuel Meat Concave Surface Axial 1D Temperature Profiles ............................................ 98

Fig. 7.23. Fuel Meat Concave Surface Spanwise 1D Temperature Profiles....................................... 99

Fig. 7.24. Cross-Sectional Cut-Out Temperature Profile Comparison …….................................... 100

Fig. 7.25. Cross-Sectional Cut-Out Widthwise Line 1 1D Temperature Profiles............................. 101

Fig. 7.26. Cross-Sectional Cut-Out Widthwise Line 2 1D Temperature Profiles.............................. 101

Fig. 7.27. Coolant Channel Leading Edge Total Velocity Contour Comparison ............................. 103

Fig. 7.28. Coolant Channel Trailing Edge Total Velocity Contour Comparison ............................ 104

Fig. 7.29. Coupled Model Non-symmetric Trailing Edge Pressure Profile..................................... 105 


\section{Nomenclature}

\begin{tabular}{|c|c|c|c|c|c|}
\hline$A$ & involute base circle arc length & (m) & $R$ & Radius & (m) \\
\hline$A_{p}$ & fuel plate surface area & $\left(\mathrm{m}^{2}\right)$ & $r_{c}$ & leading edge radius & (m) \\
\hline$B$ & Spalding coefficient & & $r_{b}$ & involute base circle radius & (m) \\
\hline$c_{p}$ & specific heat at constant pressure & $(\mathrm{J} / \mathrm{kg}-\mathrm{K})$ & $\vec{r}_{i n v}$ & instantaneous involute radial vector & (m) \\
\hline$C_{p}$ & heat capacity at constant pressure & $(\mathrm{J} / \mathrm{K})$ & $\mathrm{R}$ & residual & \\
\hline$C_{1}$ & power profile correction factor & & $\mathrm{R}^{2}$ & coefficient of determination & \\
\hline$C_{\mu}$ & eddy viscosity constant & & $\operatorname{Re}$ & Reynolds number & \\
\hline$C_{\varepsilon 1,2}$ & dissipation constants & & $s$ & involute arc length coordinate & (m) \\
\hline$\dot{C}$ & heat capacity flow rate & $(\mathrm{W} / \mathrm{K})$ & $t$ & involute thickness coordinate & (m) \\
\hline$D_{h}$ & hydraulic diameter & (m) & $T$ & temperature & $(\mathrm{K})$ \\
\hline$E$ & Error & & $T_{b}$ & bulk temperature & $(\mathrm{K})$ \\
\hline$\% e$ & percent error & $(\%)$ & $T_{f}$ & film temperature & $(\mathrm{K})$ \\
\hline$E^{\prime}$ & normalized energy flow rate & & $T_{\text {surf }}$ & surface temperature & $(\mathrm{K})$ \\
\hline$\dot{E}$ & energy flow rate (power) & (W) & $T_{w}$ & wall temperature & $(\mathrm{K})$ \\
\hline$f_{I F E}$ & from inner fuel plates & & $T^{+}$ & dimensionless temperature & \\
\hline$H$ & heat transfer coefficient & $\left(\mathrm{W} / \mathrm{m}^{2}-\mathrm{K}\right)$ & $u$ & velocity & $(\mathrm{m} / \mathrm{s})$ \\
\hline$H^{\prime}$ & normalized enthalpy flow rate & & $u_{\text {tan }}$ & velocity tangent to wall & $(\mathrm{m} / \mathrm{s})$ \\
\hline$\dot{H}$ & total enthalpy flow rate & (W) & $u_{\tau}$ & shear velocity & $(\mathrm{m} / \mathrm{s})$ \\
\hline$I$ & total number of MCNP cells & & $u$ & velocity vector & $(\mathrm{m} / \mathrm{s})$ \\
\hline K & specific turbulent kinetic energy & $(\mathrm{J} / \mathrm{kg})$ & $u^{\prime \prime}$ & fluctuating velocity vector & $(\mathrm{m} / \mathrm{s})$ \\
\hline$L_{h}$ & heated length & (m) & $\begin{array}{l}\widetilde{\boldsymbol{u}} \\
V\end{array}$ & $\begin{array}{l}\text { Favre-averaged velocity vector } \\
\text { volume }\end{array}$ & $\begin{array}{l}(\mathrm{m} / \mathrm{s}) \\
\left(\mathrm{m}^{3}\right)\end{array}$ \\
\hline$\hat{n}$ & boundary normal vector & & $V_{\text {fuel }}$ & volume of fuel in an MCNP cell & $\left(\mathrm{m}^{3}\right)$ \\
\hline $\begin{array}{l}n_{I F E} \\
\mathrm{~N}\end{array}$ & $\begin{array}{l}\text { total number of inner fuel plates } \\
\text { Navier-Stokes operator }\end{array}$ & & $V_{\text {ratio }}$ & $\begin{array}{l}\text { MCNP fuel to total cell volume } \\
\text { ratio }\end{array}$ & \\
\hline $\mathrm{Nu}$ & Nusselt number & & $w_{c}$ & & 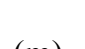 \\
\hline & & & & & (mi) \\
\hline $\mathrm{Nu}_{b m}$ & original mean Hausen correlation & & $w_{r}$ & leading edge width & (m) \\
\hline $\mathrm{Nu}_{b z}$ & modified local Hausen correlation & & $w_{v}$ & void width & (m) \\
\hline$P$ & Pressure & $(\mathrm{Pa})$ & $x, y$ & Cartesian coordinates $1 \& 2$ & (m) \\
\hline$p^{\prime \prime}$ & fluctuating pressure & $(\mathrm{Pa})$ & $y_{c}$ & perpendicular wall coordinate & $(\mathrm{m})$ \\
\hline $\begin{array}{l}\widetilde{p} \\
P\end{array}$ & $\begin{array}{l}\text { Favre-averaged pressure } \\
\text { power }\end{array}$ & $\begin{array}{l}(\mathrm{Pa}) \\
(\mathrm{W})\end{array}$ & $y_{c}^{+}$ & dimensionless wall coordinate & \\
\hline$P^{\prime \prime \prime}$ & power density & $\left(\mathrm{W} / \mathrm{m}^{3}\right)$ & $z$ & axial coordinate (Cartesian 3 ) & (m) \\
\hline $\operatorname{Pr}$ & Prandtl number & & $z_{c}$ & axial coordinate $2 \mathrm{D}$ domain & (m) \\
\hline $\operatorname{Pr}_{T}$ & turbulent Prandtl number & & $z_{p}$ & axial coordinate 3D domain & $(\mathrm{m})$ \\
\hline $\operatorname{Pr}_{T_{\infty}}$ & turbulent Prandtl number at infinity & & & & \\
\hline$q^{\prime \prime}$ & heat flux vector & $\left(\mathrm{W} / \mathrm{m}^{2}\right)$ & $\alpha$ & involute arc angular coordinate & (rad) \\
\hline$q_{n}^{\prime \prime}$ & boundary normal heat flux & $\left(\mathrm{W} / \mathrm{m}^{2}\right)$ & $\begin{array}{l}\alpha_{o} \\
\delta_{w}\end{array}$ & $\begin{array}{l}\text { initial involute arc angular coord } \\
\text { wall-offset distance }\end{array}$ & $(\mathrm{rad})$ \\
\hline$Q$ & volumetric heat generation & $\left(\mathrm{W} / \mathrm{m}^{3}\right)$ & & & \\
\hline
\end{tabular}




\begin{tabular}{|c|c|c|c|c|c|}
\hline$\delta_{w}^{+}$ & dimensionless wall-offset & $(\mathrm{m})$ & $\tau_{R}$ & $\begin{array}{l}\text { specific Fave-averaged stress } \\
\text { tensor }\end{array}$ & $\left(\mathrm{m}^{2} / \mathrm{s}^{2}\right)$ \\
\hline$\Delta \dot{H}_{c}$ & $\begin{array}{l}\text { change in coolant enthalpy flow } \\
\text { rate }\end{array}$ & $(\mathrm{W})$ & $\tau_{S}$ & viscous stress tensor & $\left(\mathrm{m}^{2} / \mathrm{s}^{2}\right)$ \\
\hline E & dissipation rate of turbulent energy & $(\mathrm{J} / \mathrm{kg}-\mathrm{s})$ & $\phi$ & dummy variable & \\
\hline$\eta_{T, d t, \phi}$ & intermediate coupling variables & & $\phi_{n}$ & dummy variable finite element & \\
\hline$\Theta$ & involute base circle angular coord & $(\mathrm{rad})$ & & & \\
\hline$\theta_{o}$ & initial base circle angular coord & $(\mathrm{rad})$ & $\phi^{\prime \prime}$ & fluctuating dummy variable & \\
\hline$K$ & von Kármán constant & & $\widetilde{\phi}$ & Favre-averaged dummy variable & \\
\hline$\Lambda$ & thermal conductivity & $(\mathrm{W} / \mathrm{m}-\mathrm{K})$ & $\Psi$ & finite element basis function & \\
\hline$M$ & dynamic viscosity & $(\mathrm{kg} / \mathrm{m}-\mathrm{s})$ & & & \\
\hline$\mu_{T}$ & dynamic eddy viscosity & $(\mathrm{kg} / \mathrm{m}-\mathrm{s})$ & $\Im$ & linear operator & \\
\hline$\mu_{w}$ & wall dynamic viscosity & $(\mathrm{kg} / \mathrm{m}-\mathrm{s})$ & & & \\
\hline$N$ & kinematic viscosity & $\left(\mathrm{m}^{2} / \mathrm{s}\right)$ & & & \\
\hline$\Pi$ & Nusselt number stand-in variable & & & & \\
\hline$P$ & Density & $\left(\mathrm{kg} / \mathrm{m}^{3}\right)$ & & & \\
\hline $\mathrm{P}_{k}$ & turbulence production term & $\left(\mathrm{m}^{2} / \mathrm{s}^{2}\right)$ & & & \\
\hline$\sigma_{k, \varepsilon}$ & $k-\varepsilon$ closure coefficients & & & & \\
\hline
\end{tabular}




\section{Background}

\section{A. Reactor History}

The High Flux Isotope Reactor (HFIR) at Oak Ridge National Laboratory (ORNL) was constructed as a result of a review of the United States' transuranium isotope production capabilities by the now-defunct Atomic Energy Commission in 1958. ${ }^{1}$ To this day, HFIR maintains the distinction of producing the highest research reactor-based neutron flux, approximately $4 \times 10^{15}$ neutrons $/ \mathrm{cm}^{2} / \mathrm{s}$, in the United States. ${ }^{2}$ In the decades since its activation, HFIR's capabilities have expanded and evolved with the country's needs. Today the reactor is used for material irradiation, neutron activation analysis, and neutron scattering studies in addition to the production of transuranium isotopes. Most notably, HFIR is the sole producer of Californium-252 $\left(\mathrm{Cf}^{252}\right)$ in the western world. $\mathrm{Cf}^{252}$ is used in pollution and explosives detectors as well as cancer therapy. ${ }^{3}$ HFIR is slated to begin production of plutonium-238 $\left(\mathrm{Pu}^{238}\right)$, the fuel source in Radioisotope Thermoelectric Generators (RTGs) employed by NASA aboard interplanetary spacecraft. Prior to HFIR's on-going efforts to become a stable provider of $\mathrm{Pu}^{238}$, the United States had been without a production source since 1988 forcing the country to rely on Russia to sell off portions of their own stores of the isotope. ${ }^{4}$ The reactor has even been used by the candy manufacturer Mars, Inc. to perfect its pretzel M\&M formula. The reactor's neutron flux was used to produce detailed images of the candy pieces in order to analyze the bleed-through of water for the various colored shells. ${ }^{5}$ These are but a select few of the numerous applications of HFIR's neutron flux.

\section{B. Design Overview}

HFIR was designed with an intended power output of $100 \mathrm{MW}$. But accelerated reactor vessel embrittlement due to neutron irradiation was identified in 1986, prompting the reactor's power output to be dropped to $85 \mathrm{MW}$ ever since. ${ }^{6}$ Subsequent reactor vessel analysis has shown this level of power output to be sustainable in this regard. Current projections predict that HFIR will continue to operate well into 2040 and beyond. The reactor itself is water-cooled, having coolant pumped in vertically through two concentric circular annuli at a flow rate of approximately $1.0 \mathrm{~m}^{3} / \mathrm{s}$. The system is designed to operate at an inlet pressure of $3.3 \mathrm{MPa}$ with a velocity of $7.8 \mathrm{~m} / \mathrm{s}$ and a temperature of $322 \mathrm{~K}$. With the current high-enriched uranium (HEU) fuel configuration, the coolant undergoes a $20 \mathrm{~K}$ temperature increase from 
inlet to outlet with a pressure drop of $0.76 \mathrm{MPa}^{7}$ A top-down schematic of the reactor core and adjacent experimental setups is shown in Fig. 1.1.

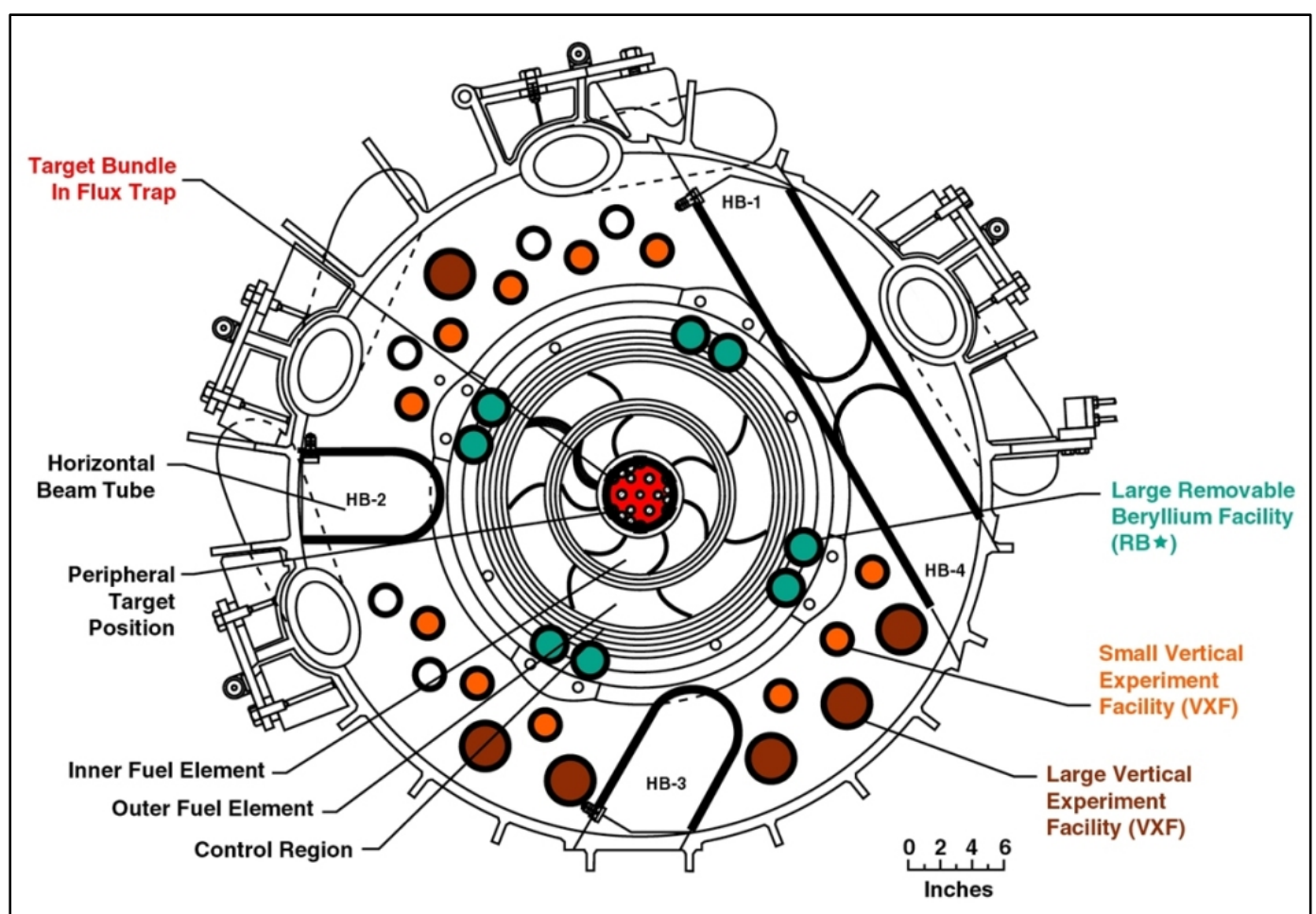

Fig. 1.1. Top-Down Reactor Vessel Schematic. ${ }^{8}$ The direction of flow through the core is into the page. Coolant flows through the two circular annuli between the target flux trap and the removable control plates. The beam tubes are the primary experimental setups used in the reactor.

The reactor core is divided into two radial elements, inner and outer, with 171 and 369 fuel plates, respectively. A mock-up of the reactor core's fuel element section is shown in Fig. 1.2. Each fuel element is held between two concentric cylindrical side plates. The side plates and the outer cladding of the fuel elements are made of aluminum-6061. ${ }^{9}$ 


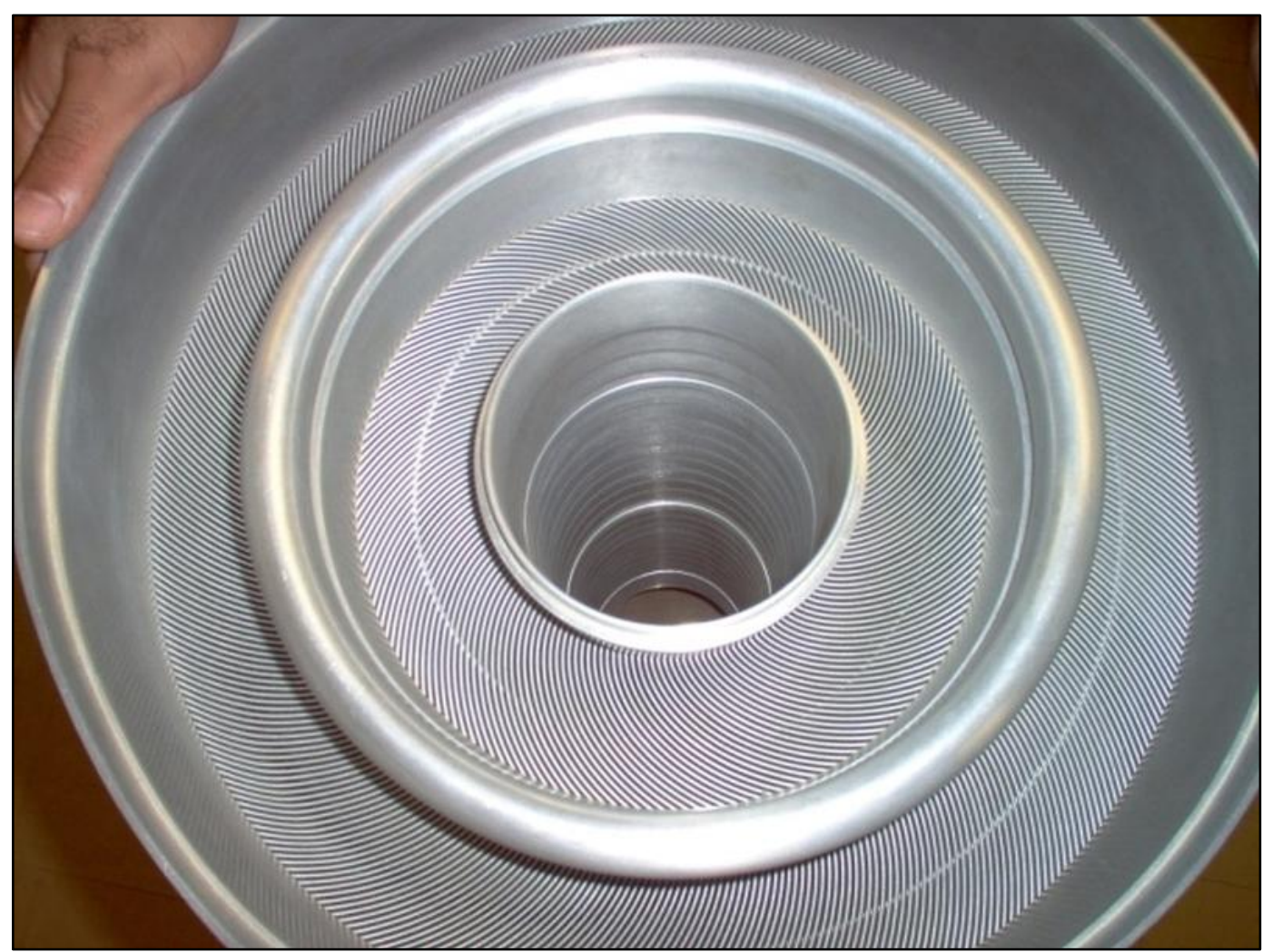

Fig. 1.2. Reactor Core Mock-Up. ${ }^{8}$ Inner and outer elements are both shown. The axial hole through the center of the core is occupied by the target flux trap during use. The direction of flow is from top to bottom. This mock-up has an added element known as a "comb" that is not present in the actual HFIR core design. The comb can be seen bisecting the concentric mid-plane of both elements approximately $3 \mathrm{~mm}$ below the leading edge. 


\section{Motivation}

\section{A. Global Threat Reduction Initiative}

As mentioned in Section I, HFIR was constructed to utilize high-enriched uranium (HEU) fuel. Uranium fuel's grading is based on its percentage of uranium-235 $\left(\mathrm{U}^{235}\right)$ by weight. The remainder of the fuel is composed of $\mathrm{U}^{238}$, a more abundant uranium isotope found commonly in nature. HEU fuel is characterized by a $\mathrm{U}^{235}$ concentration of at least $20 \%$ or greater. Anything below this level is considered low-enriched uranium (LEU). As a basis for comparison, naturally occurring un-enriched uranium has a $\mathrm{U}^{235}$ concentration of only $0.711 \%$. The HEU fuel currently utilized by HFIR is $93.1 \% \mathrm{U}^{235}$ by weight. ${ }^{10}$ This high concentration of $\mathrm{U}^{235}$ makes HFIR's current fuel composition an efficient and desirable fuel source. With a $\mathrm{U}^{235}$ concentration greater than $90 \%$, however, it also puts it in the classification of weapons-grade uranium. ${ }^{11}$

The conversion of nuclear reactor sites worldwide from HEU to LEU fuel sources has been a goal of the Department of Energy (DOE) for over three decades. In order to facilitate the widespread conversion of nuclear reactors, the DOE created the Reduced Enrichment for Research and Test Reactors (RERTR) program in 1978. Over 40 reactors have successfully made the conversion since the program's inception. ${ }^{12}$

A handful of the country's high-flux reactors remain unconverted at present, however, due primarily to their exceptional output requirements and design complexity. These reactors include the Advanced Test Reactor (ATR) at Idaho National Laboratory, the National Institute of Standards and Technology (NIST) research reactor, the Missouri University Research Reactor (MURR), the Massachusetts Institute of Technology Research (MITR-II) reactor, and HFIR at ORNL. ${ }^{13}$

Increased interest has arisen in the past decade in converting these five remaining holdouts to LEU fuel sources with the creation of the Global Threat Reduction Initiative (GTRI) by the National Nuclear Security Administration (NNSA) in 2004. The GTRI has taken a more aggressive security-oriented approach to removing HEU fuel capable of being formed into a weapon of mass destruction (WMD), referred to as "WMD-usable" sources, from the world in order to prevent theft and misuse. The initiative's mission is to convert or shutdown all HEU-powered reactors, remove all excess HEU materials, and protect any remaining sources from theft. To that aim it claims to have converted or 
confirmed the shutdown of $88 \mathrm{HEU}$ research reactors, removed over 2,500 kilograms of HEU-the equivalent of over 140 nuclear bombs - and secured more than 1,500 radiological sites across the globe. ${ }^{14}$

This global effort from the NNSA has spurred renewed national interest in converting the five remaining research reactors as a means of demonstrating commitment to its international partners. The same issues regarding exacting output criteria and complicated design geometries still apply, however. A project with the goal of researching the feasibility of converting HFIR to an LEU fuel source was started in 2004 and is currently scheduled to conclude in 2030.

\section{B. Goals of the Coupled Model}

The primary purpose of this thesis will be to present a novel computer model for the study of the thermal-hydraulics of the HFIR core. This model will be referred to herein as the coupled model. The intent of the coupled model is two-fold: To model, explicitly and in sufficient detail, the flow of energy in the form of heat from the fissile fuel plates in the reactor core to the surrounding coolant in as computationally-inexpensive a manner as possible. The first of these aims is due to the close relationship between various reactor component temperatures and the integrity of the reactor. An abnormality in the former begets a deviation from the latter. Understanding the heat transfer internal to the reactor core, both through the fuel plate itself and from the fuel plate into the coolant, is essential to predicting the reactor's performance.

Three primary variables of interest are the maximum temperatures in the fuel meat, the fuel plate, and the coolant channel. The first two must remain below the melting points of their respective materials while the third must remain below its incipient boiling point at which nucleate boiling gives rise to twophase flow. The maximum coolant temperature can be extrapolated as a function of the first two through wall superheat relations ${ }^{15}$. The maximum temperatures in the solid domains - and, to a lesser extent, the temperature profiles in each — are therefore the primary concerns of the coupled model.

The coupled model utilizes this fact in realizing its second goal of simplifying the model as much as possible. In order to cut down on the degrees of freedom (DOF) in the model, the coolant domain is represented via a simplified 2D slice. The fuel plate and fuel meat are represented explicitly in 3D. The pairing of the two dimensionally inconsistent domains, solid and coolant, is achieved through interdimensional model coupling. In this configuration, with the focus of the model being on the solid domain, the coolant domain essentially serves as an adaptive boundary condition. 
A fully three-dimensional model, referred to herein as the 3D model, will be presented alongside the coupled model as a means of comparison. Where the coupled model seeks to simplify the computational domain as much as possible, the 3D model seeks to simulate the same domain with as much detail and accuracy as is reasonably possible given available computational resources. This serves to provide a metric for the degree of inaccuracy and loss of fidelity introduced in the simplifications of the coupled model.

\section{Previous Implementations of Interdimensional Coupling}

Blanco et al. ${ }^{16}$ make a case for the use of interdimensional model coupling in the capacity of an adaptive boundary condition versus the implementation of a static Dirichlet or Neumann boundary condition, which impose a certain degree of rigidity on the evolving solution's ability to adapt to changing conditions. They coin this adaptive feedback across the interdimensional coupling the "systemic response" of the entire coupled domain.

Interdimensional model coupling has appeared in many applications across many fields ranging from thermal circuitry analysis to structural mechanics and computational fluid dynamics. In the vein of producing a systemic response, the method has been applied in particular to systems modeling in which a complex, or at least multi-component, system is modeled with varying degrees of detail throughout. For any given component in a system, it at once influences the rest of the system even as the system imparts an influence back upon it. This breeds a looped dependency across coupling interfaces in which boundary conditions must be formatted and shared. ${ }^{17}$

There has been a particular interest in coupling fluid domains in an effort to reduce the computational costs associated with solving the Navier-Stokes equations in three-dimensions in large solution domains. ${ }^{18}$ Systems in which the flow impedance of a single component-ranging from a valve ${ }^{19}$ to a burst dam $^{20}$ — requires detailed modeling have been attached to pipe flow models as a means of enforcing reactive boundary conditions. This has been applied in the automotive industry using the full 3D N-S equations to model the cavitation flow around fuel injection nozzles while utilizing 1D upstream systems to provide accurate inlet conditions. ${ }^{21}$

Interdimensional model coupling has also found prevalence in the study of large flow environments such as rivers, lakes, and ocean currents where 2D surface flow mingles with 3D sub-surface flow effects. $^{22,23,24,25}$ Similar work has been done with a focus on locally selected regions of detailed 
resolution amidst a broader solution domain, especially with regards to flows with impeding structures and blockages ${ }^{26,27}$ and flows over uneven surfaces. ${ }^{28,29,30}$

Miglio et $a .^{31}$ lump model coupling efforts into two categories: dimensionally homogeneousphysically heterogeneous couplings and dimensionally heterogeneous-physically homogeneous couplings. The former is concerned with the coupling of dissimilar physics models, such as fluid flow and solid heat conduction, in dimensionally consistent domains. The latter category constitutes interdimensional model couplings in which the same physics model is considered across dimensionally inconsistent domains. The bulk of examples cited above fall into this latter category.

The groupings are incomplete, however, due to their mutually exclusive nature. They assume that if one form of coupling is present then the other must not be. In order to rank the coupled model according to this rubric a third category must be introduced - that of the dimensionally heterogeneous-physically heterogeneous coupling. This constitutes a coupling occurring across dissimilar physics models and dimensionally inconsistent domains.

Not only does this provide a convenient interface across which to couple, but it also breaks the model down into two discrete domains with only minimal interaction between them. This results in a much smaller model which is the focus of this work. Note that this model requires neither the computational resources nor the solution time of a fully three-dimensional model while still returning comparable results. This makes it an efficient and reasonably accurate alternative to the full-3D approach.

\section{Past Uses of CFD in Reactor Core Modeling}

Many nuclear reactors in operation today were designed well before the advent of computational fluid dynamics (CFD) as a mainstream engineering tool. Even recently constructed reactors are largely derivatives of past models, built on relatively well understood principles of nuclear engineering and design. While commercial and open-source codes have found uses in this process, they tend to be either systems based, relegating the reactor core itself to a zero-dimensional node in a loop, or are heavily reliant upon experimentally derived correlations, limiting any such code's use to a narrow window of optimal operating conditions. While experimentation is invaluable as a means to providing validation for simulations, the ability to model a given reactor core condition with relative confidence should not be hamstrung due to a lack of experimental data from that exact condition. Explicitly modeling the reactor core using CFD not only expands the applicability of such models to various and dissimilar operating conditions, it also allows for preemptive simulations and accident analysis to be performed. 
An early standout in this regard is the work of Anglart et al. ${ }^{32}$ who used a commercial CFD code known as CFDS-FLOW3D to simulate two-phase flow in a boiling water reactor (BWR). In their work, they make note of the difficulties associated with explicitly modeling reactor core thermal-hydraulics, owing primarily to complex internal geometries and numerical difficulties. More contemporary work has been performed by Krepper et $a l^{33}$ who, citing the limiting effects of $1 \mathrm{D}$ core simulations and their inability to account for the effects of internal core geometries, used FLUENT to explicitly model mass and heat flow in $3 \mathrm{D}$ around such geometries.

Similar work was performed in the modeling of BWRs using FLUENT by Kocar and Sokmen ${ }^{34}$ who built upon the earlier advances of Lahey and Drew ${ }^{35}$ and Končar et al. ${ }^{36}$ in the modeling of two-phase flow. Rohde et al. ${ }^{37}$ used a combination of CFX and FLUENT to model coolant circulation in pressurized water reactors (PWRs). Their focus was on tracking boron used in the coolant to counteract excess reactivity in freshly installed fuel. Using CFD they were able to simulate the mixing which occurred inside the reactor core and were thus able to determine local boron concentrations.

These studies represent isolated cases in which CFD was used to understand flow conditions in preexisting reactors. Only in the most recent reactor design initiatives does one see the use of CFD rise to prominence. The next generation of nuclear reactors has been labelled generation IV. With plans to begin operation before 2030, the design for many generation IV reactors is still in the predominantly theoretical stage. Generation IV reactor designs are all different variations on the concept of a very high temperature reactor (VHTR) ${ }^{38}$ One of these is the Pebble Bed Modular Reactor (PBMR) project in South Africa. CFD has played an increasingly prominent role in the PBMR's design since the project's inception in 1994. Becker and Laurien ${ }^{39}$ used CFX-D to conduct early 3D simulations of the PBMR, focusing their work on the modeling of possible accident-related non-axially symmetric core operating conditions.

Shang $^{40}$ has applied 3D CFD to the research process for another generation IV reactor, the Supercritical Water-Cooled Nuclear Reactor (SCWR). Using STAR-CD he was able to investigate alternative fuel rod geometry configurations, an ability which had been nonexistent prior to his work due to the limitations of 2D models. Johnson, ${ }^{41}$ similarly, used STAR-CCM+ to simulate coolant flow and heat transfer as it exits the reactor vessel of the proposed Next Generation Nuclear Plant (NGNP) design in the US. In their work, Baqué et al., ${ }^{42}$ state future plans to make use of CFD to model the effect of internal core geometry on coolant flow rates in the proposed Sodium-Cooled Fast Reactor (SFR) in France. Schulenberg et $a .^{43}$ mention the critical role that CFD has played in multiple aspects of the 
design of the European High Performance Light Water Reactor (HPLWR). Ferng and Chen ${ }^{44}$ have conducted hypothetical accident analyses of the prototype HTR-10 reactor in China using FLUENT.

Interdimensional model coupling has even found rare use in reactor core modeling. Many of these couplings include a link between a systems-level code such as RELAP5 or Flownex to model the entire reactor loop and a CFD package to explicitly model the reactor core itself. 1D inputs are fed into the reactor core node from the systems-level code which the CFD model then uses as inlet conditions with which to drive internal core flow. Outlet conditions are then homogenized and fed back into the reactor loop.

Jeong et $a l .{ }^{45}$ and Lee et $a l .{ }^{46}$ both created such couplings using RELAP5 to model the reactor loop and COBRA to create a localized 3D hydrodynamic model. Aumiller et al ${ }^{47}$ created a similar coupling using RELAP5 and CFX. Walter et al., ${ }^{48}$ in work related to the design of the PBMR, have coupled Flownex to model the reactor loop and a 2D neutronics/thermal-hydraulics code known as RZKIND to model the core. Perhaps the most elaborate instance of reactor code coupling comes from the work of Venter and Mitchel1 ${ }^{49}$ who used a combination of Flownex and two CFD packages, STAR-CD and FLUENT, to create a multiphysics framework combining elements of neutronics, CFD, shielding, and systems-level modeling. 


\section{HFIR Fuel Plate Design}

\section{A. The Basic Involute}

The HFIR core fuel plate takes the shape of a circular involute curve. The concept of an involute was first proposed by Christiaan Huygens in 1673. Involutes and their opposites, evolutes, have played prominent roles in the development of early pendulum clocks and mechanical gearing tooth designs. ${ }^{50,51}$ A simple means of envisioning the path a circular involute takes is to consider a tetherball wound around a pole. The circular involute is the locus of points traced by the tetherball as it is unwound from the pole. Its position is a function of the increasing length of the tangent line emanating from sequential points along the perimeter of the base circle. By contrast, if the circular involute were considered the original function and the circle was generated by means of the opposite procedure, the circle would be considered its evolute. ${ }^{52}$ A circular involute is shown in Fig. 3.1. The basic circular involute is given by the relation

$$
\vec{r}_{i n v}=r_{b}\{[\cos (\theta)+\theta \sin (\theta)] \hat{j}+[\sin (\theta)-\theta \cos (\theta)] \hat{j}\}, \quad \theta \geq 0
$$

where $r_{b}$ is the radius of the base involute, assumed to be positive, and $\theta$ is measured in radians. ${ }^{53}$ The circular involute's reliance on a base circle is easy to see in this formulation. In comparison with the equation for a circle

$$
\left.\vec{r}_{i n v}=r_{b}\{\cos (\theta)+\sin (\theta)] \hat{j}+[\sin (\theta)-\cos (\theta)] \hat{j}\right\}
$$

it is apparent that the circular involute equation is the same equation merely with a constantly-increasing amplitude attached to the second term in each bracket. For the basic circular involute this constantlyincreasing amplitude is a direct function of the angular position $\theta$. 


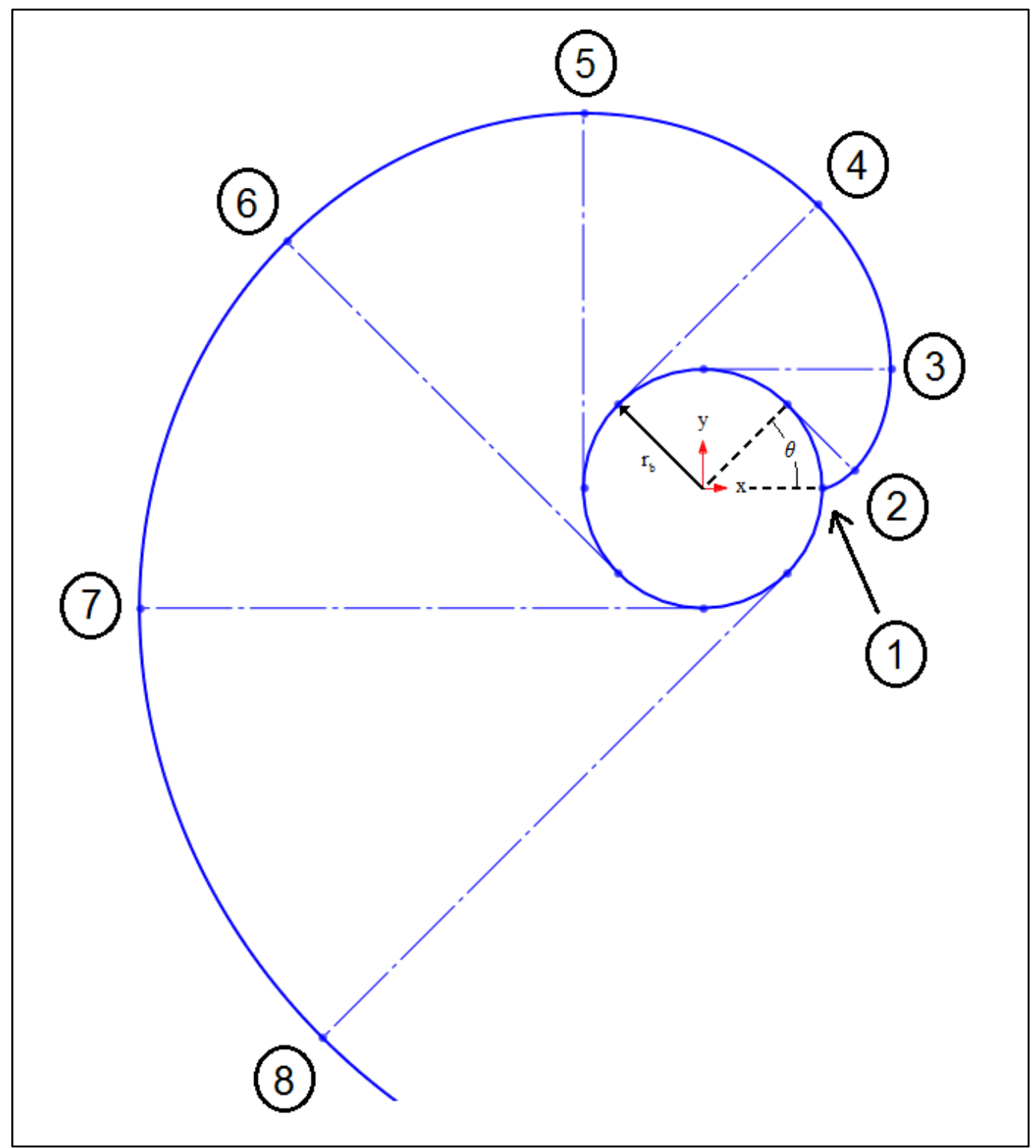

Fig. 3.1. Basic Circular Involute. The involute curve is shown along with its base circle. Tangent guidelines are represented by dashed lines. The numbers indicate the direction of progression of the involute in the direction deemed "positive."

For the purpose of brevity, the basic circular involute will be called the basic involute herein. The fact that it is a circular involute will be implied as this is the only involute curve detailed explicitly. The basic involute in Eqn. (3.1) can be expressed in parametric form such that

$$
x=r_{b}[\cos (\theta)+\theta \sin (\theta)] \quad \theta \geq 0
$$




$$
y=r_{b}[\sin (\theta)-\theta \cos (\theta)] \quad \theta \geq 0 .
$$

While this is considered the basic involute equation, in truth however, this is a simplification of a more generalized form. In this more general form there are two variables, $\theta$ and $\alpha$, where one is a linear function of the other. This generalized form is given by the parametric equation

$$
\begin{gathered}
x=r_{b}[\cos (\theta)+\alpha \sin (\theta)] \quad \alpha \geq 0 \\
y=r_{b}[\sin (\theta)-\alpha \cos (\theta)], \alpha \geq 0 \\
\alpha=\Im(\theta)
\end{gathered}
$$

where the $\mathfrak{I}$ operator denotes that $\alpha$ is a linear function of $\theta$. In this generalized form, the variable $\theta$ specifies the angular position of the tangent line emanating from the perimeter of the base circle. The variable $\alpha$ specifies the angular position along the length of the involute curve itself. Or, in terms of the tetherball analogy, $\theta$ describes the point of contact of the tether with the pole and $\alpha$ describes the corresponding location of the tetherball. An example of this is shown in Fig. 3.2.

Using this generalized form of the parametric equation, an entire array of involute curves can be created by varying $\theta_{\mathrm{o}}$. In fact, the circular involute was chosen for the shape of the fuel plates due to its characteristics under such a circumstance. If carried out far enough a circular involute produces a spiral. The distance between successive spirals measured at any point perpendicular to both is a constant, as shown in Fig. 3.3. ${ }^{54}$

The circular involute is not to be confused with the Archimedean spiral, which has similar characteristics, in this regard. Where the circular involute is the locus of points resulting from the tetherball spiral mentioned above, the Archimedean spiral is the locus of points generated by a point moving away from a fixed center with constant speed along a line rotating around that same fixed center with constant angular velocity. The Archimedean spiral is given by the relation ${ }^{54}$

$$
\begin{gathered}
\vec{r}_{a r c}=[r \cos (\theta)] \hat{j}+[r \sin (\theta)] \hat{j} \\
r=\Im(\theta) .
\end{gathered}
$$




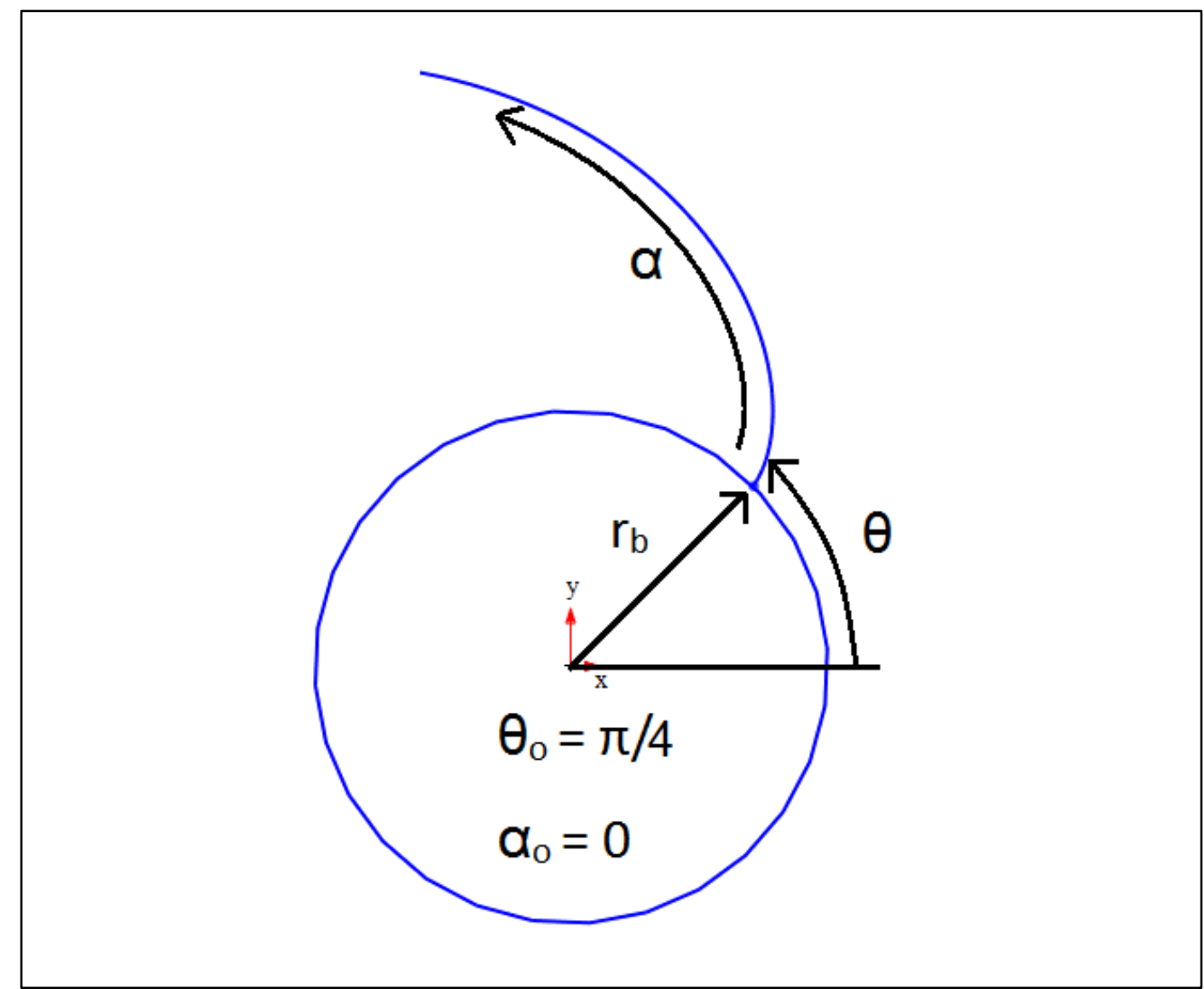

Fig. 3.2. Angular Parameters. In this example $\alpha=\theta-\pi / 4$. Instances in which $\alpha$ does not start at zero are also possible. These will, however, have some angular offset separating them from contact with the base circle.

The two curves are similar looking. And indeed, their characteristics are also very similar. Yet they have one key difference. The distance between successive spirals in an Archimedean spiral is constant only when measured from a ray emanating from the fixed center of rotation. ${ }^{54}$ The Archimedean spiral's constant offset has no relation to orthogonality with each of the spirals like the basic involute's does.

The basic involute's constant offset trait between successive spirals also holds true for adjacent curves offset only by $\theta_{0}$, as shown in Fig. 3.4. Not only does this mean that two adjacent curves will enclose an interior space with constant width (the distance between curves), but also that if the process is repeated to encapsulate one entire revolution of the base circle, then the resulting curves will all maintain equal and constant separation from their neighbors. 


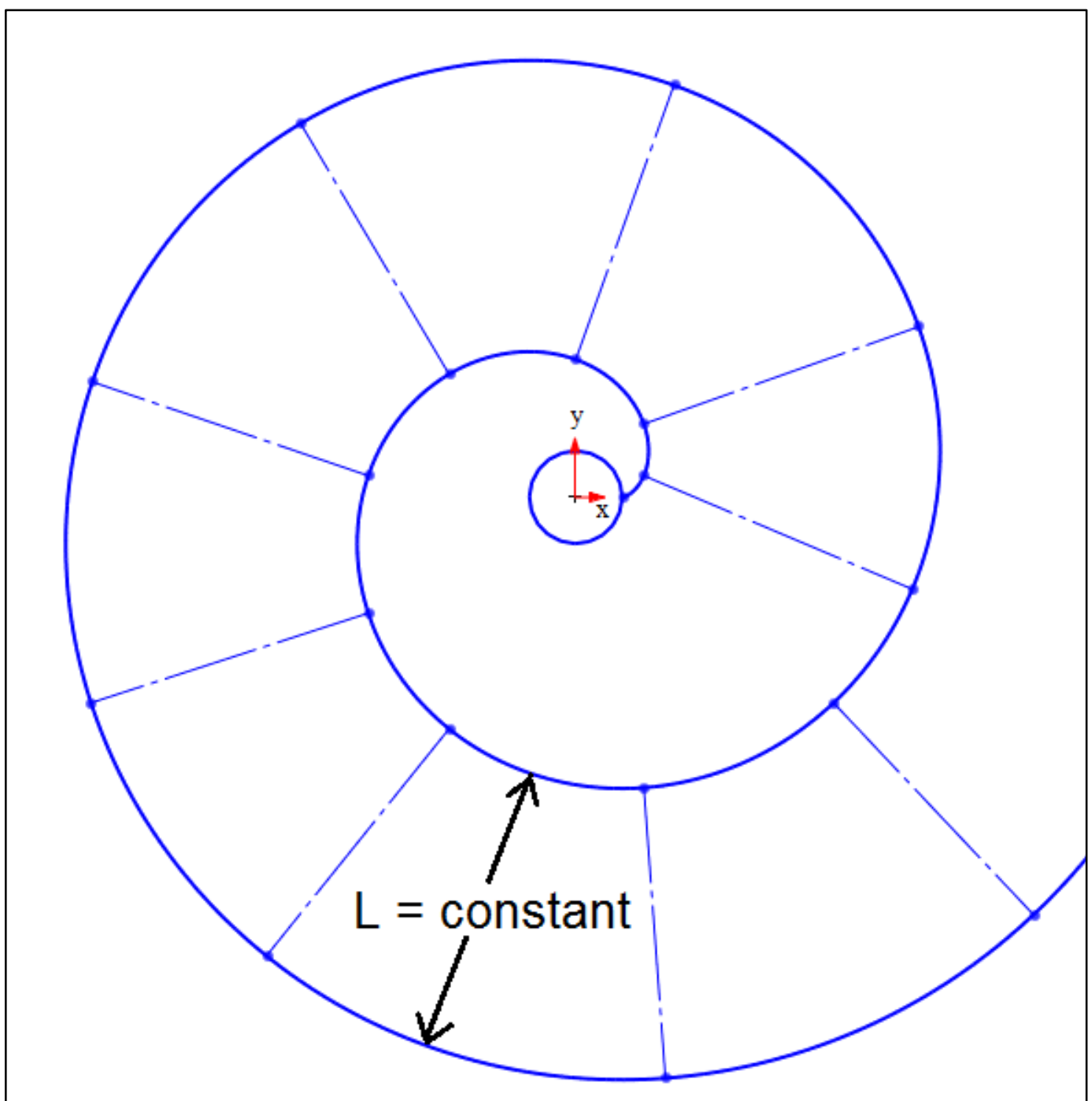

Fig. 3.3. Involute Curve Constant Offset.

This process of incrementally increasing the angular offset of each successive curve can be repeated $n$ times for $n$ number of curves.

$$
\begin{gathered}
x_{i}=r_{b}\left[\cos \left(\theta_{i}\right)+\alpha \sin \left(\theta_{i}\right)\right] \quad \alpha \geq 0, \quad i=1,2,3, \ldots, n \\
y_{i}=r_{b}\left[\sin \left(\theta_{i}\right)-\alpha \cos \left(\theta_{i}\right)\right], \alpha \geq 0, \quad i=1,2,3, \ldots, n \\
\theta_{i}=\alpha+2 \pi \frac{i-1}{n}, \quad \alpha \geq 0, \quad i=1, \quad 2,3, \ldots, n .
\end{gathered}
$$




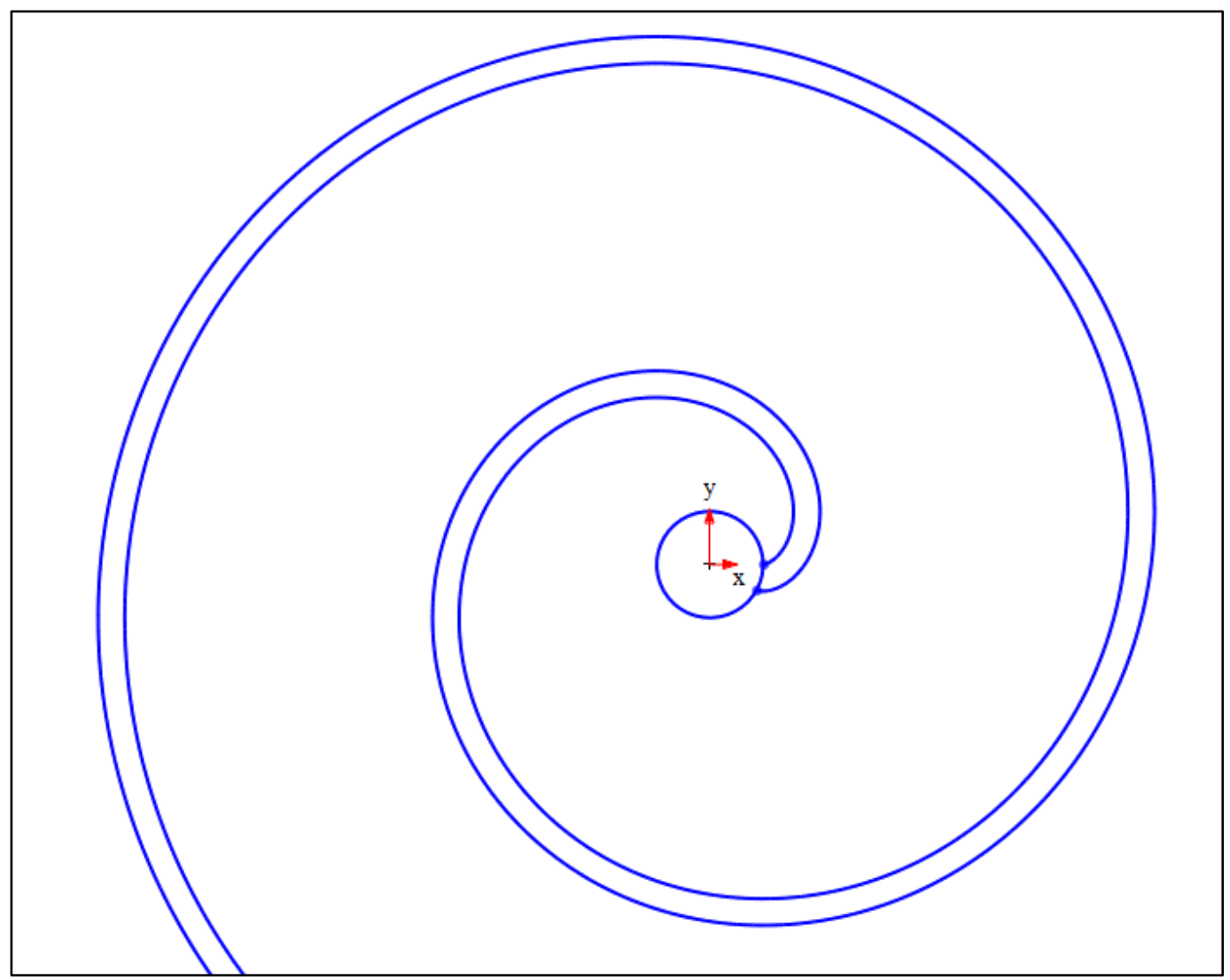

Fig. 3.4. Adjacent Involute Constant Offset. The curve with the upper origin point has $\alpha=\theta$ where $\theta_{\mathrm{o}}=0$. The curve with the lower origin has $\alpha=\theta+1 / 2$ where $\theta_{\mathrm{o}}=-1 / 2$.

The HFIR core consists of 540 basic involute fuel plates, 171 of which are arrayed in the inner element. The HFIR inner core can be recreated with $n=342$. This is twice the number of fuel plates since each plate is separated by a coolant channel. This produces 171 constant-width fuel plates and 171 constant-width coolant channels. A magnified slice of the geometry that this produces is shown in Fig. 3.5 . 


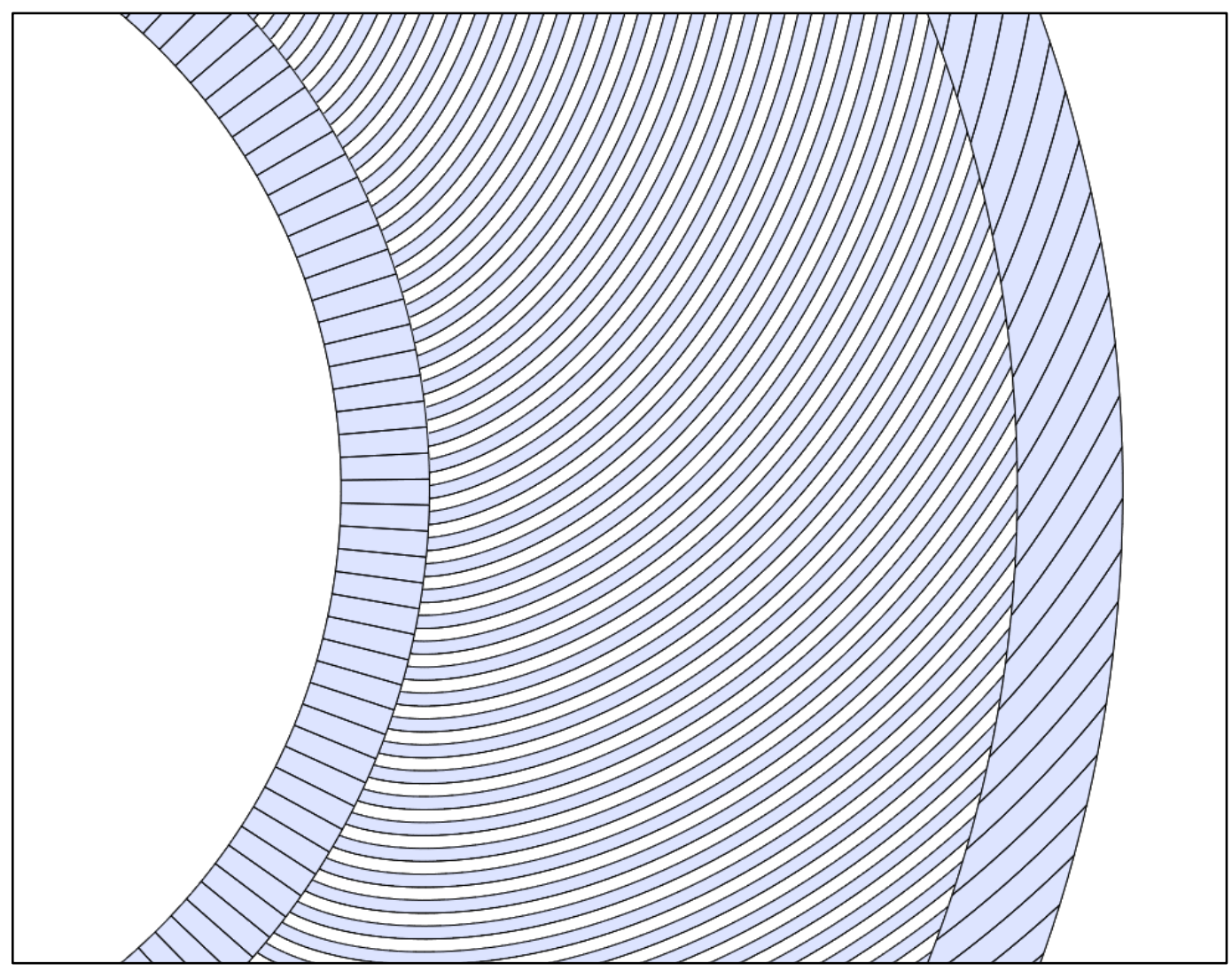

Fig. 3.5. HFIR Core Inner Element Fuel Plate Configuration. Inner element involute fuel plates are shown in grey with inner and outer side plates capping each end. The alternating white spaces between fuel plates are coolant channels.

\section{B. Select Alterations to the Basic Involute:}

The fact that each fuel plate and coolant channel has a constant width is vital to HFIR's performance capabilities. Of the five remaining domestic research reactors not yet converted to LEU fuel under the GTRI, HFIR is the only one with involute fuel plate geometries in its core. The Advanced Test Reactor at Idaho National Laboratory, for instance, uses a four-leaf clover-like array as shown in Fig. $3.6{ }^{55}$ The MIT reactor core has a hexagonal cross-section composed of rhomboidal elements containing linear fuel plates arranged with different orientations, as shown in Fig. 3.7. While these alternate fuel configurations undoubtedly have advantages - either in performance criteria or otherwise — of their own, they lack the axial symmetry of the HFIR core configuration. Under ideal conditions - that is without one-off fuel defects or single-channel flow blockages - each inner fuel plate will experience the exact same operating conditions as every other inner fuel plate. It is a trade of sorts between the complexity of the geometry and the symmetry this produces in the reactor core's output. This uniformity allows the reactor to utilize 
its available fuel source more efficiently. While there are still radially-dependent variances in each plate's output, the fact that they are constant between all plates means that each plate can be burned via nuclear fission equally. This allows the entire HFIR core to be replaced at once, rather than in segments dependent upon each fuel element's position in the reactor, as with the two reactors depicted below.

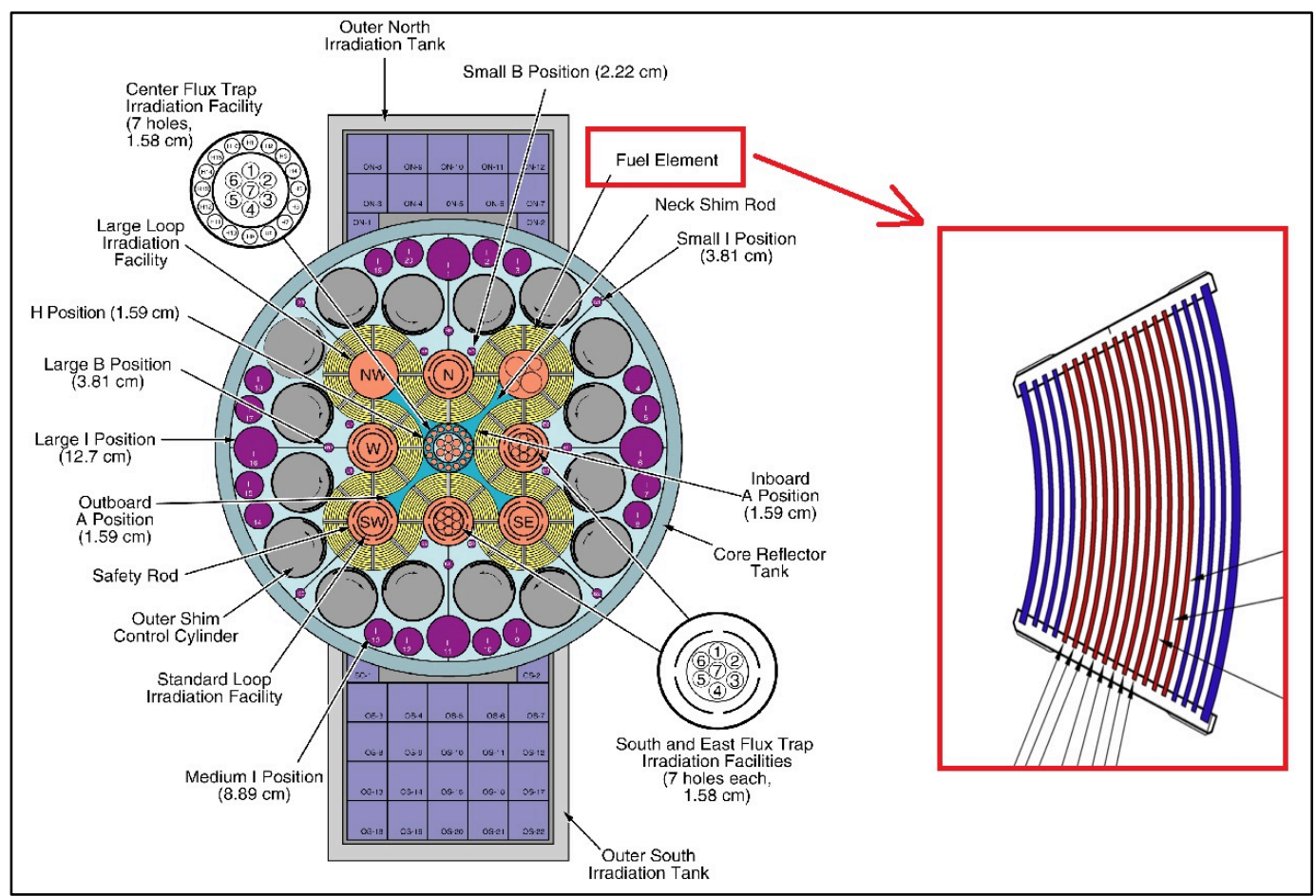

Fig. 3.6. ATR Core Cross-Section and Fuel Plate Detail. ${ }^{56}$ The ATR core only maintains quarter-turn radial symmetry. As shown in the detail of a typical fuel plate configuration on the right, the plate's position dictates a variance not only in length but also in thickness.

Developing a simplified means of considering the complex geometry in the HFIR fuel plates was the first hurtle in modeling the core. While Eqn. (3.4) adequately describes any general involute curve, it is not sufficient for describing the internal fuel geometries of the plates nor does it lend itself to a practical reference frame. For these purposes, Eqn. (3.4) can be rewritten in terms of two involute coordinates, $\theta$ and $t$. The new involute variable $t$ is the perpendicular offset distance from a base involute curve. It takes the place of $\alpha$ such that

$$
\alpha(\theta)=\theta+\frac{t}{r_{b}}, \quad \alpha \geq 0
$$


where $t$ is measured positive extending out from the convex side of the curve and negative out from the concave side.

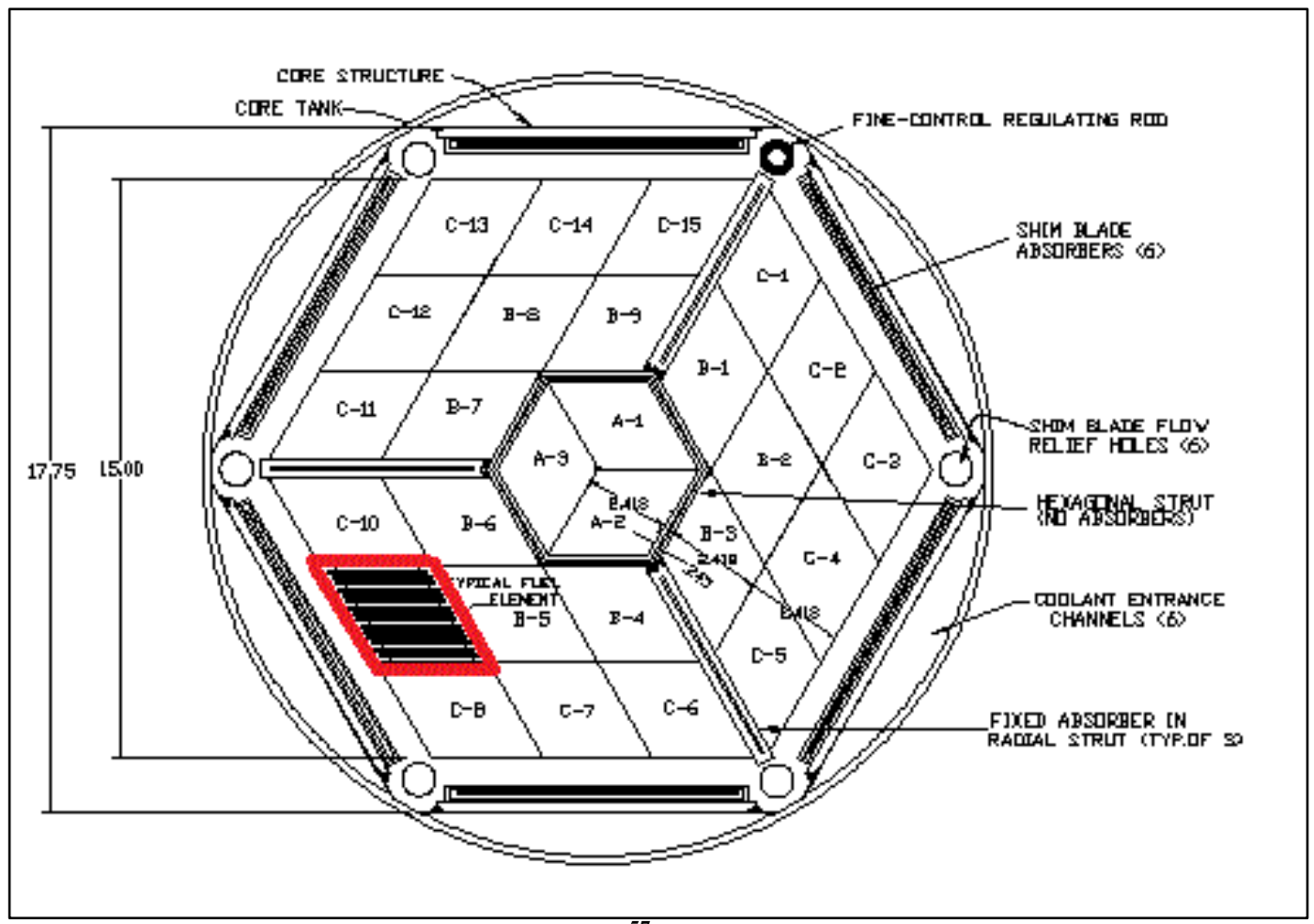

Fig. 3.7. MIT Reactor Core Cross-Section. ${ }^{57}$ The MIT reactor core only maintains one-thirdturn radial symmetry.

A pair of curves in which $t$ is set to equal 0 and $r_{b}$ such that $\theta$ equals $\alpha$ and $\alpha+1$, respectively, is shown in Fig. 3.8. Both the arc length along the circumference of the base circle, $a$, and the perpendicular offset distance, $t$, between the two involute curves equals $r_{b}$. Thus it can be said that

$$
\frac{a}{r_{b}}=\frac{t}{r_{b}}=\theta_{o}
$$




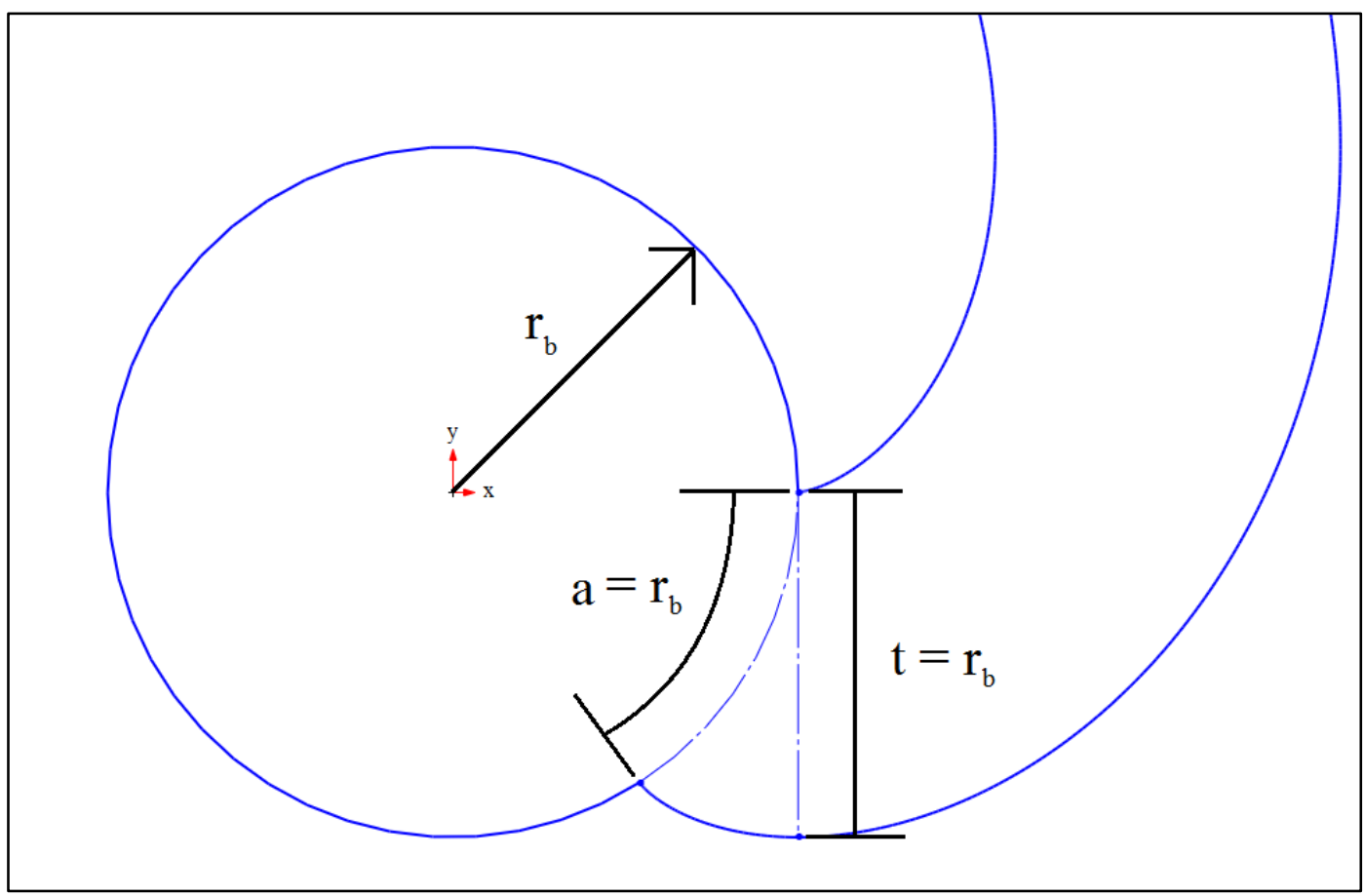

Fig. 3.8. Perpendicular Involute Offset. The top involute curve has $\theta=\alpha$. The bottom involute curve has $\theta=\alpha+1$ which corresponds to a $\theta_{o}$ of 1 radian (CW positive).

In this new reference frame, adjacent involute curves are generated by specifying $t$, the linear offset distance between them, rather than the angular offset between their origin points. Eqn. (3.7) is valid for any involute with a constant offset. For involute curves with non-constant offset, $t$ can be specified as a function of $\theta$. If $t$ varies linearly with $\theta$, the offset can be determined using point-slope form such that

$$
\begin{gathered}
\alpha(\theta)=\theta+\frac{t(\theta)}{r_{b}}, \quad \alpha \geq 0, \quad t \ll r_{b} \\
t(\theta)=\frac{t_{2}-t_{1}}{\theta_{2}-\theta_{1}}\left(\theta-\theta_{1}\right)+t_{1}
\end{gathered}
$$

where $\theta_{1}$ and $\theta_{2}$ are the start and end points of the sloped line, respectively, and $t_{1}=t\left(\theta_{1}\right), t_{2}=t\left(\theta_{2}\right)$. If $t$ is given as a smooth, continuous function of $\theta$ then Eqn. (3.9a) can be used independently.

In the same way that $t$ is a more readily accessible substitute for $\theta_{o}$, the angular coordinate $\theta$ can be converted into arc length along the involute curve, $s$. The variable $t$ will be assumed constant in the following relations. First, consider the basic definition of arc length 


$$
s_{a \rightarrow b}=\int_{a}^{b} d s .
$$

For the parametric equation given by Eqns. (3.4) and (3.7), the quantity $d s$ is represented by

$$
d s=\sqrt{\left(\frac{d x}{d \theta}\right)^{2}+\left(\frac{d y}{d \theta}\right)^{2}} d \theta
$$

Differentiating Eqns. (3.4) and (3.7) with respect to $\theta$, we obtain

$$
\begin{aligned}
& \frac{d x}{d \theta}=\left(r_{b} \theta+t\right) \cos \theta \\
& \frac{d y}{d \theta}=\left(r_{b} \theta+t\right) \sin \theta .
\end{aligned}
$$

Substituting into Eqn. (3.11) produces

$$
d s=\sqrt{\left(r_{b} \theta+t\right)^{2}\left(\cos ^{2} \theta+\sin ^{2} \theta\right)} d \theta
$$

Using the Pythagorean identity ${ }^{58}$, this can be simplified to

$$
d s=\left(r_{b} \theta+t\right) d \theta
$$

Substituting into Eqn. (3.10) yields

$$
S_{a \rightarrow b}=\int_{a}^{b}\left(r_{b} \theta+t\right) d \theta
$$

and integrating, produces

$$
s_{a \rightarrow b}=\left[\frac{r_{b} \theta^{2}}{2}+t \theta\right]_{a}^{b} .
$$

Assuming $\theta_{\mathrm{a}}=s_{\mathrm{a}}=0$ as a universal point of reference, this becomes

$$
s(\theta)=\frac{r_{b} \theta^{2}}{2}+t \theta, \quad \theta \geq 0
$$


Arc length is considered a superior alternative to $\theta$ due to the fact that it increases linearly along the span of the fuel plate whereas $\theta$ increases at a rate proportional to the square root of $s$. In addition to $s$ and $\theta$ there is a third coordinate, radius, $r$, that is frequently used to define positions along the span of the fuel plate. As various inputs and references use a mixture of all three coordinates, it is important to be able to easily and readily convert between the three. Radial position, $r$, can easily be determined as a function of $\theta$ by starting with

$$
r=\sqrt{x^{2}+y^{2}}
$$

Substituting Eqns. (3.4) and (3.7) into Eqn. (3.18) yields

$$
r=\sqrt{r_{b}^{2}\left[\cos \theta+\left(\theta+\frac{t}{r_{b}}\right) \sin \theta\right]^{2}+\left[\sin \theta-\left(\theta+\frac{t}{r_{b}}\right) \cos \theta\right]^{2}}, \theta \geq 0
$$

Squaring the quantities allows for the grouping of like terms

$$
r=r_{b} \sqrt{\left(\cos ^{2} \theta+\sin ^{2} \theta\right)+\left(\theta+\frac{t}{r_{b}}\right)^{2}\left(\cos ^{2} \theta+\sin ^{2} \theta\right)+2\left(\theta+\frac{t}{r_{b}}\right)(\cos \theta \sin \theta-\cos \theta \sin \theta)}
$$

$\theta \geq 0$

Again using the Pythagorean identity and cancelling like terms produces $r$ as a function of $\theta$

$$
r(\theta)=r_{b} \sqrt{\left(\theta+\frac{t}{r_{b}}\right)^{2}+1}, \quad \theta \geq 0
$$

To bring the reference frame relations full circle, $s$ can also be solved as a function of $r$. To start, Eqn. (3.21) is solved for $\theta$

$$
\theta(r)=\sqrt{\left(\frac{r}{r_{b}}\right)^{2}-1}-\frac{t}{r_{b}}, r \geq r_{b}
$$

The result is then substituted into Eqn. (3.17) to produce 


$$
s=\frac{r_{b}}{2}\left[\sqrt{\left(\frac{r}{r_{b}}\right)^{2}-1}-\frac{t}{r_{b}}\right]^{2}+t\left[\sqrt{\left(\frac{r}{r_{b}}\right)^{2}-1}-\frac{t}{r_{b}}\right], r \geq r_{b} .
$$

Squaring the first term yields

$$
s=\frac{r_{b}}{2}\left[\left(\frac{r}{r_{b}}\right)^{2}-1-2\left(\frac{t}{r_{b}}\right) \sqrt{\left(\frac{r}{r_{b}}\right)^{2}-1}+\left(\frac{t}{r_{b}}\right)^{2}\right]+t\left[\sqrt{\left(\frac{r}{r_{b}}\right)^{2}-1}-\frac{t}{r_{b}}\right], \quad r \geq r_{b} .
$$

Multiplying through and grouping like terms produces

$$
s=\frac{r^{2}}{2 r_{b}}-\frac{r_{b}}{2}+(t-t) \sqrt{\left(\frac{r}{r_{b}}\right)^{2}-1}+\frac{t^{2}}{r_{b}}\left(\frac{1}{2}-1\right), r \geq r_{b}
$$

which reduces to

$$
s=\frac{r^{2}}{2 r_{b}}-\frac{r_{b}}{2}-\frac{t^{2}}{2 r_{b}}, \quad r \geq r_{b}
$$

Simplifying yields

$$
s(r)=\frac{1}{2 r_{b}}\left[r^{2}-r_{b}^{2}-t^{2}\right] r \geq r_{b}
$$

With Eqns. (3.17), (3.21), and (3.27) it is possible to quickly cycle among all three coordinate systems. The relations for $s(r), r(\theta)$, and $s(\theta)$ can readily be solved in reverse, allowing for quick conversions between any of the three primary involute coordinates.

The final alteration to the basic involute equation that is required in order to recreate specific HFIR fuel configurations is the out-of-plane alteration for involute curves with an axial component. The basic involute curve, referred to, in this context, as the in-plane involute, and all of its alterations presented thus far, exists solely in a two-dimensional radial plane that is perpendicular to the axis of the core. The question arises of how to most appropriately extend the involute into the axial dimension to create an outof-plane involute. There are many choices for defining the out-of-plane involute's axial position. Primarily, one could define the axial position as a function of $\theta, r$, or $s$. One could also envision the outof-plane involute as the hypotenuse of an involute triangle with the in-plane involute and the axial line connecting their two ends completing the perimeter of the triangle. 
The most physically appropriate answer to this question comes from the fabrication process used to create the plates. The fuel plates, consisting of aluminum cladding and enriched uranium fuel powder, are assembled in a flat, rectangular geometry. The involute curve is introduced by pressing the flattened assembly against an involute mold. In envisioning this process, it is easy to understand that the fuel plate is bent as a function of arc length along the in-plane involute curve. In the same way, the extension of the involute curve into the axial direction should be a function of arc length in order to realistically approximate the effects of this real-world bending process.

The introduction of an axial component into an involute curve in this manner turns out to be fairly simple. Consider again Eqns. (3.4) and (3.7). Let them represent a generic in-plane involute curve. A third equation to represent the curve's axial position as a function of the variable $\theta$ must be added to this set. The new parametric set then becomes Eqns. (3.4), (3.7), and

$$
z(\theta)=\frac{z_{2}-z_{1}}{s_{2}-s_{1}}\left(s(\theta)-s_{1}\right)+z_{1}
$$

where $s(\theta)$ is given by Eqn. (3.17). The resultant out-of-plane involute curve that this produces is shown in Fig. 3.9.

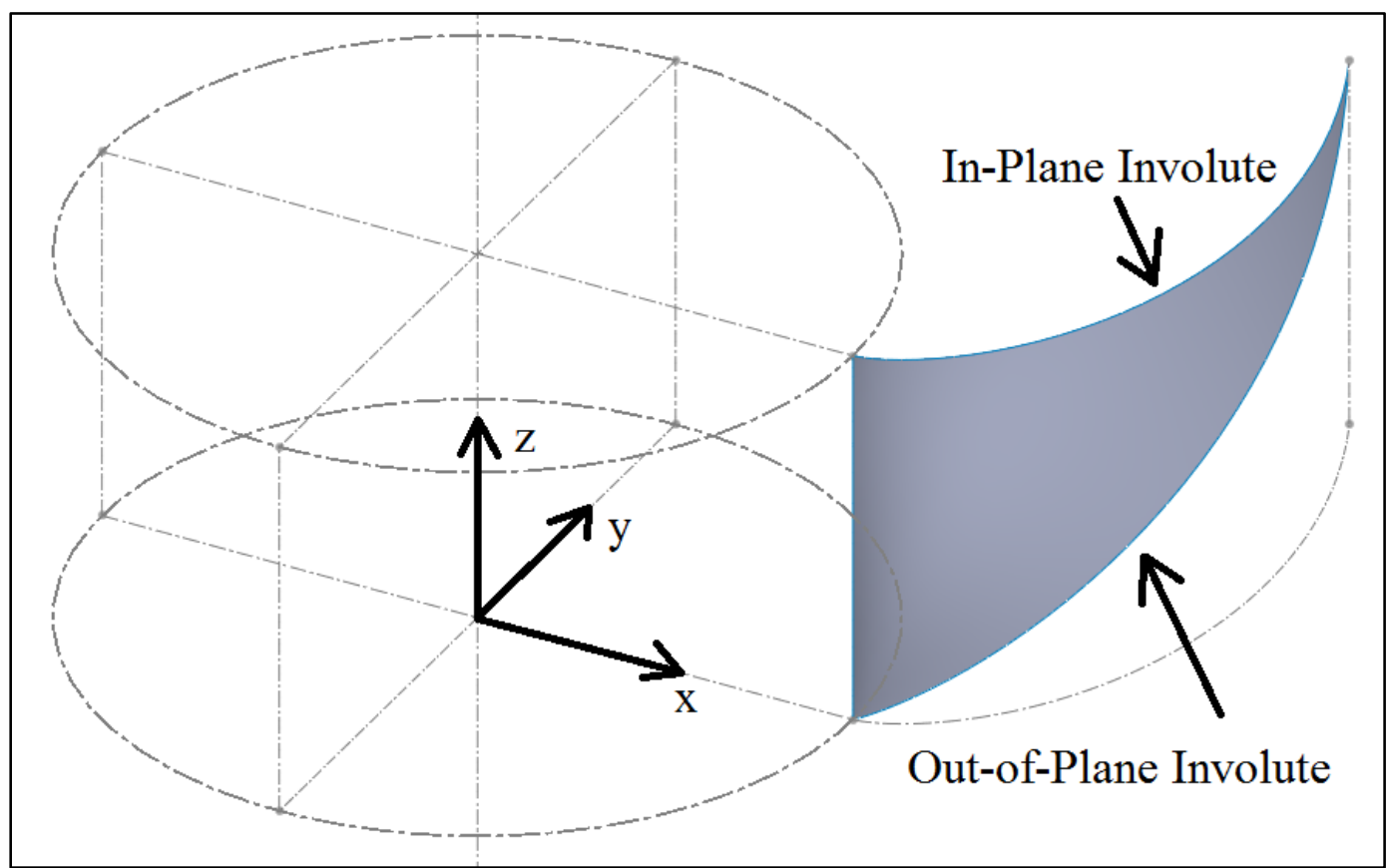

Fig. 3.9. Out-of-Plane Involute Curve. The $z$-direction denotes the direction of the axis. As viewed down the axis, the out-of-plane involute has the same footprint as its in-plane involute. 


\section{Geometry Creation}

For the purposes of this thesis, and due to the fact that the inner element has traditionally produced the most extreme temperature field during HEU operation, only inner element geometries will be considered. The most basic involute geometry in the core is that of the aluminum fuel plate. Its basic dimensions are shown in Fig. 3.10. The fuel plate's primary distinguishing features are a rounded leading edge and a chamfered inner corner. Given that this second feature is obscured from the coolant channel during operation, however, it can be removed from the CAD construction. In models in which the side plates are included for the purpose of analyzing heat transfer into them from the coolant and fuel plate, the fuel plate itself is cut off at this overlap boundary. Anything beyond this line is assumed to be side plate - a trivial distinction given the uniformity of material used to construct both the fuel plates and the side plates.

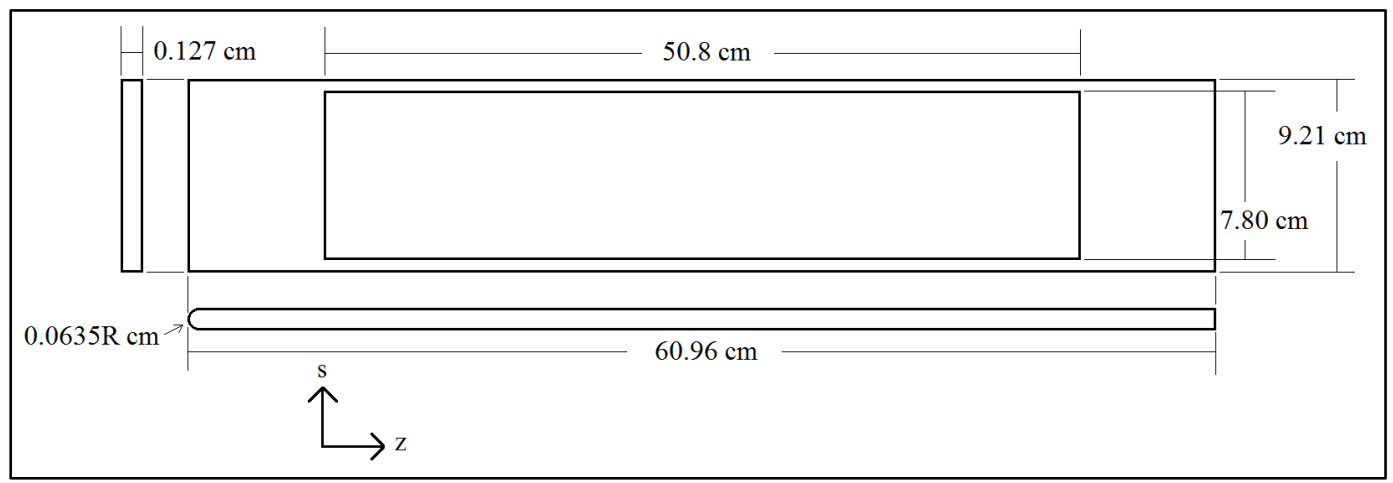

Fig. 3.10. Inner Element Fuel Plate Dimensions.

In considering the "basic" fuel plate, one is considering only the external dimensions. In actuality, the fuel plate has an internal cavity in which the fuel meat is placed. This cavity can be created through a Boolean subtraction of the fuel meat geometry from the solid fuel plate geometry, however, rather than through manual CAD construction. For the fuel meat itself, three geometries will be considered in this thesis. The first is the existing HEU fuel meat. The HEU fuel has a thickness that varies as a smooth function of $s$. The HEU fuel is axially consistent, meaning that its profile in the $s$ - $t$ plane remains constant for all axial positions. All of the fuel meats are non-symmetric such that one side forms a datum at $t=0$. The contour of the other side thus varies directly with the thickness of the fuel meat. The HEU fuel meat thickness profile is shown in Fig. 3.11. The profile is shown in linearized form in which the $s$ - $t$ plane has been mapped to an $x-y$ Cartesian grid using a 1:1 mapping. 


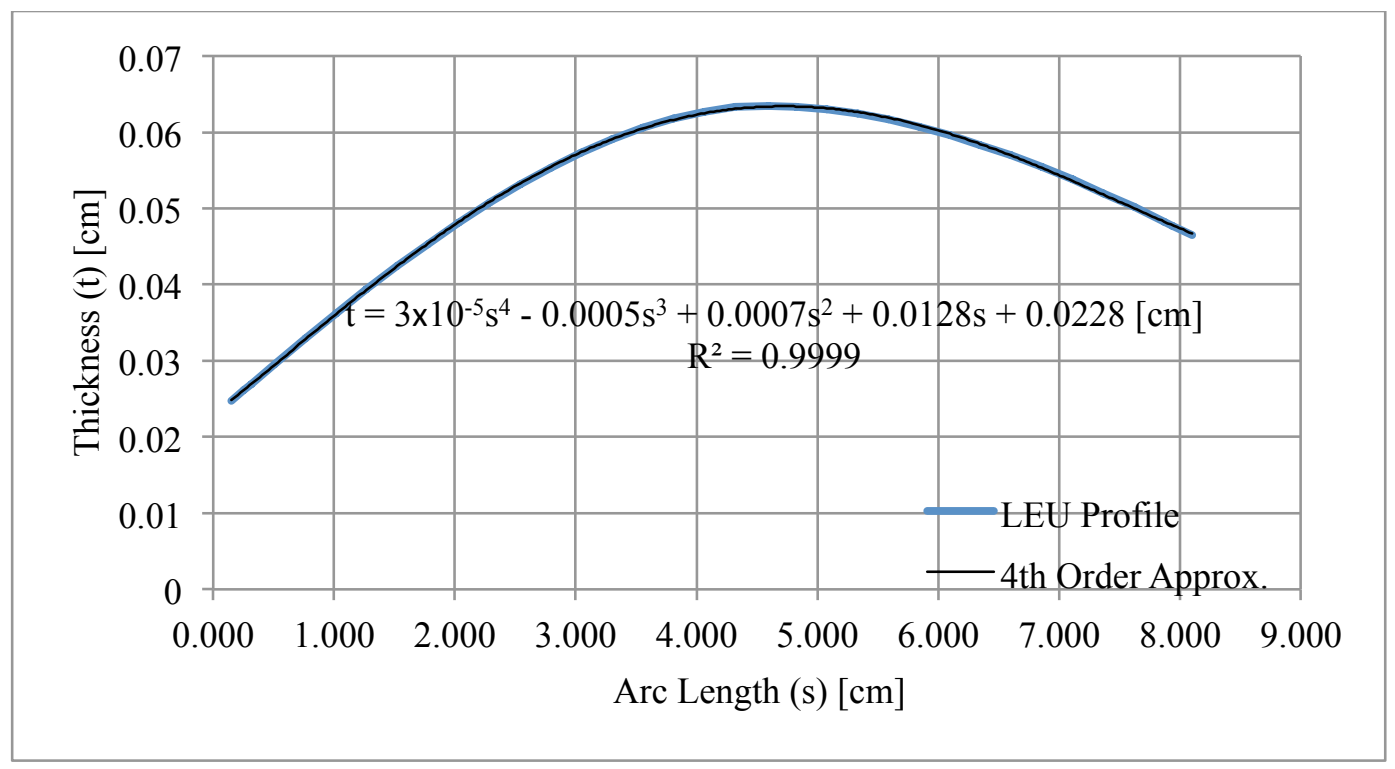

Fig. 3.11. Linearized HEU Fuel Thickness Profile. The curve fit is generated from 35 unevenly distributed points.

The HEU thickness is given as a collection of points that can be represented in $\mathrm{cm}$ by the $4^{\text {th }}$ order polynomial

$$
t(s)=\left(3 s^{4}-50 s^{3}+70 s^{2}+1280 s+2280\right) \times 10^{-5}, \quad 0.152 \leq s \leq 8.103
$$

with a coefficient of determination, $\mathrm{R}^{2}$, of 0.9999 indicating a close approximation of the actual HEU thickness profile. The coefficient of determination is given by ${ }^{59}$

$$
R^{2}=1-\frac{\sum_{i=1}^{35}\left(t_{i}-f\left(s_{i}\right)\right)^{2}}{\sum_{i=1}^{35}\left(t_{i}-\bar{t}\right)^{2}}
$$

where $t_{i}$ is the collection of points used to define the HEU thickness profile, $f\left(s_{i}\right)$ is the value of the approximation at those points, and $\bar{t}$ is the average of the defining points.

The second geometry is an intermediate LEU fuel meat. The intermediate LEU fuel has a thickness that varies piecewise linearly with $s$ and is also axially consistent. The linearized LEU thickness profile is shown in Fig. 3.12. 


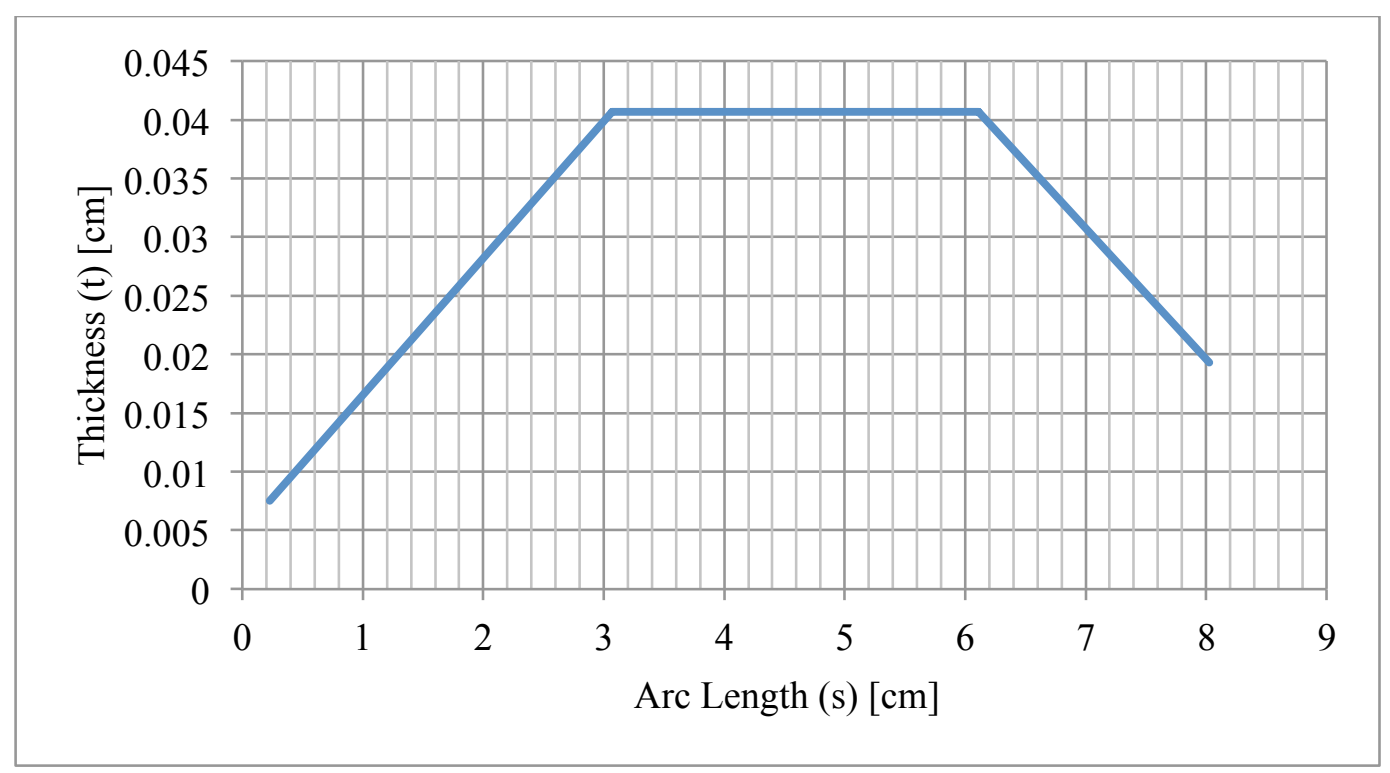

Fig. 3.12. Linearized LEU Fuel Thickness Profile. Overall, the LEU fuel profile is thinner than the HEU profile. Even though the LEU fuel has less $U^{235}$ per weight than the HEU fuel, it has a higher density.

The LEU thickness in $\mathrm{cm}$ can be represented by the piecewise function

$$
t(s)=\left\{\begin{array}{ccc}
0.1168 s+0.0102, & \text { if } & 0.2309 \leq s \leq 3.0270 \\
0.0407, & \text { if } & 3.0720 \leq s \leq 6.1100 \\
-0.0112 s+0.1027, & \text { if } & 6.1100 \leq s \leq 8.0270
\end{array}\right.
$$

The third geometry is another LEU fuel meat with the same starting profile as the first, but with the inclusion of axial grading. This geometry tapers towards its trailing edge in an effort to prevent a sharp spike in nuclear heat energy output in the tail of the fuel meat. This third geometry was, at the onset of this study, the geometry proposed for production. Since that time, however, more viable alternative fuel geometries have been proposed. The intermediate LEU fuel is included only because of the relative simplicity and ease of modeling that accompanies the change to an axially-consistent geometry. The axially-graded LEU geometry is shown alongside the intermediate geometry in Fig. 3.13. 


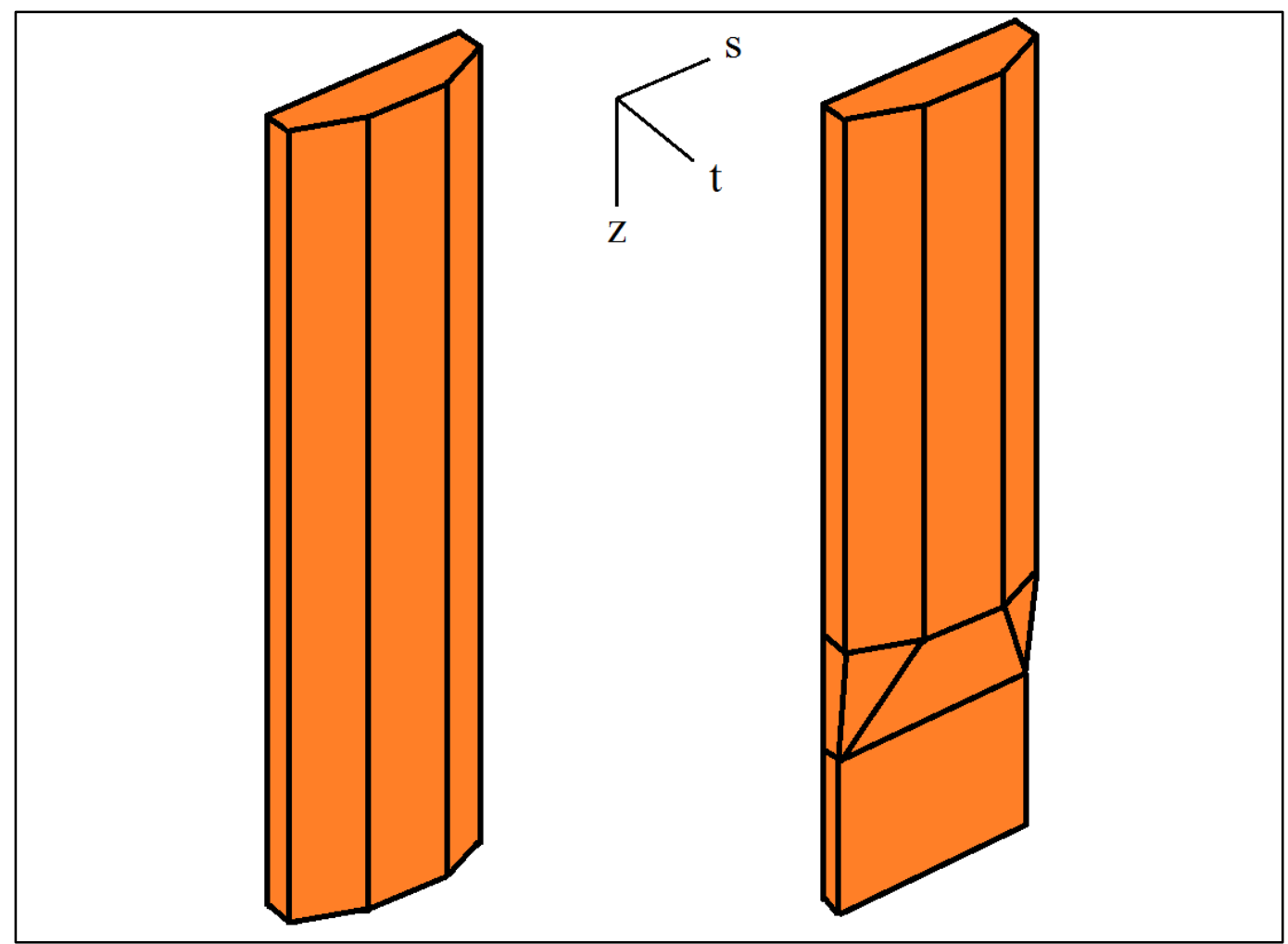

Fig. 3.13. Linearized LEU Fuel Geometries. The axially-consistent intermediate LEU geometry is shown on the left. The axially-graded LEU geometry is shown on the right.

In the process of manufacturing the HEU plate, the cavity in the plate is filled with fuel powder to create the necessary profile. The remainder of the void is then filled with a radioactively inert composition of "filler" powder. The filler is composed by weight of $\sim 99.9 \%$ aluminum powder and $\sim 0.01 \%$ boron poison. ${ }^{7}$ Since the poison constitutes such a small percentage of the overall filler material it can be disregarded with respect to its thermal properties. The filler can be assumed to have the thermal properties of aluminum. Furthermore, since the powder is primarily aluminum, this entire filler region can be assumed to be a part of the fuel plate itself. The only loss is in the modeling of the contact resistance between the two as well as within the powder itself. The final method of manufacturing LEU plates has not been set. Therefore, the same assumptions are used in the LEU model. Under these assumptions, the process of creating the finalized fuel plate geometry through a Boolean subtraction of the fuel meat geometry is valid.

The last major geometry component is that of the coolant. The coolant is, like the fuel plate itself, fairly simple to construct. It encapsulates the fuel plate on the top and bottom as well as the concave and convex sides. An entry region approximately $2.0 \mathrm{~cm}$ in length, corresponding to the top of the side plates, 
is inserted upstream of the leading edge. This allows the inlet flow to be specified using the conditions under which it enters the reactor core. The flow then naturally develops as a result of the impingement of the rounded leading edge. A similar region $3.5 \mathrm{~cm}$ in length, corresponding to the bottom of the outer side plate, is inserted downstream of the trailing edge. This region allows the flow to homogenize downstream of the fuel plate before being bounded by outlet flow conditions. The coolant is bounded in the $t$-direction by lines of periodicity.

Some models also take into account the side plates bounding the fuel plate and coolant channel along their inner and outer radii. These geometries, like the coolant channel, are bounded in the $t$-direction by lines of periodicity. The lines of involute periodicity in the coolant channel are extended into the outer side plate. Since the circular involute is undefined at radii less than the base radius - the region in which the inner side plate exists - lines of radial periodicity are used here instead. These periodic boundary lines are shown in Fig. 3.14.

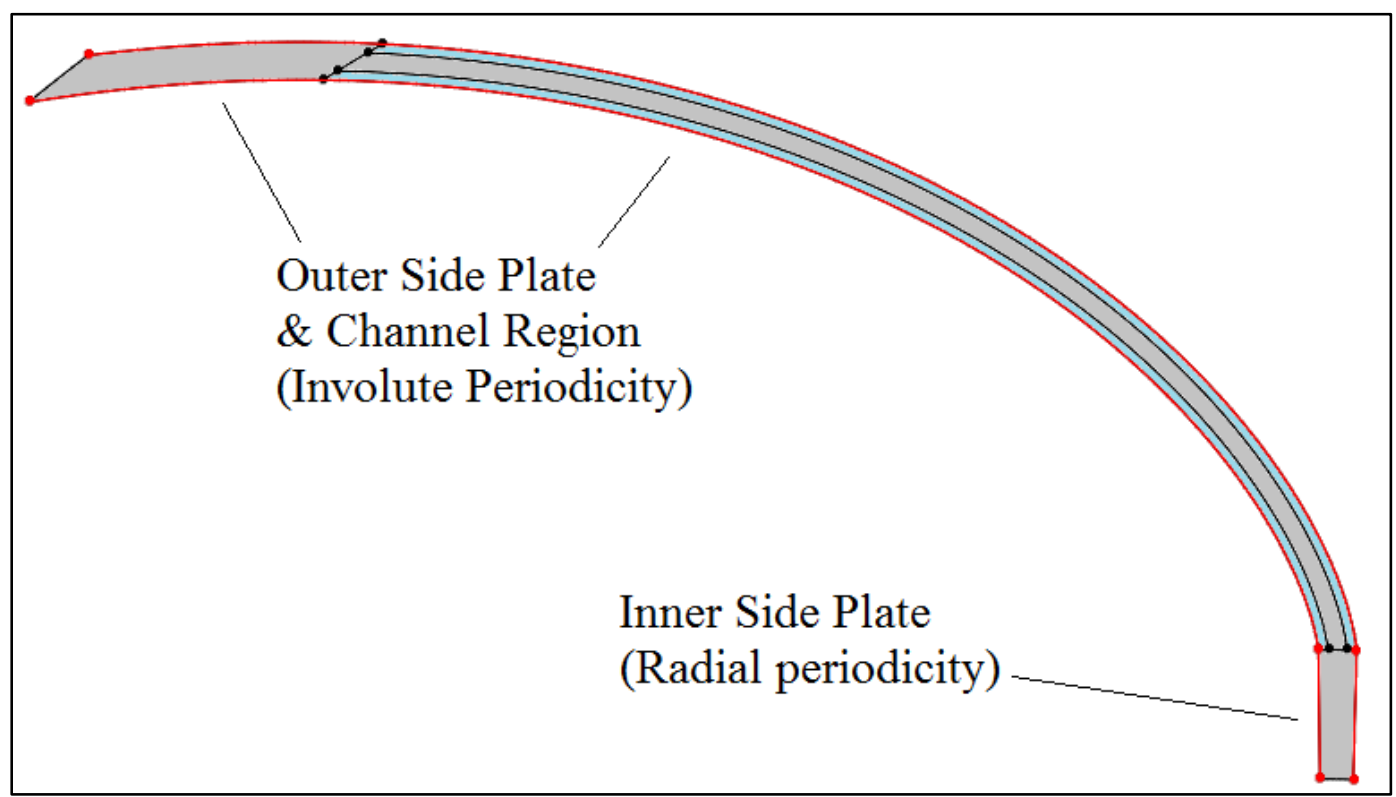

Fig. 3.14. Coolant Channel and Side Plate Periodicity. The fuel plate and side plates are shown in grey. The half coolant channels are shown in blue. Periodic boundaries are highlighted in red.

The side plates' inside and outside boundaries are set according to their bounding radii. These dimensions are given in Table 3.1. 


\begin{tabular}{|c|c|c|}
\hline Table 3.1: & Bounding Side Plate Radii \\
\hline \multirow{2}{*}{ Side Plate } & \multicolumn{2}{|c|}{ Radius } \\
\cline { 2 - 3 } & Inside & Outside \\
\hline Inner & 6.096 & 6.963 \\
\hline Outer & 12.737 & 13.772 \\
\hline
\end{tabular}




\section{COMSOL Overview}

Before simulation work was performed on thesis-related models, software quality assurance (SQA) was performed on COMSOL in accordance with ORNL Research Reactors Division (RRD) requirements. ${ }^{60}$ The process consisted of running example models pre-loaded with the COMSOL distribution on the same machine that thesis-related work would later be performed on. The SQA process was intended as a means of code validation for all HFIR-related LEU conversion work, the scope of this thesis being a subset of a broader modeling and simulation effort. ${ }^{61,62.63}$ Many of the selected example models share, in at least one significant instance, similarities with the work presented in this thesis be it through similar geometry types, domain physics, meshing techniques, or other internal modeling mechanisms. For all cases, solutions to the example models were compared against solutions obtained by COMSOL Multiphysics. In many instances these example models were recreations of published experimental data that had been used by COMSOL Multiphysics as internal benchmarks. For such example models, locally obtained solutions were also compared directly against the original experimental data included in the COMSOL documentation.

COMSOL utilizes the finite element method for the discretization and solution of the domain and the physics modeled therein. The finite element method operates under the assumption that an expansion exists that can perfectly satisfy the governing equations and their boundary conditions in a solution domain. For any solution variable, $\phi$, such an expansion would have the form

$$
\phi(x, y, z)=\sum_{i=1}^{\infty} \phi_{i} \psi_{i}(x, y, z)
$$

where $\psi_{i}$ are basis functions and $\phi_{i}$ are values of the solution variable at specific nodal points. Therefore, for sufficiently large $n$, a finite approximate solution exists such that ${ }^{64}$

$$
\phi(x, y, z) \approx \phi_{n}(x, y, z)=\sum_{i=1}^{n} \phi_{i} \psi_{i}(x, y, z) .
$$

The basis functions most commonly chosen for such large solution domains are linear, quadratic, or cubic. The increase in order carries with it the potential to increase the accuracy of the solution. It also bears the price of increased complexity and computational cost, however, and creates the risk of introducing non-physical oscillations into the final solution, especially in fluid domains. Linear basis functions are usually chosen due to their simplicity, robustness, and decreased computational cost. 
The problems considered in this thesis do not contain known, exact solutions. Therefore, convergence is achieved through the minimization of a residual quantity. The derivation of the residual quantity depends on the governing physical equation for each solution variable. It is essentially a reworking of the governing equation such that evaluating it with the idealized solution from Eqn. (4.1) would yield zero. Evaluating it with the approximate solution, however, will yield a nonzero answer. This quantity is the residual. And so it is the goal of the iterative convergence process to reduce this residual quantity to an acceptable tolerance that is approximately zero.

The total set of solution variables can be formed into a Jacobian solution matrix. Using the accompanying residuals and a set of unknown variables that satisfy the system, the entire domain can be solved using iterative methods. The MUltifrontal massively Parallel sparse direct Solver (MUMPS) ${ }^{65}$ was chosen for both models. The MUMPS solver is a direct solver ideal for large solution domains given its ability to utilize hyper-threading on capable machines and its compatibility with distributed memory server arrays via message passing interface (MPI). The latter ability greatly expands the limiting size and complexity of models.

Even with the available computational resources the iterative solution process was segregated into steps in order to decrease memory requirements. Each iterate was divided into two steps: one to solve temperature, pressure, and velocity and another to solve turbulence variables. Iterate segregation can reduce the stability of the solution process, however, especially if certain "inseparable" variables are segregated separately. To say, for instance, that $T$, as a single variable, was solved in the first step is perhaps an oversimplification. The first step solves $T$ in the solid domain, the fluid domain, and a wall temperature variable on shared surfaces. Attempting to further segregate the model by domain type and moving $T_{\text {solid }}$ into its own step caused the model to diverge. This is readily understandable given the interdependency between all three temperature variables. Likewise, the flow variables $p$ and $u$ are inseparable as are the turbulence variables $k$ and $\varepsilon$. The two-step segregation avoids any such instability concerns while greatly decreasing the memory requirement per iterate by reducing the size of the respective solution matrices.

All thesis-related work was performed on a relative residual tolerance of $1.00 \times 10^{-2}$. This level of tolerance was deemed sufficient due to the fact that increasing it to a higher tolerance yielded only minimal changes to a solution, as will be demonstrated in Section VII. Each solution variable was scaled independently with respect to this residual quantity. These scaling values, shown in Table 4.1, were set manually based on preliminary HFIR-related simulation worked previously performed at ORNL via 
COMSOL. Residuals, $\mathrm{R}$, are calculated for each segregated step based on the $\mathrm{L}_{2}$-norm of the individual scaled residuals in that step given by ${ }^{66}$

$$
|\mathbf{R}|=\sqrt{\sum_{i=1}^{n}\left|\mathrm{R}_{i}\right|^{2}} .
$$

\begin{tabular}{|c|c|}
\hline \multicolumn{2}{|c|}{$\begin{array}{r}\text { Table 4.1: } \\
\text { Scaling Factors }\end{array}$} \\
\hline Variable & Scale \\
\hline $\mathrm{p}$ & $101 \mathrm{kPa}$ \\
\hline $\mathrm{u}$ & $7.77 \mathrm{~m} / \mathrm{s}$ \\
\hline $\mathrm{T}$ & $293 \mathrm{~K}$ \\
\hline $\mathrm{k}$ & $10^{6} \mathrm{~J} / \mathrm{kg}$ \\
\hline$\varepsilon$ & $10^{6} \mathrm{~J} / \mathrm{kg}-\mathrm{s}$ \\
\hline
\end{tabular}




\section{3D Model}

\section{A. Construction}

All geometry pieces were created in SolidWorks and imported into COMSOL for assembly. The assembly process begins with the coolant geometries which are imported first. For ease of meshing the coolant domain is split into six axial components. The model is created with the axial datum $(z=0)$ located at the leading edge of the fuel meat (not the fuel plate itself which is located at $z=-5.08 \mathrm{~cm}$ ). The direction of flow is assumed positive with $z$ increasing down the length of the channel. The coolant channel geometries extend in the $t$-direction to midstream lines of periodicity between adjacent fuel plates. The six coolant components and their axial lengths are listed in Table 5.1.

\begin{tabular}{|c|c|c|}
\hline \multicolumn{3}{|c|}{ Table 5.1: Coolant Components } \\
\hline Component & Axial Region & Length $(\mathrm{cm})$ \\
\hline 1 & Entrance & 2.032 \\
\hline 2 & Leading Edge Cap & 0.178 \\
\hline 3 & Fuel Plate & 60.782 \\
\hline 4 & Trailing Edge Cap & 0.178 \\
\hline 5 & Exit 1 & 1.194 \\
\hline 6 & Exit 2 & 2.311 \\
\hline
\end{tabular}

The exit region is divided into components due to a difference in lengths of the inner and outer side plates which will be added to the model assemblage last. The assembled coolant components are shown in Fig. 5.1.

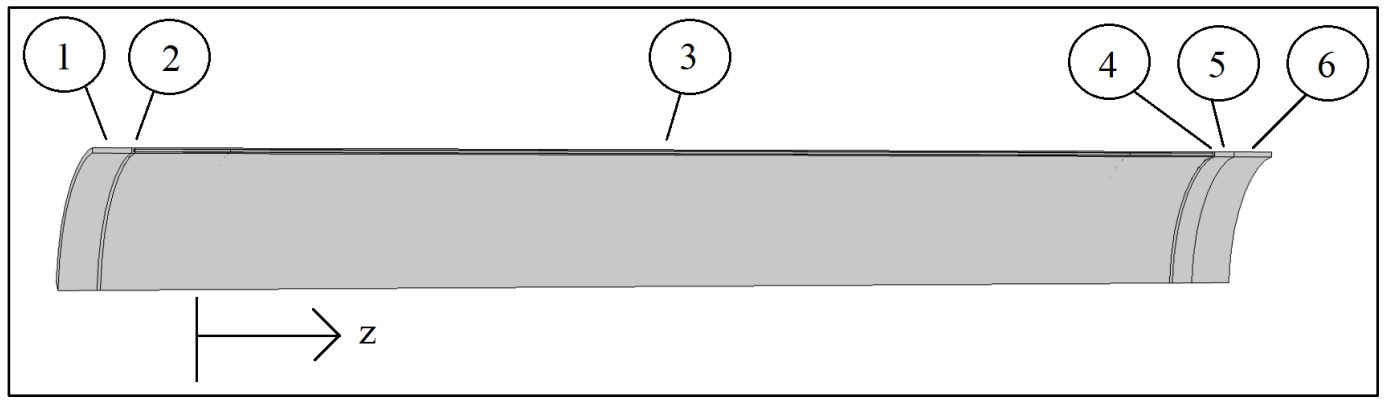

Fig. 5.1. Assembled Coolant Components. Flow direction is in the $+z$-direction. 
Next, the fuel plate components are added to the assemblage. Again for ease of meshing the fuel plate domain is subdivided into four components which are listed in Table. 5.2.

\begin{tabular}{|c|c|c|}
\hline \multicolumn{3}{|c|}{ Table 5.2: Fuel Plate Components } \\
\hline Component & Axial Region & Length $(\mathrm{cm})$ \\
\hline 1 & Leading Edge & 0.089 \\
\hline 2 & Unfueled Top & 4.991 \\
\hline 3 & Fueled Section & 50.800 \\
\hline 4 & Unfueled Bottom & 5.080 \\
\hline
\end{tabular}

The leading edge is separated from the unfueled top region because it is rounded and is easier to mesh separately. The fuel plate components are imported into the COMSOL assemblage and a Boolean subtraction performed in order to remove the overlapping coolant domain. The fuel meat is then imported and subtracted from the fuel plate using the same process. A generalization of the side profile that this produces is shown in Fig. 5.2.

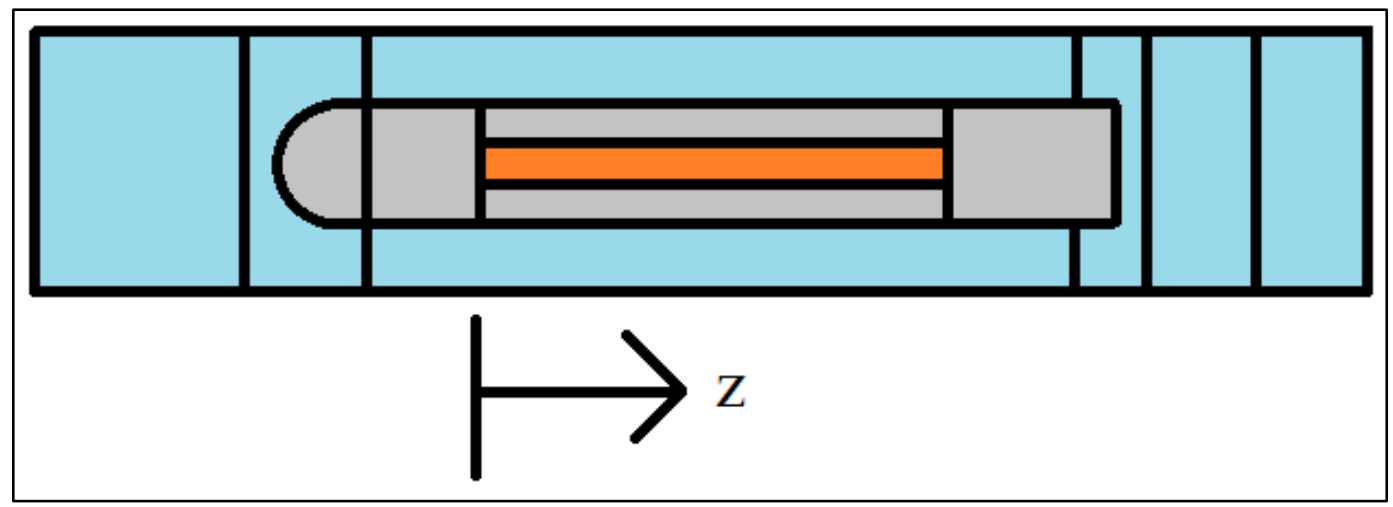

Fig. 5.2. COMSOL Assemblage Side Profile Diagram. Note that the diagram is not to scale.

The coolant channel is shown in blue, the fuel plate in grey, and the fuel meat in orange. While the coolant and fuel plate extend to both side plates, the fuel meat does not.

The side plates are imported last. The inner side plate has an axial length of $64.364 \mathrm{~cm}$ and the outer side plate a length of $66.675 \mathrm{~cm}$. They are aligned equally with their leading edges $2.121 \mathrm{~cm}$ upstream of the fuel plate's leading edge. This is even with the entrance boundary on the top of the leading coolant component. This placement leads both side plates to extend downstream of the fuel plate's trailing edge. 
Their disparity in length dictates that the outer side plate extends $2.311 \mathrm{~cm}$ farther downstream than the inner side plate. The orientation is shown in Fig. 5.3.

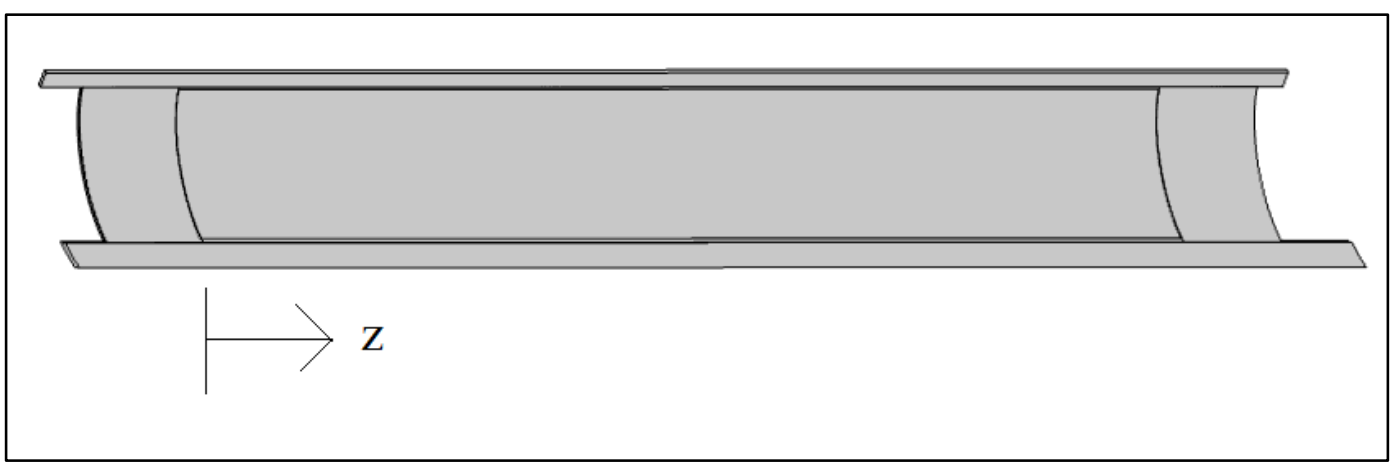

Fig. 5.3. Solid Geometry Assemblage. Flow direction is in the $+z$-direction. The inner side plate is shown on top in this figure. The geometry in between is the fuel plate. Coolant geometry components are not pictured.

This length disparity is an intentional design feature. After a core's operation cycle has ended it is removed from the reactor and placed in the spent fuel pool. Ideally, it is placed on top of a rack allowing ample water to enter into the core from below where natural convection due to heat generated by the leftover fuel expels the water upwards. The side plates are designed such that if a spent core should somehow fall off of its rack and come to rest upright on the floor of the pool, water is able to access the bottom of the core via the vacant flux trap in the center and then be driven upward through the fuel elements via convection. This opening must also be taken into account in the COMSOL model, thus the necessity for coolant components 5 and 6 to exist separately.

With the inclusion of the side plates, the model geometry is completely assembled in COMSOL. The last step is in stitching the geometry components together. COMSOL is capable of doing this in two ways via connections that are known as unions and assemblies. A union is the ideal inter-component connection as it directly links adjacent elements to one another. In a union, any shared face between connected components is considered a single entity. The mesh in one component must match the mesh in the other component at the interface between the two. In an assembly, a shared face between components is considered as two elements, one for each component. For instance, two blocks stacked on top of one another would share a single surface - the bottom of the upper block and the top of the lower block. In an assembly, the top of the lower block and the bottom of the upper block are considered independent of one another. Meshes do not have to match across this interface and physics, such as heat flow, do not 
inherently maintain continuity across it. This can be remedied, however, by placing a boundary pairing on both sides of a shared surface so that continuity is enforced between them.

The assembly interface, while not ideal, can be necessary for complex geometries in which meshing is difficult. Such is the case with some of the components in the fully three-dimensional model with its extremely high-aspect ratio and narrow channels. The effect on results is miniscule. The requirement that the model analyze each assembly interface every iterate, interpolating values and fluxes across its surface from one side to the other and back again, does, however, slightly increase the time per iterate.

Components which can be connected via union are done so. All of the coolant components, for example, can be connected to one another via union as can the fuel meat to the fueled section of the plate. The fueled section, however, must be connected to the unfueled top and bottom of the plate via assembly. This is due to a disparity in meshes on their shared faces.

Given the need for this assembly, the fuel plate cannot be unionized with the coolant channel as one entity. The simplest way to divide them is to create an assembly between the external surface of the fuel plate and the adjacent wall surfaces of the coolant channel. The last required assemblies exist between the side plates and the adjacent coolant channel/fuel plate surfaces.

\section{B. Inputs}

The primary model inputs are composed largely of the reactor design criteria given in Section I. Initial conditions are specified for pressure, temperature, and velocity according to the inlet and outlet conditions in Table 5.3.

\begin{tabular}{|c|c|c|}
\hline \multicolumn{3}{|c|}{ Table 5.3: Initial Inlet and Outlet Conditions } \\
\hline Variable & Inlet & Outlet \\
\hline$p(\mathrm{MPa})$ & 3.33 & 2.59 \\
\hline$T(\mathrm{~K})$ & 322 & 342 \\
\hline$u(\mathrm{~m} / \mathrm{s})$ & 7.77 & 7.77 \\
\hline
\end{tabular}

The initial conditions for pressure and temperature are specified as the linear interpolation between their respective inlet and outlet values. The initial condition for velocity was specified using the constant flow rate relation which states that flow through an area of $\mathrm{A} / 2$ will have a velocity twice that of the same 
flow through an area of A. Given the uniformity in thickness between the fuel plates and coolant channels, the flow area through the fuel section of the reactor core is half the area of the unimpeded section immediately upstream. Initial conditions for pressure, temperature, and coolant velocity as functions of axial position are given in Figs. 5.4, 5.5, and 5.6, respectively. In all three figures dotted red lines indicate extrapolated values. The initial temperature condition was applied to all domains, both solid and liquid. The initial pressure and velocity conditions were applied only to the coolant domain.

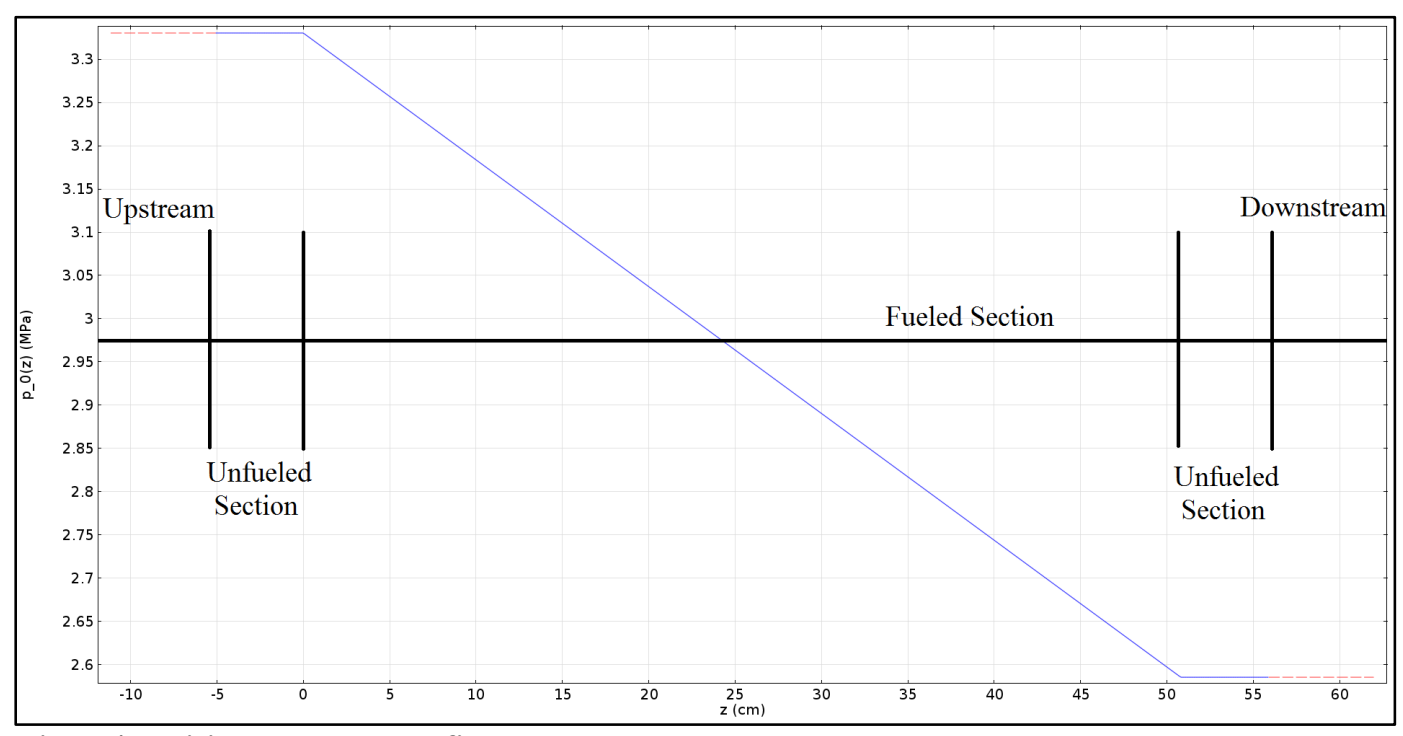

Fig. 5.4. Initial Pressure Profile.

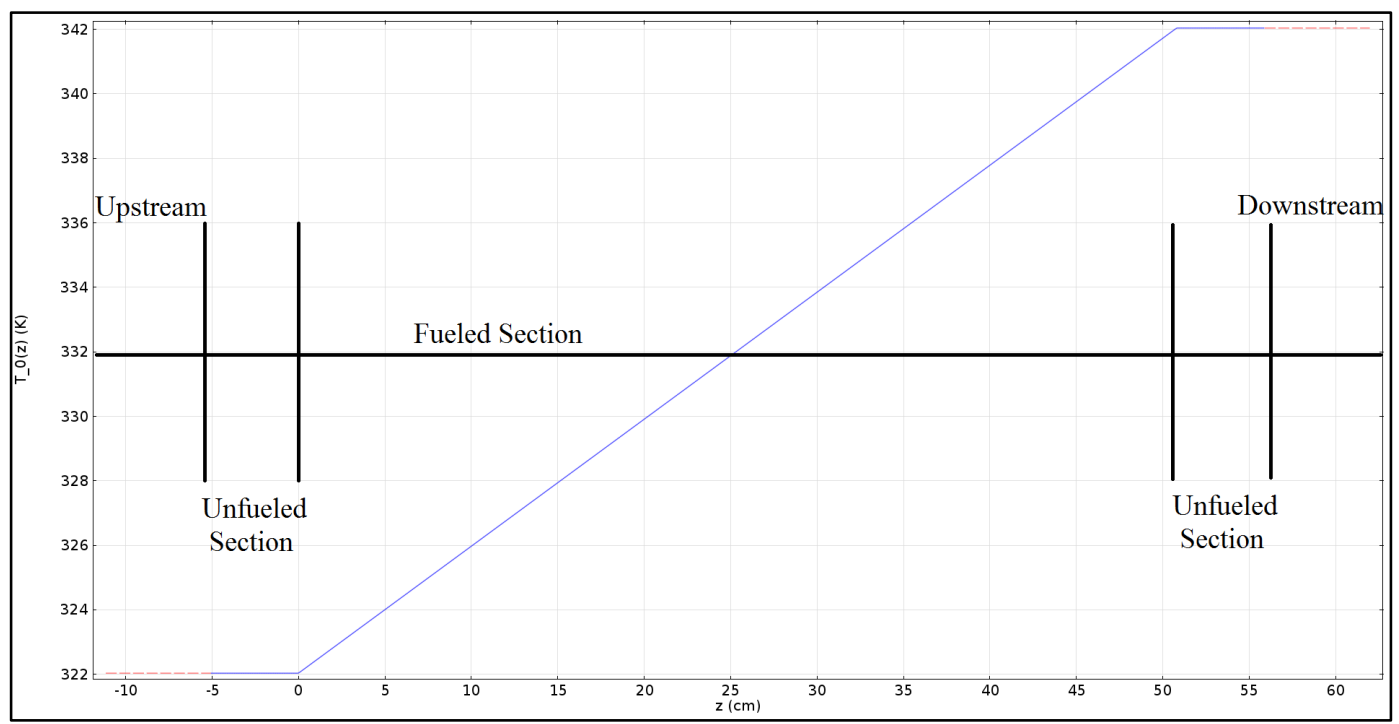

Fig. 5.5. Initial Temperature Profile. 


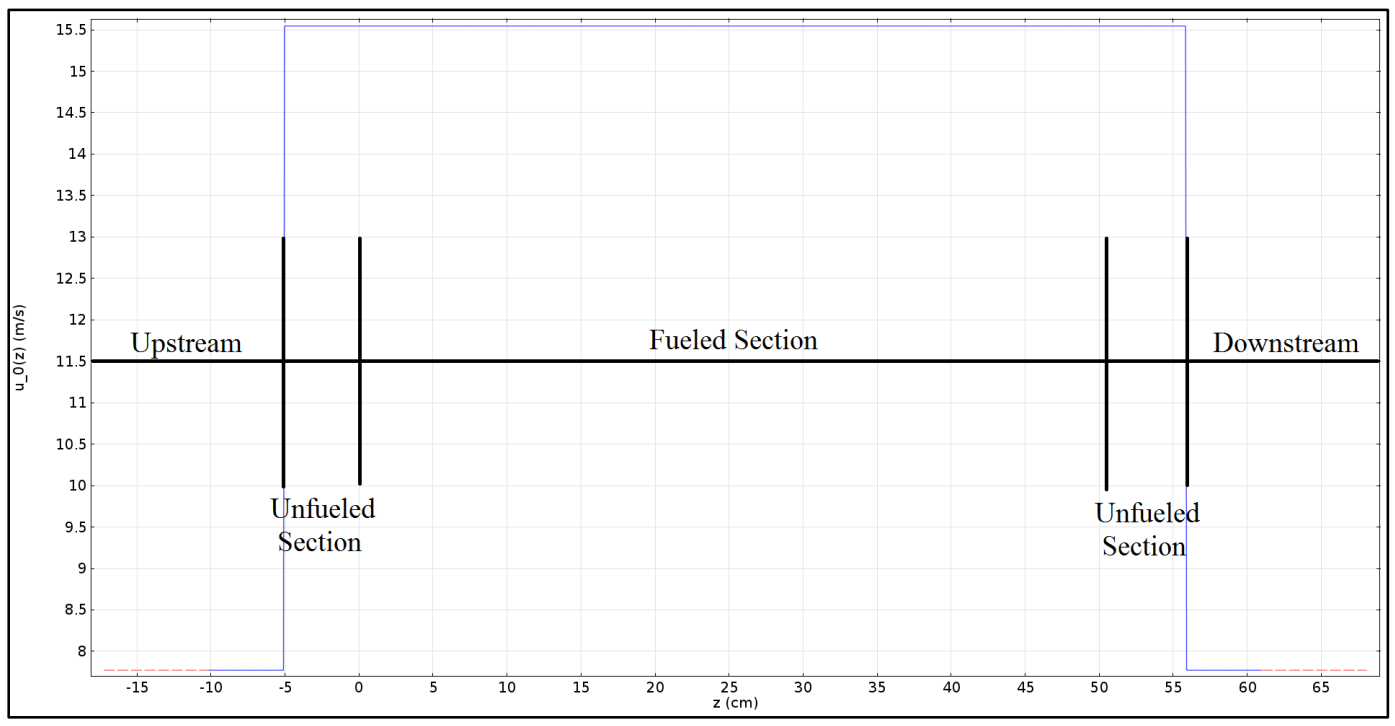

Fig. 5.6. Initial Velocity Profile.

The second model input is the nuclear power generation profile which is divided into two components: a power distribution and fuel thickness factor. The power distribution is a two-dimensional map of LEU beginning-of-cycle (BOC) power density $\left(\mathrm{W} / \mathrm{m}^{3}\right)$ as a function of radial and axial position, $(r, z)$. The power distribution is generated by the Monte Carlo N-Particle (MCNP) transport code. ${ }^{67}$ MCNP is a neutronics code which models the release of nuclear energy throughout the core. It outputs neutron fluxes (neutrons $/ \mathrm{cm}^{2}-\mathrm{s}$ ) as a function of $(r, z)$. These can then be converted into fission rate densities (fissions $/ \mathrm{cm}^{3}$-s) by multiplying by the macroscopic fission cross-section (atoms $/ \mathrm{cm}$ ). Finally, these can be converted into power densities $\left(\mathrm{W} / \mathrm{cm}^{3}\right)$ by multiplying by the amount of energy released per fission reaction ( $\mathrm{J} /$ fission), which is a constant. ${ }^{7}$

MCNP performs this simulation by homogenizing the reactor core internals (fuel meat, aluminum clad, coolant water, etc.) into a single entity and then dividing the core into axisymmetric cells along an $r-z$ grid as shown in Fig. 5.7. As a result of this homogenized grid structure, power density profiles are output in the form of power-per-cell-volume, $P_{\text {cell }}^{\prime \prime \prime}$. The profile is shown in Fig. 5.8. 


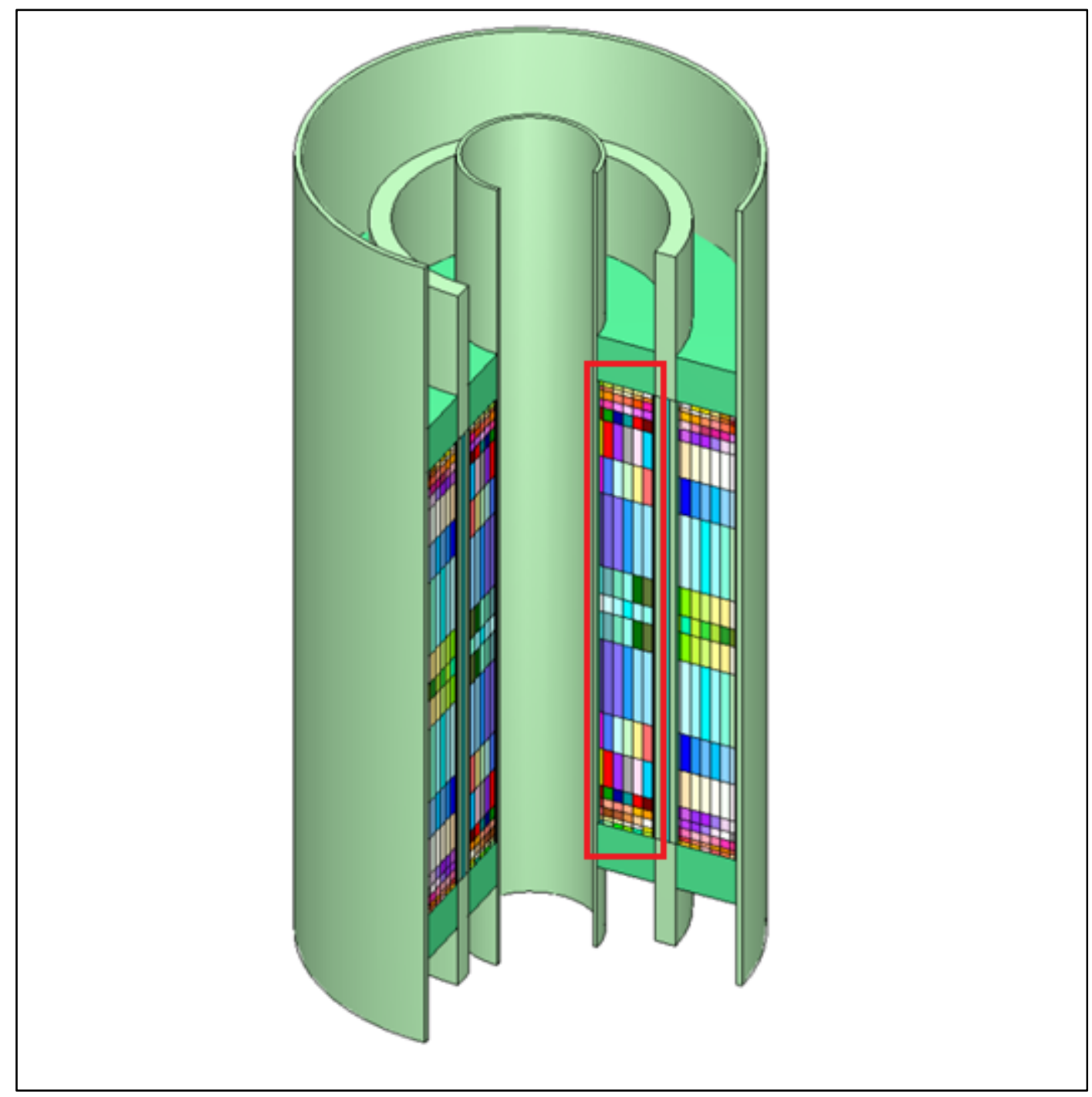

Fig. 5.7. MCNP Cells. The Inner Fuel Element cells are boxed in red.

For this thermal-hydraulic study, however, it is more convenient to restrict the nuclear power generation to the fuel meat alone. While not strictly physical it does produce a conservative temperature estimate as an equal power output is being produced in a smaller volume, thus raising the local power densities in the fuel. To make the conversion from power-per-cell-volume to power-per-fuel meatvolume, $P_{f u e l}^{\prime \prime \prime}$, the MCNP power density output can be divided by the ratio of a cell's fuel meat volumeto-total volume, $V_{\text {ratio }}$. Given that width and height are equal for both fuel meat and total cell volume, it is essentially a ratio of the average fuel meat thickness in each cell normalized against the cell's thickness. The result is thus the fuel meat's thickness profile as a function of radius. 


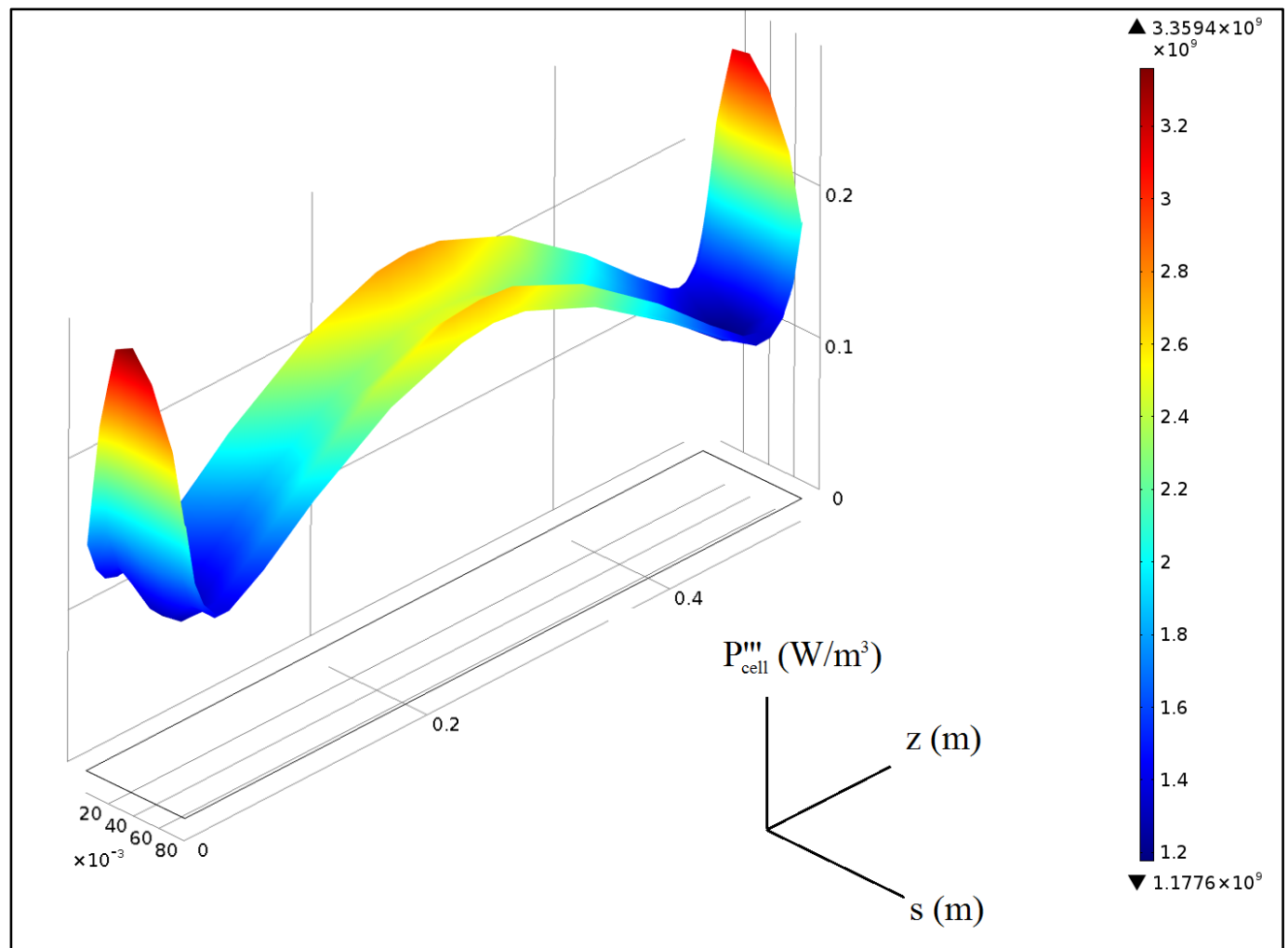

Fig. 5.8. MCNP Power Density Profile. The direction of flow is in the $+z$-direction.

The thickness data is imported directly into COMSOL alongside the power-per-cell-volume nuclear power generation density. The final power-per-fuel meat-volume is calculated by dividing the MCNP power density output by the volume ratio of each cell as given in Eqn. (5.1)

$$
P_{\text {fuel }}^{\prime \prime \prime \prime}{ }^{i}=c_{1}^{i} \times \frac{P_{\text {cell }}^{\prime \prime \prime}{ }^{i}}{V_{\text {ratio }}^{i}}
$$

where $i$ is an index denoting cell-specific values and the coefficient $c_{l}$ is a correction factor that can be used to manually set the magnitude of the distribution in order to maintain the relation

$$
P_{\text {plate }}=\sum_{i=1}^{I} P_{\text {fuel }}^{\prime \prime \prime}{ }^{i} \times V_{\text {fuel }}^{i}
$$

where $I$ is the total number of MCNP cells in a single plate and the total power output, $P_{\text {plate }}$, is known to be $217.72 \mathrm{~kW}$. The total power output can be determined independently according to

$$
P_{\text {plate }}=P_{\text {core }} \times f_{\text {IFE }} / n_{\text {IFE }}
$$


where $P_{\text {core }}$ is the total power output of the core, $100 \mathrm{MW}, f_{I F E}$ is the fraction of the total power output produced in the inner fuel element, $37.23 \%$, and $n_{I F E}$ is the total number of inner fuel element plates, 171. The final power density profile that is input into COMSOL is shown in Fig. 5.9.

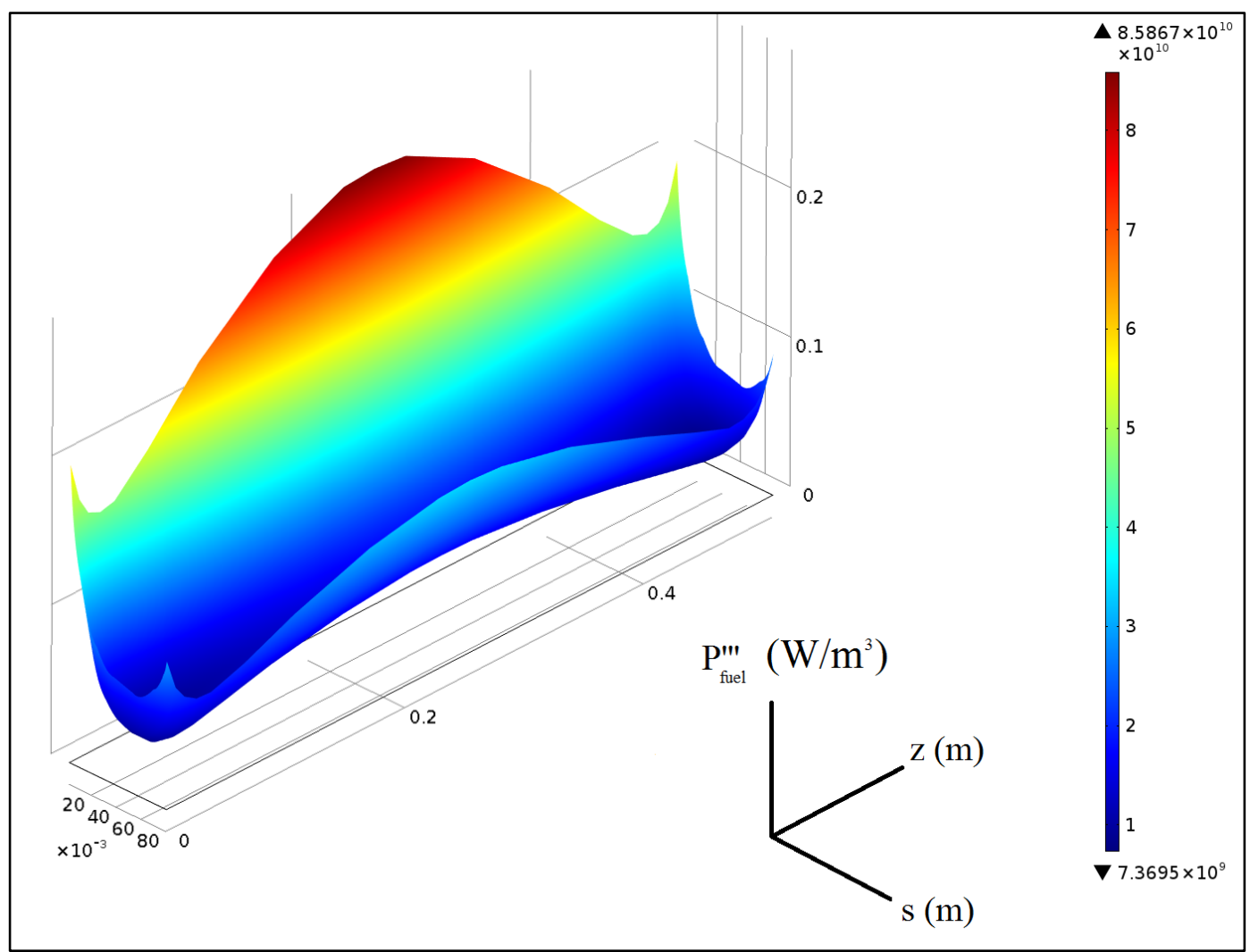

Fig. 5.9. COMSOL Power Density Profile. The direction of flow is in the $+z$-direction.

\section{Physics}

Energy transport occurs through three methods in the 3D model: heat transfer in the solid domains, heat transfer in the coolant domain, and turbulent mass transport in the coolant domain. Heat transfer in the solid domains is governed by the steady-state heat equation ${ }^{68}$

$$
\nabla \cdot(\lambda \nabla T)+Q=0
$$

where thermal conductivity, $\lambda$, is a function of $T$ and thus dependent on position and $Q$ is volumetric heat generation. The total volumetric heat generation, $Q_{t o t}$, is defined such that all of the power generated in the fuel plate is assumed to be in form of heat energy 


$$
Q_{\text {tot }}=P_{\text {plate }}^{\prime \prime \prime}
$$

It is important to note that the involute coordinate system $(s, t, z)$ was developed only to aid with the specification of model inputs and the analysis of results. The computational scheme still operates in a Cartesian coordinate system $(x, y, z)$. The gradient thus has the familiar form

$$
\nabla=\frac{\partial}{\partial x} \hat{i}+\frac{\partial}{\partial y} \hat{j}+\frac{\partial}{\partial z} \hat{k}
$$

The volumetric heat generation term $Q$ in Eqn. (5.4) is present only in the fuel meat. In the surrounding aluminum fuel plate and side plates $Q$ equals zero causing Eqn. (5.4) to reduce to Fourier's law, giving rise to linear heat flow according to

$$
-\lambda \nabla T=q^{\prime \prime}
$$

where $q^{\prime \prime}$ represents the heat flux vector with components $q_{x}^{\prime \prime}, q_{y}^{\prime \prime}$, and $q_{z}^{\prime \prime}$. The solid domains experience three types of boundaries: thermally insulated, convective, and periodic. Thermally insulated boundaries are applied to the inner surface of the inner side plate and the outer surface of the outer side plate - the inner and outer circumferences of the model, respectively. The thermally insulated boundaries satisfy the Neumann-type constraint that the temperature gradient be zero such that

$$
\hat{\boldsymbol{n}} \cdot(-\lambda \nabla T)=q_{n}^{\prime \prime}=0
$$

where $\hat{n}$ represents the local boundary normal vector. The convective boundaries ensure continuity of temperature and heat flux across the solid-liquid interface. The periodic boundaries, applied to the concave and convex faces of the side plates, enforce the same conditions.

Mass transport in the coolant domain is governed by the Reynolds-Averaged Navier-Stokes (RANS) equations with the $k-\varepsilon$ turbulence model chosen to provide closure. In the most recent COMSOL release at the onset of this project, version 4.3.A, the only available turbulence models were $k-\varepsilon, k-\omega$, and LowReynolds Number (LRN) $k-\varepsilon$. The $k-\varepsilon$ model was selected through the process of reduction. The models were considered on a two-tiered scheme with the two-equation $k-\varepsilon$ and $k$ - $\omega$ models being on a similar level of complexity and the LRN $k-\varepsilon$ model adding an additional level.

The LRN $k-\varepsilon$ model's added complexity comes from the fact that it explicitly models the fluid domain in its entirety, including all the way up to wall boundaries. The two other models, by contrast, utilize wall functions to simplify the already complex boundary layer region. Use of the LRN $k-\varepsilon$ model requires considerably more boundary-layer mesh elements which in turn gives rise to added computational cost, 
stability issues, and meshing difficulties. Given the desire to produce as efficient a model as possible with the coupled model, the LRN $k-\varepsilon$ turbulence model was considered too expensive for use.

The two remaining turbulence models were both tested on simplified proof-of-concept models and the completed 3D model. In the simplified models the $k$ - $\omega$ model displayed a marginally faster convergence rate than the $k-\varepsilon$ model. In the $3 \mathrm{D}$ model, however, the $k$ - $\omega$ suffered from stability issues resultant of the high-aspect ratio mesh elements in the narrow coolant channel. These effects were especially pronounced in corners between the fuel plate and side plates where inter-element mesh structures often became mangled. While this problem can be overcome through the use of more advanced meshing techniques not incorporated in either model, the $k-\varepsilon$ model proved the most stable and robust of the available turbulence models.

Both models consider variable-property flow in which coolant properties are given as functions of temperature. In order to model such a flow, the use of the semi-compressible N-S equations is required. Favre mass-averaging is used to decompose flow variables in lieu of the traditional Reynolds timeaveraging which is insufficient for compressible flow. Reynolds time-averaging is still used, not only to define the Favre averages, but also in the manipulation of the N-S equations. The final set of equations, therefore, will still be referred to as the RANS equations.

In Favre averaging flow variables, $\phi$, are broken down into mass-averaged, $\widetilde{\phi}$, and fluctuating components, $\phi^{\prime \prime}$, such that

$$
\phi=\widetilde{\phi}+\phi^{\prime \prime}
$$

where the mass-averaged term is given by

$$
\widetilde{\phi}=\frac{\overline{\rho \phi}}{\bar{\rho}}
$$

where overbars indicate time-averaged quantities. Multiplying Eqn. (5.9) through by $\rho$ and timeaveraging each term yields

$$
\overline{\rho \phi}=\bar{\rho} \widetilde{\phi}+\overline{\rho \phi^{\prime \prime}}
$$

The mass-averaged flow variable is unchanged since it is already composed entirely of time-averaged components and the time average of a time-averaged term does not change. Applying the relation in Eqn. (5.10) to Eqn. (5.11) dictates that the left-hand side equals the first term on the right-hand side such that 


$$
\overline{\rho \phi^{\prime \prime}}=0
$$

The RANS equations can be derived by beginning with the steady-state, compressible N-S equations for divergence-free flow

$$
\begin{gathered}
\nabla \cdot(\rho \boldsymbol{u})=0 \\
\rho \boldsymbol{u} \cdot \nabla \boldsymbol{u}=-\nabla p+\nabla \cdot \mu\left[\nabla \boldsymbol{u}+(\nabla \boldsymbol{u})^{T}\right]-\nabla \cdot\left[\frac{2}{3} \mu \nabla \cdot \boldsymbol{u}\right]
\end{gathered}
$$

where $\boldsymbol{u}$ is the velocity vector, the first bracketed term in the momentum equation, is the viscous stress tensor, a superscript $T$ denotes the transpose of a matrix, and the second bracketed term of the momentum equation accounts for compressibility effects. Inspection of the compressible momentum equation shows that it is identical to the incompressible momentum equation except for the addition of the final term on the right-hand side of Eqn. (5.13b). The left-hand side of Eqn. (5.13b) can be rewritten in conservation form to produce

$$
\rho \boldsymbol{u} \cdot \nabla \boldsymbol{u}=\nabla \cdot\left(\rho \boldsymbol{u} \boldsymbol{u}^{T}\right)-\boldsymbol{u}^{T}[\nabla \cdot(\rho \boldsymbol{u})]
$$

where the final term in Eqn. (5.14) is zero given the continuity condition in Eqn. (5.13a). The momentum equation then becomes

$$
\nabla \cdot\left(\rho \boldsymbol{u} \boldsymbol{u}^{T}\right)=-\nabla p+\nabla \cdot \mu\left[\nabla \boldsymbol{u}+(\nabla \boldsymbol{u})^{T}\right]-\nabla \cdot\left[\frac{2}{3} \mu \nabla \cdot \boldsymbol{u}\right]
$$

The velocity vector can be rewritten via Favre averaging in decomposed form

$$
\boldsymbol{u}=\tilde{\boldsymbol{u}}+\boldsymbol{u}^{\prime \prime}
$$

The pressure can be similarly decomposed. Substituting these decompositions into Eqn. (5.15) produces

$$
\nabla \cdot\left[\rho\left(\widetilde{\boldsymbol{u}}+\boldsymbol{u}^{\prime \prime}\right)\left(\widetilde{\boldsymbol{u}}^{T}+\boldsymbol{u}^{T^{\prime \prime}}\right)\right]=-\nabla\left(\widetilde{p}+p^{\prime \prime}\right)+\nabla \cdot \mu\left[\nabla\left(\widetilde{\boldsymbol{u}}+\boldsymbol{u}^{\prime \prime}\right)+\left(\nabla\left(\widetilde{\boldsymbol{u}}+\boldsymbol{u}^{\prime \prime}\right)\right)^{T}\right]-\nabla \cdot\left[\frac{2}{3} \mu \nabla \cdot\left(\widetilde{\boldsymbol{u}}+\boldsymbol{u}^{\prime \prime}\right)\right]
$$

where the term on the left-hand side when multiplied out becomes

$$
\nabla \cdot\left[\rho\left(\widetilde{\boldsymbol{u}} \widetilde{\boldsymbol{u}}^{T}+\widetilde{\boldsymbol{u}}^{T} \boldsymbol{u}^{\prime \prime}+\widetilde{\boldsymbol{u}} \boldsymbol{u}^{T^{\prime \prime}}+\boldsymbol{u}^{\prime \prime} \boldsymbol{u}^{T}\right)\right]=[\ldots]
$$


The N-S equations are then time-averaged. As stated before, the time average of a mass-averaged component is itself and the time average of a fluctuating component times the time-averaged density is zero. This extends to terms on the right-hand side of the momentum equation multiplied by $\mu$ which is dependent on density. The RANS momentum equation then reduces to

$$
\nabla \cdot\left(\bar{\rho} \widetilde{\boldsymbol{u}} \widetilde{\boldsymbol{u}}^{T}+\overline{\rho \boldsymbol{u}^{\prime \prime} \boldsymbol{u}^{T}}\right)=-\nabla \widetilde{p}+\nabla \cdot \bar{\mu}\left[\nabla \widetilde{\boldsymbol{u}}+(\nabla \widetilde{\boldsymbol{u}})^{T}\right]-\nabla \cdot\left[\frac{2}{3} \bar{\mu} \nabla \cdot \widetilde{\boldsymbol{u}}\right]
$$

where the time average of the product of density, the fluctuating velocity component, and its transpose is not necessarily zero. The viscous stress tensor and the specific Favre-averaged Reynolds stress tensor can be written, respectively, as

$$
\overline{\boldsymbol{\tau}}_{S}=\bar{\mu}\left[\nabla \widetilde{\boldsymbol{u}}+(\nabla \widetilde{\boldsymbol{u}})^{T}-\frac{2}{3} \nabla \cdot \widetilde{\boldsymbol{u}}\right]
$$

and

$$
\bar{\rho} \tau_{R}=-\overline{\rho u^{\prime \prime} \boldsymbol{u}^{T \prime}} .
$$

The final compressible RANS equations become

$$
\begin{gathered}
\nabla \cdot(\bar{\rho} \widetilde{\boldsymbol{u}})=0 \\
\bar{\rho} \widetilde{\boldsymbol{u}} \cdot \nabla \widetilde{\boldsymbol{u}}=-\nabla \widetilde{p}+\nabla \cdot\left(\overline{\boldsymbol{\tau}}_{S}+\bar{\rho} \tau_{R}\right)
\end{gathered}
$$

For three-dimensional Cartesian flow there are ten unknowns in all-four flow variables (pressure and the three components of the velocity vector) and six terms from the specific Reynolds stress tensor. There are only six unknowns in the specific Reynolds stress tensor, versus the possible nine, due to its inherent symmetry given that the magnitude of $u_{i} u_{j}$ equals $u_{j} u_{i}$ for all $i$ and $j$.

The Reynolds-stress equation can be derived through further manipulation of the RANS momentum equation via averaging of the form

$$
\overline{\boldsymbol{u}^{\prime \prime} \mathrm{N}(\boldsymbol{u})+\left(\boldsymbol{u}^{\prime \prime} \mathrm{N}(\boldsymbol{u})\right)^{T}}=0
$$

where $\mathrm{N}$ is the Navier-Stokes operator combining every element of Eqn.(5.22b) on the left-hand side. From here the two-equation $k-\varepsilon$ model is chosen to provide closure for the RANS equations. Specific turbulent kinetic energy, $k$, is first defined as one-half the sum of the three normal Reynolds stresses 


$$
k=\frac{1}{2}\left(\overline{u_{11}^{\prime 2}}+\overline{u_{22}^{\prime 2}}+\overline{u_{33}^{\prime 2}}\right)
$$

This is proportional to the trace of the Reynolds stress tensor

$$
k=-\frac{1}{2} \cdot \operatorname{tr}\left(\tau_{R}\right)=-\frac{1}{2} \sum_{i=1}^{3} \overline{u_{i i}^{\prime 2}} .
$$

The specific Reynolds stress tensor is then defined using $k$ according to

$$
\bar{\rho} \tau_{R}=\mu_{T}\left(\nabla \tilde{\boldsymbol{u}}+(\nabla \widetilde{\boldsymbol{u}})^{T}-\frac{2}{3} \nabla \cdot \tilde{\boldsymbol{u}}\right)-\frac{2}{3} \bar{\rho} k=\frac{\mu_{T}}{\bar{\mu}} \overline{\boldsymbol{\tau}}_{S}-\frac{2}{3} \bar{\rho} k
$$

where the dynamic eddy viscosity, $\mu_{T}$, is given by

$$
\mu_{T}=\bar{\rho} C_{\mu} \frac{k^{2}}{\varepsilon}
$$

The stress terms in Eqn. (5.22b) are now fully defined in terms of $\boldsymbol{u}$ and $k$. A transport equation is thus needed to solve $k$ in order to complete the equation set. It is found by taking the trace of the Reynoldsstress equation

$$
\bar{\rho} \tilde{\boldsymbol{u}} \cdot \nabla k=\nabla \cdot\left[\left(\bar{\mu}+\frac{\mu_{T}}{\sigma_{k}}\right) \nabla k\right]+\mathrm{P}_{k}-\bar{\rho} \varepsilon
$$

where the turbulence production term, $\mathrm{P}_{\mathrm{k}}$, is given by

$$
\mathrm{P}_{k}=\bar{\rho} \tau_{R} \nabla \tilde{\boldsymbol{u}}
$$

Again, another flow variable, the dissipation rate of turbulent energy, $\varepsilon$, is introduced in the course of solving for $k$. Its transport equation is given by

$$
\bar{\rho} \widetilde{\boldsymbol{u}} \cdot \nabla \varepsilon=\nabla \cdot\left[\left(\bar{\mu}+\frac{\mu_{T}}{\sigma_{\varepsilon}}\right) \nabla \varepsilon\right]+C_{\varepsilon 1} \frac{\varepsilon}{k} \mathrm{P}_{k}-C_{\varepsilon 2} \bar{\rho} \frac{\varepsilon^{2}}{k}
$$

Dimensionless constants for both turbulent transport equations are given in Table 5.4. ${ }^{69}$ 


\begin{tabular}{|c|c|}
\hline $\begin{array}{c}\text { Table 5.4: } \\
\text { Constants }\end{array}$ \\
\hline$C_{\mu}$ & 0.09 \\
\hline$C_{\varepsilon 1}$ & 1.44 \\
\hline$C_{\varepsilon 2}$ & 1.92 \\
\hline$\sigma_{k}$ & 1.00 \\
\hline$\sigma_{\varepsilon}$ & 1.30 \\
\hline
\end{tabular}

The turbulent Prandlt number, $\operatorname{Pr}_{T}$, which provides the ratio between momentum eddy diffusivity and thermal eddy diffusivity, is calculated using the Kays-Crawford method. ${ }^{70}$ The Kays-Crawford formulation was used due to its general applicability to wall-bounded turbulent flows. The formulation is

$$
\operatorname{Pr}_{T}=\left[\frac{1}{2 \operatorname{Pr}_{T \infty}}+\frac{0.3}{\sqrt{\operatorname{Pr}_{T \infty}}} \frac{c_{p} \mu_{T}}{\lambda}-\left(0.3 \frac{c_{p} \mu_{T}}{\lambda}\right)^{2}\left(1-e^{-\frac{\lambda}{0.3 c_{p} \mu_{T} \sqrt{\mathrm{Pr}_{T \infty}}}}\right)\right]^{-1}
$$

where the turbulent Prandtl number at a theoretical point at an infinite distance perpendicular from the wall, $\operatorname{Pr}_{T_{\infty}}$ is 0.85 . Heat transfer is governed by the heat equation for translating media:

$$
\bar{\rho} c_{p} \tilde{\boldsymbol{u}} \cdot \nabla T=\nabla \cdot(\lambda \nabla T)
$$

At the inlet, Dirichlet boundary conditions are imposed for temperature and velocity based on the model inputs in Table 5.3. Dirichlet conditions are also imposed for specific turbulent kinetic energy and dissipation rate. They are

$$
k_{\text {in }}=\frac{3}{2}\left(\left\|\boldsymbol{u}_{\text {in }}\right\| I_{T}\right)^{2}
$$

and

$$
\varepsilon_{i n}=C_{\mu}^{3 / 4} \frac{k_{i n}^{3 / 2}}{L_{T}}
$$

where $I_{T}$ is the turbulence intensity and $L_{T}$ is the turbulent length scale. Typical values for $I_{T}$ in fully turbulent flows typically vary between $5 \%$ and $10 \% .{ }^{69}$ Five percent was chosen for the 3D model. $L_{T}$ was set to $7 \%$ of the hydraulic diameter, $D_{H}$, which is a standard value for fully developed pipe flow. 
A Dirichlet condition was specified for pressure on the outlet according to the value in Table 5.3. Homogeneous Neumann conditions were imposed for specific turbulent kinetic energy, dissipation rate, and viscous stress such that

$$
\begin{gathered}
\hat{\boldsymbol{n}} \cdot \nabla k=0 \\
\hat{\boldsymbol{n}} \cdot \nabla \varepsilon=0 \\
\hat{\boldsymbol{n}} \cdot\left\{\left(\bar{\mu}+\mu_{T}\right)\left[\nabla \widetilde{\boldsymbol{u}}+(\nabla \widetilde{\boldsymbol{u}})^{T}-\frac{2}{3} \nabla \cdot \widetilde{\boldsymbol{u}}\right]-\frac{2}{3} \bar{\rho} k\right\}=0 .
\end{gathered}
$$

The inward facing coolant boundary that is the result of the difference in lengths between the inner and outer side plates is bounded using an open condition. The open boundary condition can function as either a pseudo-inlet or pseudo-outlet boundary condition depending on the sign of $\boldsymbol{u} \cdot \hat{\boldsymbol{n}}$. Given the open boundary's location adjacent to the coolant outlet at the bottom of the model, in this instance it functions purely under outlet condition restraints. The open boundary condition imposes Eqns. (5.35)-(5.37) along with a homogeneous Neumann condition for temperature

$$
\hat{\boldsymbol{n}} \cdot \nabla T=0
$$

Both the coolant outlet and the bottom faces of the side plates serve as heat outflows in which Eqn. (5.8) is imposed to ensure that the normal temperature gradient is zero. The open coolant boundary also serves as a heat outflow, with the condition of zero heat flux inherently specified.

The periodic coolant boundaries impose the same continuity conditions on temperature and its gradient as the periodic boundaries in the side plates. Additional continuity conditions are specified for coolant velocity, pressure, specific turbulent kinetic energy, and dissipation rate.

The final boundary type in the coolant domain is the solid, no-slip, impermeable wall. The $k-\varepsilon$ turbulence model utilizes wall functions to model near-wall boundary layer flow. This creates a gap of thickness $\delta_{w}$ between the physical wall and the edge of the computational fluid domain across which the fluid flow is not explicitly modeled. This wall-offset distance is calculated according to

$$
\delta_{w}^{+}=\frac{\bar{\rho} u_{\tau} \delta_{w}}{\bar{\mu}}
$$

where $\delta_{w}^{+}$is the dimensionless wall-offset distance and the shear velocity, $u_{\tau}$, is provided by 


$$
u_{\tau}=C_{\mu}^{1 / 4} k^{1 / 2}
$$

The wall-offset distance is calculated as half the height of the local wall-adjacent boundary mesh element or that value which produces a $\delta_{w}^{+}$of 11.06 , whichever is greater. A $\delta_{w}^{+}$of 11.06 corresponds to the dimensionless distance from the wall, $y_{c}^{+}$, where the linear velocity profile relationship of the nearwall viscous sublayer intersects the logarithmic relationship of the inner log-law layer. This intersection is shown in Fig. 5.10. In reality, the velocity profile does not adhere to either relationship all the way up to the point of intersection but connects smoothly between the two across a buffer layer, as demonstrated by the Spalding approximation.

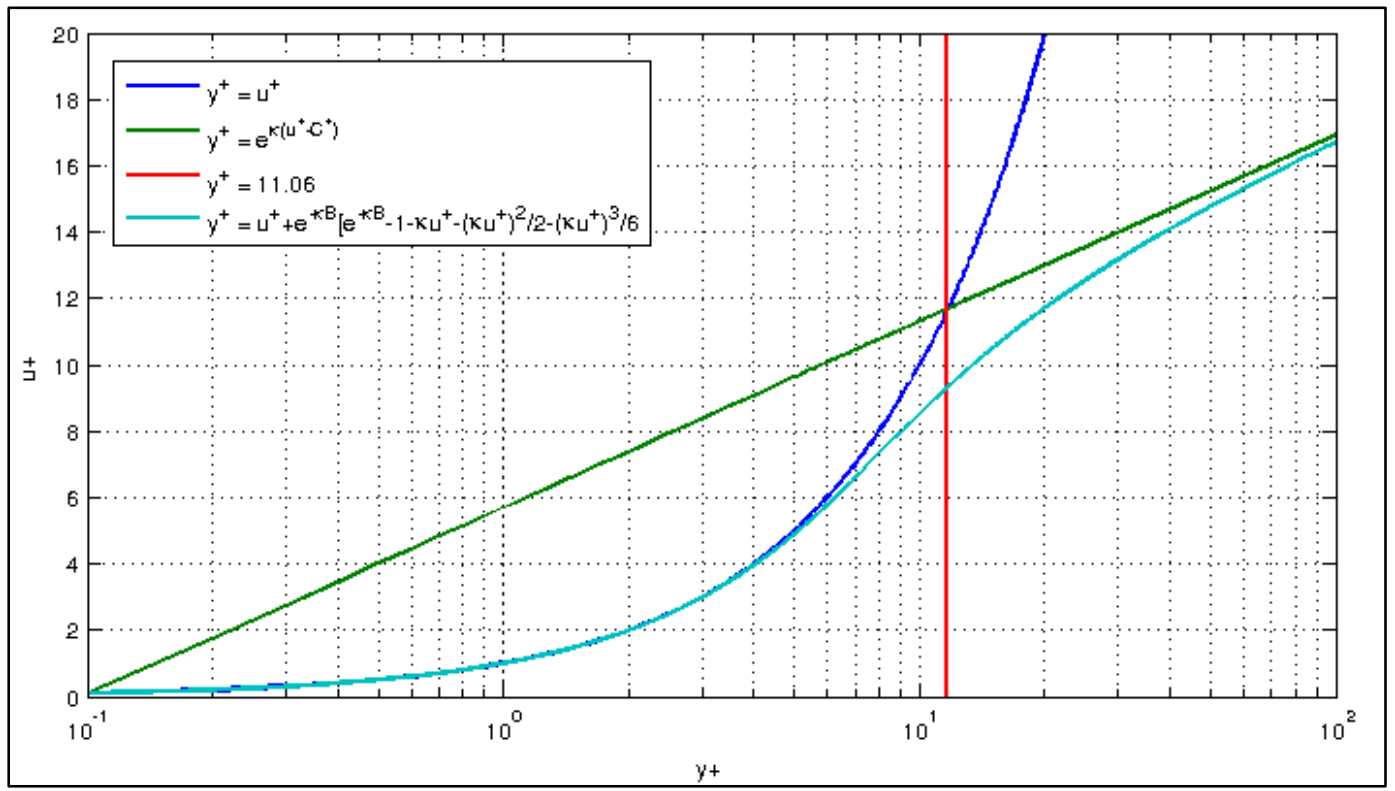

Fig. 5.10. Law of the Wall. The linear relation for $u^{+}$, in blue, is valid to the left of the intersection point in the viscous sublayer. The logarithmic relation, in green, is valid to the right in the inner log-law layer. The smooth Spalding approximation, in teal, is valid throughout. ${ }^{71}$ The von Kármán constant, $\kappa$, equals 0.41 . $B$ equals $\sim 5.5$.

The velocity profile across the computational gap $\delta_{w}^{+}$is modeled using the linear relationship of the viscous sublayer according to ${ }^{72}$

$$
u_{\tan }\left(y_{c}\right)=\frac{u_{\tan }\left(\delta_{w}\right)}{\delta_{w}} y_{c}, \quad 0 \leq y_{c} \leq \delta_{w}
$$


where $u_{\text {tan }}$ is the velocity magnitude tangent to the wall. A no-penetration Dirichlet condition is imposed on the normal velocity component along the $\delta_{w}^{+}$border to ensure that the flow across the computational gap is purely tangential

$$
\hat{\boldsymbol{n}} \cdot \tilde{\boldsymbol{u}}=0
$$

A homogenous Neumann condition is also specified for $k$. A Dirichlet condition is imposed for $\varepsilon$ of the form

$$
\varepsilon_{\delta_{w}}=C_{\mu}^{3 / 4} \frac{k_{\delta_{w}}^{3 / 2}}{\kappa \delta_{w}}
$$

The shear stress on the $\delta_{w}^{+}$border is constrained by

$$
\hat{\boldsymbol{n}} \cdot\left\{\left(\mu+\mu_{T}\right)\left[\nabla \widetilde{\boldsymbol{u}}+(\nabla \widetilde{\boldsymbol{u}})^{T}-\frac{2}{3} \nabla \cdot \widetilde{\boldsymbol{u}}\right]-\frac{2}{3} \bar{\rho} k\right\}=-\bar{\rho} \frac{u_{\tau}}{\delta_{w}^{+}}[\widetilde{\boldsymbol{u}}-(\widetilde{\boldsymbol{u}} \cdot \hat{\boldsymbol{n}}) \hat{\boldsymbol{n}}]
$$

Lastly, the heat flux across the computational gap, $q_{w f}$, is given by

$$
q_{w f}^{\prime \prime}=\frac{\bar{\rho} c_{p} C_{\mu}^{1 / 4} k^{1 / 2}\left(T_{w}-T_{f}\right)}{T^{+}}
$$

where the dimensionless temperature, $T^{+}$, is determined as a function of $\delta_{w}^{+}$

$$
T=\left\{\begin{array}{cl}
\operatorname{Pr} \delta_{w}^{+}, & \text {if } \delta_{w}^{+} \leq \delta_{1}^{+} \\
15 \operatorname{Pr}^{2 / 3}-\frac{500}{\delta_{2}^{+}}, & \text {if } \delta_{1}^{+} \leq \delta_{w}^{+}<\delta_{2}^{+} \\
\frac{\operatorname{Pr}}{\kappa} \ln \left(\delta_{w}^{+}\right)+15 \operatorname{Pr}^{2 / 3}-\frac{\operatorname{Pr}_{T}}{2 \kappa}\left[1+\ln \left(1000 \frac{\kappa}{\operatorname{Pr}_{T}}\right)\right], & \text { if } \quad \delta_{2}^{+} \leq \delta_{w}^{+}
\end{array}\right.
$$

where

$$
\begin{gathered}
\delta_{1}^{+}=10 \operatorname{Pr}^{-1 / 3}, \\
\delta_{2}^{+}=\sqrt{1000 \frac{\kappa}{\operatorname{Pr}_{T}}},
\end{gathered}
$$

and 


$$
\operatorname{Pr}=\frac{c_{p} \mu}{\lambda}
$$

\section{Meshing}

The axial consistency of the 3D model makes meshing a relatively straightforward affair. Many geometry components can be meshed by mapping a 2D surface in the $s$ - $t$ plane and extruding down their axial lengths. This extrusion process was modeled after the MCNP cell distribution. Tests showed that, as a minimum, the fuel meat, fuel plate, and adjacent coolant should be mapped with an axial mesh distribution at least equal to the MCNP cell distribution. The MCNP axial cell distribution is shown in Table 5.5. The MCNP distribution is only defined for the length of the fuel meat. It is symmetric about the axial midplane of the fuel meat with tighter cell spacings in the center and at the ends corresponding to areas with the greatest heat generation.

This MCNP distribution was continued into the adjacent coolant and side plates. For geometries upand downstream of the fueled region, axial distributions were devised that were comparable to the MCNP distribution. In order to increase the smoothness of solutions, the MCNP mesh distribution was doubled in the final 3D model. Both axial distributions are shown in Fig. 5.11.

All of the distinct mesh domains are shown via diagram in Fig. 5.12. Mesh domains are color-coded according to the mesh type associated with them. The most predominant, and the most ideal, mesh type is the structured mesh which is applicable to every axially-consistent geometry component free of internal vacancies. The clad surrounding the fuel meat is the only axially-consistent geometry incompatible with the structured mesh type. Due to the hole in the geometry's center where the fuel meat sits, its $s$ - $t$ crosssection had to be mapped using a free-triangular mesh which was then extruded the same as the structured meshes. A cross-sectional cut-out showing the $s-t$ plane through the fuel meat, surrounding clad, inner side plate, and coolant is shown in Fig. 5.13. 


\begin{tabular}{|c|c|}
\hline \multicolumn{2}{|c|}{$\begin{array}{l}\text { Table 5.5: MCNP Axial } \\
\text { Cell Distribution }\end{array}$} \\
\hline $\begin{array}{l}\text { Cell Wall Axial } \\
\text { Position }(\mathrm{cm})\end{array}$ & Cell Height $(\mathrm{cm})$ \\
\hline 50.8 & 0.5 \\
\hline 50.3 & 0.5 \\
\hline 49.8 & 1 \\
\hline 48.8 & 1 \\
\hline 47.8 & 1.4 \\
\hline 46.4 & 4.2 \\
\hline 42.2 & 4.2 \\
\hline 38 & 8.4 \\
\hline 29.6 & 3.2 \\
\hline 26.4 & 2 \\
\hline 24.4 & 3.2 \\
\hline 21.2 & 8.4 \\
\hline 12.8 & 4.2 \\
\hline 8.6 & 4.2 \\
\hline 4.4 & 1.4 \\
\hline 3 & 1 \\
\hline 2 & 1 \\
\hline 1 & 0.5 \\
\hline 0.5 & 0.5 \\
\hline 0 & \\
\hline
\end{tabular}

The remaining geometries - the rounded leading edge of the fuel plate and the leading and trailing edge coolant caps-were meshed using free-tetrahedral meshes unstructured in all three dimensions. They are shown in Fig. 5.14. Lastly, a boundary-layer mesh was added to every wall boundary in the coolant domain as demonstrated in Fig. 5.15. 


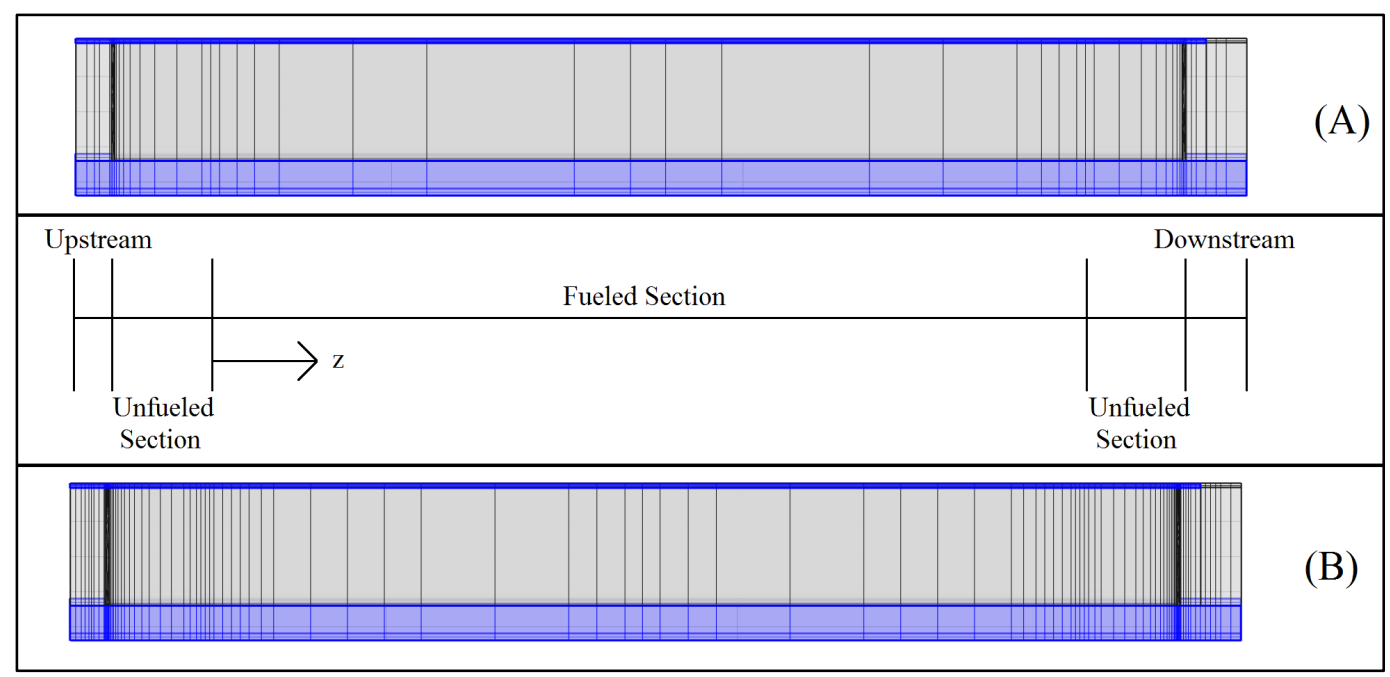

Fig. 5.11. 3D Axial Mesh Distributions. The MCNP axial mesh distribution is shown in (A) and the 2X MCNP distribution in (B). The side plates are highlighted in blue. The coolant channel is shown in grey. The fuel plate and fuel meat are obscured behind the coolant channel. Figure is not representative of final mesh as all spanwise (along the length of the involute curve) mesh distributions have been set to 1 for ease of viewing. The final mesh structure would not be discernible at this scale.

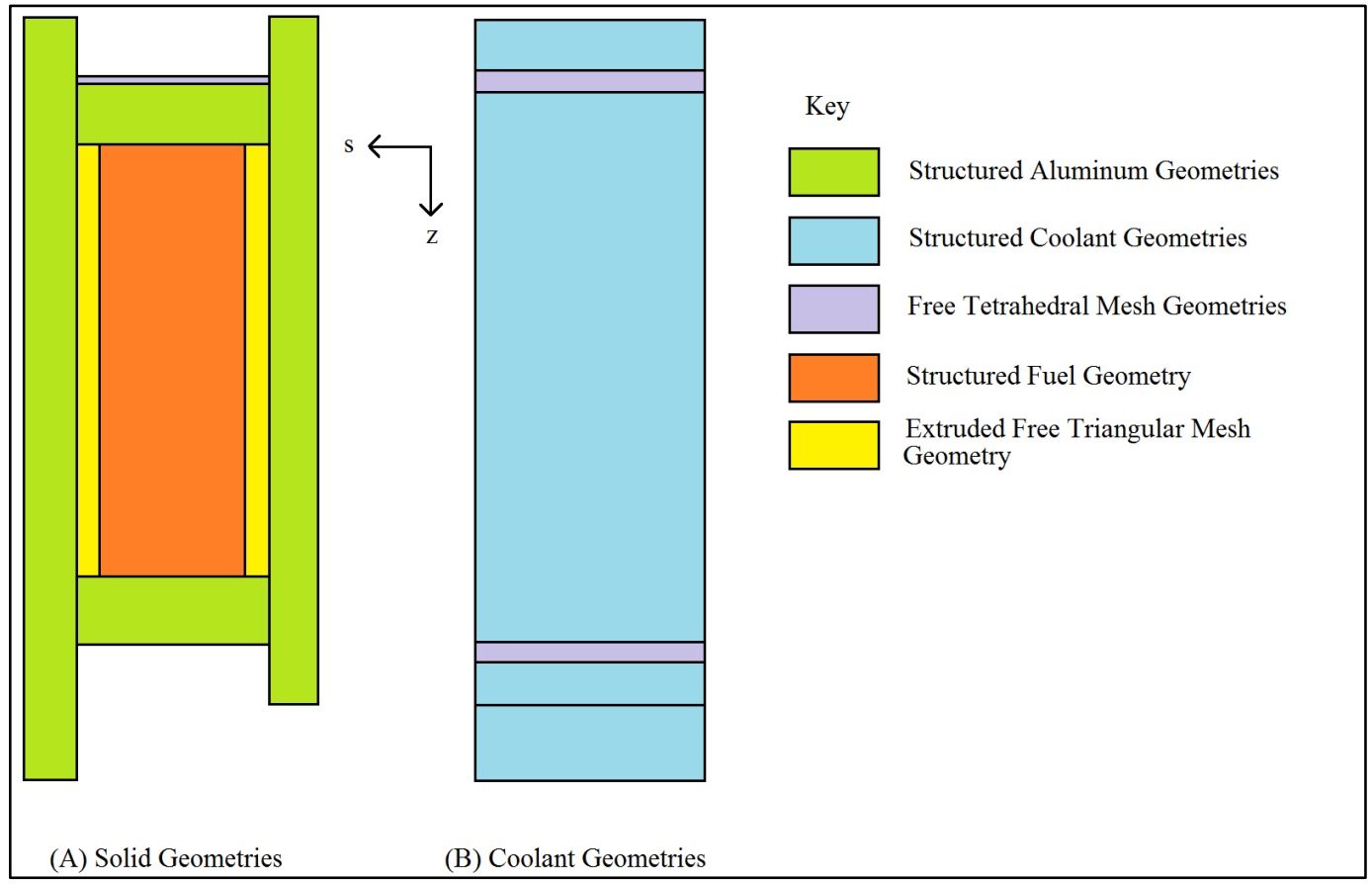

Fig. 5.12. Mesh Domains. Figure is not to scale. 


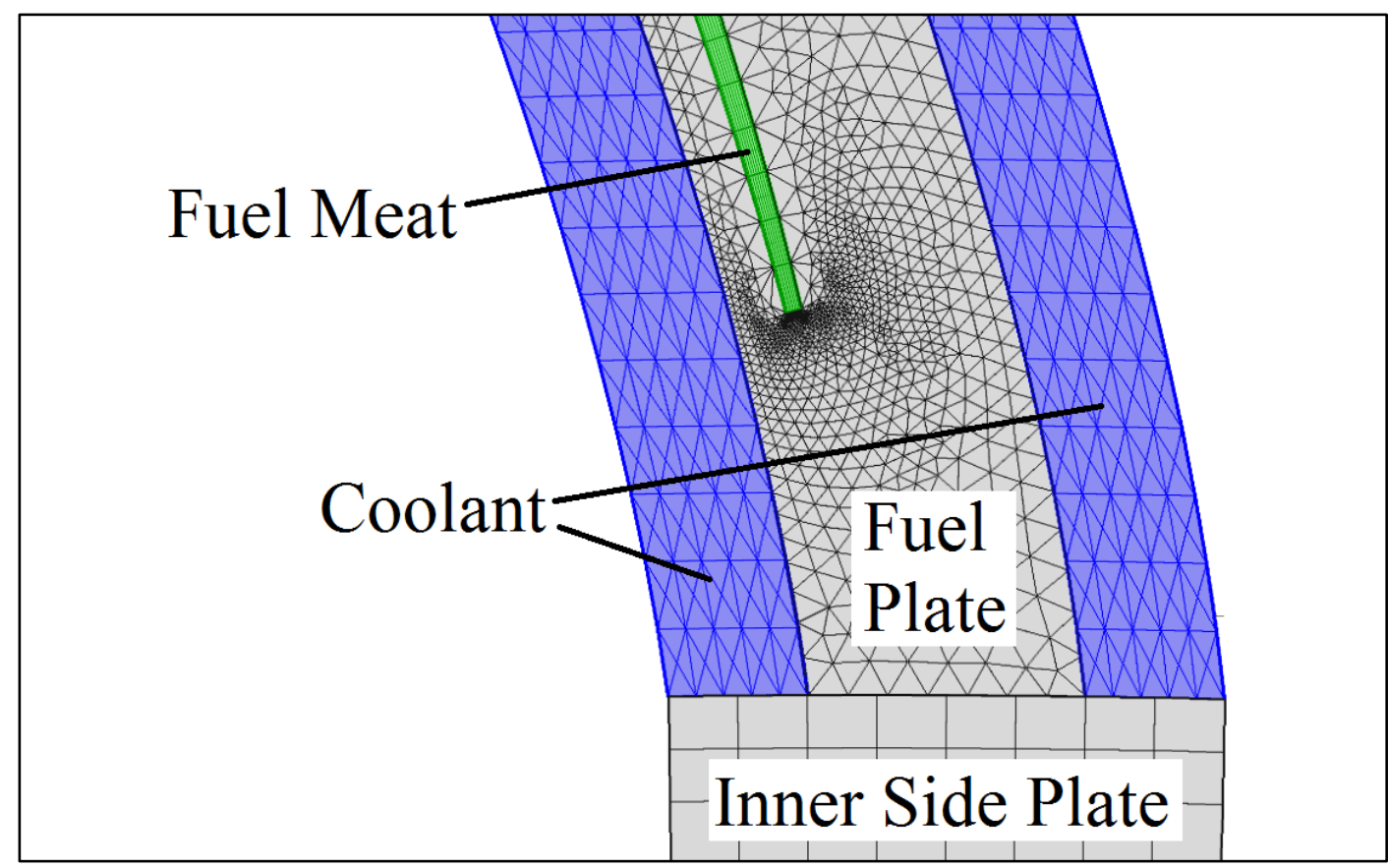

Fig. 5.13. Fueled Section Cross-Sectional Mesh. Structured quadrilateral mesh elements in the coolant region have been converted to triangular elements in order to facilitate meshing between axial coolant components.

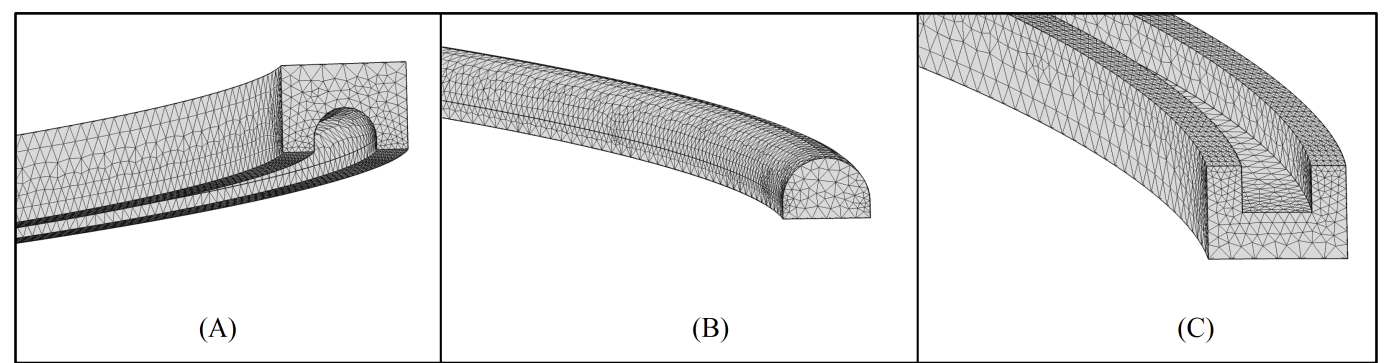

Fig. 5.14. Free-Tetrahedral Mesh Domains. The leading edge coolant cap geometry is shown in (A), the rounded leading edge clad in (B), and the trailing edge coolant cap in (C). 


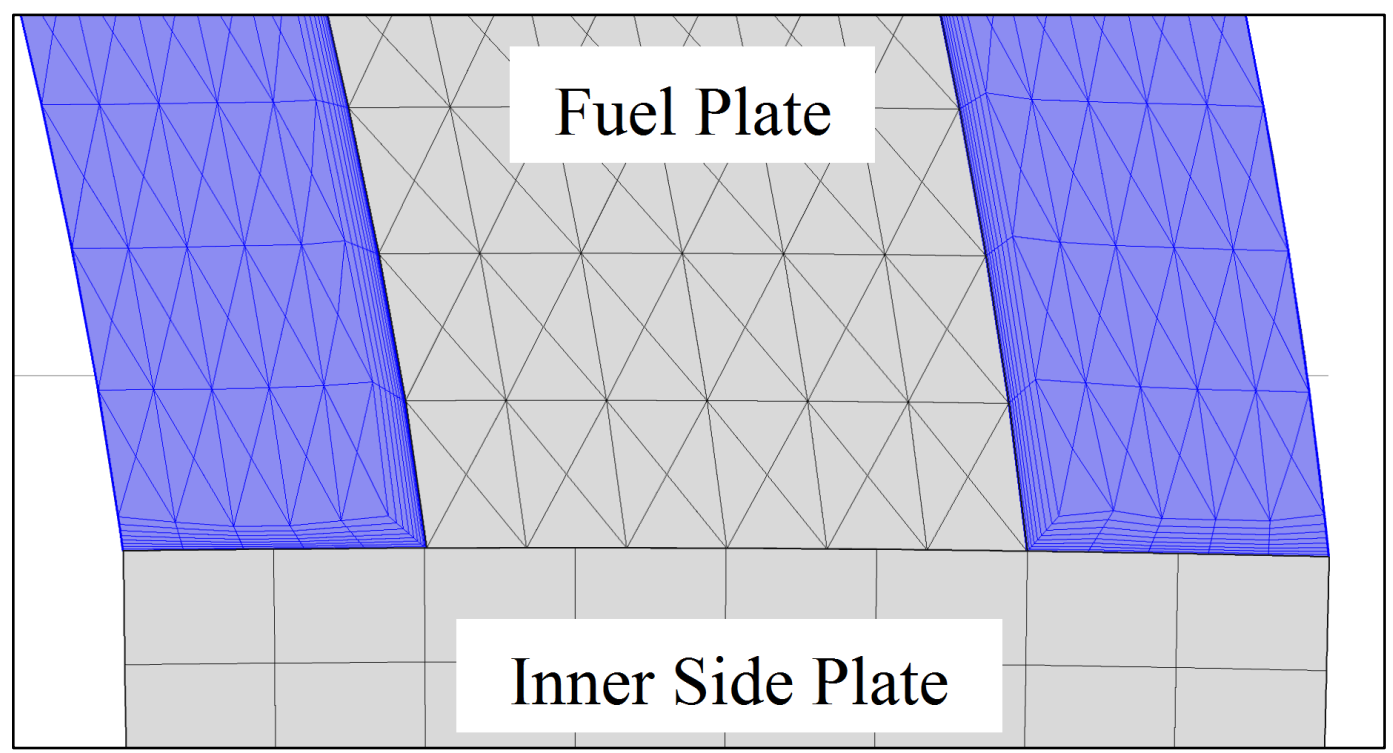

Fig. 5.15. Boundary-Layer Mesh Detail.

Given the use of the MCNP axial cell distribution, mesh convergence studies were performed by varying the resolution of the $s$ - $t$ plain face meshes only. Mesh densities were driven by the competing needs to produce a scale that was sufficiently small so as to capture all, or enough of, the underlying physics in a domain without adding undue complexity to the model. In the coolant domain this is most evident in the creation of the boundary layer and its effect on perceived near-wall viscous shear stresses. In the fuel meat, the problem arises in capturing the quadratic nature of the temperature profile resultant of internal heat generation. This quadratic nature can easily be demonstrated via solution of the 1D heat equation with heat generation

$$
-\lambda \frac{d^{2} T}{d x^{2}}=Q
$$

Integrating yields

$$
-\lambda \frac{d T}{d x}=Q x+D_{1}
$$

Integrating again and dividing by $\lambda$ produces a temperature profile with quadratic elements

$$
T(x)=-\frac{1}{\lambda}\left[\frac{Q x^{2}}{2}+D_{1} x+D_{2}\right] .
$$


The quadratic temperature profile is, obviously, most easily captured using quadratic basis functions. The continuity between the coolant and the solid domains in the 3D model, however, prevents multiple basis functions from being used, even in separate domains. In the COMSOL distribution utilized for thesis-related work, using quadratic basis functions to solve for temperature in the fuel meat means that they must also be used in the coolant. Such an approach proves computationally unfeasible as the model's degrees of freedom increases drastically due to the required resolution of the boundary-layer mesh. It is thus simpler and more expedient to increase the mesh resolution in the fuel meat and use linear elements throughout the model. 


\section{Coupled Model}

\section{A. Construction}

The three-dimensional fuel meat and fuel plate geometries used in the 3D model are also used in the coupled model. The coolant, however, is represented using a two-dimensional slice in the $t$ - $z$ plane as shown in Fig. 6.1. The axial heights of the entrance and exit coolant regions above and below the fuel plate, respectively, are the same as in the 3D model. The coolant domain is broken into three components for ease of meshing. The coolant domain's boundaries upstream and downstream of the fuel plate in the $t$-direction correspond to the midplane of the adjacent fuel plates.

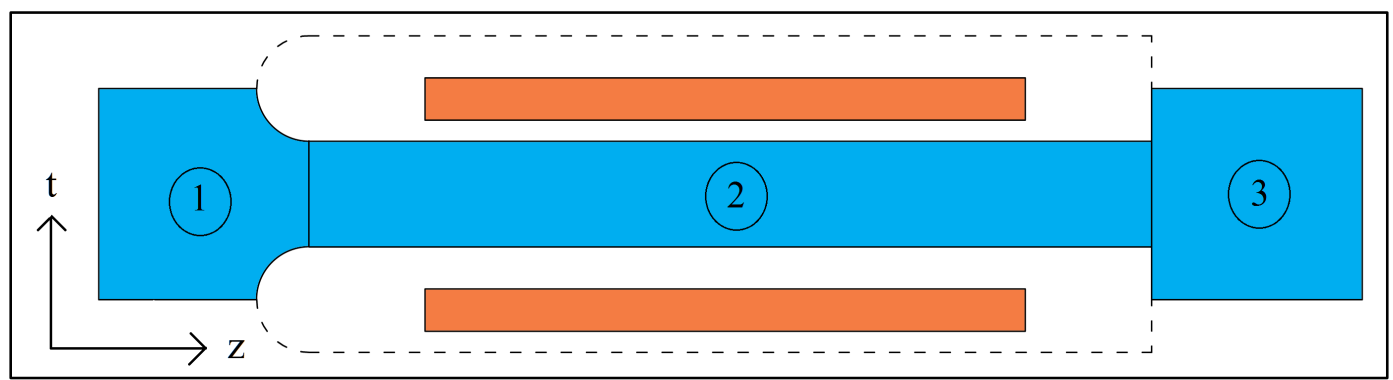

Fig. 6.1. Coupled Model Coolant Domain. The coolant slice is shown in blue. The obstructions created by the adjacent fuel plates in the coolant channel are marked with dotted lines. Note, however, that the fuel plates are not included in the 2D geometry. The approximate position of the fuel meat within each fuel plate is shown in orange. Flow direction is in the $+z$-direction. Diagram is not to scale.

The side plates are not included in the coupled model. This is due, in part, to the issue of how to include them in the model. The contact interface with the fuel plate could easily be appended to the threedimensional fuel plate domain. The coolant domain, however, homogenizes the $s$-direction. There would be no physically meaningful way to attach them to the coolant domain. This simplifying decision is validated by the 3D model. Energy balances performed on the coolant-facing surfaces of the side plates show that the heat transfer into both to be minimal compared to both the overall entropy change in the coolant channel and the heat generation in the fuel meat. 
The use of unions and assemblies - continuous and discontinuous inter-component mesh boundariesis the same in the fuel plate as in the 3D model. The coolant domain's three components are joined via union resulting in a single continuous mesh throughout.

\section{B. Inputs}

The coupled model had the same inputs as the 3D model for initial conditions as well as inlet and exit conditions and nuclear heat generation. The only major difference is the addition of an involute coordinate system which was imported into the model for use in implementing the interdimensional coupling mechanism. Since there is no direct way to translate from an $x-y$ Cartesian coordinate system to an $s$ - $t$ involute coordinate system, the impromptu coordinate had to be generated via a contour plot. A table of points with structured $\theta$ and $t$ values was created in Matlab. The $x$ - and $y$-coordinates were then calculated for each point and stored in a matrix. The grid that this produces is shown in Fig. 6.2.

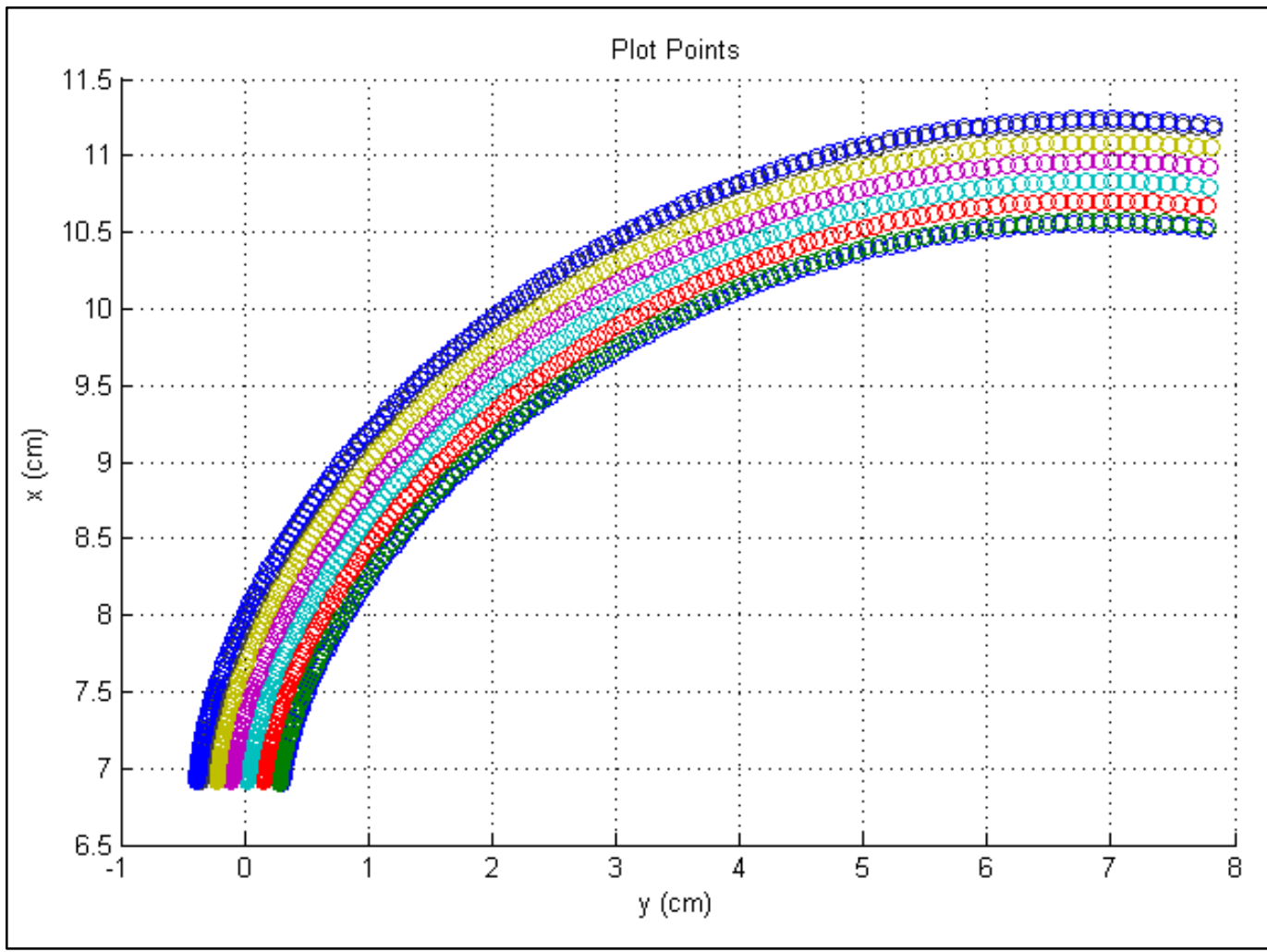

Fig. 6.2. Involute Coordinate Contour Grid Points. Each group of colored markers is associated with a constant $t$ value in which $t$ (in) $=-0.12,-0.11,-0.06,-0.01,0.04,0.09,0.14,0.15$. $\theta=0.00$ to 1.65 by 0.01 radian increments for each group. 
All four coordinate values $(x, y, \theta$, and $t)$ were then imported into COMSOL. The $x$ - and $y$-coordinates were treated as independent variables while $\theta$ and $t$ were treated as dependent functions. This allowed for a contour map of each involute coordinate to be created. These are shown in Figs. 6.3 and 6.4.

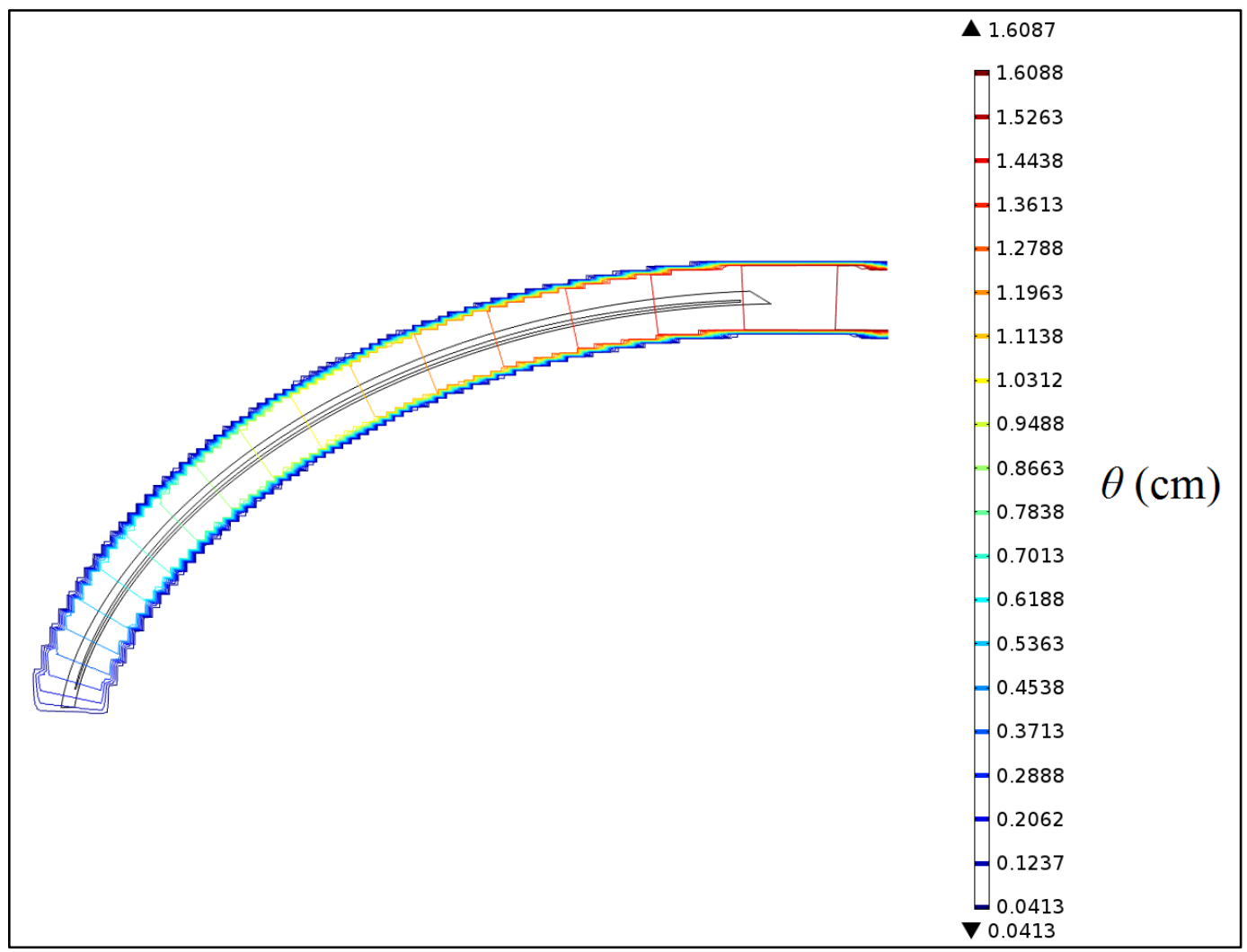

Fig. 6.3. 9-Coordinate Contour Map. The involute geometry is shown for reference.

Each coordinate map was made much larger than the involute geometry, which is shown underlain for reference. This was due primarily to the jaggedness of the contours on the edges of the maps where the coordinate grid ends. In order to prevent these discrepancies from infiltrating into the solution domain the boundaries were expanded to ensure smooth contours throughout. Once the functions $\theta(x, y)$ and $t(x, y)$ exist they can be referenced in COMSOL the same as a coordinate. The involute coordinate function $s(\theta, t)$ was created using Eqn. (3.17). 


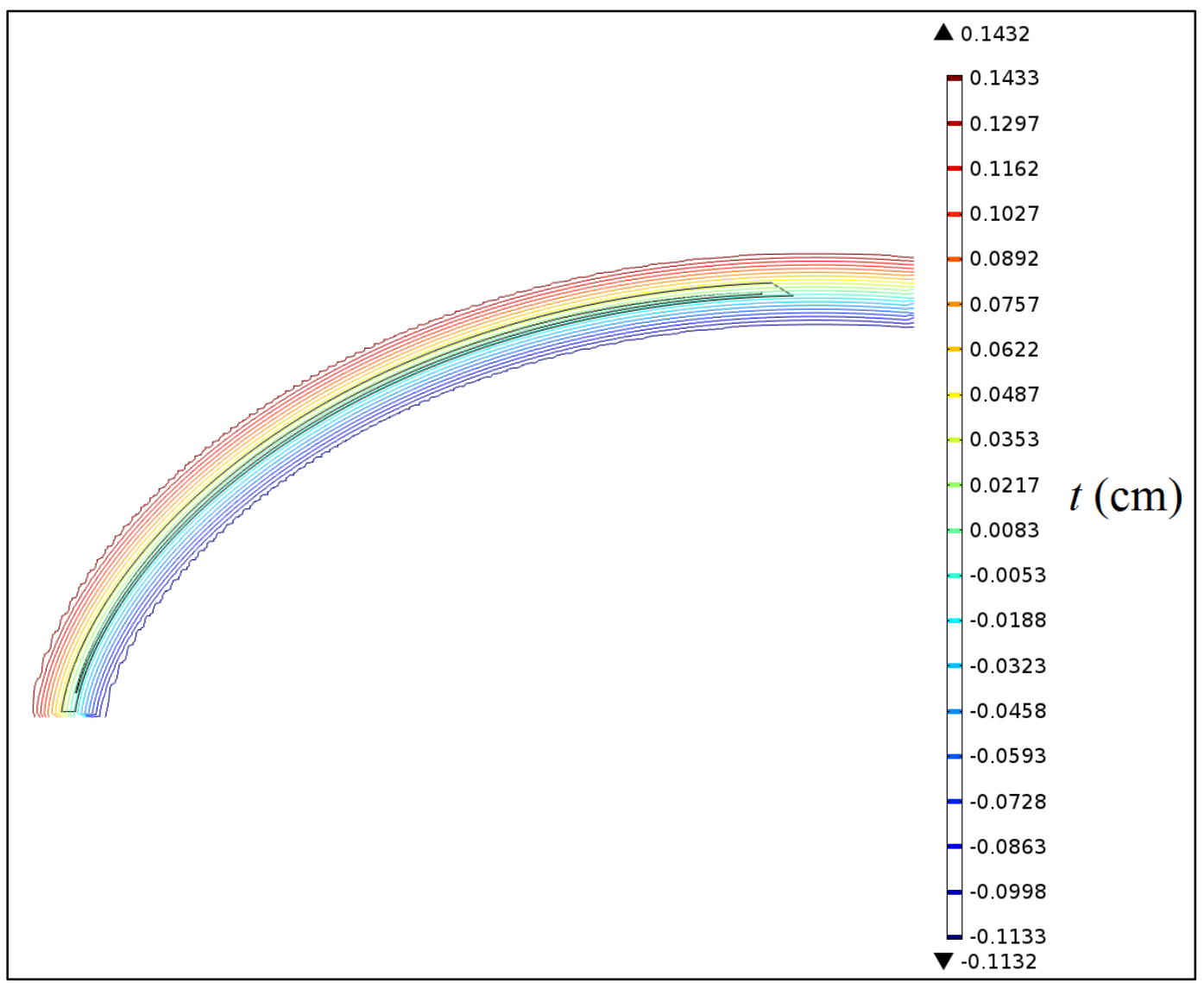

Fig. 6.4. $t$-Coordinate Contour Map. The involute geometry is shown for reference.

The other additional model input is the channel width as a function of axial position, $w_{c}(z)$. It is used in averaging flow properties across the width of the $2 \mathrm{D}$ channel slice. The channel width is constant for the length of the entire fuel plate except at the rounded leading edge (the right-hand side of coolant region 1 in Fig. 6.1). Therefore, the channel width function defines the axially-varying width along the length of the rounded leading edge. Constant-value extrapolations are used from the boundary values of the function to define the constant width along the rest of the length of the fuel plate.

The width of one-half of the rounded leading edge, $w_{r}$, is given as a function of its axial position:

$$
w_{r}(z)=\sqrt{r_{c}^{2}-\left(z-z_{o}\right)^{2}}
$$

where $r_{c}$ is the radius of the rounded leading edge and $z_{o}$ is the leading edge segment's axial center of rotation. The inverse of this width, the void width, $w_{v}$, is calculated by

$$
w_{v}(z)=r_{c}-w_{r}(z)
$$


Both functions are shown in Fig. 6.5.

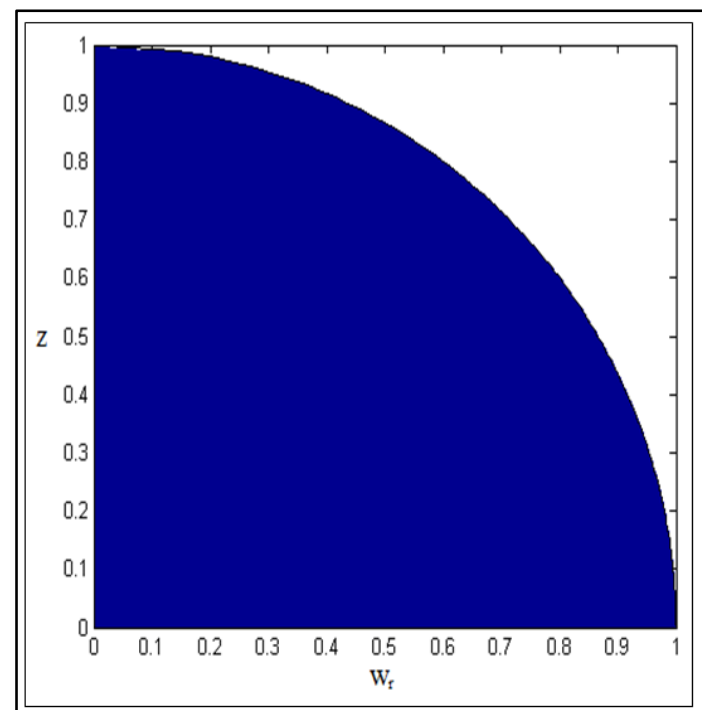

(A) Leading Edge Width

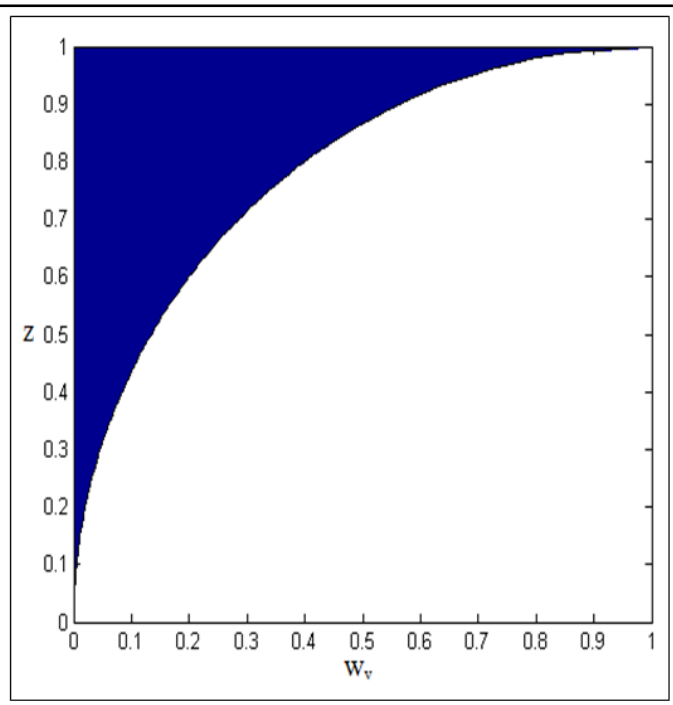

(B) Leading Edge Void Width

Fig. 6.5. Axially-Dependent Leading Edge Widths. $r_{c}$ is set to 1 and $z_{o}$ to 0 . Flow direction is in the $-z$-direction.

At its widest (at the stagnation point at the tip of the rounded leading edge) the void width is $4 r_{c}$. At its narrowest (once the rounded leading edge has reached the axially-constant width that it will maintain for the length of the fuel plate) this width is $2 r_{c}$. The channel width in $\mathrm{cm}$ is thus provided by

$$
w_{c}(z)=4 r_{c}-2 \sqrt{r_{c}^{2}-\left(z-z_{o}\right)^{2}},-5.08 \leq z \leq-5.0165
$$

where $z_{o}$ equals $-5.0165 \mathrm{~cm}$. The channel width is only applicable for axial positions bounded by the fuel plate. Even though the function returns values in the entrance and exit coolant regions, they are irrelevant. The final function is shown in Fig. 6.6. 


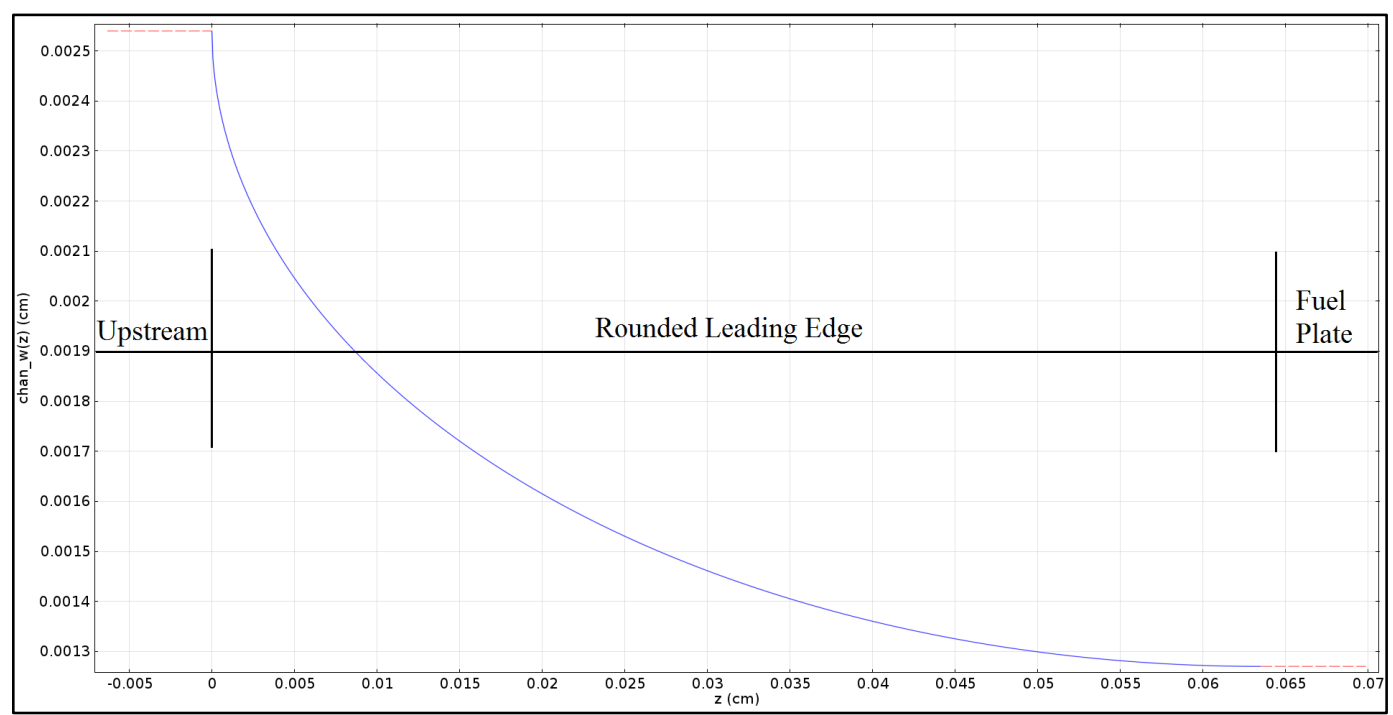

Fig. 6.6. Channel Width Input Function. Flow direction is in the $+z$-direction. The $z$ coordinate in the figure is a localized coordinate system, not the global $s-t-z$ system.

\section{Interdimensional Model Coupling}

The interdimensional model coupling mechanism functions in two directions: from the surface of the fuel plate to the surface of the coolant channel (3D-to-2D) and from bulk values in the coolant channel back to the surface of the fuel plate (2D-to-3D). In this way it produces a looped dependency between the two models in which their respective outputs and inputs are iteratively solved along with the domains to which they belong until the coupled system reaches a satisfactory equilibrium state.

Labeling the respective coupling mechanisms as such (3D-to-2D and 2D-to-3D) can be slightly misleading. The fuel plate-to-coolant channel coupling, for instance, involves the collection of variables on a $2 \mathrm{D}$ surface boundary ( $s-z$ plane) that are then reformatted and applied to a $1 \mathrm{D}$ edge boundary $(t-z$ plane). It would not be incorrect to think of this as a 2D-to-1D coupling based on the dimensions of the collected and applied coupling variables.

The reverse coupling mechanism, however, involves the collection of fluid properties that are inherently 2D that are then reformatted and applied to a 2D surface. The destination surface exists in the $s-z$ plane and the coolant channel in the $t-z$ plane, however. Coupling variables can only be passed in a dimension that is shared between the two domains. In this case the axial dimension $z$ is the only common dimension shared between the two. As with the first coupling mechanism, the result is a 2D-to-1D coupling. The respective coupling mechanisms are, therefore, most easily identified based on the dimensionality of the two respective domains, fuel plate (3D) and coolant channel (2D). 
The 3D-to-2D coupling will be considered first. In many ways it is the simpler of the two couplings. Primarily, only a single variable, versus four in the reverse direction, is being coupled. Additionally, the manner in which the reformatted variable is applied to its destination domain is the simpler of the two. The only communication from the fuel plate to the coolant channel is in the passing of surface temperature values, $T_{\text {surf }}$. Inherently, surface temperature is a function of $s$ and $z_{p}$, where a subscript $p$ denotes a coordinate in the fuel plate domain. The original surface temperature variable is given by

$$
T_{\text {surf }}=T\left(s, z_{p}\right)
$$

The two-dimensional axial surfaces in the fuel plate domain become one-dimensional axial edges in the coolant domain. The $T_{\text {surf }}$ variable must have its dependency in the $s$-direction removed in order to be applied to these edge boundaries. This can be achieved by integrating in the $s$-direction to produce a new variable, $\eta_{T}$, given by

$$
\eta_{T}\left(z_{p}\right)=\int_{s_{1}}^{s_{2}} T_{\text {suff }}\left(s, z_{p}\right) d s .
$$

where $s_{1}$ is the $s$-coordinate at the fuel plate-inner side plate boundary and $s_{2}$ is the $s$-coordinate at the fuel plate-outer side play boundary. This new variable has the correct spatial dependency but not the correct units $-\mathrm{K}-\mathrm{m}$ versus the desired $\mathrm{K}$. To rectify this, it is divided by the length it was integrated over. This produces a variable of spanwise-averaged surface temperature values as a function of axial position:

$$
\bar{T}_{\text {surf }}\left(z_{p}\right)=\frac{\eta_{T}\left(z_{p}\right)}{\Delta s}
$$

where $\Delta s$ is the span of the fuel plate. This spanwise-averaged temperature variable is then applied directly to the coolant domain boundaries such that

$$
T_{\text {surf }}\left(z_{c}\right)=\bar{T}_{\text {surf }}\left(z_{p}\right)
$$

where a subscript $c$ denotes a coordinate in the coolant channel domain. The entire process is shown in the flow chart in Fig. 6.7. 


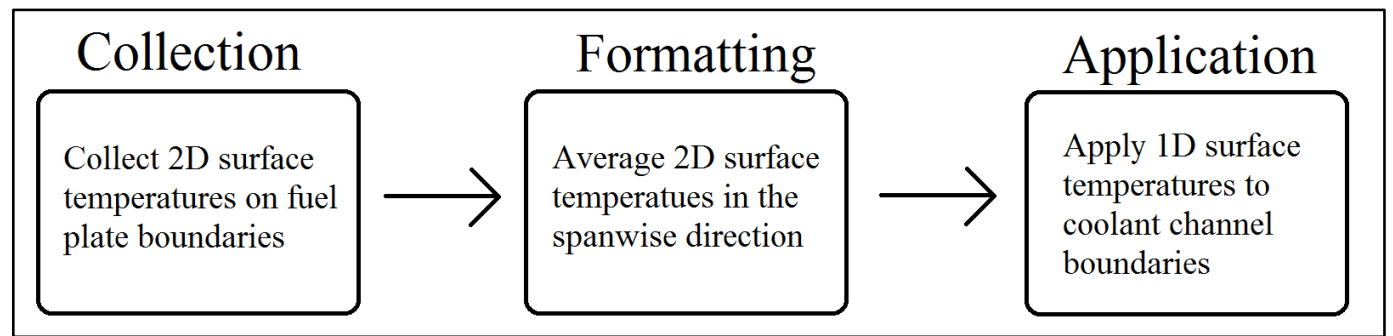

Fig. 6.7. 3D-to-2D Coupling Mechanism Flow Chart.

This procedure is applicable for every axial surface on the fuel plate, including the rounded leading edge. Returning to Fig. 6.1 shows, however, that the trailing edge of the fuel plate (in the fuel plate domain) is confined to the $s$ - $t$ plane. Surface temperatures are thus given as a function of $s$ and $t$ instead of $s$ and $z$

$$
T_{\text {surf }}=T\left(s, t_{p}\right)
$$

The $t$-coordinate is now the common coordinate shared between the two domains. The Surface temperature values are once again averaged along the span of the plate and stored as a function of $t$. The final application then takes on the form

$$
T_{\text {surf }}\left(t_{c}\right)=\bar{T}_{\text {surf }}\left(t_{p}\right)
$$

Additionally, the coupling of the convex and the concave sides must be kept separate so that they are allowed to vary independently of one another. Therefore there are technically two 3D-to-2D coupling variables, one for each side of the fuel plate. Surface temperature values are applied to the coolant channel edge boundaries via Dirichlet constraints. The coupling process, however, also inherently conserves the heat flux

The reverse coupling mechanism is the $2 \mathrm{D}$-to-3D coupling in which coupling variables are passed from the coolant to the fuel plate in order to complete the feedback loop. By the merit of its construction as a two-dimensional $t$ - $z$ slice, the coolant channel is, in essence, already representative of bulk flow properties in the $s$-direction. Given the coolant channel $(t-z)$ and the fuel plate surfaces $(s-z)$, the only common variable is again the axial coordinate $z$. In order to remove the $t$-coordinate dependency from the coolant channel flow variables, bulk values are taken in the $t$-direction as a function of $z$. The four coupling variables are $T, \rho, \mu$, and $\operatorname{Pr}$.

The definition for bulk temperature in a flow is 


$$
T_{b}=\frac{\dot{I}}{\dot{C}}=\frac{\text { total enthalpy flow rate }}{\text { heat capacity flow rate }}
$$

In the $t-z$ coolant channel, for variable-property flow, this takes on the form ${ }^{68}$

$$
T_{b}\left(z_{c}\right)=\frac{\int_{-w_{c}\left(z_{c}\right) / 2}^{+w_{c}\left(z_{c}\right) / 2} \rho u C_{p} T d t}{\int_{-w_{c}\left(z_{c}\right) / 2} \rho u C_{p} d t} .
$$

This operation, however, is extremely expensive in that each integral requires its own coupling mechanism. Every additional coupling mechanism in the model increases the solution time by a nontrivial fraction. This is especially true of the two mechanisms contained in this formulation given the relatively large number of operations contained within (compared to the coupling mechanism in Eqn. (6.5) in which only a single variable is collected). It is more computationally efficient to calculate bulk temperature in the same manner as surface temperature. While less accurate, the difference in passed bulk temperature values is small enough that the use of the more rigorously physical formulation is not warranted.

In this simplified formulation, bulk temperature, or any coupling flow variable, $\phi$, represented by

$$
\phi=\phi\left(t_{c}, z\right)
$$

can be passed according to

$$
\begin{gathered}
\eta_{\phi}\left(z_{c}\right)=\int_{-w_{c}\left(z_{c}\right) / 2}^{+w_{c}\left(z_{c}\right) / 2} \phi\left(t_{c}, z\right) d t \\
\phi_{b}\left(z_{c}\right)=\frac{\eta_{\phi}\left(z_{c}\right)}{w_{c}\left(z_{c}\right)},
\end{gathered}
$$

and

$$
\phi_{b}\left(z_{p}\right)=\phi_{b}\left(z_{c}\right)
$$

The 2D-to-3D coupling process is shown in Fig. 6.8 . 


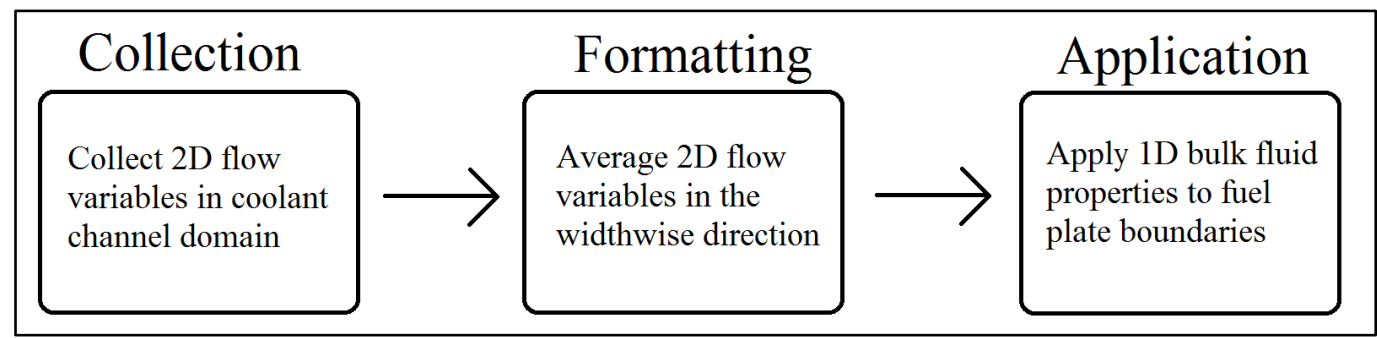

Fig. 6.8. 2D-to-3D Coupling Mechanism Flow Chart.

Unlike the 3D-to-2D coupling, the process of collecting and passing variables from the trailing edge in the coolant domain to the fuel plate domain is unchanged. Flow properties across both surfaces are still averaged in the $t$-direction and stored as a function of $z$. Therefore, a single value for each flow property is applied to the entire trailing edge.

The coupled flow properties are applied to the fuel plate surface via a simple convective boundary condition

$$
q_{n}^{\prime \prime}\left(z_{p}\right)=h\left(T_{\text {surf }}-T_{b}\right)
$$

where the heat transfer coefficient, $h$, is given as

$$
h\left(z_{p}\right)=\mathrm{Nu} \frac{\lambda}{D_{h}}
$$

where $D_{h}$ is the hydraulic diameter and the Nusselt number, $\mathrm{Nu}$, comes from a modified form of the Hausen correlation. ${ }^{73}$ The original Hausen correlation is given as

$$
\mathrm{Nu}_{b m}=0.116 \operatorname{Pr}_{b}^{1 / 3}\left(\frac{\mu_{b}}{\mu_{w}}\right)^{0.14}\left(\operatorname{Re}_{b}^{2 / 3}-125\right)\left[1+\left(\frac{D_{h}}{L_{h}}\right)^{2 / 3}\right]
$$

where $L_{h}$ is heated length, a subscript $m$ denotes a mean axial value, and a subscript $w$ denotes a wall value. The original Hausen correlation is valid in both the turbulent and transition flow regimes. The modified Hausen correlation was derived by Gambill and Bundy $^{74}$ as a result of experimental work performed in the study of turbulent flow in thin rectangular channels. The range of conditions over which Gambill and Bundy experimentally verified the applicability of the modified Hausen correlation is shown in Table 6.1. The range of nominal HFIR operating conditions are also shown for comparison. 


\begin{tabular}{|c|c|c|}
\hline \multicolumn{2}{|c|}{ Table 6.1: Modified Hausen Correlation Validation Range } \\
\hline & $\begin{array}{c}\text { Experimental } \\
\text { Conditions }\end{array}$ & $\begin{array}{c}\text { Nominal HFIR } \\
\text { Conditions }\end{array}$ \\
\hline$q^{\prime \prime}\left(\mathrm{W} / \mathrm{cm}^{2}\right)$ & $32-2334$ & $50-500$ \\
\hline$D_{h}(\mathrm{~cm})$ & $0.191-0.267$ & 0.249 \\
\hline$u(\mathrm{~m} / \mathrm{s})$ & $3.1-26.0$ & $7.8-18.0$ \\
\hline $\mathrm{Re}_{z}$ & $9 \times 10^{3}-2.7 \times 10^{5}$ & $6.9 \times 10^{4}-1.0 \times 10^{5}$ \\
\hline$p(\mathrm{MPa})$ & $0.11-3.91$ & $2.51-3.17$ \\
\hline$L_{h}(\mathrm{~cm})$ & $30.5-45.7$ & 50.8 \\
\hline
\end{tabular}

The only criteria in which nominal HFIR operating conditions fall outside of the experimentally verified range is in the heated length. But even then the disparity is only $10 \%$ of the maximum verified heated length.

The modified Hausen correlation provides a local heat transfer coefficient where the original Hausen provides a mean value. It can be derived by first assuming that the mean value is the average of the local values, $N_{b z}$, for the entire heated length of the plate. Thus

$$
\mathrm{Nu}_{b m}=\frac{\int_{0}^{z / D_{h}} \mathrm{Nu}_{b z} d\left(\frac{z}{D_{h}}\right)}{\frac{z}{D_{h}}} .
$$

The modified local Hausen correlation can then be derived according to

$$
\mathrm{Nu}_{b z}=\frac{d\left[\mathrm{Nu}_{b m}\left(\frac{z}{D_{h}}\right)\right]}{d\left(\frac{z}{D_{h}}\right)} .
$$

If the following substitution is made in Eqn. (6.18)

$$
\Pi=0.116 \operatorname{Pr}_{b}^{1 / 3}\left(\frac{\mu_{b}}{\mu_{w}}\right)^{0.14}\left(\operatorname{Re}_{b}^{2 / 3}-125\right)
$$

then the original form of the Hausen correlation can be given as

$$
\mathrm{Nu}_{b m}=\Pi\left[1+\left(\frac{D_{h}}{z}\right)^{2 / 3}\right]
$$


such that the derivative with respect to $z / D_{h}$ becomes

$$
\mathrm{Nu}_{b z}=\frac{d\left\{\Pi\left[1+\left(\frac{D_{h}}{z}\right)^{2 / 3}\right]\left(\frac{z}{D_{h}}\right)\right\}}{d\left(\frac{z}{D_{h}}\right)} .
$$

Simplifying yields

$$
\mathrm{Nu}_{b z}=\frac{d\left\{\Pi\left[\frac{z}{D_{h}}+\left(\frac{z}{D_{h}}\right)^{1 / 3}\right]\right\}}{d\left(\frac{z}{D_{h}}\right)}
$$

and differentiating with respect to $z / D_{h}$ yields

$$
\mathrm{Nu}_{b z}=\Pi\left[1+\frac{1}{3}\left(\frac{D_{h}}{z}\right)^{2 / 3}\right]
$$

The final form of the modified Hausen correlation is

$$
\mathrm{Nu}_{b z}=0.116 \operatorname{Pr}_{b z}^{1 / 3}\left(\frac{\mu_{b z}}{\mu_{w z}}\right)^{0.14}\left(\operatorname{Re}_{b z}^{2 / 3}-125\right)\left[1+\frac{1}{3}\left(\frac{D_{h}}{z}\right)^{2 / 3}\right]
$$

where $D_{h}$ is the hydraulic diameter of the full 3D coolant channel, not the channel width of the 2D coolant slice. The modified form is very similar to the original form. The only two alterations are the addition of a $1 / 3$ coefficient on the last term and a subscript $z$ on every flow variable to denote that it is now a local value rather than a mean axial value. Values for $\operatorname{Pr}_{b z}$ and $\mu_{b z}$ are provided directly from the coupled variables for both. Values for $\operatorname{Re}_{b z}$ are calculated using coupled values for $\rho_{b z}$ and $\mu_{b z}$ according to

$$
\operatorname{Re}_{b z}=\frac{\rho_{b z} u_{b m} D_{h}}{\mu_{b z}}
$$

where $u_{b m}$ is bulk, mean axial velocity. The mean axial velocity is used, in lieu of an axially-dependent velocity, because the convective boundary condition requires a constant value for the external flow magnitude. Attempts to input velocity as a function of $z$ failed after a single iteration. While an axiallydependent bulk flow velocity would be able to account for developing flow in the hydrodynamic entrance region, its exclusion is required by the code itself. As would be expected for such a high aspect ratio 
channel, the hydrodynamic entrance region turns out to be small, as will be shown in Section VII. Given this necessary simplification, $u_{b m}$ was set to twice the inlet velocity. The wall viscosity, $\mu_{w z}$, is calculated based on wall-adjacent flow conditions.

A key component in the successful implementation of the interdimensional model coupling mechanism is the exclusion of all of the coupling variables from the Jacobian solution matrix. Doing so minimizes the additional computational cost associated with the creation of the coupling variables. Failure to do so increases the computation time by more than a factor of 2 . Without the exclusion of coupling variables from the Jacobian a mesh-converged solution would not be possible. The exclusion of any model variable can be forced through the use of a nojac operator in its definition. As would be expected, the loss of such information in the Jacobian can lead to solution instabilities, sometimes severe. This is not an issue with the coupled model given the nature of the coupling variables in which they serve only as adaptive boundary conditions with which to drive internal domain solutions.

The implementation of both coupling mechanisms creates a looped dependency between the models which, like the solutions of the internal domains, can converge to an equilibrium value through an iterative process. The complete coupling loop is shown in Fig. 6.9.

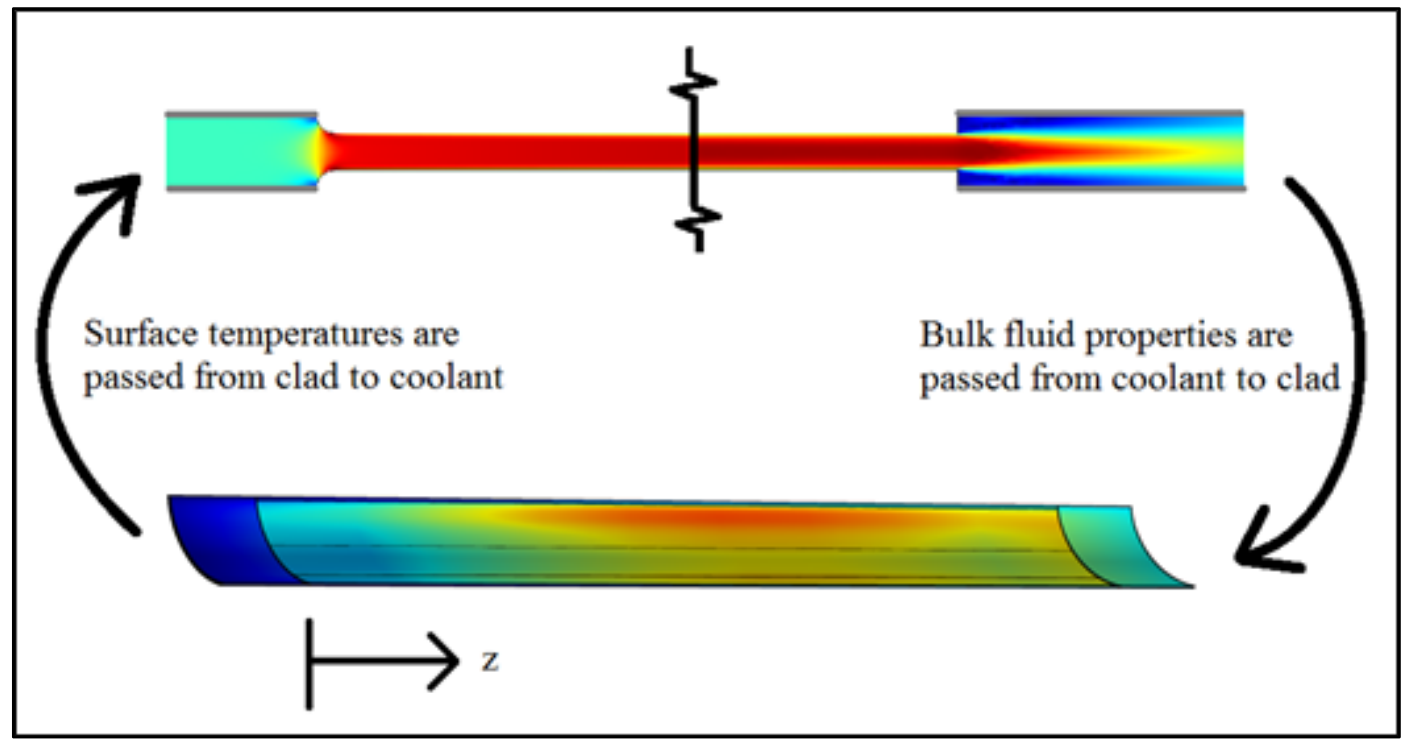

Fig. 6.9. Model Coupling Interdependency Loop. Flow is in the $+z$-direction.

The full iterative solution process implemented in the coupled model is shown in Fig. 6.10. 


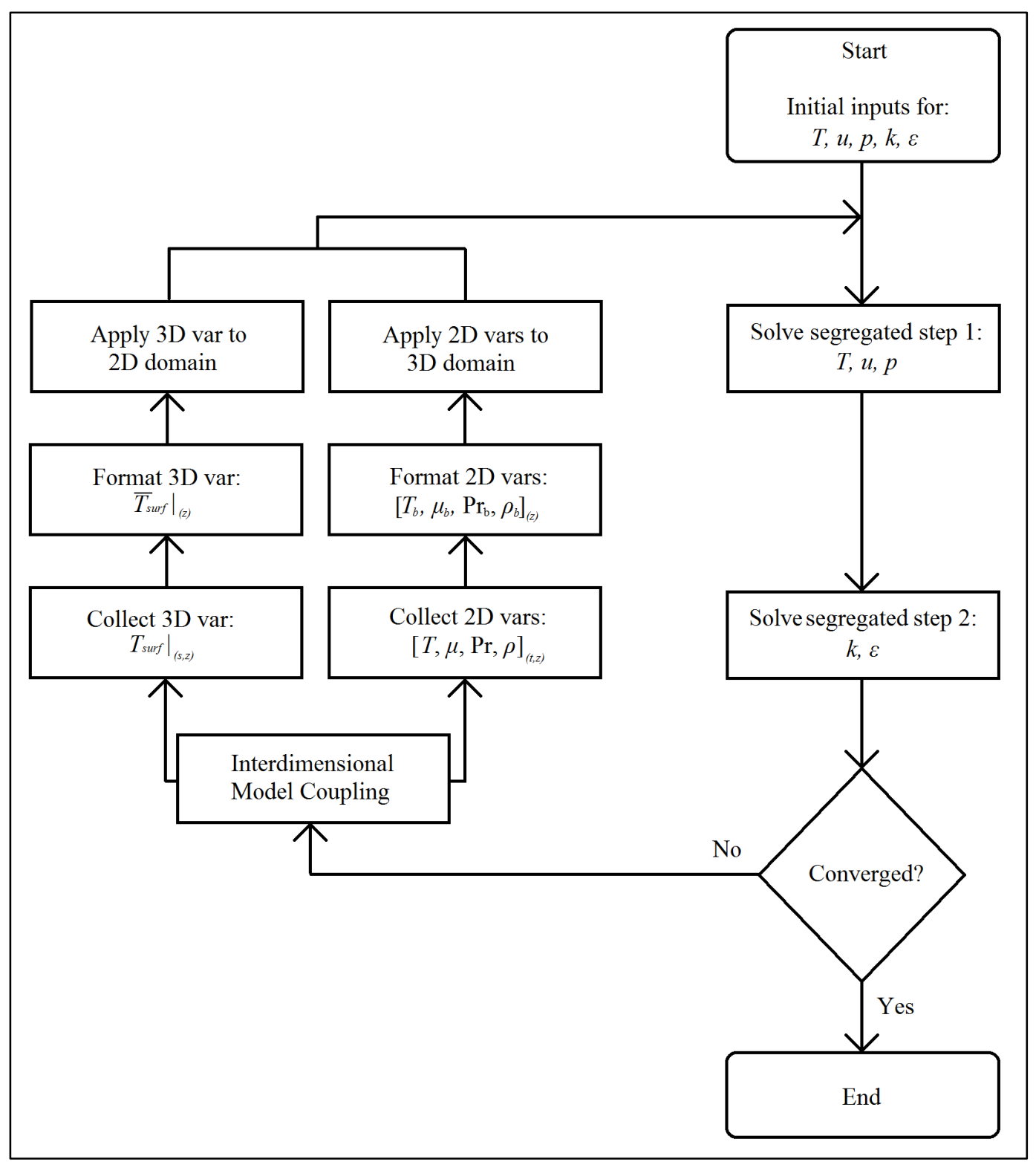

Fig. 6.10. Coupled Model Solution Process Flow Chart.

\section{Physics}

The same physics govern the coupled model that govern the 3D model. The only difference is in the application of boundary conditions. The coolant has the same inlet and outlet conditions. The axial boundary edges above and below the fuel plate coincide with the plate midpoint and are governed by periodic mass and heat flow conditions. The fuel plate boundary edges are modeled as no-slip, impermeable walls with imposed coupled heat flow conditions. 
In the fuel plate, every coolant-adjacent surface is specified using the previously detailed convective boundary condition. The two surfaces that would mate with the side plates in the 3D model are modeled as thermally insulated boundaries. This turns out to have little effect on the model. Given the extremely high-aspect ratio in the plate, heat conduction tends to occur predominantly in the $t$-direction, or perpendicular to the clad surface. Heat conduction, as will be shown in Section VII, is minimal in the $s$ direction, or along the length of the clad. As such, stopping off the heat outlet that traditionally exists via the fuel plate-side plate shared surface has a negligible effect on the model.

\section{E. Meshing}

An important feature of the coupled model is the separation of its two solution domains, solid and liquid. This separation allows for separate basis functions to be used in each domain. Namely, the solid domain, in which the heat generation-resultant quadratic temperature profile is found, is able to use quadratic functions while the coolant can still use linear functions. The solid domain mesh has the same structure as in the 3D model. Meshes are mapped to $s$ - $t$ faces, where applicable, and extruded using the 2X MCNP axial mesh distribution. The maps in the fuel meat and the unfueled plate are structured meshes while the plate surrounding the meat is mapped using a free-triangular mesh. The rounded leading edge is meshed using free-tetrahedral elements. The mesh resolution in all of these domains is lower than in the 3D model, however, given the ability of the quadratic basis functions to provide a more accurate approximation using fewer elements.

As previously mentioned, the Coolant domain is split into three sections: upstream, fuel plate, and downstream. The upstream and downstream sections are mapped using unstructured triangular meshes. The fuel plate section was originally meshed using the same triangular elements. This proved extremely inefficient, however, given the high-aspect ratio of the channel. A second mesh was created consisting of a structured rectangular mesh. The mesh was extruded axially using both constant-width cells and the $2 \mathrm{X}$ MCNP distribution. A boundary layer was added to all three fuel plate section meshes along the wall boundaries. The two mesh types, free-triangular and structured, are shown in Fig. 6.11. 


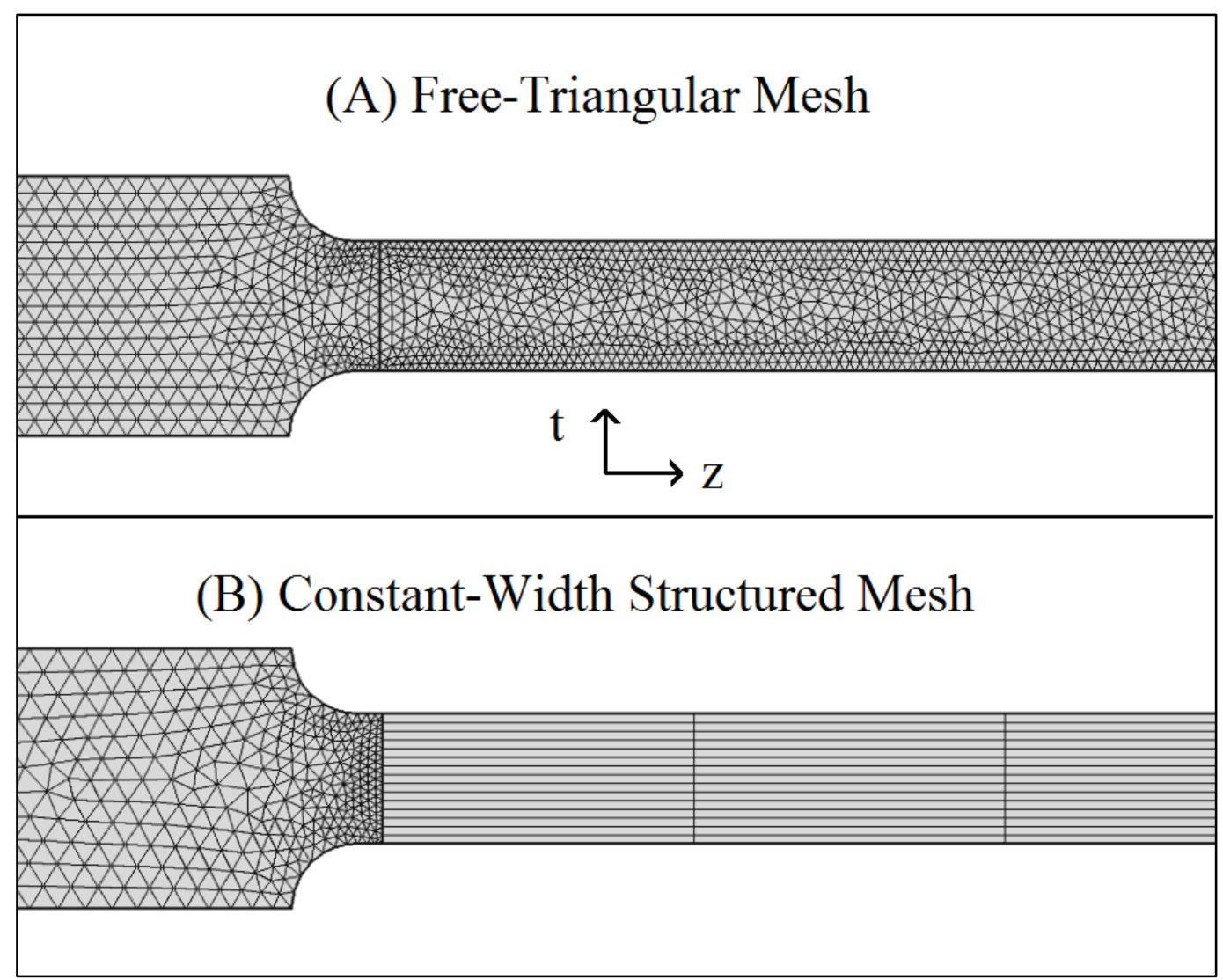

Fig. 6.11. Coupled Model Coolant Mesh Comparison.

An interesting limitation of the coupling mechanism is that it is compatible with triangular mesh elements. This means it cannot be used on a surface that contains rectangular mesh elements. In the coolant, this requires the insertion of diagonal mesh walls to divide a single rectangular element into two triangular elements. In the solid domain, 3D volume mesh elements must be cut in half. A detailed view of a converted boundary-layer mesh is shown in Fig. 6.12. 


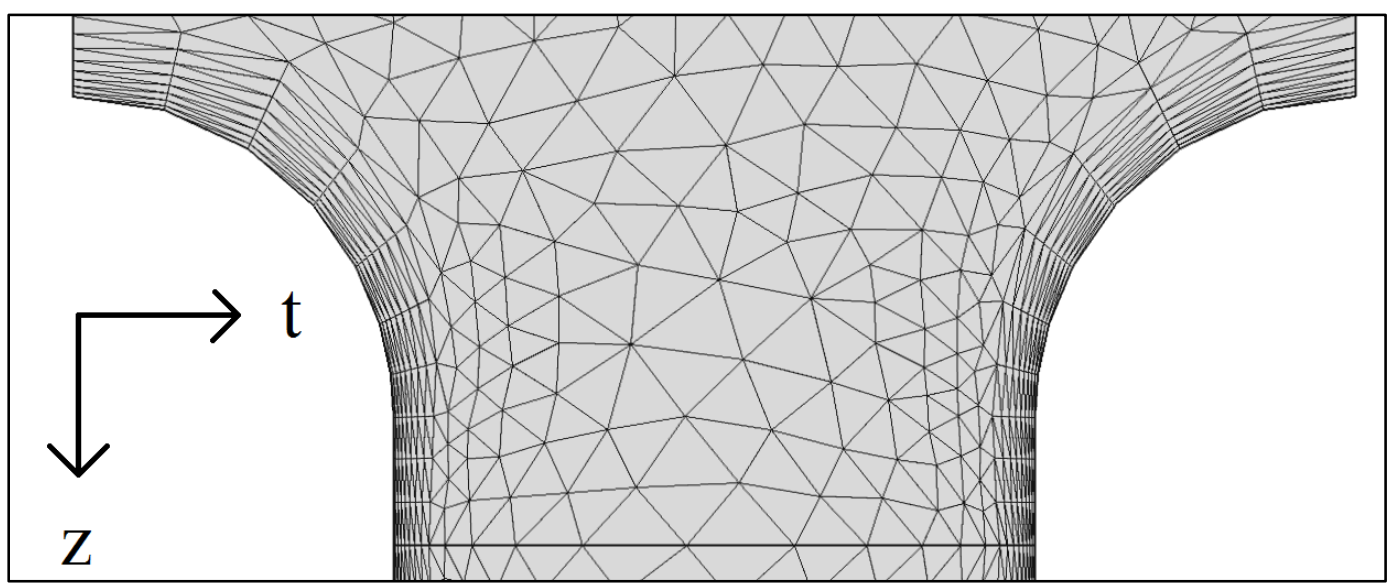

Fig. 6.12. Coupled Model Converted Boundary Layer Detail.

\section{F. Paneling}

The biggest drawback of the coupled model is the homogenizing effect the coolant channel slice has on the fuel plate temperature profile. Surface temperature values passed from the clad to the coolant must necessarily drop a dimension given the dimensional disparity between the two domains. The passed variable cannot have a dimensionality greater than either its source or destination. This is also true in reverse. The problem, however, is that in this latter case the passed variable has a lower dimensionality than the boundary it is being passed to. In essence, the boundary is capable of storing more information than the passed variable is able to provide it. The result is that an entire dimension's worth of information is wasted.

This means that the incoming bulk properties, especially bulk temperature, have a profile proportional to spanwise-averaged surface temperature rather than local surface temperature. Thus, at any point where the local surface temperature is greater than the spanwise-averaged surface temperature, the incoming bulk temperature is lower than it should be. Conversely, at any point where the local surface temperature is less than the spanwise average, the incoming bulk temperature is higher than it should be. This has a smoothing effect on the surface temperature profile, blunting its maxima and minima. This smoothing effect propagates into the interior of the fuel plate and fuel meat as well. Given the model's primary intent of predicting maximum temperatures, this is an undesirable consequence of the model's construction.

An attempt was made to reintroduce a certain level of spanwise fidelity that the coupled model's construction had erased. In this model, the paneled model, the surface of the fuel plate is split into a finite number of axial strips as shown in Fig. 6.13. 


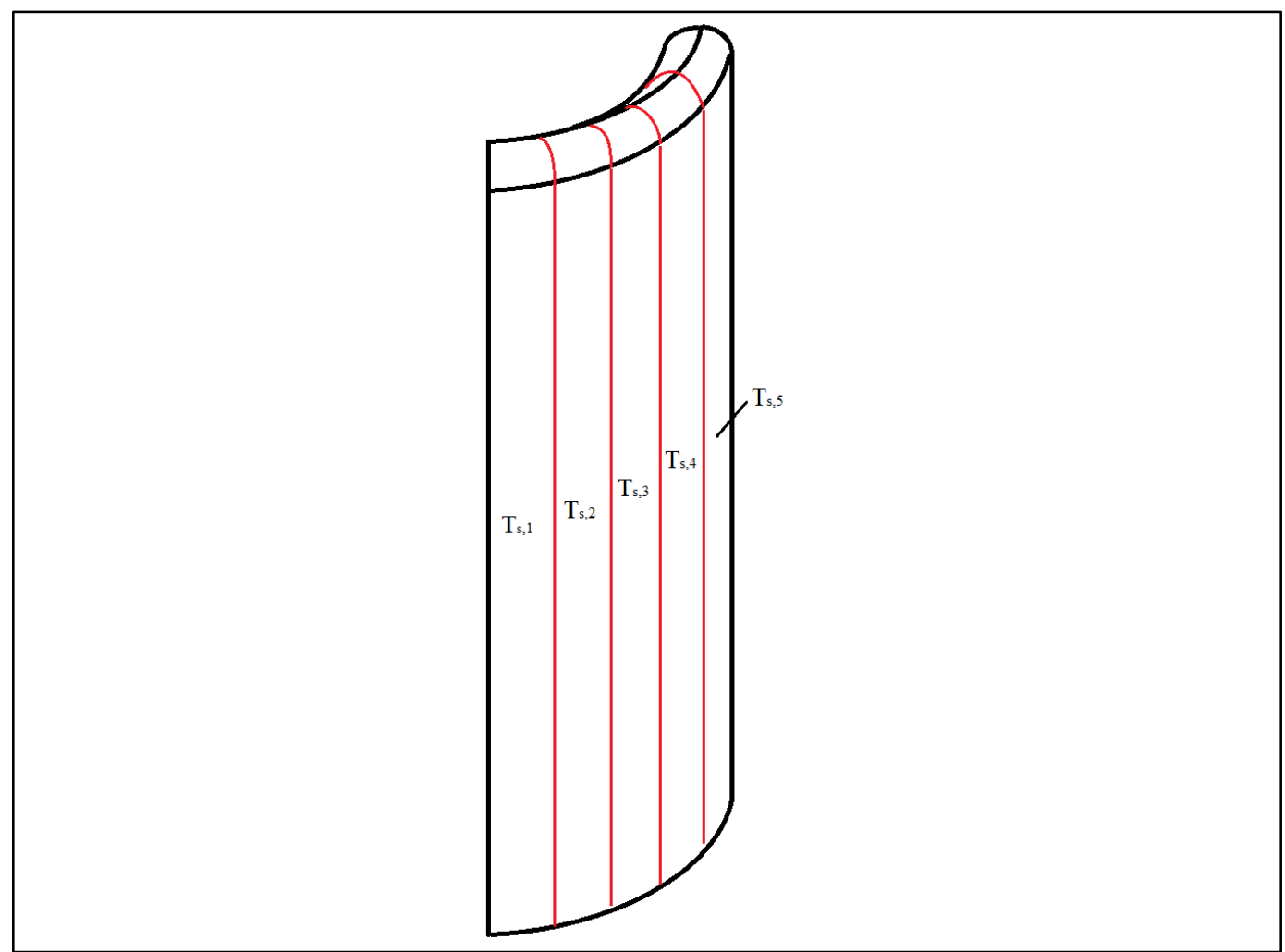

Fig. 6.13. Fuel Plate Paneling. Depicted is a five-panel decomposition. Figure is not to scale.

Each plate surface strip, along with its counterpart on the opposite side of the fuel plate, is paired with its own coolant channel slice. Plate surface temperatures are averaged across each strip and passed to their respective slice. Bulk flow properties are then passed back from slice to strip. Instead of relying on a single spanwise bulk flow, the model is split into a finite number of bulk flows. If the number of panels were increased to infinity one would essentially recreate the original 3D channel. By creating a small number of slices, however, a measure of that spanwise variation in flow and plate surface properties is allowed to manifest itself without the burden of solving the entire flow channel.

The dependency loop for a two-panel model is shown in Fig. 6.14. Ultimately, the design proved infeasible due to two factors. Firstly, increasing the number of panels beyond two increases the model's computational cost to a degree that it is no longer a viable improvement over the $3 \mathrm{D}$ model. This is due to the fact that every additional panel precipitates the need for an entire new coolant channel slice, thus doubling the degrees of freedom from that half of the model. While the coolant channel slice is the simpler of the two domains, from a geometry standpoint, the fact that it must solve the N-S equations means that the additional degrees of freedom are significant. Secondly, as will be shown in greater detail 
in Section VII, even a two-panel configuration does not produce the desired results due to the high-aspect ratio of the geometry.

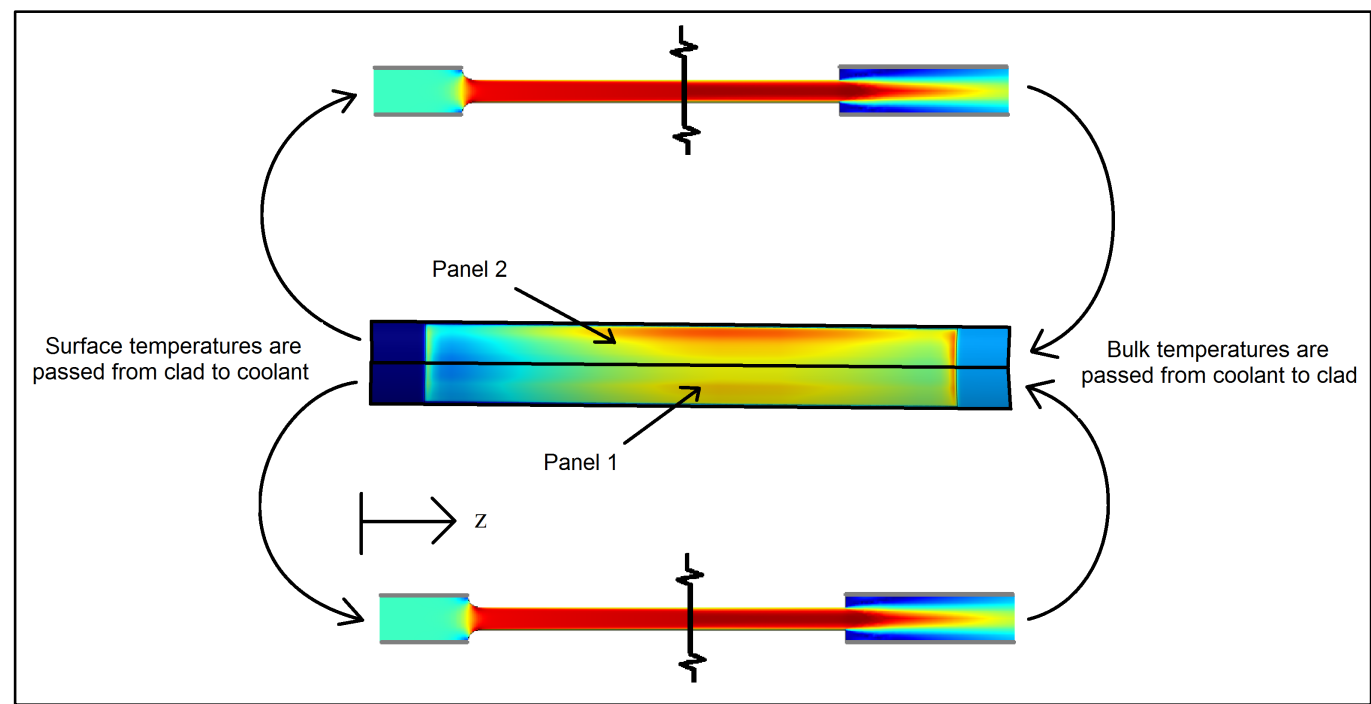

Fig. 6.14. Fuel Plate Paneling Diagram. 


\section{Results}

\section{A. Error Evaluation}

Before model results are presented, the means and methods of evaluating the validity of a given model configuration must be specified. This is achieved primarily through the assessment of a model's energy balance. For steady-state models such as those under consideration here this means that the conservation of energy takes on the form

$$
\dot{E}_{\text {in }}+\dot{E}_{\text {gen }}-\dot{E}_{\text {out }}=0
$$

where $\dot{E}$ is actually energy-per-unit-time and has units of power (W) and all terms are assumed to be positive. In order to break the model down further so as to trace energy imbalances to a specific domainfuel plate or coolant - this simple energy balance can be evaluated individually for each domain. The fuel plate has no incoming energy term but does have an energy generation term due to the nuclear heat production in the fuel meat. This generation term, $\dot{E}_{p, g e n}$, is given by

$$
\dot{E}_{p, g e n}=\iiint_{V} Q d V
$$

The outgoing energy for the fuel plate, $\dot{E}_{p, o u t}$, is the total heat transfer through the surface of the fuel plate given by

$$
\dot{E}_{p, \text { out }}=\iint_{A_{p}}\left(\boldsymbol{q}^{\prime \prime} \cdot \hat{\boldsymbol{n}}_{p}\right) d A
$$

where $\hat{\boldsymbol{n}}_{p}$ is the local outward surface normal vector for the fuel plate geometry and $A_{p}$ is the surface area of the fuel plate. This $\dot{E}_{\text {out }}$ term for the fuel plate becomes part of the $\dot{E}_{\text {in }}$ term for the coolant (the side plates are considered part of the coolant domain for energy balance purposes in the 3D model). The other $\dot{E}_{\text {in }}$ components come from the inlet enthalpy in the coolant and inlet heat transfer in the side plates. The total term is given in the $3 \mathrm{D}$ model by

$$
\left.\dot{E}_{c, \text { in }}\right|_{3 D}=-\left[\iint_{A_{p}}\left(\boldsymbol{q}^{\prime \prime} \cdot \hat{\boldsymbol{n}}_{c}\right) d A+\iint_{A_{\text {inlet }}}\left(\boldsymbol{u} \cdot \hat{\boldsymbol{n}}_{\text {inlet }}\right) \rho c_{p} T d A+\iint_{A_{s p, \text { inlet }}}\left(\boldsymbol{q}^{\prime \prime} \cdot \hat{\boldsymbol{n}}_{s p, \text { inlet }}\right) d A\right]
$$


where $\hat{\boldsymbol{n}}_{c}$ is the outward surface normal vector of the coolant channel geometry in contact with the fuel plate and is the opposite of $\hat{\boldsymbol{n}}_{p}$. The coolant domain does not possess an $\dot{E}_{\text {gen }}$ term. $\dot{E}_{\text {out }}$ in the $3 \mathrm{D}$ model's coolant is given by

$$
\left.\dot{E}_{c, \text { out }}\right|_{3 D}=\iint_{A_{\text {oulet }}}\left(\boldsymbol{u} \cdot \hat{\boldsymbol{n}}_{\text {outlet }}\right) \rho c_{p} T d A+\iint_{A_{\text {open }}}\left(\boldsymbol{u} \cdot \hat{\boldsymbol{n}}_{\text {open }}\right) \rho c_{p} T d A+\iint_{A_{s p, o u l e t}}\left(\boldsymbol{q}^{\prime \prime} \cdot \hat{\boldsymbol{n}}_{s p, \text { outlet }}\right) d A
$$

where the middle term is the outlet enthalpy along the open axial boundary beneath the inner side plate. Another way of looking at the energy balance in the coolant domain is to consider energy added to the system via the fuel plate and the change in enthalpy in the coolant and side plates between inlet and outlet. Ideally

$$
\dot{E}_{c, i n(\text { plate })}-\Delta \dot{H}_{c}=0
$$

where $\Delta \dot{H}_{c}$ is the change in enthalpy-per-unit-time in the coolant domain and the terms are given by

$$
\left.\dot{E}_{c, \text { in }(\text { plate })}\right|_{3 D}=-\iint_{A_{p}}\left(\boldsymbol{q}^{\prime \prime} \cdot \hat{\boldsymbol{n}}_{c}\right) d A=\dot{E}_{p, \text { out }}
$$

and

$$
\left.\Delta \dot{H}_{c}\right|_{3 D}=\left(\dot{E}_{c, \text { outlet }}-\dot{E}_{c, \text { inlet }}\right)+\left(\dot{E}_{s p, \text { outlet }}-\dot{E}_{s p, \text { inlet }}\right)+\dot{E}_{c, \text { open }} .
$$

Coolant energy values are formulated differently for the coupled model. Since the coolant is represented as a two-dimensional $t-z$ slice, boundary integrals become line integrals. These quantities must be multiplied by the span of the fuel plate axial surface, $\Delta s$, in order to maintain dimensional parity. The energy-in and enthalpy change terms become

$$
\left.\dot{E}_{c, \text { in }(\text { plate })}\right|_{\text {coupled }}=-\Delta s \int_{z_{1}}^{z_{2}}\left[\left(\mathrm{q}^{\prime \prime} \cdot \hat{\mathrm{n}}_{\text {concave }}\right)+\left(\mathrm{q}^{\prime \prime} \cdot \hat{\mathrm{n}}_{\text {convex }}\right)\right] d z
$$

and

$$
\left.\Delta \dot{H}_{c}\right|_{\text {coupled }}=\Delta s \int_{-w_{c}\left(z_{\text {inlet }}\right) / 2}^{+w_{c}\left(z_{\text {inle }} t\right) / 2}\left[\left(\mathrm{u} \cdot \hat{\mathrm{n}}_{\text {outlet }}\right) \rho c_{p} T-\left(\mathrm{u} \cdot \hat{\mathrm{n}}_{\text {inlet }}\right) \rho c_{p} T\right] d t=\dot{E}_{c, \text { outlet }}-\dot{E}_{c, \text { inlet }}
$$

where terms for the side plates and the open boundary beneath the inner side plate are neglected because such features are not present in the coupled model and $z_{1}$ and $z_{2}$ correspond to the axial coordinates for the 
top and bottom of the coupled boundary, respectively. The normal $\hat{\boldsymbol{n}}_{\text {concave }}$ is the outward facing normal vector for the coolant domain edge boundary corresponding to the concave surface of the fuel plate. The normal $\hat{\boldsymbol{n}}_{\text {convex }}$ corresponds to the opposite fuel plate edge boundary in the coolant domain. Whereas the energy leaving the fuel plate, $\dot{E}_{p, o u t}$, and the energy entering the coolant domain from the fuel plate, $\dot{E}_{c, \text { in (plate) }}$, are equal in the 3D model, the same assumption cannot be made in the coupled model. This is due to the fact that they are separated via the interdimensional model coupling mechanism in the coupled model.

Individual energy balances can be constructed for each domain such that

$$
\% e_{p}=\frac{\left|\dot{E}_{p, \text { out }}-\dot{E}_{p, \text { gen }}\right|}{\dot{E}_{p, \text { gen }}} \times 100 \%
$$

and

$$
\% e_{c}=\frac{\left|\Delta \dot{H}_{c}-\dot{E}_{p, o u t}\right|}{\dot{E}_{p, \text { out }}} \times 100 \%
$$

In both local energy balances, the incoming energy is assumed to represent the true energy. Any nonzero result from the energy balance is thus attributed to the outgoing term (loosely defined in the coolant domain). In the fuel plate, the nuclear heat generation term actually is the true energy since it is set at a fixed, predetermined value. Thus in this region, both the relative local error and the total global error are the same. In the coolant, however, the incoming heat flow itself can be incorrect, thus $e_{c}$ is only good for quantifying the relative local error. In order to understand the total error in the coolant, a global energy balance, $e_{g}$, is constructed to compare the nuclear heat generation against the change in coolant channel enthalpy

$$
\% e_{g}=\frac{\left|\Delta \dot{H}_{c}-\dot{E}_{p, g e n}\right|}{\dot{E}_{p, g e n}} \times 100 \%
$$

An additional error evaluation can be defined in the coupled model for the interdimensional model coupling mechanism itself. The error of the $3 \mathrm{D}$-to-2D coupling, $e_{i m c}$, can be calculated according to

$$
\% e_{i m c}=\frac{\left|\dot{E}_{c, \text { in }(\text { plate })}-\dot{E}_{p, \text { out }}\right|}{\dot{E}_{p, \text { out }}} \times 100 \% \text {. }
$$


The reverse, an evaluation of error in the $2 \mathrm{D}$-to-3D coupling, is not readily calculable. While the effects of the convective boundary condition in the fuel plate domain can be measured, there is no direct counterpart in the coolant domain with which to compare. The fact that the convective boundary is itself composed of values calculated in both domains makes any such error analysis all but impossible. Thus the 3D-to-2D error must be used to evaluate the entire model coupling mechanism.

Using $\dot{E}_{p, g e n}$ as a reference value, the energy terms used in calculating error can be normalized to produce

$$
\begin{gathered}
\frac{\dot{E}_{p, \text { gen }}}{\dot{E}_{p, \text { gen }}}=E_{p, \text { gen }}^{\prime}=1, \\
\frac{\dot{E}_{p, \text { out }}}{\dot{E}_{p, \text { gen }}}=E_{p, \text { out }}^{\prime}, \\
\frac{\dot{E}_{c, \text { in }(\text { plate })}}{\dot{E}_{p, \text { gen }}}=E_{c, \text { in }(\text { plate })}^{\prime},
\end{gathered}
$$

and

$$
\frac{\Delta \dot{H}_{c}}{\dot{E}_{p, g e n}}=\Delta H_{c}^{\prime}
$$

Another means of model evaluation lies in fuel plate temperatures. Specifically, the maximum fuel meat temperature can be used as a rough guide to determine a model's validity. While the energy balance method provides an empirical means of comparing a given model's solution against a known quantity, the maximum temperature method is only useful for providing a relative comparison between models. Thus when considering two models there is no way to declare one superior to the other based purely on their maximum fuel meat temperatures (assuming they are both within reason). In the course of a mesh convergence study in which many similar models are compared against each other, however, it can provide a meaningful way with which to track convergence.

\section{B. 3D Model Mesh Convergence}

Solutions presented in this section will be for the axially-consistent LEU fuel geometry only. The axially-graded LEU geometry is excluded sever hike in computational cost associated with solving the 
extra mesh elements in the axially-graded section of the fuel meat. Solutions are otherwise centered on the LEU, versus the HEU, geometry because the desire to model it was the initial impetus behind the thesis. The 3D model mesh convergence study is truncated in comparison to the coupled model's study. This is a result of the model's complexity and sensitivity. The axial mesh distribution was held constant for the mesh convergence study. The 2X MCNP axial mesh distribution was found to be adequate for all domains. Solutions for temperature along an axial cut line bisecting the point of maximum fuel meat temperature using both the $2 \mathrm{X}$ and $1 \mathrm{X}$ mesh distributions are shown in Fig. 7.1.

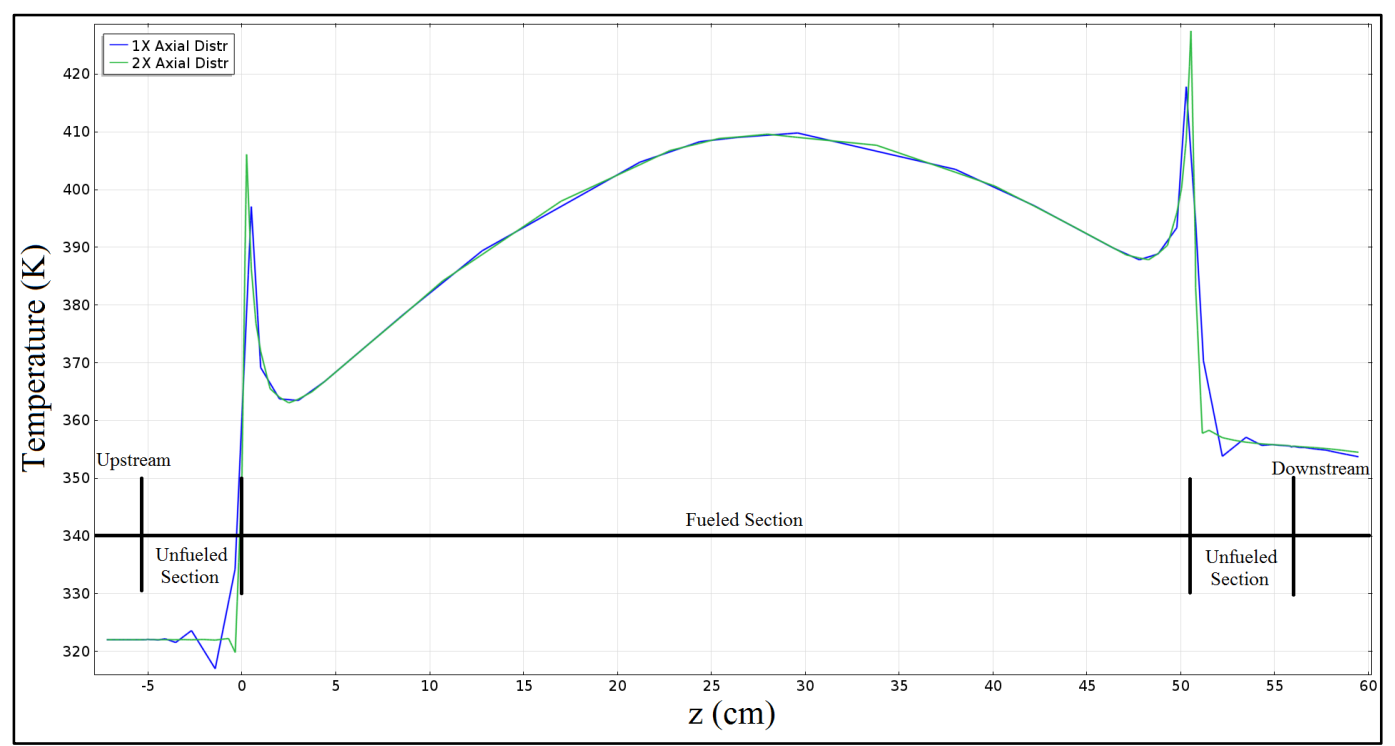

Fig. 7.1. Axial Cut Line Temperature Solution.

While the 1X MCNP axial mesh distribution clearly captures the overall contour of the temperature profile, it is insufficiently fine to accurately resolve abrupt shifts in the profile. The $2 \mathrm{X}$ distribution manages to resolve these shifts with greater clarity, especially with regards to the large temperature spikes at the top and bottom of the fuel meat. Additional refinements to the MCNP axial distribution could not be evaluated due to the increasing computational cost. Distribution refinements must necessarily double the previous distribution thus doubling the degrees of freedom in the model. With the $4 \mathrm{X}$ distribution the computational cost becomes too great to meet on available resources. It is doubtless that a $4 \mathrm{X}$ axial distribution would have further refined the profile in the vicinity of the temperature spikes. The agreement between the $1 \mathrm{X}$ and $2 \mathrm{X}$ distributions, combined with the diminishing returns to solution improvement that accompany mesh refinements, indicate that the $2 \mathrm{X}$ axial distribution can be assumed sufficient. 
With the axial mesh distribution fixed the mesh convergence study dealt only with altering mesh densities in the s-t plane. The breadth of the study was limited due to model solution instabilities at lower mesh resolutions and meshing difficulties at higher mesh resolutions. What results were able to be obtained, however, show good convergence towards a state of minimal energy balance error. The results of the 3D model mesh convergence study are shown in Table 7.1. Mesh $\mathrm{d} 2$ was selected as the ideal 3D model mesh against which to base comparisons. This mesh is representative of current core modeling efforts at HFIR using COMSOL. The fact that the observed maximum temperature changed by only 0.3 $\mathrm{K}$ between the two meshes indicates that any increase in mesh resolution, and thus solution quality, would affect very little change to the overall temperature profile of the model. Given that temperature is dropping with increases in mesh resolution, however, indicates that, if anything, the reported maximum temperature could be slightly higher than a true converged value.

\begin{tabular}{|c|c|c|c|c|c|c|c|c|}
\hline \multicolumn{2}{|c|}{ Table 7.1: 3D Model Mesh Convergence Study } \\
\hline Mesh & $\begin{array}{c}\text { Mesh Elements } \\
\text { (in thousands) }\end{array}$ & $E_{p, \text { gen }}^{\prime}$ & $E_{p, \text { out }}^{\prime}$ & $\Delta H_{c}^{\prime}$ & $e_{p}(\%)$ & $e_{c}(\%)$ & $e_{g}(\%)$ & $\begin{array}{c}\text { Max Fuel } \\
\text { Meat T [K] }\end{array}$ \\
\hline $\mathrm{d} 1$ & 1103 & 1.0000 & 1.0027 & 1.0021 & 0.267 & 0.060 & 0.207 & 432.1 \\
\hline $\mathrm{d} 2$ & 2311 & 1.0000 & 0.9997 & 0.9995 & 0.034 & 0.019 & 0.053 & 431.8 \\
\hline
\end{tabular}

\section{Coupled Model Mesh Convergence}

The separation of the coupled model into two distinct domains permits a more detailed mesh convergence study to be performed. In fact, it permits two mesh convergence studies to be performedone for the coolant and one for the fuel plate. Using the previously defined local error quantities, this permits the mesh in each domain to be optimized individually, thus ensuring adequate mesh resolution to capture physics while precluding the risk of unnecessary refinement. The coolant domain mesh convergence was performed first. For that, the fuel plate domain mesh was held constant and the coolant domain successively refined until a mesh-converged solution was achieved. The fuel mesh chosen was of a sufficient resolution that $e_{p}$ equaled zero for all cases. Similarly, $e_{i m c}$ was found to be zero at all times, not just in the coolant mesh convergence study but in every solution obtained over the duration of thesisrelated work. Thus it can be said that

$$
\% e_{i m c}=0
$$


is true at all times. Coolant meshes were judged based on $e_{c}$ which, with $e_{p}=e_{i m c}=0$, equals $e_{g}$ in these cases. The results of the coolant mesh convergence study are shown in Table 7.2. The data in columns four, five, and six are plotted in Figs. 7.2, 7.3, and 7.4, respectively, as functions of the number of coolant mesh elements in each model. The plot of $e_{c}$ is presented in logarithmic format to demonstrate that the error is decreasing near-logarithmically in accordance with finite element analysis theory.

\begin{tabular}{|c|c|c|c|c|c|}
\hline \multicolumn{5}{|c|}{ Table 7.2: Coupled Model Coolant Domain Mesh Convergence Study } \\
\hline Mesh & $\begin{array}{c}\text { Coolant Mesh Elements } \\
\text { (in thousands) }\end{array}$ & $E_{c, \text { in (plate) }}^{\prime}$ & $\Delta H_{c}^{\prime}$ & $e_{c}(\%)$ & $\begin{array}{c}\text { Max Fuel } \\
\text { Meat T (K) }\end{array}$ \\
\hline $\mathrm{c} 1$ & 163 & 1.0000 & 0.8493 & 15.07 & 425.1 \\
\hline $\mathrm{c} 2$ & 301 & 1.0000 & 0.9301 & 6.99 & 427.2 \\
\hline $\mathrm{c} 3$ & 456 & 1.0000 & 0.9686 & 3.14 & 428.2 \\
\hline $\mathrm{c} 4$ & 717 & 1.0000 & 0.9900 & 1.00 & 428.7 \\
\hline $\mathrm{c} 5$ & 1,035 & 1.0000 & 0.9971 & 0.29 & 428.9 \\
\hline $\mathrm{c} 6$ & 1,429 & 1.0000 & 1.0006 & 0.06 & 429.0 \\
\hline
\end{tabular}

*Fuel plate domain mesh elements (in thousands) $=454$

$* *$ Residual tolerance $=1.00 \times 10^{-2}$

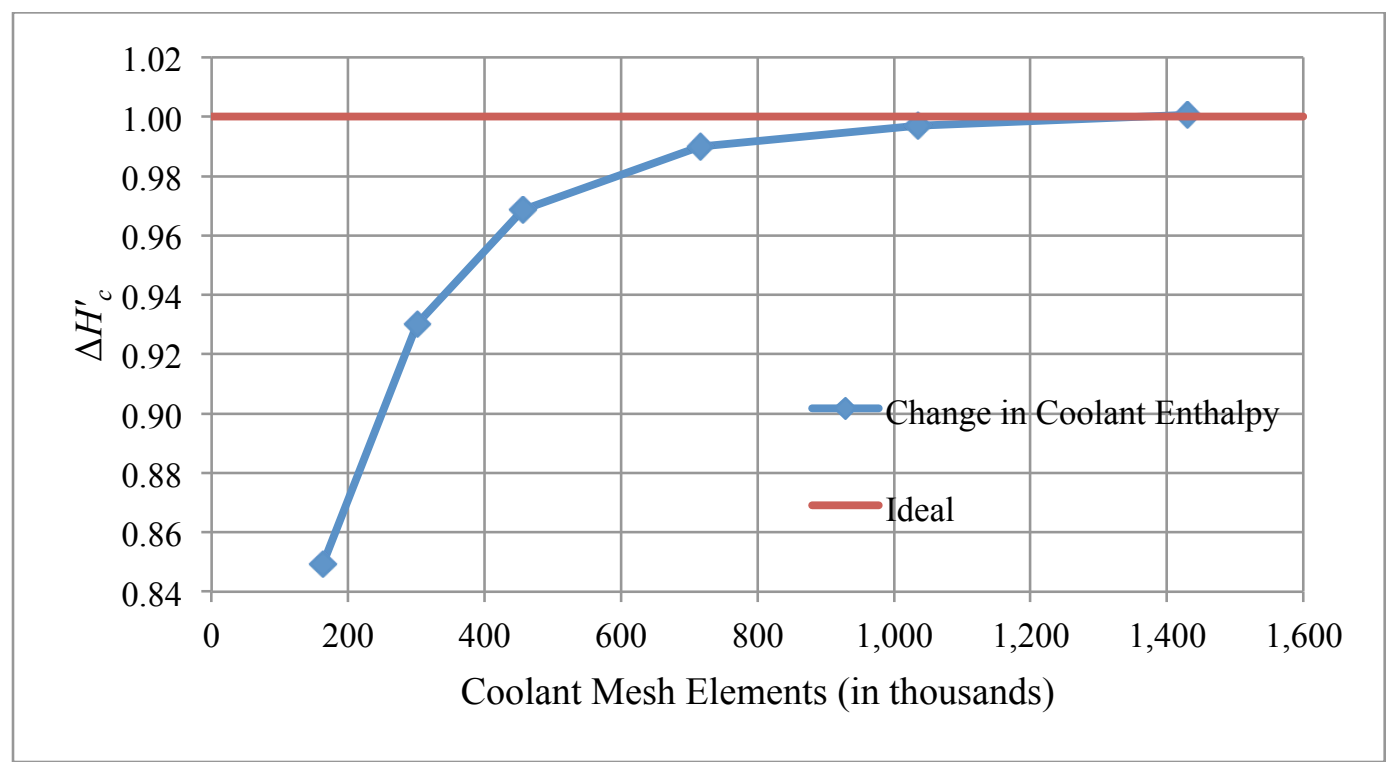

Fig. 7.2. Coupled Model Coolant Mesh Convergence-Coolant Channel Enthalpy. The normalized nuclear heat generation, 1.00, is marked with a red line to show the coolant channel's convergence towards the correct value. 


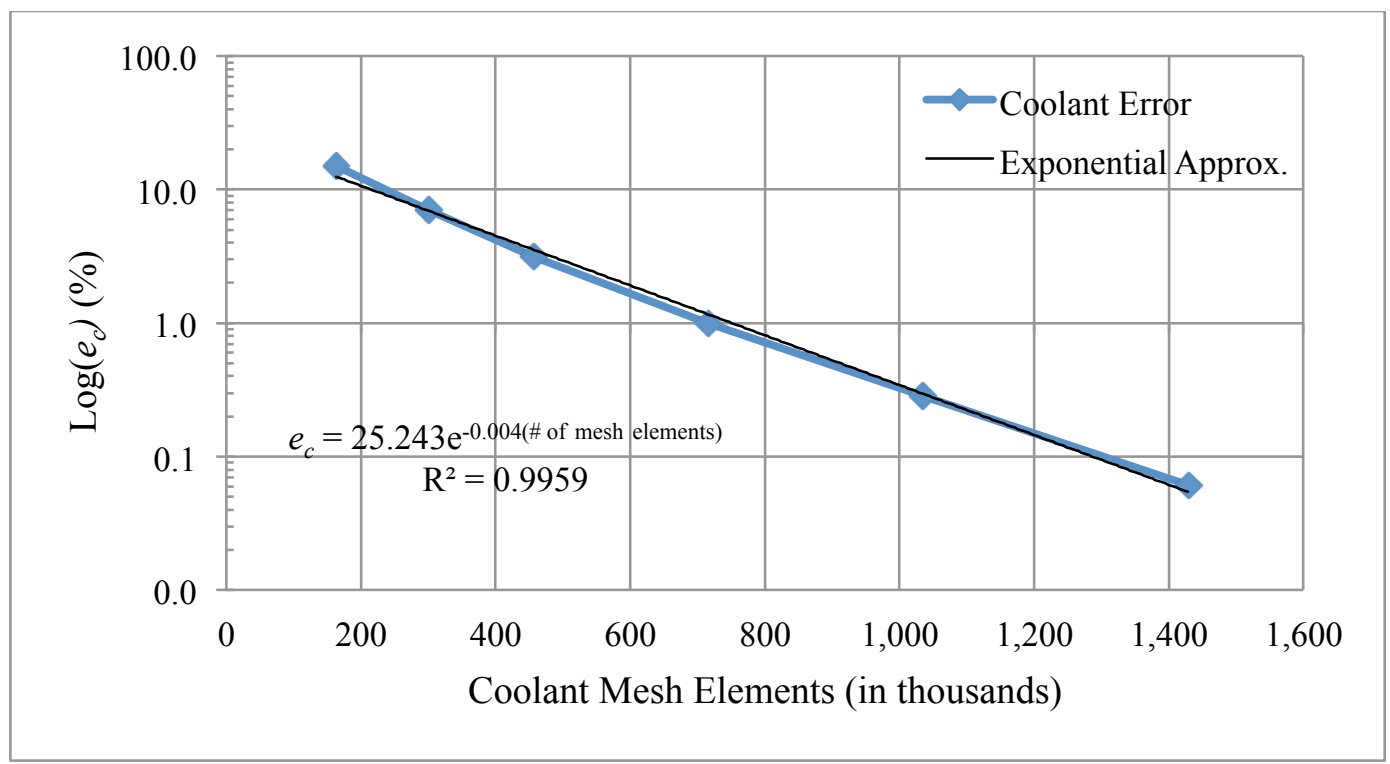

Fig. 7.3. Coupled Model Coolant Mesh Convergence_Logarithmic Coolant Error.

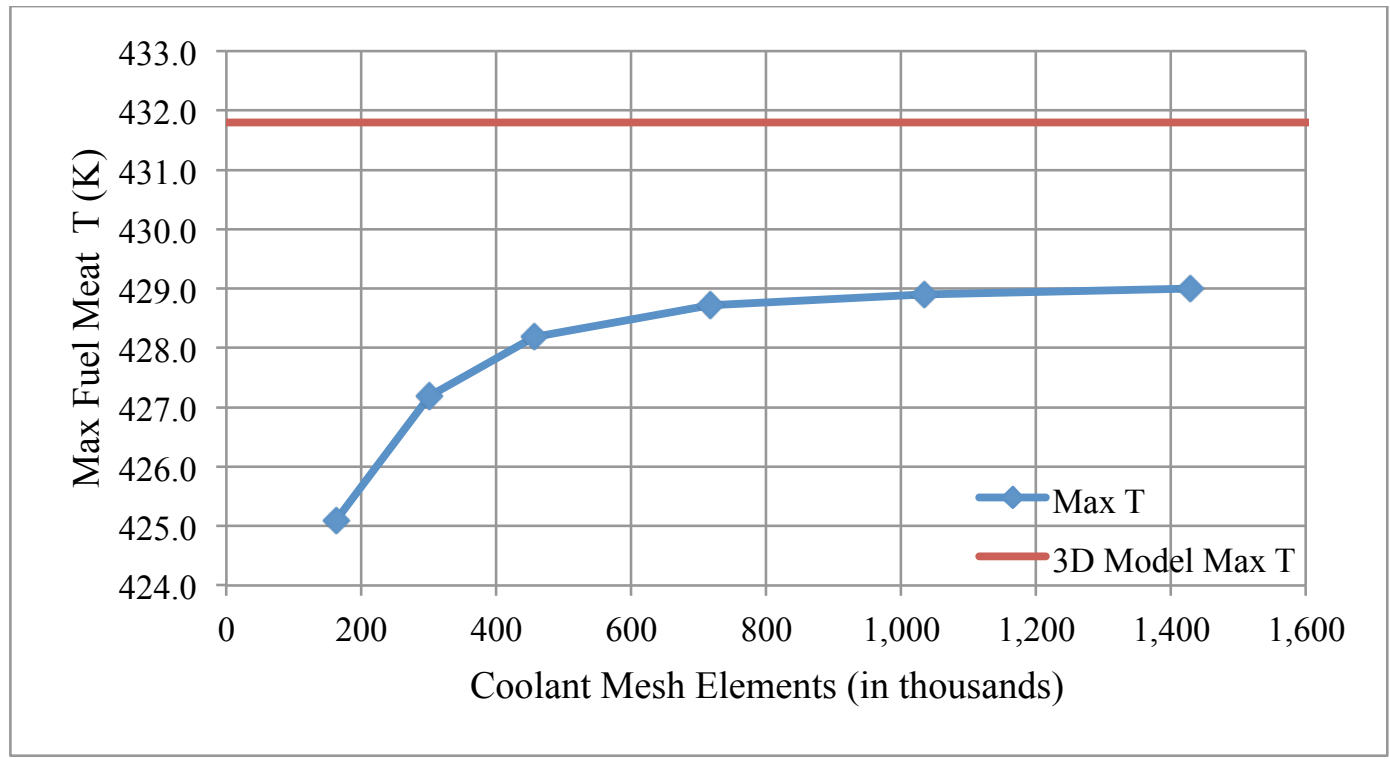

Fig. 7.4. Coupled Model Coolant Mesh Convergence-Max Fuel Meat Temperature. The $3 \mathrm{D}$ model's maximum fuel meat temperature is marked with a red line to demonstrate the offset between the two models' converged states.

The coolant mesh convergence study shows not only good convergence but also that the model is converging to a state very similar to that of the converged 3D model. The converged temperature is only $2.8 \mathrm{~K}$ below that of the 3D model. With the goal of balancing the efficiency and accuracy of the model, a total error of $e_{g} \approx 5 \%$ was chosen as an acceptable margin. Given the relative difficulty of modeling the 
RANS equations in the coolant versus modeling heat transfer in the fuel plate, the majority of this error should be expected to come from the coolant. Thus mesh c3 was chosen as the ideal coolant mesh resolution. With only 456,000 mesh elements it has one-third the number of mesh elements as the "converged" mesh. Even still it has an energy balance error of only 3\% and its maximum fuel meat temperature is still within $1 \mathrm{~K}$ of the converged value.

With the coolant mesh held constant at mesh c3, a mesh convergence study was then performed on the fuel plate domain. All meshes considered utilized quadratic basis functions. The results of the fuel plate mesh convergence study are shown in Table 7.3. The data in columns six, eight, and nine are plotted in Figs. 7.5, 7.6, and 7.7, respectively.

\begin{tabular}{|c|c|c|c|c|c|c|c|c|}
\hline \multicolumn{7}{|c|}{ Table 7.3: Coupled Model Fuel Plate Domain Mesh Convergence Study } \\
\hline Mesh & $\begin{array}{c}\text { Fuel Plate Mesh } \\
\text { Elements } \\
\text { (in thousands) }\end{array}$ & $E_{p, \text { gen }}^{\prime}$ & $E_{p, \text { out }}^{\prime}$ & $\Delta H_{c}^{\prime}$ & $e_{p}(\%)$ & $e_{c}(\%)$ & $e_{g}(\%)$ & $\begin{array}{c}\text { Max Fuel } \\
\text { Meat T } \\
(\mathrm{K})\end{array}$ \\
\hline $\mathrm{p} 1$ & 24 & 1.0000 & 1.0036 & 0.9665 & 0.356 & 3.689 & 3.346 & 426.9 \\
\hline $\mathrm{p} 2$ & 35 & 1.0000 & 1.0003 & 0.9676 & 0.033 & 3.269 & 3.237 & 427.6 \\
\hline $\mathrm{p} 3$ & 51 & 1.0000 & 1.0002 & 0.9681 & 0.020 & 3.209 & 3.190 & 427.9 \\
\hline $\mathrm{p} 4$ & 84 & 1.0000 & 1.0002 & 0.9682 & 0.016 & 3.195 & 3.180 & 428.2 \\
\hline $\mathrm{p} 5$ & 110 & 1.0000 & 1.0001 & 0.9683 & 0.009 & 3.177 & 3.168 & 428.1 \\
\hline $\mathrm{p} 6(\mathrm{c} 3)$ & 454 & 1.0000 & 1.0001 & 0.9686 & 0.008 & 3.146 & 3.138 & 428.2 \\
\hline
\end{tabular}

${ }^{*}$ Coolant channel domain mesh elements (in thousands) $=456$

$* *$ Residual tolerance $=1.00 \times 10^{-2}$

The convergence data for the fuel plate mesh study does not follow the same orderly exponential convergence rate that the coolant mesh study did. In looking at the fuel plate error for cases p6 and p5 it is apparent that the study reaches machine accuracy prior to case $\mathrm{p} 6$. The difference in solution in case $\mathrm{p} 6$ is attributable only to round-off error. 


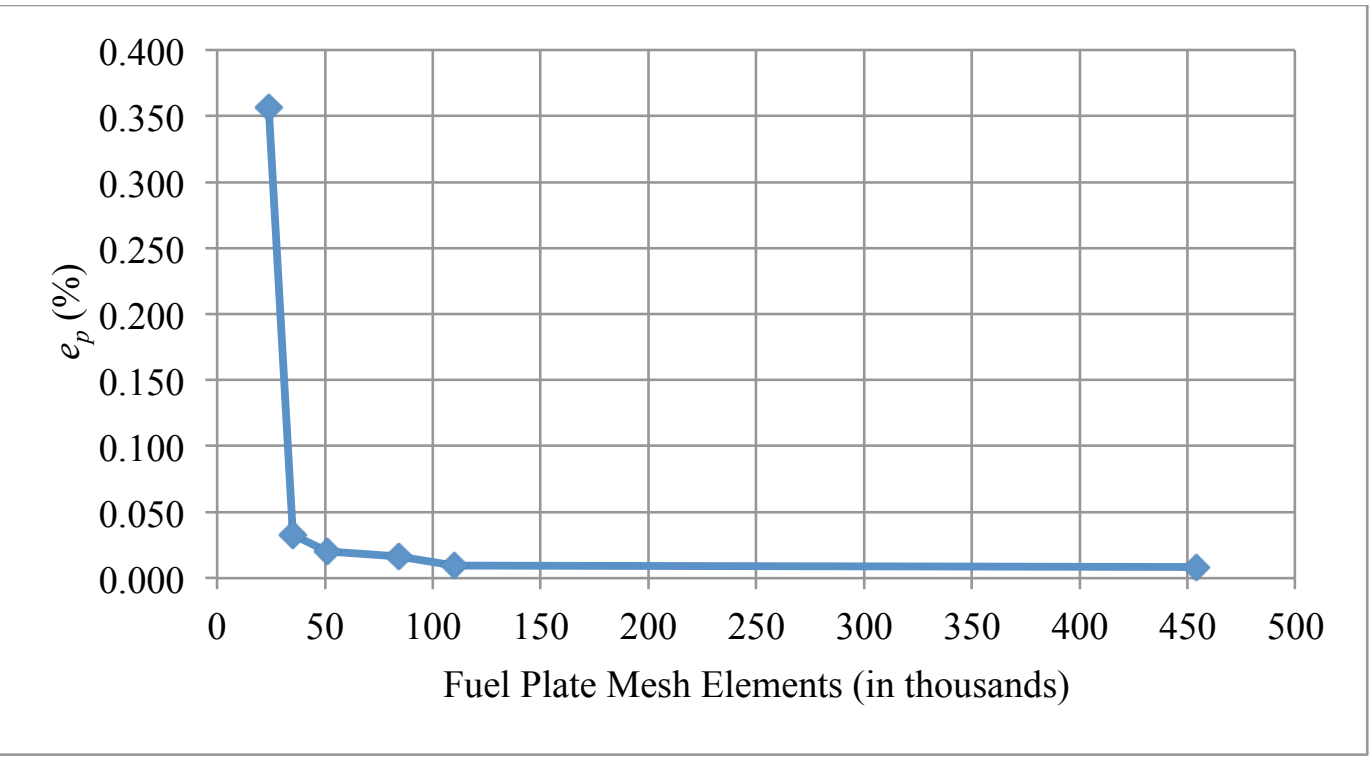

Fig. 7.5. Coupled Model Fuel Plate Mesh Convergence_Fuel Plate Error.

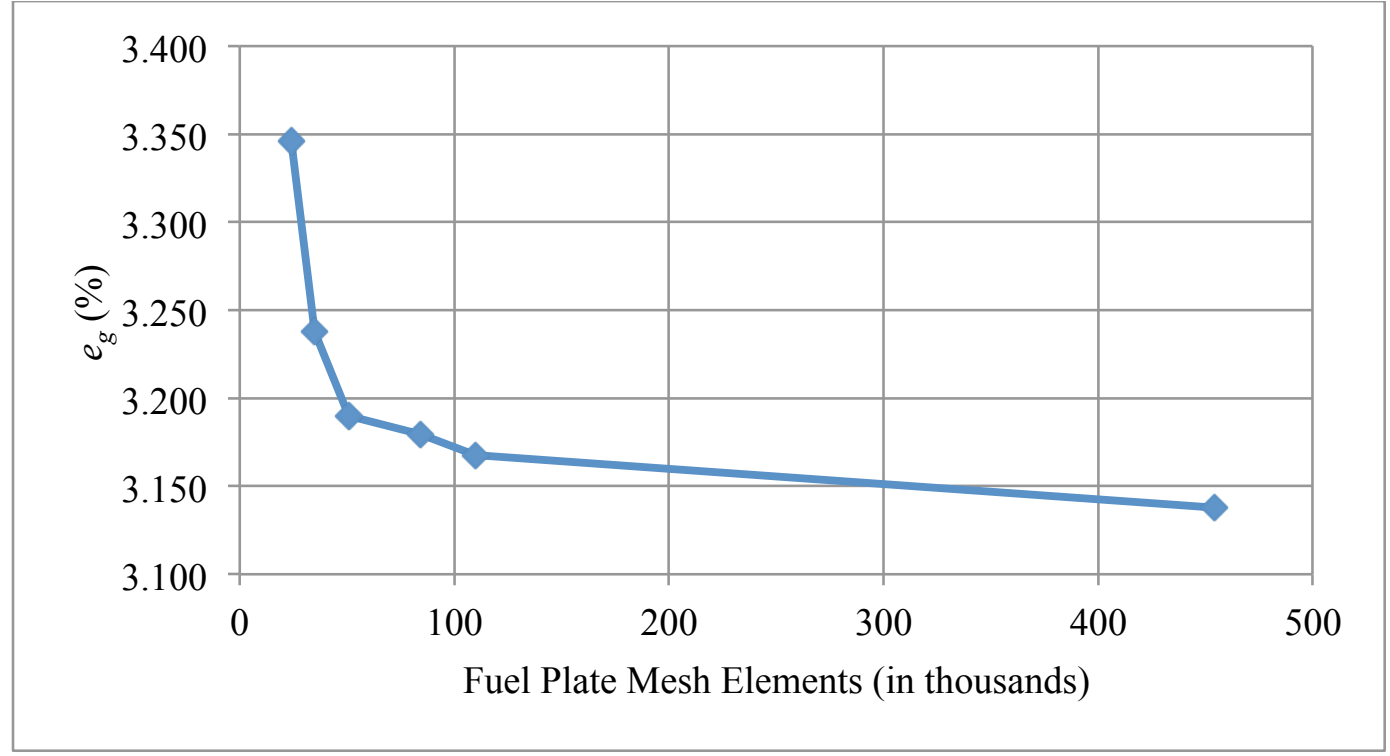

Fig. 7.6. Coupled Model Fuel Plate Mesh Convergence_Global Error.

The fuel plate domain stays in a near-constant state, at least from the perspective of the energy balance, from mesh p2 to mesh p6. It is only once the mesh count passes below 35,000 that the local fuel plate error, $e_{p}$, begins to increase drastically. Even then, "drastically" amounts to a $0.3 \%$ increase in local error. The local energy balance data would indicate that the mesh convergence study was focused on over-refined meshes in which increases to mesh resolution do not yield commensurate increases in 
solution quality. Mesh p1 is rapidly approaching the coarsest possible mesh. As shown in Fig. 7.8, mesh p1 already spans the width of the fuel meat with only two mesh elements and, in some places, the surrounding fuel plate with only one. Further mesh reduction could be achieved only by decreasing the mesh resolution along the span of the plate.

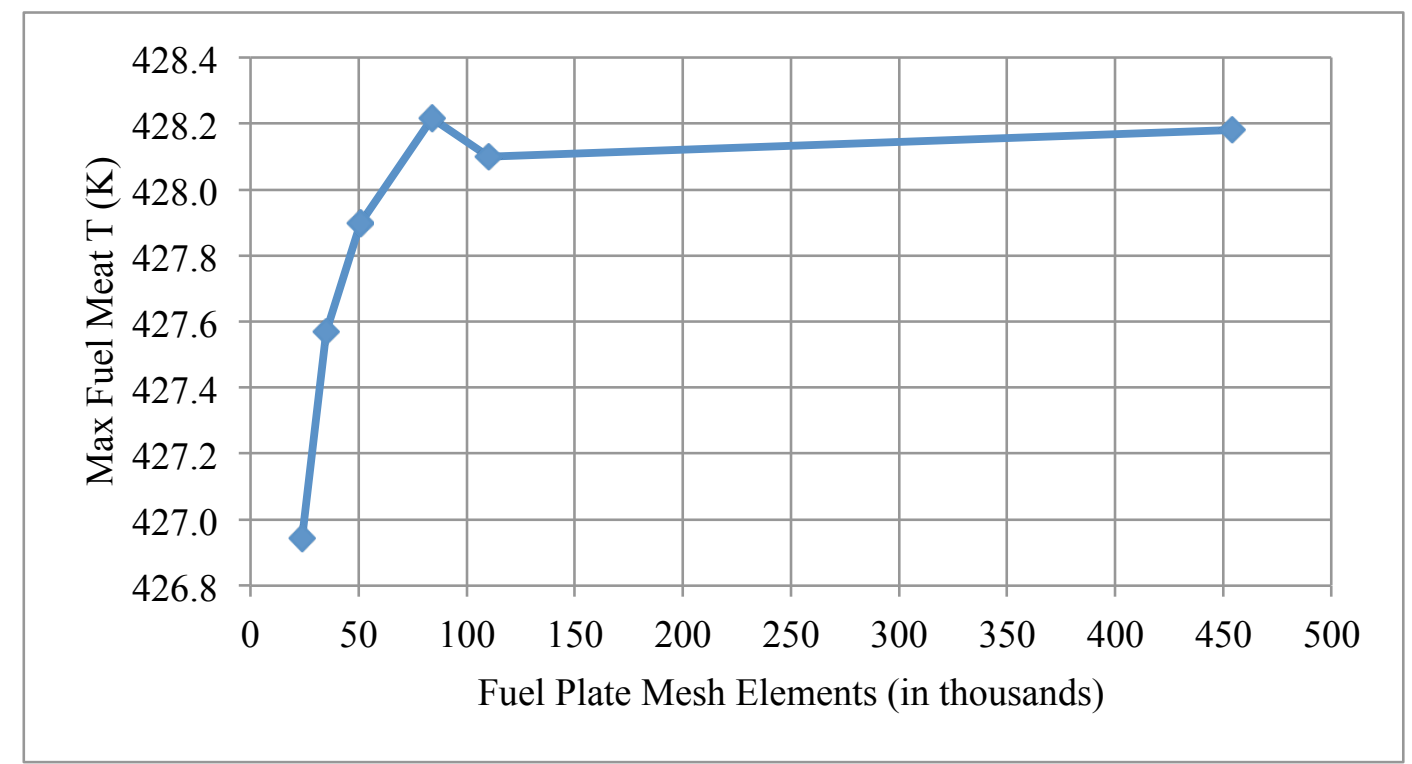

Fig. 7.7. Coupled Model Fuel Plate Mesh Convergence—Max Fuel Meat Temperature.

The global error data suggests that further mesh reduction is unnecessary. Its convergence shows a steady increase on return with decreasing mesh resolution throughout the study. The same is true of the maximum fuel meat temperature data which increases rapidly between meshes $\mathrm{p} 1$ and $\mathrm{p} 4$ before leveling out. In analyzing the global error for the fuel plate mesh convergence study it is apparent that the imposed 5\% limit was never reached. The maximum fuel meat temperature becomes the limiting factor instead due its higher sensitivity to fuel plate mesh resolution. An ad hoc temperature "error" can be formulated using the maximum fuel meat temperature from mesh $\mathrm{d} 2$ as the ideal. This difference, $e_{T}$, is given by

$$
\% e_{T}=\left|\frac{T_{\max }-T_{\max , \mathrm{d} 2}}{T_{\max , \mathrm{d} 3}-273.15}\right| \times 100 \%
$$

where all temperature values are in Kelvin. Note that the temperature "error" value is not a true error as the reference value, $T_{\max } \mathrm{d} 2$, is not known to be correct. It is merely a reference value with which to compare deviations in subsequent model variants. The plot of temperature error is shown in Fig. 7.9. 


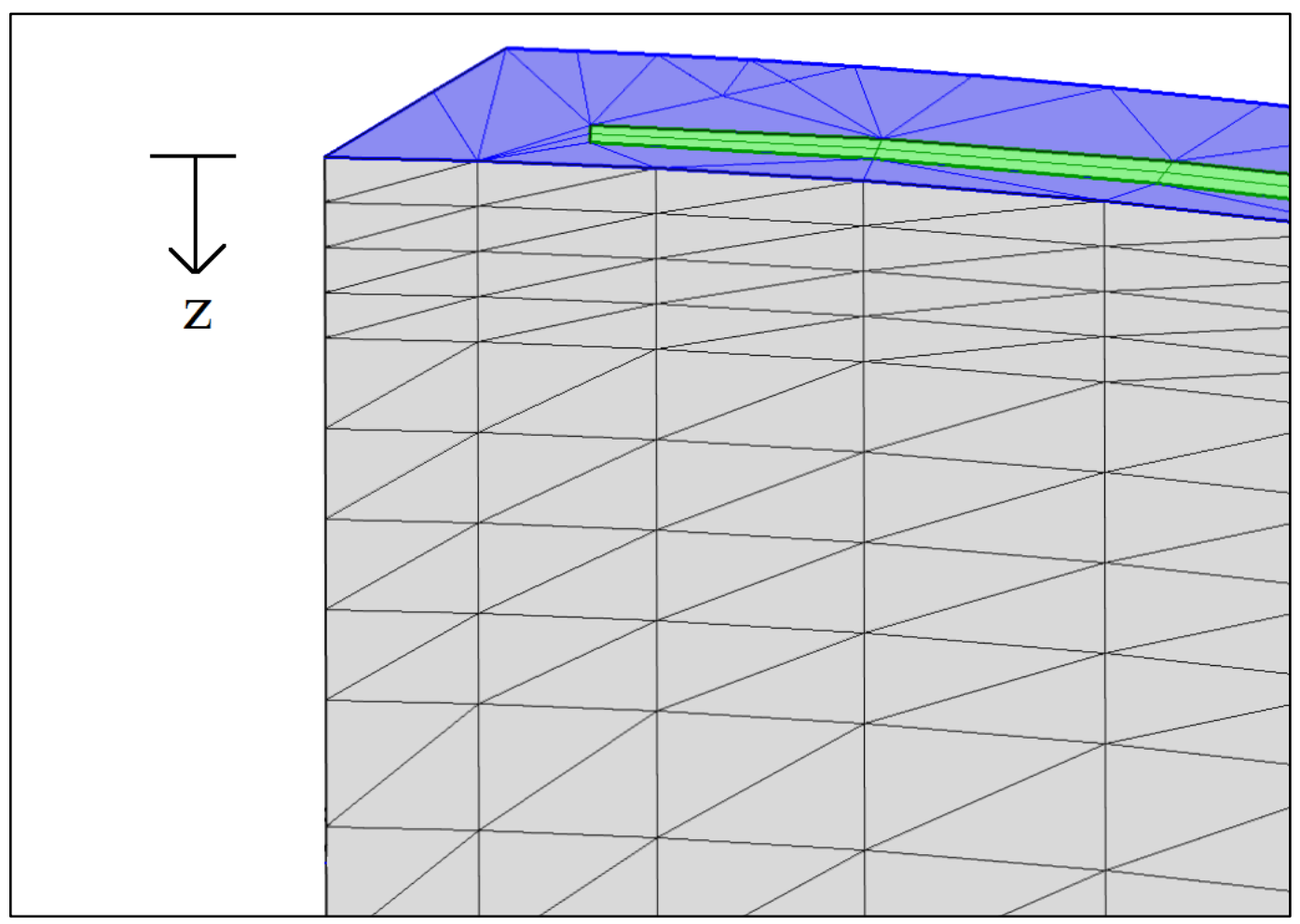

Fig. 7.8. Coupled Model Fuel Plate Mesh p1. Fuel plate $s-t$ face is highlighted in blue, fuel meat face in green.

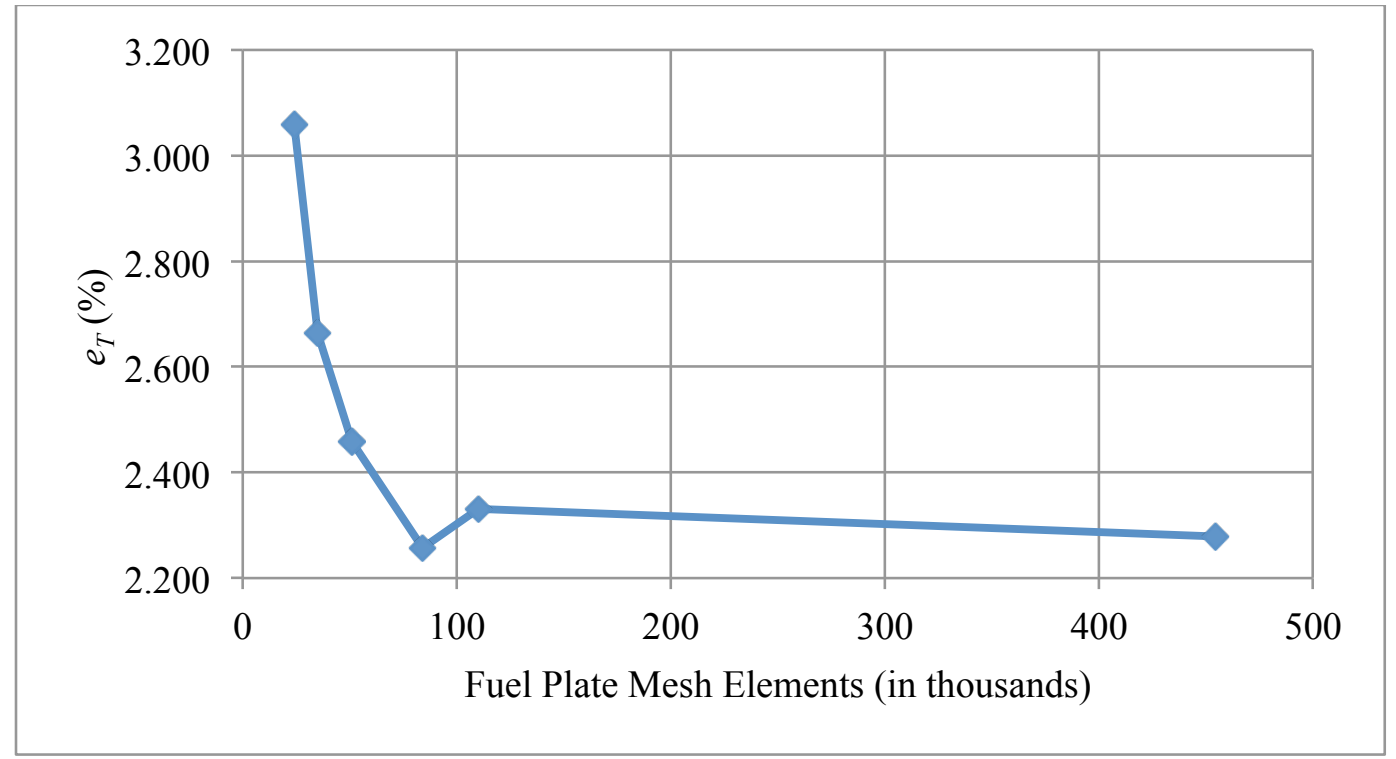

Fig. 7.9. Coupled Model Fuel Plate Mesh Convergence-Max Fuel Meat T Error. 
Mesh p3, with a maximum temperature of $427.9 \mathrm{~K}$ and a temperature error of $2.46 \%$, was chosen as the ideal mesh. With ideal meshes selected for each domain and associated solution characteristics with which to evaluate alterations to the configuration, attention was turned once again to the coolant domain. Rather than seeking to alter the solution through either mesh reduction or refinement, simplifying mesh alternatives were sought that affected the solution as little as possible. Such meshes were variants of the constant-width extruded mesh detailed in Section V.

The results of the alternate coolant mesh study are shown in Table 7.4. The constant-width mesh distributions, meshes alt1-alt6, showed promising results. Meshes of this type with sufficient axial distributions allowed the overall number of mesh elements in the coolant to be greatly decreased without a serious effect on the solution. Mesh alt7 was included only to show that with sufficient mesh resolution the constant-width structured meshes converged to a sufficiently small global error. Interestingly, the $2 \mathrm{X}$ MCNP distribution, mesh alt9, failed to produce good results. The reason for this is unclear. Its use in the 3D model, as well as the fuel plate domain of the coupled model, has no ill effect on the solution. Its use in the coupled coolant domain, however, causes the model to converge to a much hotter state. The data for meshes alt1-alt6 from columns 8 and 9 are plotted in Figs. 7.10 and 7.11, respectively. Mesh alt7 was excluded because it skewed the scale of the convergence plots, making the inter-mesh behavior between coarser meshes harder to analyze. Reference quantities for meshes $\mathrm{p} 3$ and $\mathrm{d} 2$ are included where appropriate.

\begin{tabular}{|c|c|c|c|c|c|c|c|c|}
\hline \multicolumn{7}{|c|}{ Table 7.4: Coupled Model Alternate Coolant Mesh Study } \\
\hline Mesh & $\begin{array}{c}\text { Coolant Mesh } \\
\text { Elements } \\
\text { (in thousands) }\end{array}$ & $\begin{array}{c}\text { Structured } \\
\text { Mesh? }\end{array}$ & $\begin{array}{c}\text { Axial } \\
\text { Mesh } \\
\text { Distr }\end{array}$ & $E_{p, \text { out }}^{\prime}$ & $\Delta H_{c}^{\prime}$ & $e_{c}(\%)$ & $e_{g}(\%)$ & $\begin{array}{c}\text { Max Fuel } \\
\text { Meat T (K) }\end{array}$ \\
\hline alt1 & 12 & $\mathrm{Y}$ & $l / 50$ & 1.0002 & 1.0253 & 2.512 & 2.533 & 429.3 \\
\hline alt2 & 15 & $\mathrm{Y}$ & $l / 100$ & 1.0002 & 1.0351 & 3.488 & 3.509 & 429.6 \\
\hline alt3 & 21 & $\mathrm{Y}$ & $l / 200$ & 1.0002 & 1.0392 & 3.904 & 3.925 & 429.7 \\
\hline alt4 & 28 & $\mathrm{Y}$ & $l / 300$ & 1.0002 & 1.0389 & 3.870 & 3.890 & 429.7 \\
\hline alt5 & 46 & $\mathrm{Y}$ & $l / 600$ & 1.0002 & 1.0374 & 3.718 & 3.739 & 429.7 \\
\hline alt6 & 71 & $\mathrm{Y}$ & $l / 1000$ & 1.0002 & 1.0341 & 3.385 & 3.406 & 429.6 \\
\hline alt7 & 319 & $\mathrm{Y}$ & $l / 5000$ & 1.0002 & 1.0111 & 1.086 & 1.106 & 429.1 \\
\hline alt8 (p3) & 456 & $\mathrm{~N}$ & $\mathrm{~N} / \mathrm{A}$ & 1.0002 & 0.9681 & 3.209 & 3.190 & 427.9 \\
\hline alt9 & 14 & $\mathrm{Y}$ & 2X MCNP & 1.0002 & 1.5189 & 51.860 & 51.891 & 442.8 \\
\hline
\end{tabular}

*Constant-width axial mesh distributions are given as functions of plate length, $l$, divided by number of axial elements.

$* *$ Fuel plate domain mesh elements (in thousands) $=454$

$* * *$ Residual tolerance $=1.00 \times 10^{-2}$ 


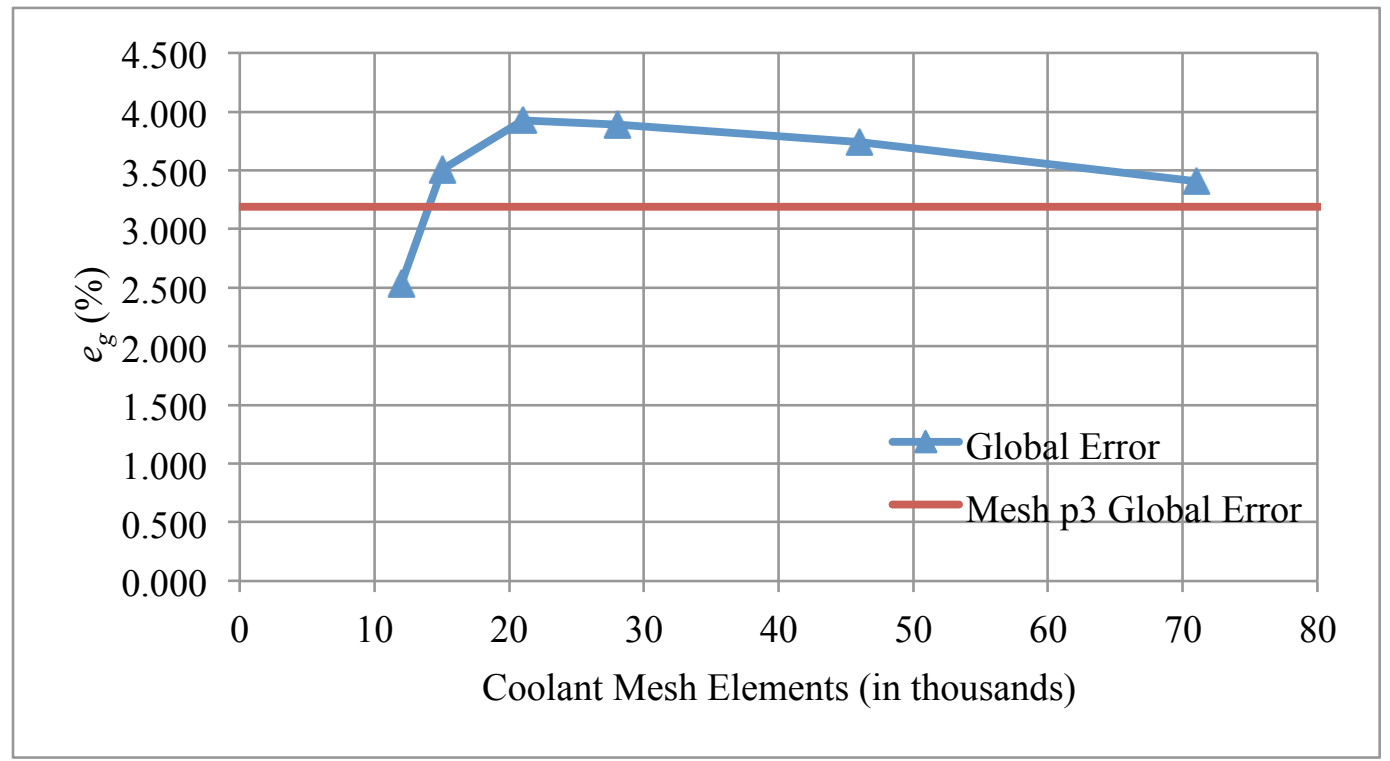

Fig. 7.10. Coupled Model Alternate Coolant Mesh Study_Global Error.

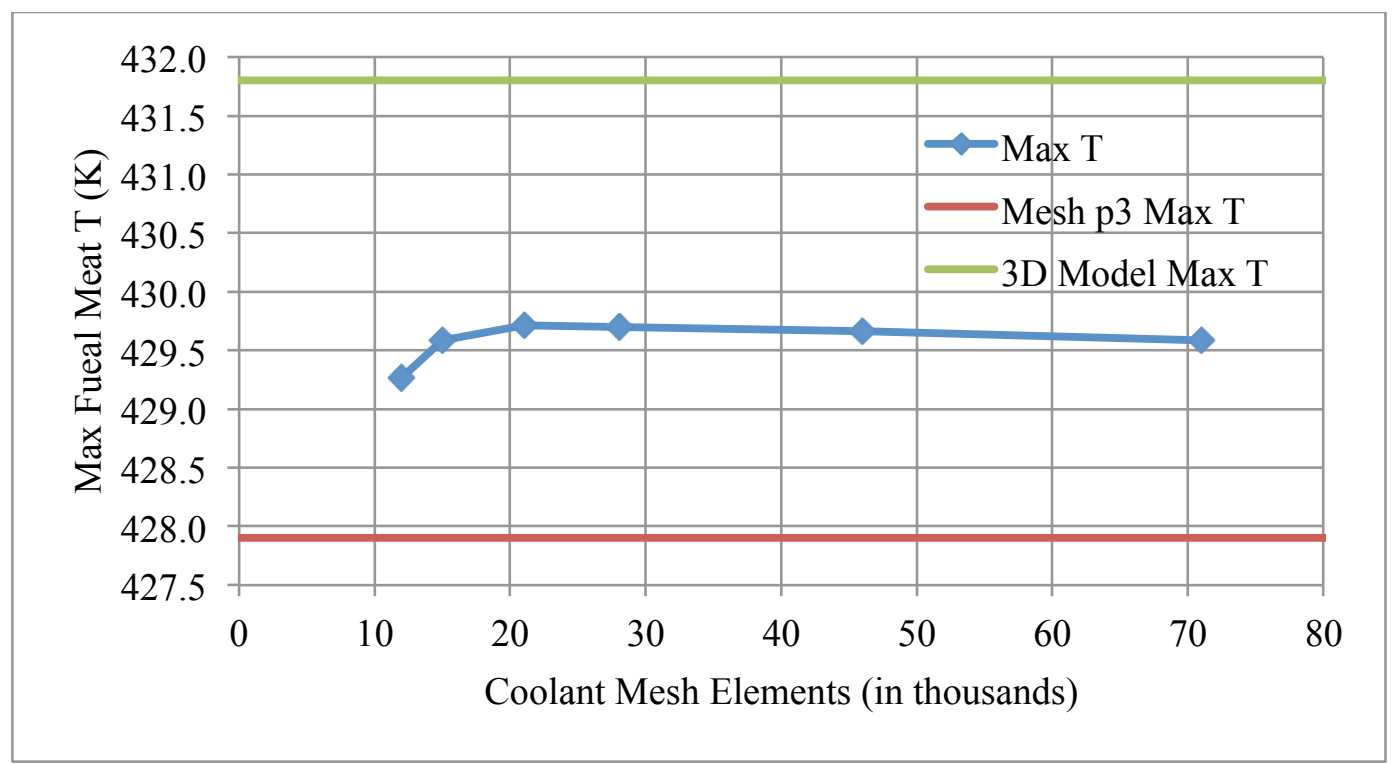

Fig. 7.11. Coupled Model Alternate Coolant Mesh Study—Max Fuel Meat T.

The fuel plate error, $e_{p}$, is constant at $0.02 \%$ for each case and was thus not plotted. The coolant error, $e_{c}$, is offset from the global error by this amount making the two nearly identical. From the temperature plot it is clear that the alternate coolant mesh variant of the coupled model is converging to a state 
somewhere between the 3D model and the initial converged coupled model. In both plots it is clear that meshes alt 3 through alt 6 show solid convergence while meshes alt 1 and alt 2 begin to diverge significantly with decreases to mesh resolution. The fact that these coarse meshes momentarily reproduce a model state close to the converged state is not to be taken as a positive attribute but merely a coincidence that rather than continuing to diverge upwards they change direction and diverge towards a lower energy state.

Interestingly, meshes alt3 through alt6 are approach a converged state from higher energy states. The opposite was true with the original unstructured coolant mesh in which both temperature and coolant channel enthalpy change approached their respective values from below. This behavior, coupled with the fact that the alternate coolant mesh model is converging towards a higher state than the original model, reintroduces a small measure of conservatism into the model that was removed, dually, by the smoothing effects of the coolant channel dimensional reduction and the convergence characteristics of the original coupled model.

In looking at the global energy balance errors it is clear that mesh alt3 is the coarsest alternate coolant mesh to sufficiently approximate the original converged solution. The fact that its maximum temperature is also closest to the 3D model's maximum temperature, while appealing, is really arbitrary in this instance. The only meaningful comparison is to the alternate model's converged temperature which mesh alt3 does a sufficient job of approximating.

One final tweak is made to this model. The alternate mesh study was performed using constant-width cells throughout the length of the fuel plate section of the channel. While this facilitated a faster mesh study it also created a large mesh size disparity on the mesh boundary separating the structured mesh from the unstructured mesh upstream and downstream of the fuel plate. In order to smooth over this transition, the first and last structured mesh elements in mesh alt3 were replaced with successively smaller transition mesh elements as shown in Fig. 7.12. This has a minimal, yet positive, effect on the solution as shown in Table 7.5 . 


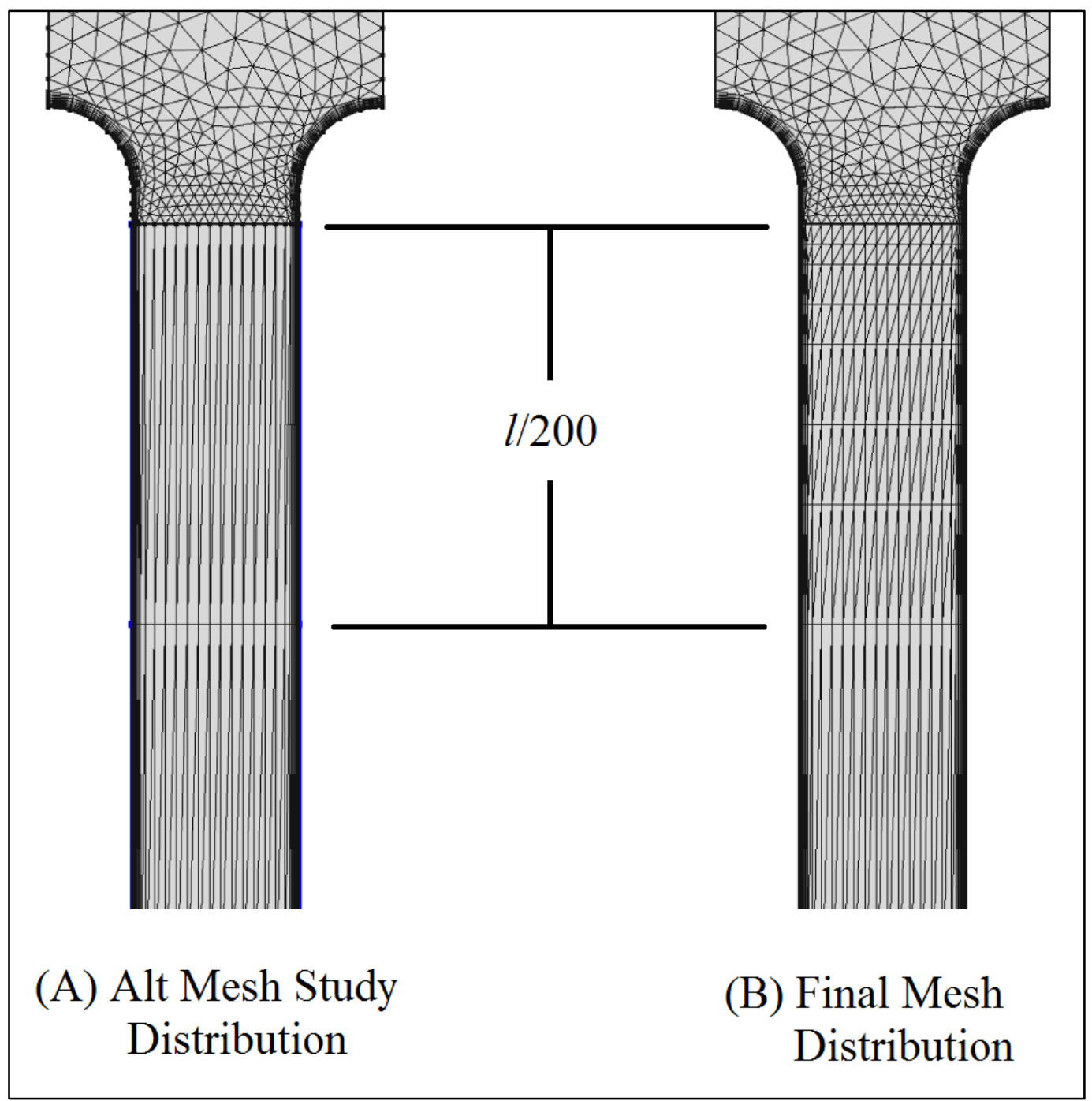

Fig. 7.12. Mesh alt3 Distribution Comparison.

\begin{tabular}{|c|c|c|c|c|c|c|c|c|}
\hline \multicolumn{9}{|c|}{ Table 7.5: Mesh alt3 Comparison Solutions } \\
\hline Mesh & $\begin{array}{c}\text { Coolant Mesh } \\
\text { Elements } \\
\text { (in thousands) }\end{array}$ & $\begin{array}{c}\text { Structured } \\
\text { Mesh? }\end{array}$ & $\begin{array}{c}\text { Axial Mesh } \\
\text { Distribution }\end{array}$ & $E_{p, \text { out }}^{\prime}$ & $\Delta H_{c}^{\prime}$ & $e_{c}(\%)$ & $e_{g}(\%)$ & $\begin{array}{c}\text { Max Fuel } \\
\text { Meat T }[\mathrm{K}]\end{array}$ \\
\hline alt3 & 21 & $\mathrm{Y}$ & $l / 200$ & 1.0002 & 1.0392 & 3.904 & 3.925 & 429.7 \\
\hline alt3b & 22 & $\mathrm{Y}$ & $\sim l / 200$ & 1.0002 & 1.0358 & 3.557 & 3.577 & 429.6 \\
\hline
\end{tabular}

\section{Validation of Simplifying Coupled Model Assumptions}

As mentioned previously, the exclusion of the side plates from the coupled model is due in large part to their incompatibility with the $2 \mathrm{D}$ coolant domain. Their exclusion is justified by results from the $3 \mathrm{D}$ 
model which show that heat flow into the side plates is minimal compared to that in the fuel plate and coolant channel. Local heat flux into the inner side plate is shown in Fig. 7.13. Across the boundary with the fuel plate heat flow is predominantly into the side plate. Across the boundary with the coolant channel heat flow is predominantly outward. A surface integral of heat flux through the fuel plate boundary shows that the total inward heat flow is only $578 \mathrm{~W}$. The total outward heat flow through the coolant common boundary is only $227 \mathrm{~W}$. Both of these are three orders of magnitude smaller than the total nuclear heat generation production. It is worth noting that replacing the thermally insulated boundary on the outer walls of the side plates in the 3D model would most likely increase these heat flow values significantly. Since this was not accounted for in the 3D model, however, the effect on the solution from removing them in the coupled model is minimal

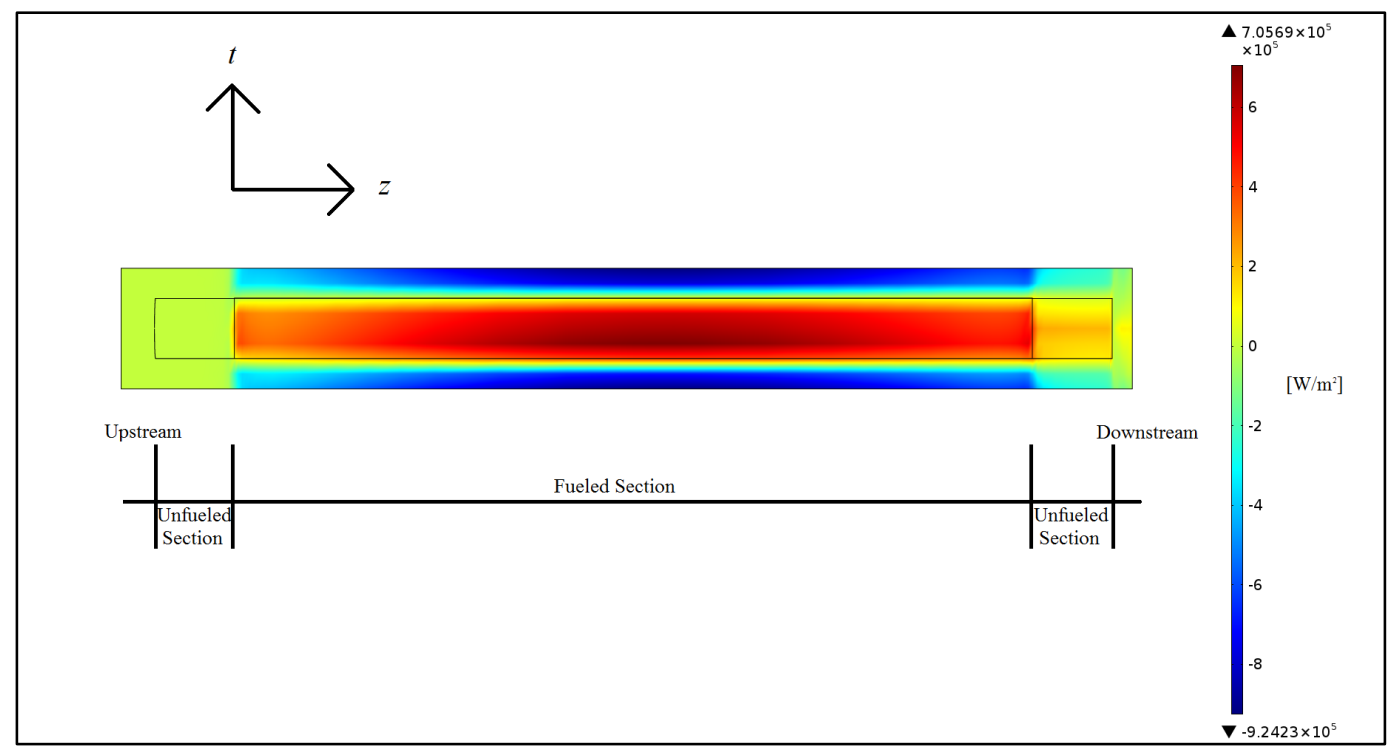

Fig. 7.13. 3D Model Inner Side Plate Inward Heat Flux. Figure is not to scale. The $t$-direction has been stretched by a factor of 20 for ease of viewing. The fuel plate is outlined in three parts with the coolant channel surrounding it.

Additional simplifications can be made to the coupled model through observation of preliminary solutions to the model itself. The use of a single bulk velocity value along the length of the plate is validated by the short length of the hydrodynamic entrance region, as shown in Fig. 7.14. For reference, the leading edge of the fuel plate is situated at $-5.08 \mathrm{~cm}$. Within $2 \mathrm{~cm}$, and still well before the top of the fuel section, the flow is fully developed. Similarly, the onset of turbulence is extremely rapid as shown in Fig. 7.15, validating both the assumption of turbulent flow throughout as well as verifying that the model 
remains within the limits of the modified Hausen correlation as experimentally verified by Gambill and Bundy. The thermal entrance region, shown in in Fig. 7.16, is also extremely short. The onset of thermally developed flow is delayed until just upstream of the fueled section. This gives the flow adequate space to become both fully developed and fully turbulent before interacting significantly with the fuel. The distance from onset to fully developed thermal flow occurs in less than $4 \mathrm{~cm}$.

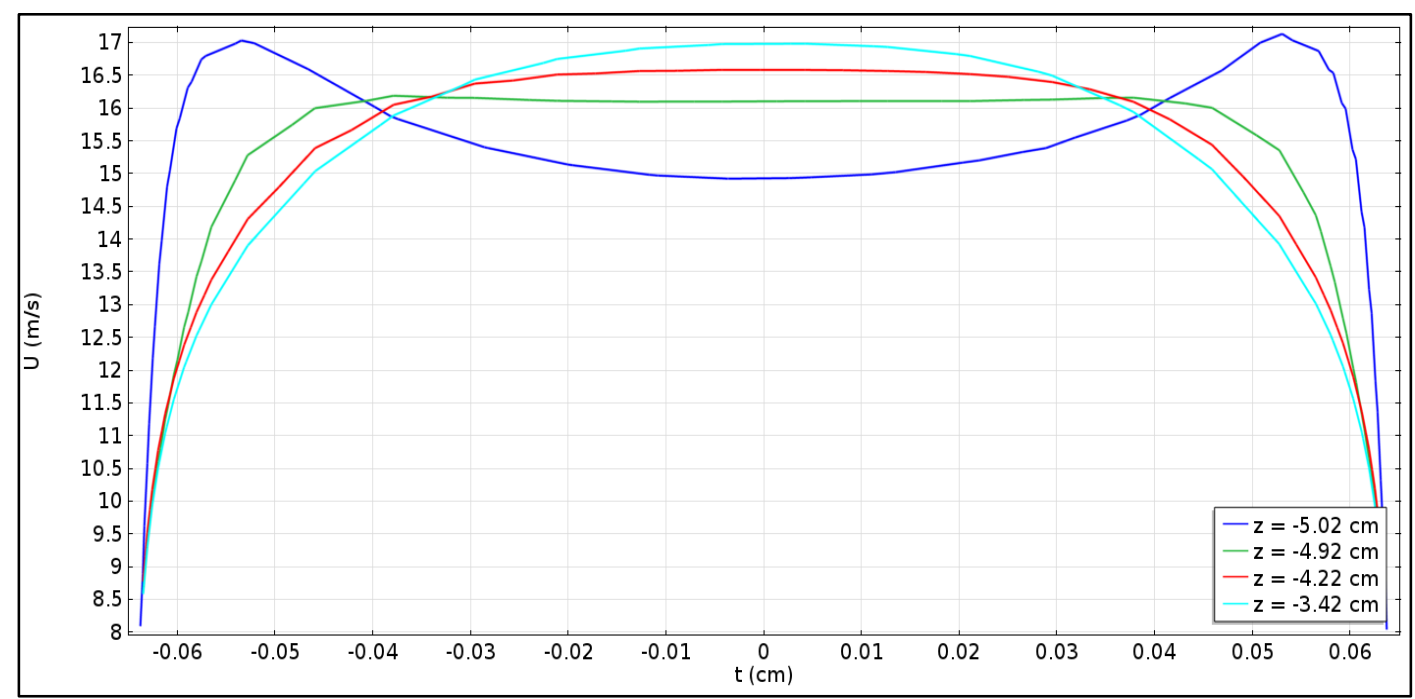

Fig. 7.14. Coupled Model Hydrodynamic Entrance Region Velocity Profiles.

Performing a surface integral of heat flux on the leading and trailing edge surfaces shows the total heat flow through each to be less than $1 \mathrm{~W}$. This is a completely trivial quantity with respect to the magnitude of the nuclear heat generation. The coupling mechanisms attached to these surfaces can therefore be removed without affecting any noticeable change in the model's solution. With the coupling boundaries dropped, both surfaces - in both domains - are treated as insulated boundaries. Although a trivial quantity compared to the millions of degrees of freedom in the model, this allows a few hundred unnecessary coupling variables to be removed from the model. The greatest gain is in the increased simplicity of the coupling mechanism which is now active only on axial faces. 


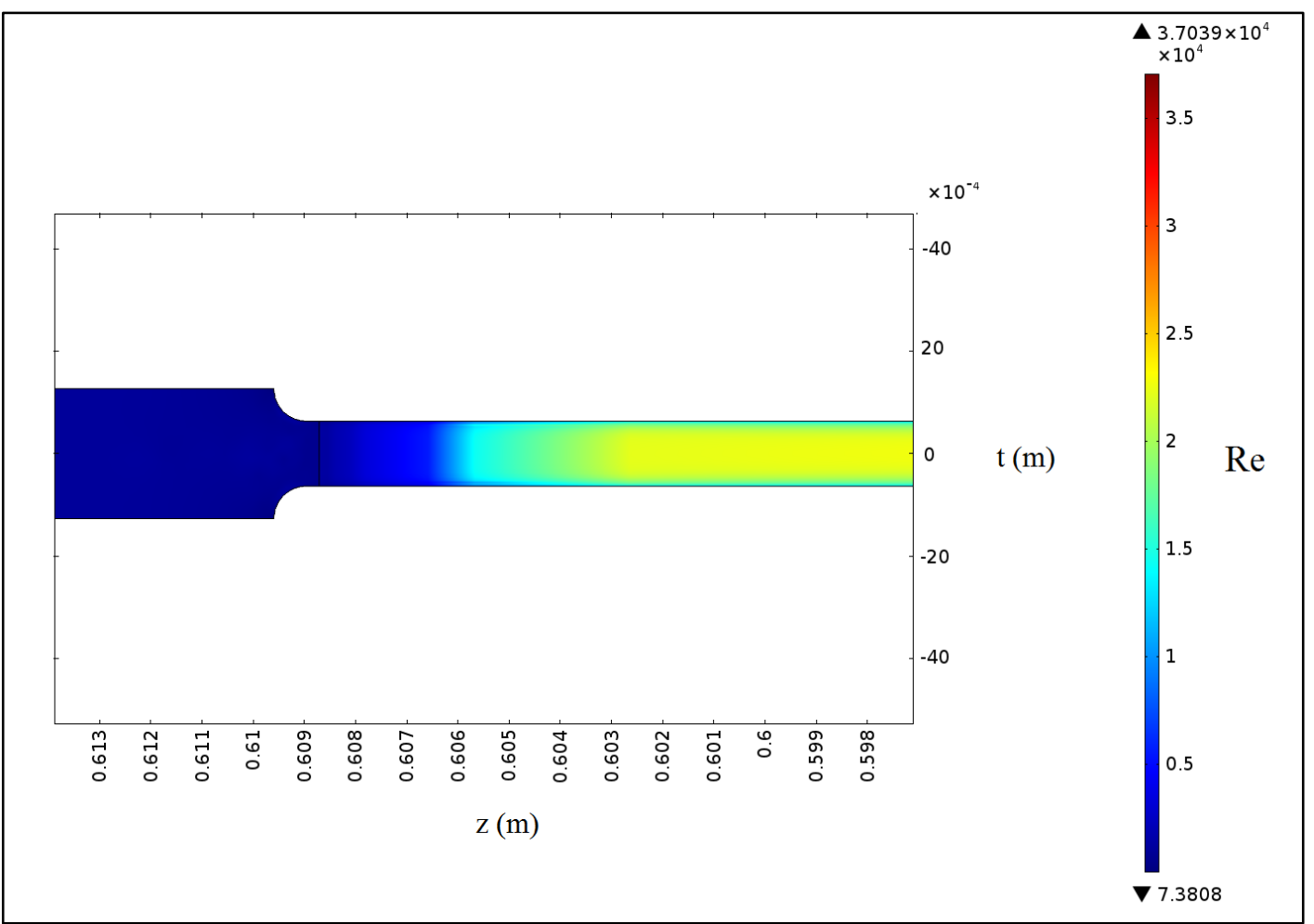

Fig. 7.15. Coupled Model Entrance Region Reynolds Number Profile.

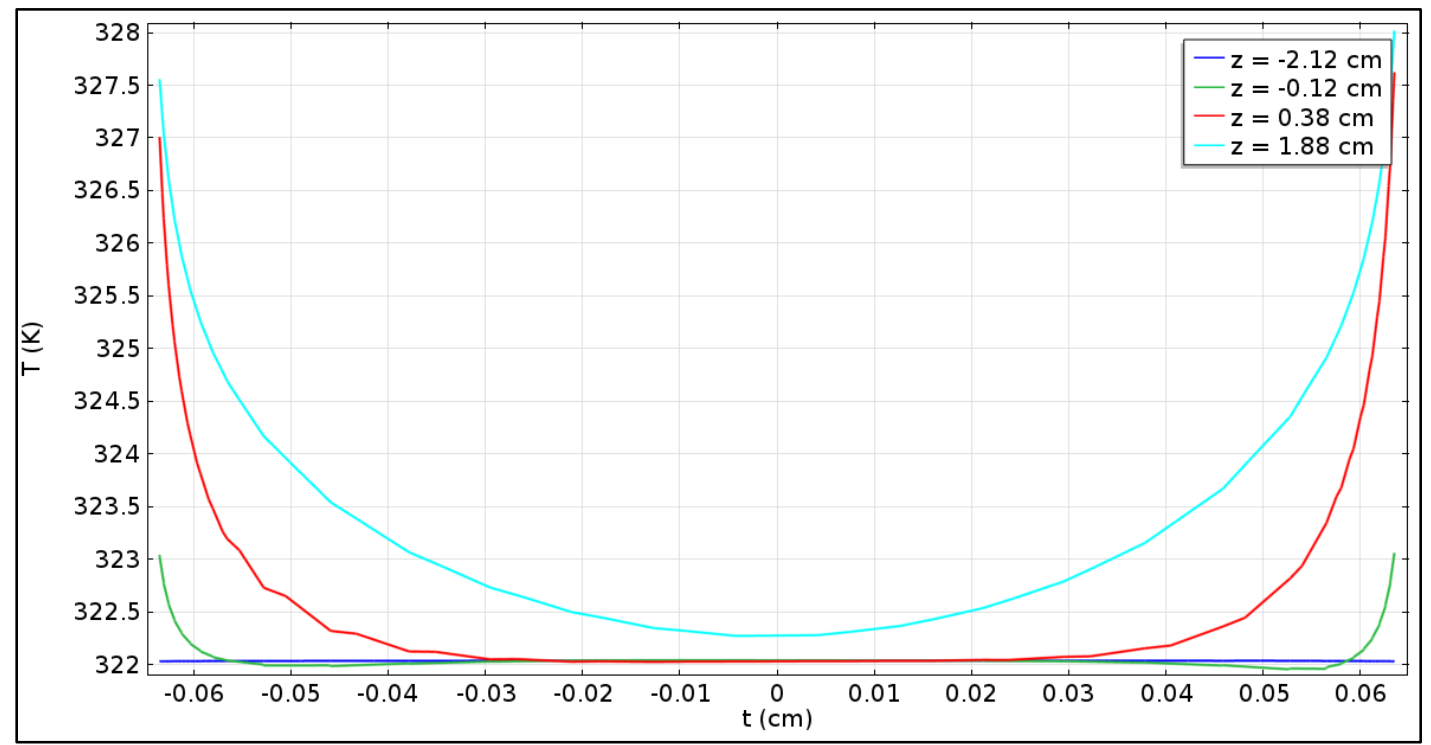

Fig. 7.16. Coupled Model Thermal Entrance Region Temperature Profiles. 


\section{E. Paneling}

The concept of paneling was first tested using a conceptual model. This model substituted the highaspect ratio fuel plate with a simple block with two opposing faces cut in half and coupled to $2 \mathrm{D}$ coolant channel slices. Heat flow was triggered in the system by a specified midplane temperature profile. The interactions of the conceptual model are shown in Fig. 7.17.

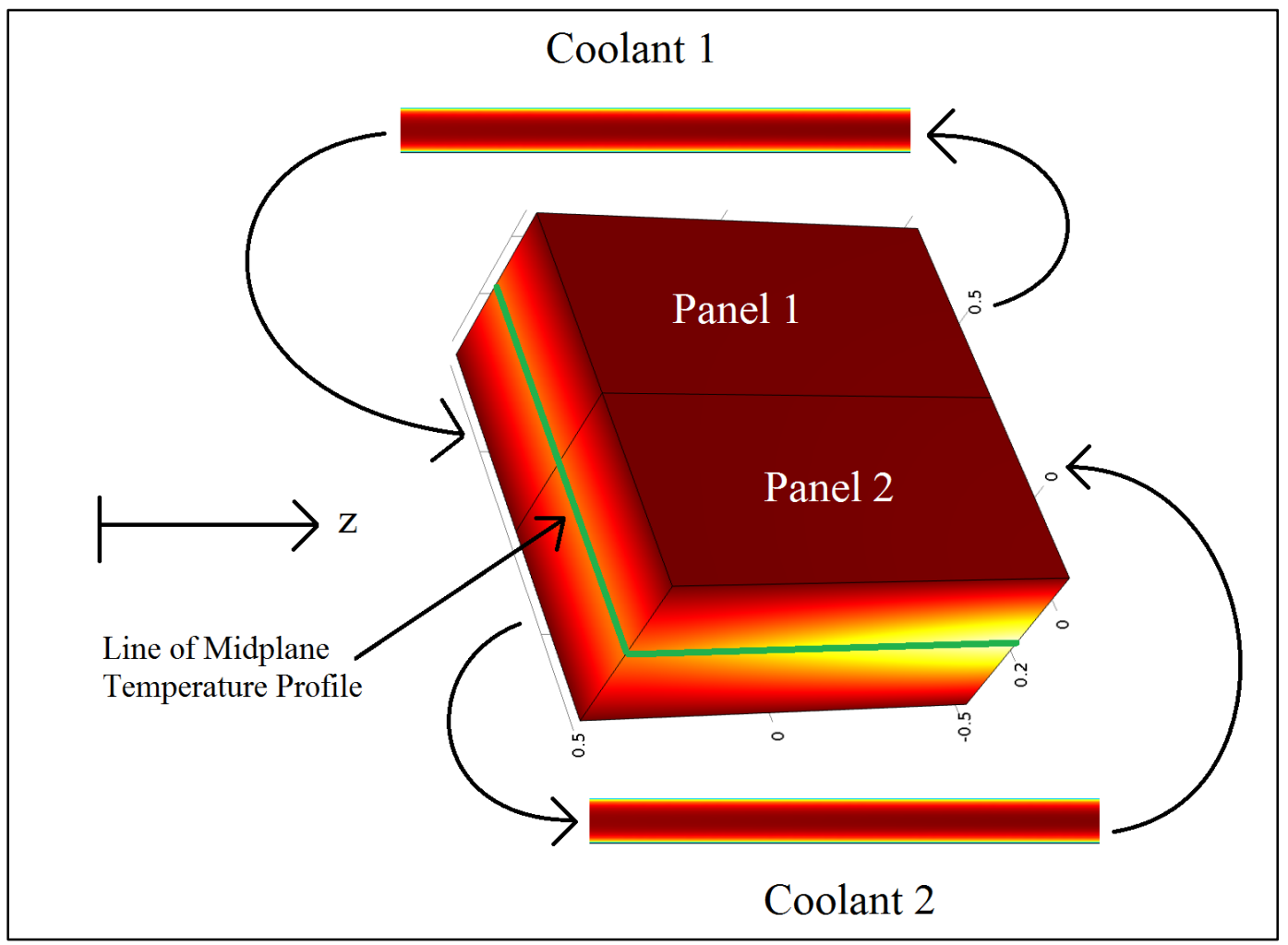

Fig. 7.17. Conceptual Paneling Model Diagram.

The model performed exceedingly well, providing validation for the paneling concept. The bifurcated bulk temperature profile that the model yielded, as projected onto the panels themselves, is shown in Fig. 7.18. The gain of the model was that instead of imposing a single bulk temperature across the span of the heated body, a two-part discontinuous temperature profile could be imposed. Despite the discontinuity in the bulk temperature profile the conceptual model produced a smooth surface temperature, as shown in Fig. 7.19. The imposed midplane temperature profile is included for reference. 


\section{Panel 1}

Panel 2

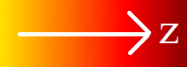

Fig. 7.18. Conceptual Paneling Model Bulk Temperature Projections.

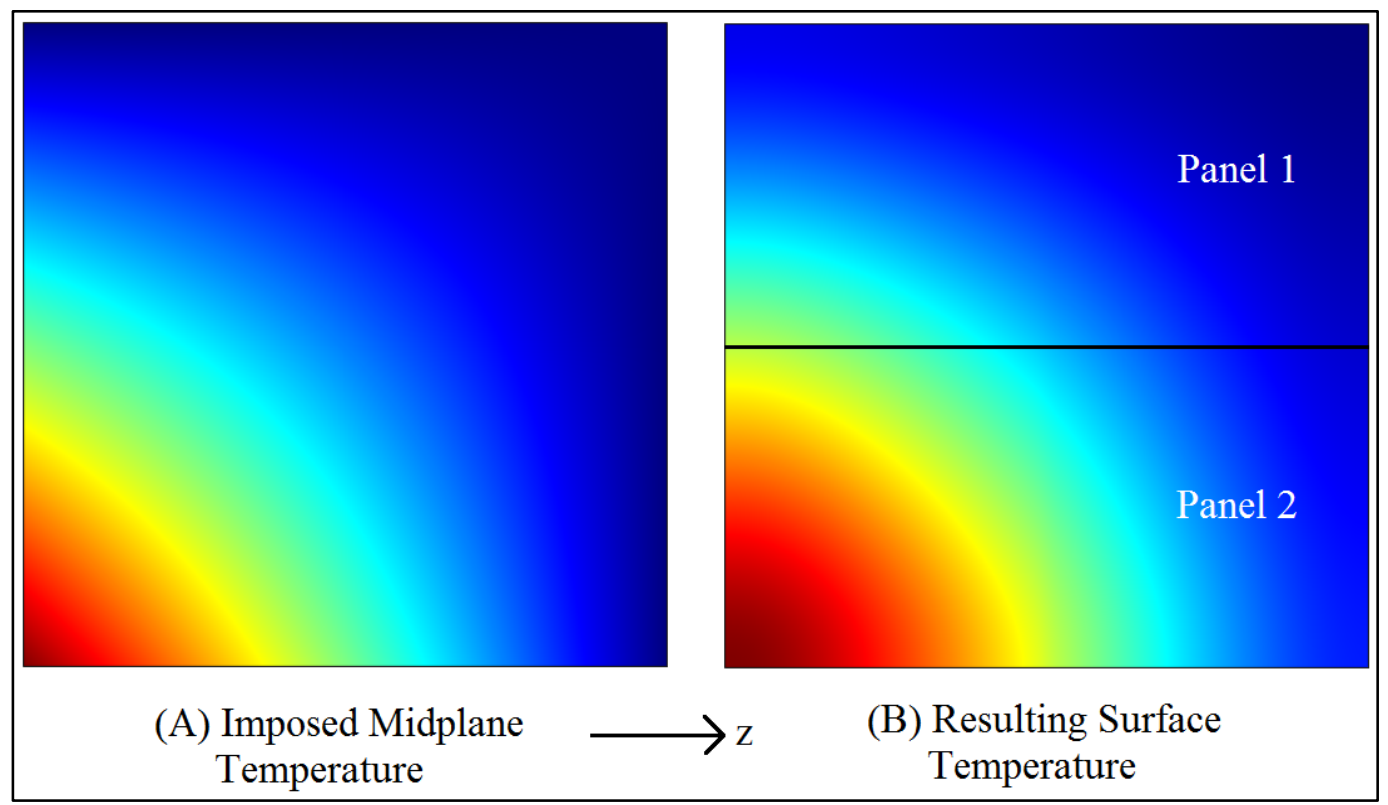

Fig. 7.19. Conceptual Paneling Model Surface and Midplane Temperature Profiles.

Temperature profiles are not on the same color scale. They are merely intended to provide a qualitative comparison.

The paneling concept failed to transition successfully to the coupled model. The issue arises from the high aspect ratio of the fuel plate. The coolant creates a path of least resistance, so to speak, for heat flowing out of the fuel meat. Given the extremely short distance between the two, compared to both the length and the span of the fuel plate, heat flow occurs almost exclusively in the $t$-direction, parallel to the 
normal of the fuel plate's surface. Any diffusion occurring within the fuel plate in the $s$ - or $z$-directions is miniscule in comparison. As a result, the surface temperature profile that this yields contains an artifact of the bifurcated bulk temperature profile. This surface temperature profile is shown in Fig. 7.20. There is a "kink" visible running down the axial midline of the temperature profile. It is likely that with an increasing number of panels the severity of the kinks between each would decrease. Eventually they would decrease to the point that they became trivial. The requisite number of panels would likely overencumber the model, however, and negate the benefit of its lowered computational expense. As such, the paneling concept was not pursued further, it being deemed incompatible with the fuel plate geometry.

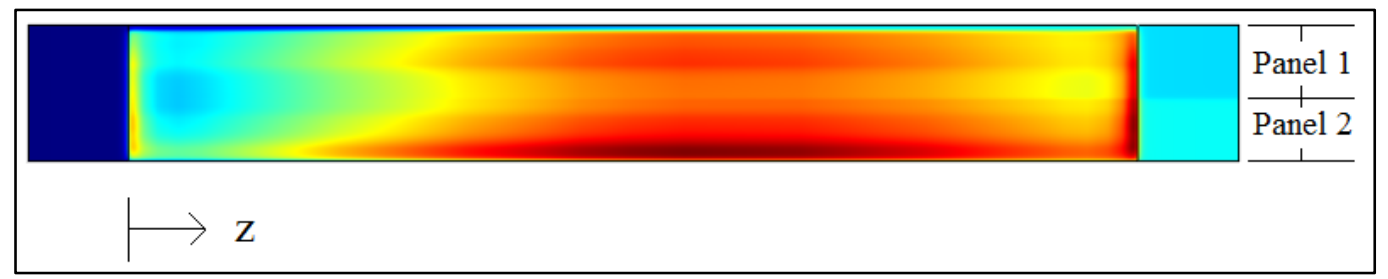

Fig. 7.20. Coupled Paneled Model Concave Fuel Plate Surface Temperature Profile.

\section{F. Model Comparisons}

Solution comparisons in the fuel plate are relatively straightforward given that the geometry itself is identical in both models. Coolant channel comparison are more difficult due to the dimensional disparity between the two models. While comparing profiles of surface variables provides the most information about the state of a given solution, it is less useful for quantifying differences between solutions. Consider, for example, Fig. 7.21 in which the temperature profile on the concave surface of the fuel meat in each model is shown. The surface profile comparison readily conveys the similarity in solution between the two models, showing similarly topographies and corresponding hot and cold patches. It does provide a clear understanding of the difference in magnitude between the solutions at any given point, however.

For this purpose, consider the 1D temperature data from Axial Lines 1 and 2 plotted in Fig. 7.22. Axial Lines 1 and 2 were placed such that they ran through sections of relatively high and low temperature, respectively. As was expected for the coupled model, in areas of higher temperature, Axial Line 1, the difference in observed temperatures between models is greatest. In areas of mid to lower temperature, Axial Line 2, the observed temperatures are in much closer agreement. This is a combination of the smoothing effect of the bulk coolant slice used in the coupled model which affects 
local minima and maxima as well as the sacrifice to accuracy made for the sake of computational cost associated with the final coupled model which lowers the temperature of the entire model by a few Kelvin.

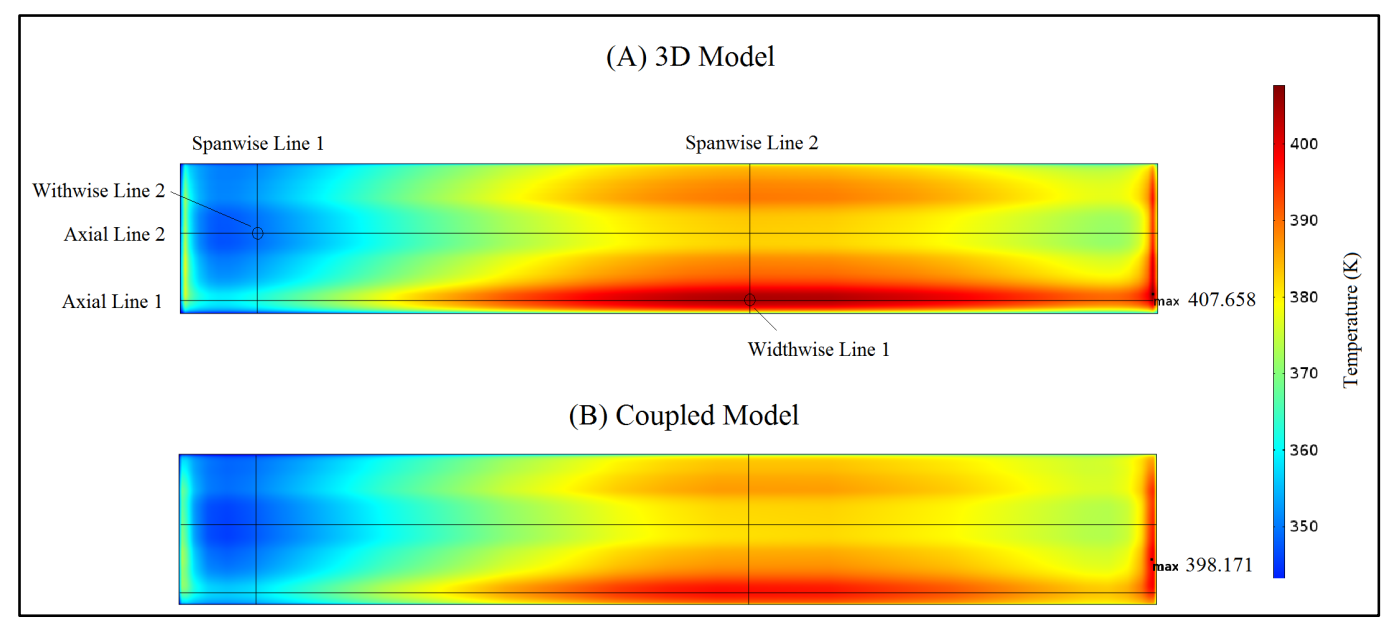

Fig. 7.21. Fuel Meat Concave Surface Temperature Profile Comparison.

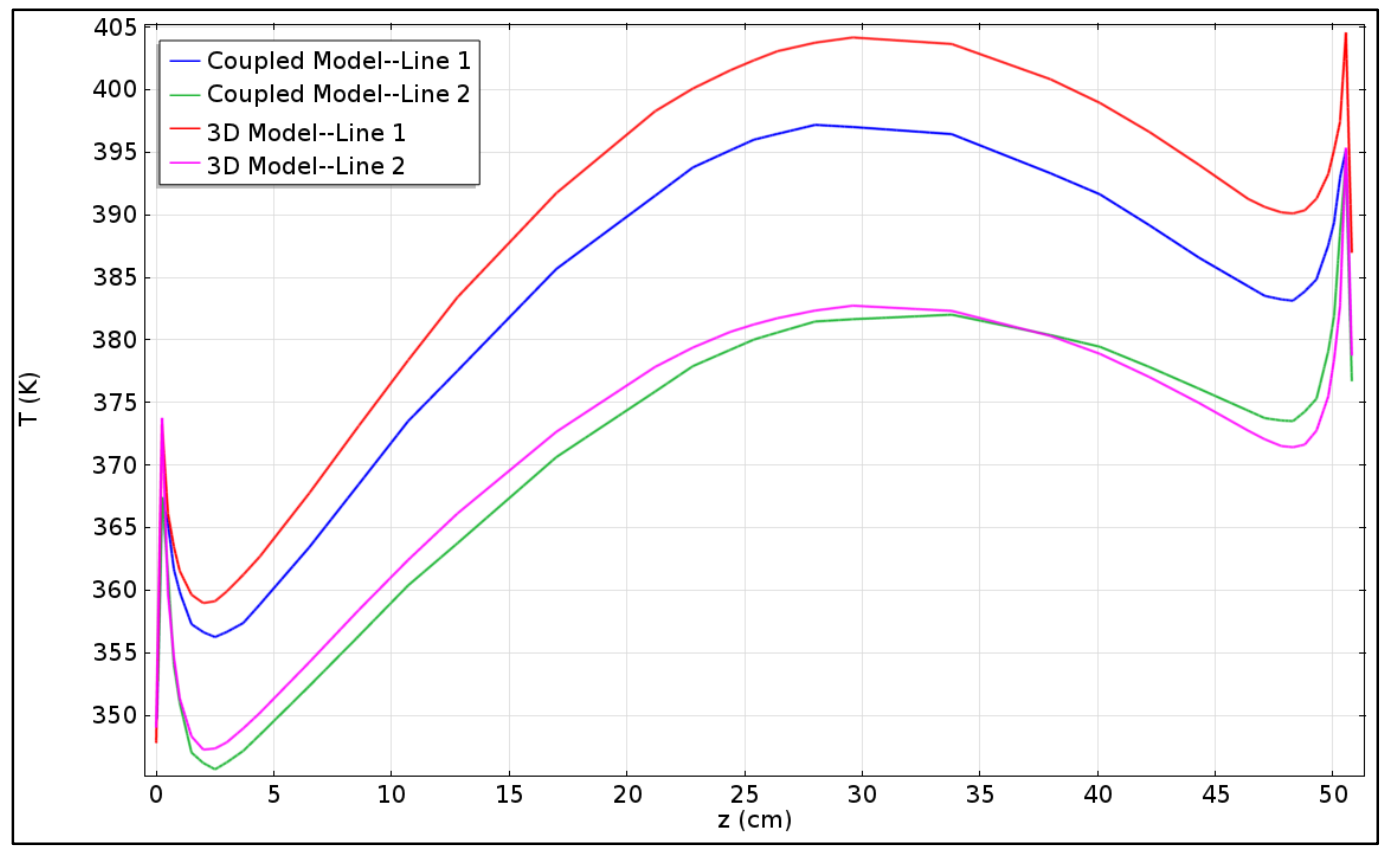

Fig. 7.22. Fuel Meat Concave Surface Axial 1D Temperature Profiles. 
The 1D temperature comparison does a much better job of quantifying the difference between the two solutions than the $2 \mathrm{D}$ comparison does. The trade off with the $1 \mathrm{D}$ comparison is that it conveys much less information than the surface comparison, the former having one fewer dimension than the latter. For this reason, model comparisons, where appropriate, will be presented in two parts: one part to provide a qualitative comparison between models and another to quantify the difference between them.

The same quantitative comparison can be made in the spanwise direction using temperature data from Spanwise Lines 1 and 2, as shown in Fig. 7.23. Again, the hotter of the two lines, Spanwise Line 2, shows the greatest temperature disparity between the two models. In Spanwise Line 2's temperature profiles alone the effect that higher temperature has on differences between the model is also apparent. It is at the profile's lowest point, ignoring its outer edges, in which the two profiles are in the closest agreement. Performing a temperature average along Spanwise Line 2 in the coupled model shows that the average temperature is $385.8 \mathrm{~K}$ which is very close to the region in which the profiles are in the closest agreement.

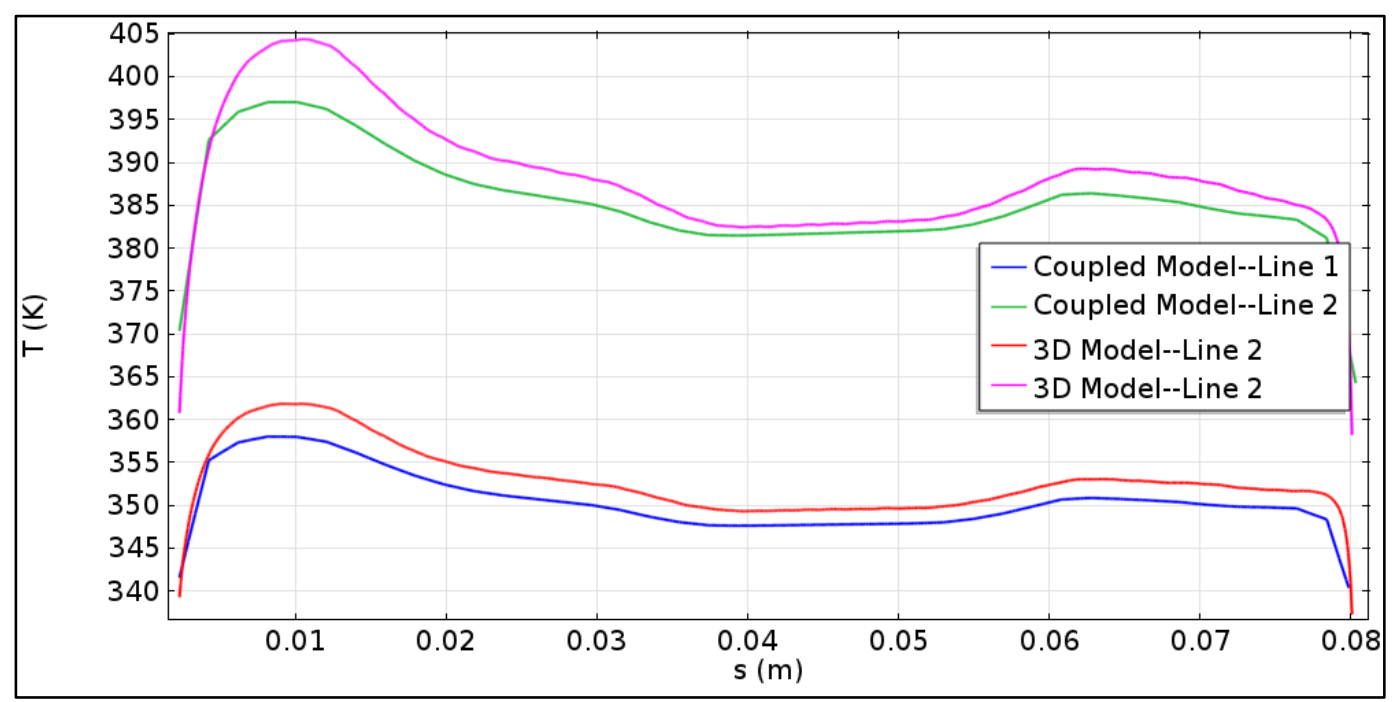

Fig. 7.23. Fuel Meat Concave Surface Spanwise 1D Temperature Profiles.

Looking at a cross-sectional plane cut through the entire model along Spanwise Line 2, a topographical comparison can be made in the $s-t$ plane as well. This comparison is shown in Fig. 7.24. Both models show the same general topography with the coupled model showing slightly higher temperatures along the inner side plate border at top left than the 3D model. This is likely due to the fact that local fuel plate temperatures fall below the spanwise average in this area and the bulk coolant is thus 
higher than it should be. A small part of it could also be the removal of the side plate. A more direct comparison can be made between the models by looking at the 1D temperature profiles along Widthwise Line 1 drawn on both plots. Temperature data is plotted along Widthwise Line 1 in Fig. 7.25. The widthwise line corresponds roughly to the intersection of Axial Line 1 and Spanwise Line 2 in Fig. 7.21 and serves as a high temperature comparison. A low temperature comparison is made with Widthwise Line 2 near the intersection of Axial Line 2 and Spanwise Line 1, as shown in Fig. 7.26. As with the 1D axial and spanwise temperature comparisons, the higher the local temperature the greater the temperature disparity between the two models. In the cold widthwise comparison the coolant temperatures are nearly aligned.

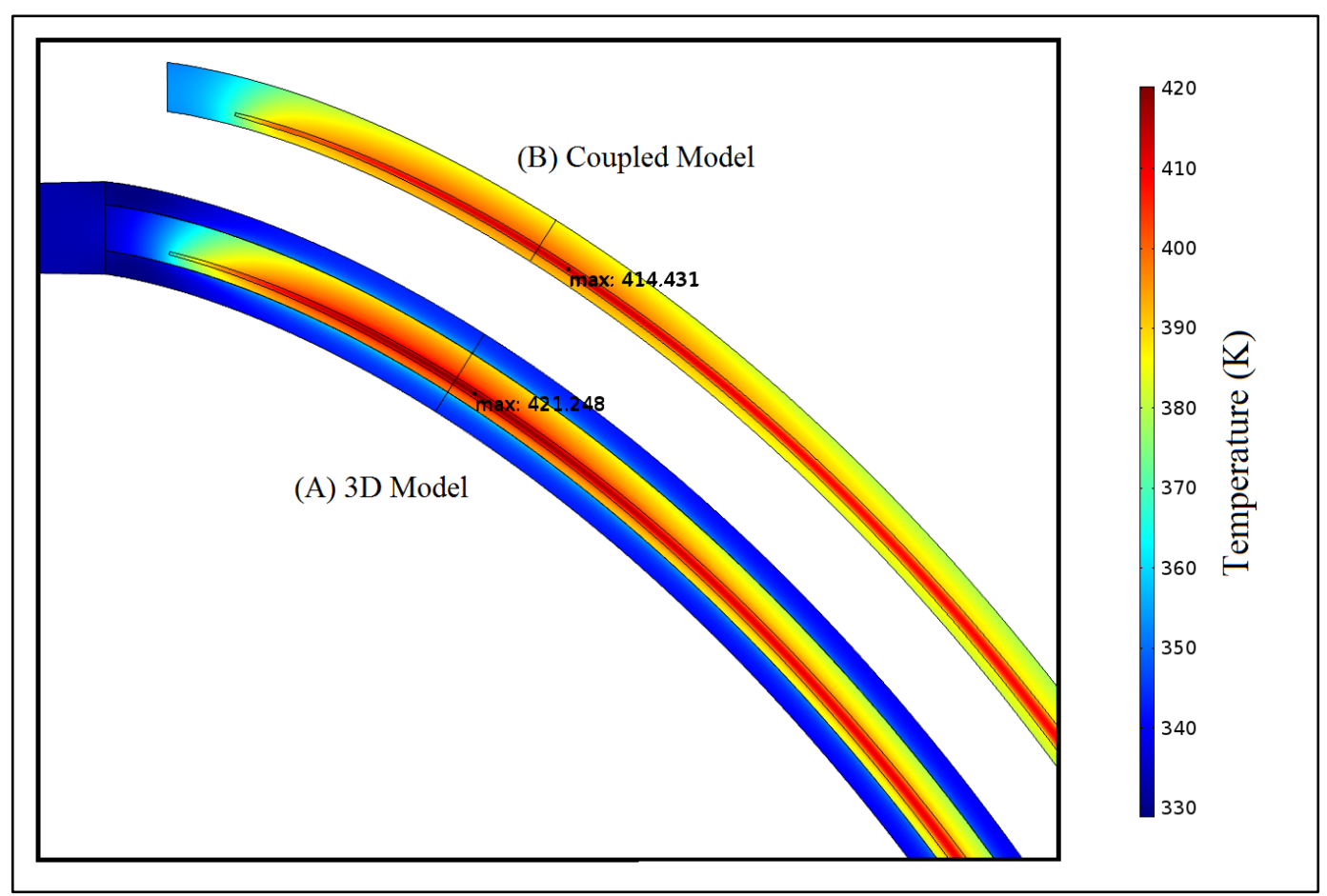

Fig. 7.24. Cross-Sectional Cut-Out Temperature Profile Comparison. The 3D model crosssection is shown with all geometry elements present including the fuel meat, fuel plate, coolant channels, and inner side plate. Given the dimensional disparity across the coupling interface in the coupled model, only the fuel meat and fuel plate are shown. 


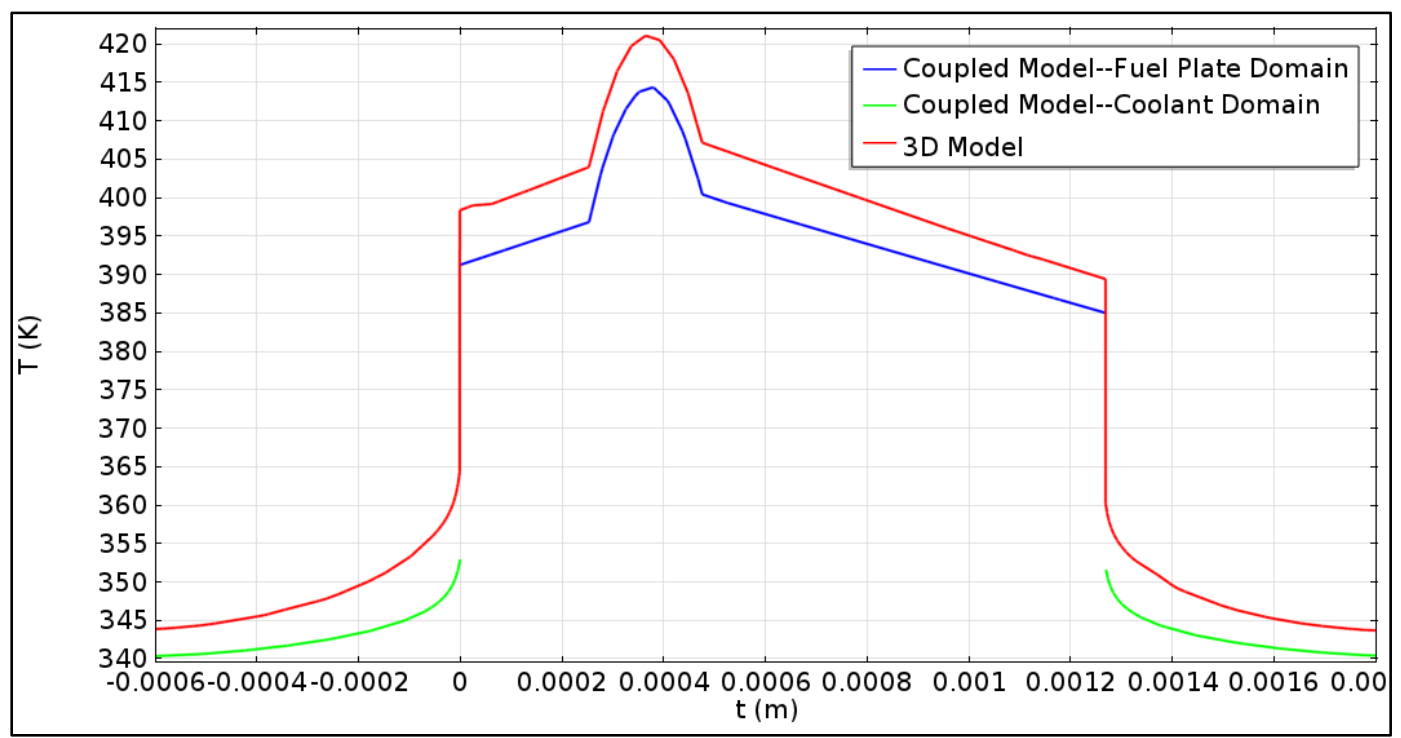

Fig. 7.25. Cross-Sectional Cut-Out Widthwise Line 1 1D Temperature Profiles. While the 3D profile is plotted as a single entity, the coupling interface in the coupled model necessitates that the fuel plate and coolant temperature profiles be plotted separately.

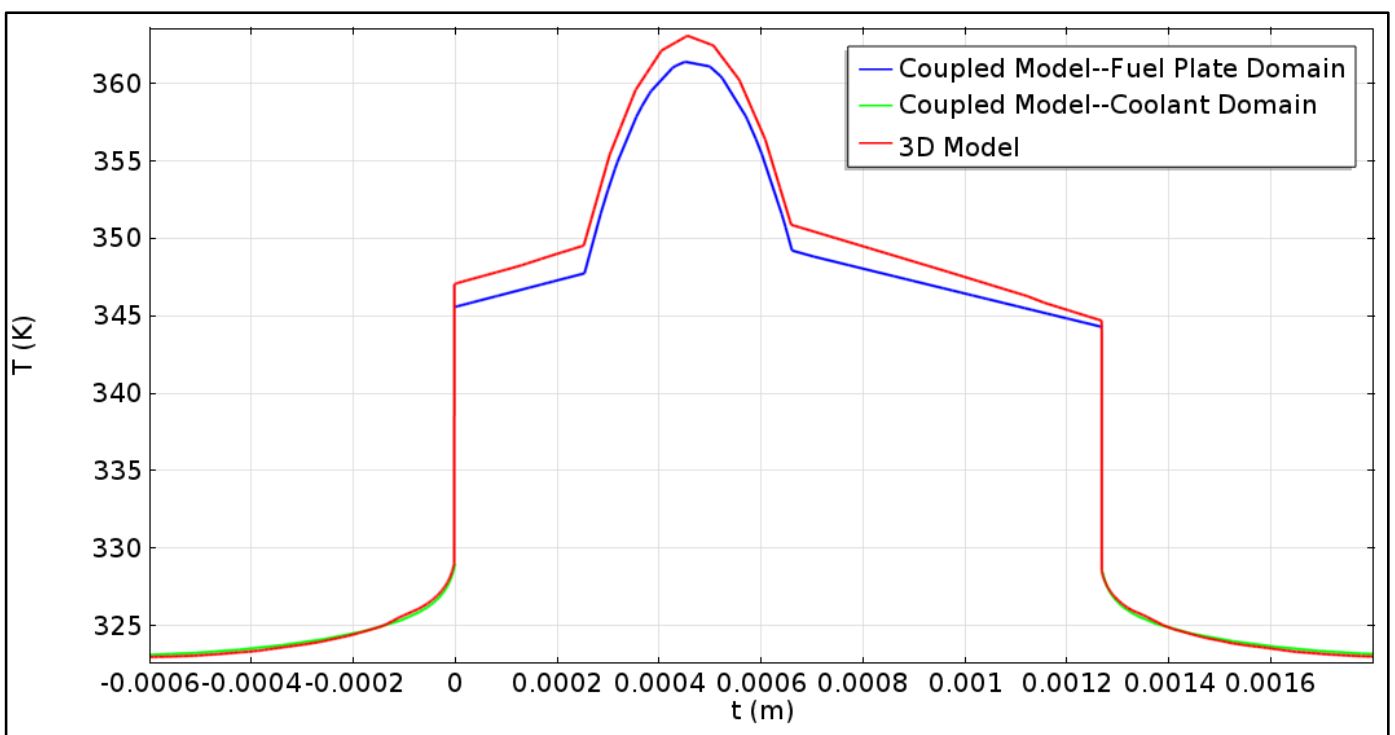

Fig. 7.26. Cross-Sectional Cut-Out Widthwise Line 2 1D Temperature Profiles. While the $3 \mathrm{D}$ profile is plotted as a single entity, the coupling interface in the coupled model necessitates that the fuel plate and coolant temperature profiles be plotted separately.

Qualitative comparisons can be made between the coolant channels using a $t$-z plane oriented along the same widthwise lines in Fig. 7.24. Given the extremely high aspect ratio of the coolant channel in the $t$ direction, two magnified velocity contours are presented which focus on the leading and trailing edge in 
Figs. 7.27 and 7.28, respectively. Both models' leading edge velocity contours look very similar from the effects of the mid-plate stagnation point to the rapid of flow acceleration leading from the rounded leading edge to the constant-width fuel plate. The effects of the boundary layer are more pronounced in the 3D model's solution.

The topography at the trailing edge is dominated by "cores" of high- and low-velocity coolant that persist downstream of the coolant channel and the fuel plate, respectively. Both high- and low-velocity cores appear to persist farther downstream in the coupled model than in the $3 \mathrm{D}$ model. This is to be expected as the coupled cores can only diffuse in the $t$-direction while the $3 \mathrm{D}$ cores experience a lesser diffusion in the $s$-direction as well. When considering these differences, however, it is important to note that the 3D model velocity contour is a local dissection of an explicit coolant channel while the coupled model profile is a homogenization of the entire span of the channel. The difference in exit core lengths could simply be attributed to a variation in upstream fuel plate temperatures.

Both models capture the slight misalignment in coolant channel exit core velocity caused by the nonsymmetric placement of the fuel meat inside the fuel plate. This yields a hot side (the concave side) and a cold side (the convex side). In the velocity profiles the hot side is on the right-hand side of the coolant channel. More energy enters the coolant channel from this, creating a slight non-uniformity in pressure across the width of the channel. At the trailing edge, this non-uniform pressure, higher on the right-hand side, pushes the flow slightly to the left. This non-symmetric pressure profile is shown in Fig. 7.29. In both models, the velocity field reaches a uniform profile well before the outlet, located roughly another $2 \mathrm{~cm}$ below the bottom cut-off of the figures, indicating that the exit region is sufficiently long enough to justify the use of a uniform pressure boundary condition. 


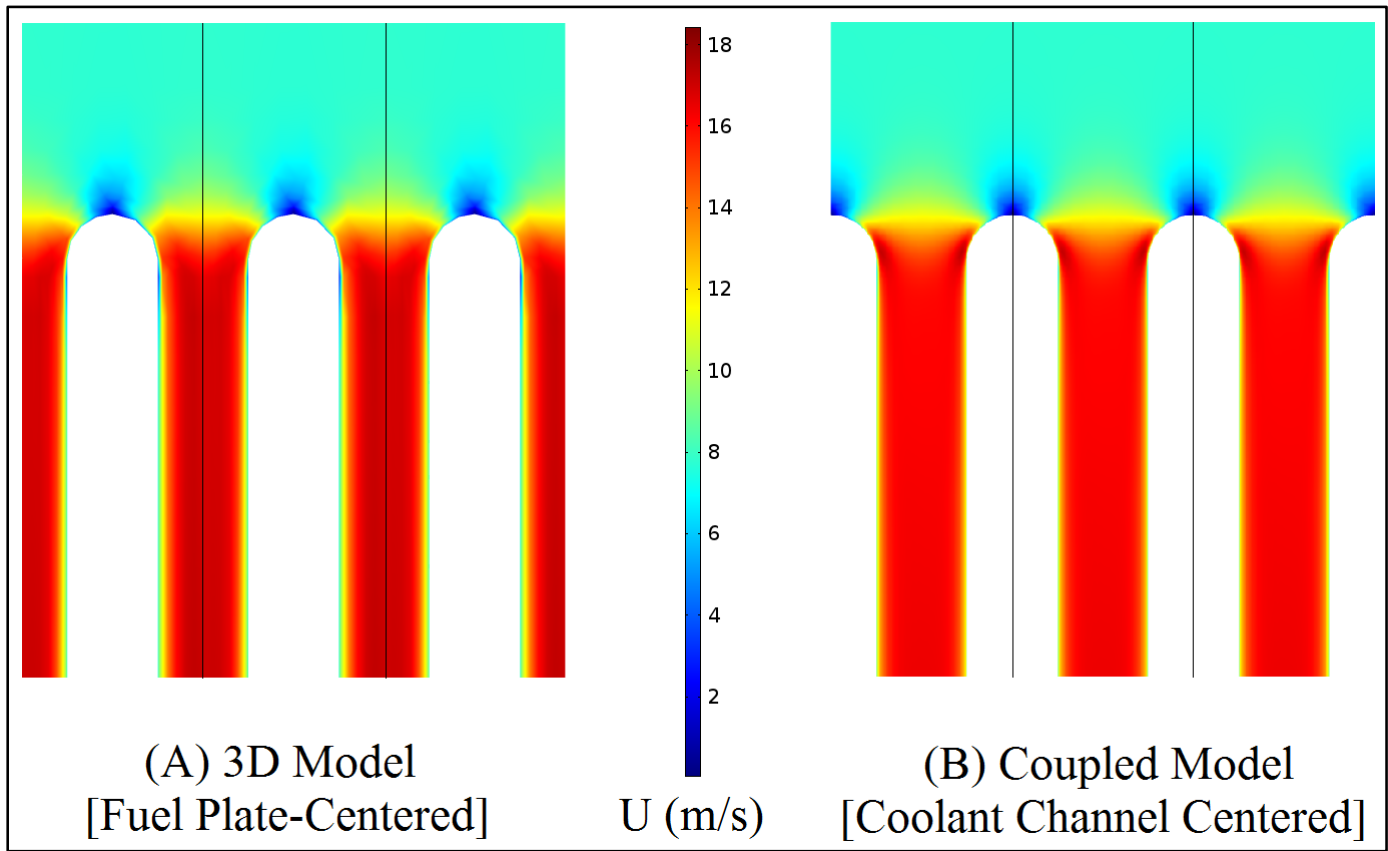

Fig. 7.27. Coolant Channel Leading Edge Total Velocity Contour Comparison. Note that the coolant domain in each model consists of only a single channel. Three channels are presented side-by-side in order to better show the profile continuity across the periodic boundaries.

The smoothing effect of the coolant channel slice in the coupled model decreases with increasing distance from the coolant-fuel plate boundary. This diminishing effect with increasing distance can be illustrated by comparing maximum temperature values at varying distance from the coolant-fuel plate boundary. The effect's propagation is shown in Table. 7.6. The ad hoc temperature error, $e_{T}$, from the coupled model fuel plate mesh convergence study can be reapplied here with the 3D model's temperature at each point serving as the reference value. Not only does the difference in maximum temperature decrease as the points move inward but the percent error decreases as well. The coolant slice's smoothing effect has very nearly worn off by the point it has propagated into the interior of the fuel meat. 


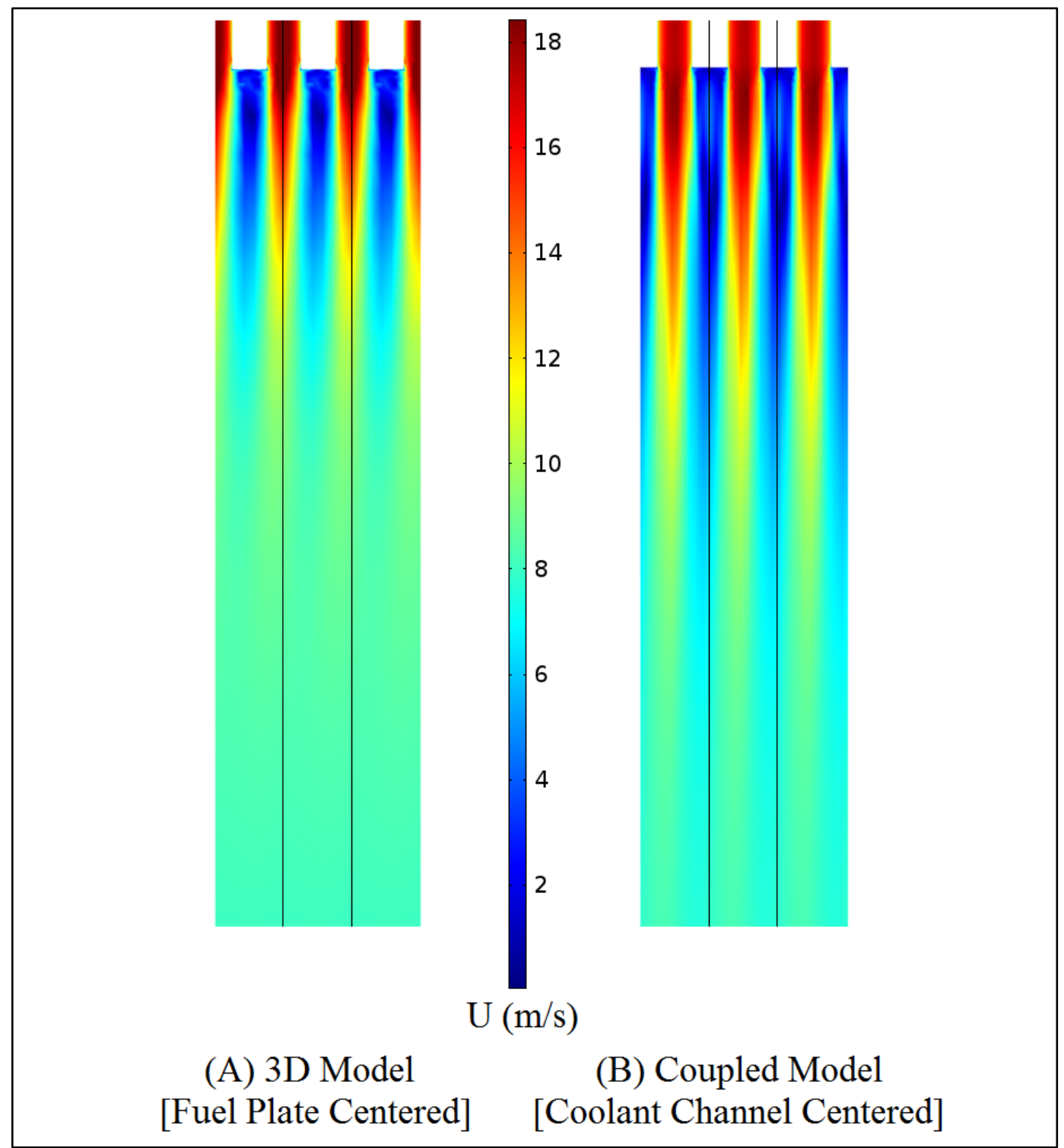

Fig. 7.28. Coolant Channel Trailing Edge Total Velocity Contour Comparison. Note that the coolant domain in each model consists of only a single channel. Three channels are presented side-by-side in order to better show the profile continuity across the periodic boundaries. 


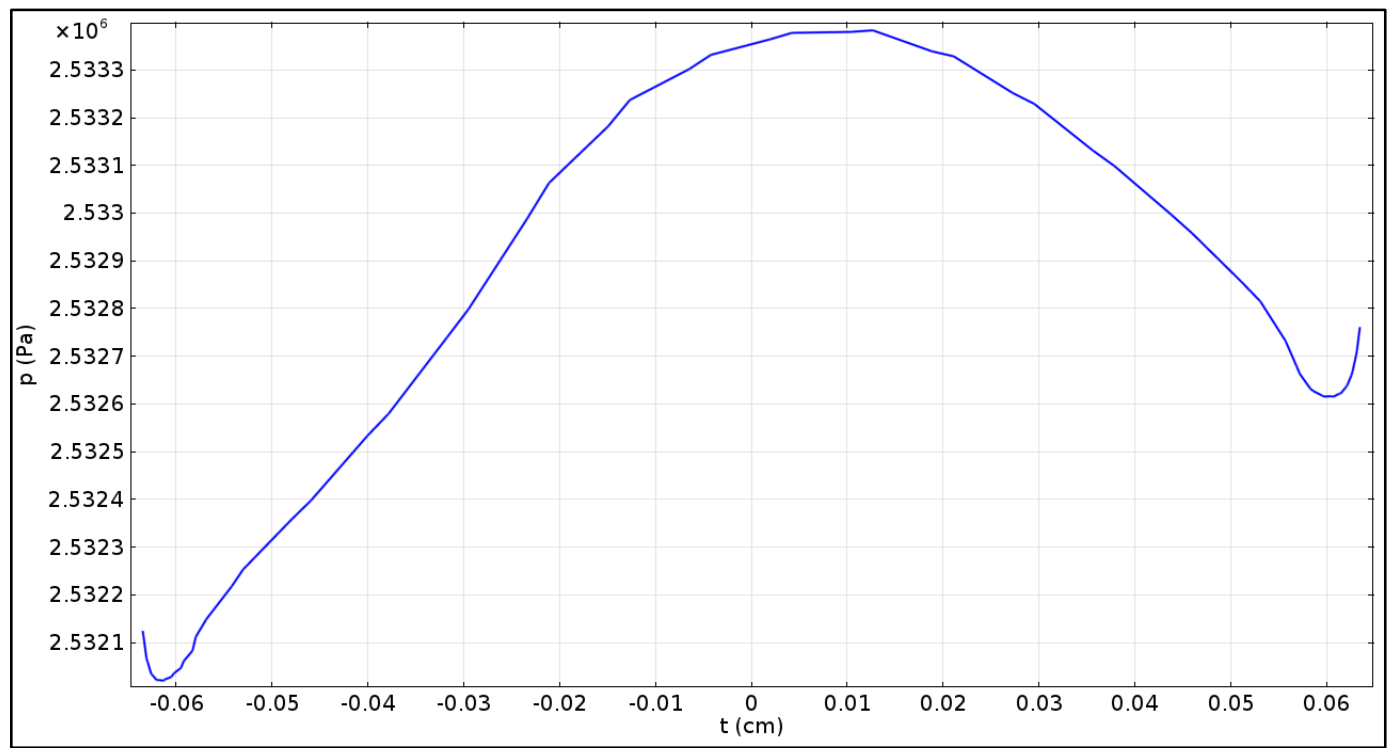

Fig. 7.29. Coupled Model Non-symmetric Trailing Edge Pressure Profile. The trailing edge pressure profile was taken from a widthwise line located at $z=55.78 \mathrm{~cm}, 0.1 \mathrm{~cm}$ above the trailing edge itself.

Table 7.6: Propagation of Coolant Smoothing Effect into Fuel Plate

\begin{tabular}{|c|c|c|c|c|}
\hline \multirow{2}{*}{ Domain } & \multicolumn{2}{|c|}{ Max Domain T (K) } & \multirow{2}{*}{$\Delta \mathrm{T}(\mathrm{K})$} & \multirow{2}{*}{$e_{T}(\%)$} \\
\cline { 2 - 3 } & 3D & Coupled & & \\
\hline Coolant & 375.3 & 364.6 & 10.7 & 10.5 \\
\hline Fuel Plate Surface & 403.0 & 393.7 & 9.3 & 7.2 \\
\hline Fuel Meat Surface & 409.1 & 400.7 & 8.4 & 6.2 \\
\hline Fuel Meat Internal & 431.8 & 429.6 & 2.2 & 1.4 \\
\hline
\end{tabular}

\section{G. Computational Efficiency}

It is indisputable that the coupled model trades a small measure of accuracy for the ability to replicate core conditions much faster and with fewer computational resources. While the former sacrifice is small, the latter gain is considerable. The solution times for both models are given in Table 7.7 along with the computational resources required to obtain the solution. All runs were performed on the computer cluster Betty at ORNL. Eight of the nodes on Betty are reserved for COMSOL use. Each node possesses two AMD Opteron 6128 processors, 16 cores, and 128 GB of RAM. For a small trade in accuracy, the coupled model can be run in $\sim 1 / 23^{\text {rd }}$ the time of the $3 \mathrm{D}$ model using $1 / 3^{\text {rd }}$ of the computational resources. The true decrease in required computational resources is even greater. A single run of the coupled model requires less than half of the available resources on a single Betty node. 


\begin{tabular}{|c|c|c|c|c|}
\hline \multicolumn{5}{|c|}{ Table 7.7: Coupled vs. 3D Speed-Up } \\
\hline Model & $\begin{array}{c}\text { Computation } \\
\text { Time (hrs) }\end{array}$ & $\begin{array}{c}\text { Mesh Elements } \\
\text { (in thousands) }\end{array}$ & $\begin{array}{c}\text { Degrees of Freedom } \\
\text { (in thousands) }\end{array}$ & $\begin{array}{c}\text { Betty } \\
\text { Nodes }\end{array}$ \\
\hline 3D & 69 & 2311 & 6465 & 3 \\
\hline Coupled & 3 & 21 & 288 & 1 \\
\hline
\end{tabular}

\section{H. Justification of Selected Residual Tolerance}

All solutions presented in this section were performed using a relative residual of $1.00 \times 10^{-2}$ which was deemed sufficient to ensure adequate convergence. To demonstrate this point, the final coupled model was run using a relative residual of $1.00 \times 10^{-5}$. A comparison of the two solutions is shown in Table 7.8. Any change in solution in the fuel plate fails to register with five significant digits. A slight drop in the change in coolant enthalpy is measured by the higher tolerance case but its effect is so minimal that it doesn't register a change in the maximum temperature to four significant digits. The only truly nontrivial effect that increasing the tolerance had was to increase the solution time substantially.

\begin{tabular}{|c|c|c|c|c|c|c|}
\hline \multicolumn{7}{|c|}{ Table 7.8: Relative Tolerance } \\
\hline Tolerance & $\begin{array}{c}\text { Solution } \\
\text { Time (hrs) }\end{array}$ & $E_{p, \text { out }}^{\prime}$ & $\Delta H^{\prime}{ }_{c}$ & $e_{c}(\%)$ & $e_{g}(\%)$ & $\begin{array}{c}\text { Max Fuel } \\
\text { Meat T [K] }\end{array}$ \\
\hline $1.00 \times 10^{-2}$ & 3 & 1.0002 & 1.0358 & 3.557 & 3.577 & 429.6 \\
\hline $1.00 \times 10^{-5}$ & 27 & 1.0002 & 1.0304 & 3.011 & 3.035 & 429.6 \\
\hline
\end{tabular}




\section{Conclusion}

Without experimentally verified data for LEU core operating conditions against which to compare the coupled model, it is impossible to say definitively that it is a sufficiently accurate tool with which to model HFIR core thermal-hydraulics. The close agreement between the coupled model and the more complete 3D model, however, is a strong indicator that both provide good approximations of core conditions. In this regard, the coupled model has proven itself to be an exceedingly more efficient and less time-consuming alternative to the $3 \mathrm{D}$ model capable of producing results of a comparable quality.

With the coupled model's improvements over the 3D model CFD analysis of HFIR core thermalhydraulics become a realistic alternative to existing modeling methods. Currently, core thermalhydraulics are modeled using the systems-level code RELAP5 and SSHTC. Both are heavily dependent upon experimentally-obtained correlations and thus incapable of accurately modeling, and predicting, unobserved core operating conditions. This limitation is true for both non-ideal operating conditions as well as LEU fuel operating conditions.

Of the two existing codes, SSHTC is more closely related to the coupled model with regards to its purpose. Both models are concerned primarily with fuel plate temperatures, both are driven by the MCNP nuclear heat generation profile, and both utilize the modified Hausen correlation to communicate with a simplified coolant domain via a convective boundary. The coupled model, as demonstrated, simplifies the coolant by reducing it to a two-dimensional slice. SSHTC, conversely, removes the coolant entirely, instead relying on fixed correlations to calculate bulk fluid properties as a function of fuel plate surface temperatures. The problem with this approach, not including its heavy dependence upon experimental observations, is the fact that coolant properties, calculated as functions of boundary temperatures, are then used in convective boundary conditions to regulate those same boundary temperatures. The ability to iteratively communicate between the two domains in such a manner requires prior experimental knowledge of operating conditions. The method is thus only useful for returning fuel plate temperatures for an already observed operating state. It does not retain confidence as a predictive modeling tool

The fuel plate in SSHTC is modeled as a series of isolated one-dimensional cells oriented in the $t$ direction perpendicular to the fuel plate surface. Each cell communicates with the surface convective boundary adjacent to it but not with any of the cells beside it, essentially neglecting fuel plate conduction in the $s$ - and $z$-directions. The coupled model accounts for fuel plate conduction in all directions. The simplified coupled coolant domain is also, importantly, governed by the physics of turbulent pipe flow via the RANS N-S equations, not experimental correlations. This ensures that, unlike SSHTC, the coupled 
model can confidently adapt to changes in operating conditions without losing validity. As the coupled model currently stands, in its single plate-single channel configuration, it is already a superior replacement to the existing SSHTC model currently in use.

Additionally, this thesis was begun with the intent to create a simplified model that could eventually be expanded to model the entire core. It is doubtful that the coupled model is such a model. It does, however, greatly expand the computational range possible for a single model. The 3D model, with its gratuitous DOF, would be hard-pressed to encapsulate even two adjacent fuel plates and their surrounding coolant channels in a single model. It is most likely possible with the available resources on Betty, but only just. The coupled mode, by contrast, could be expanded to encapsulate 22 fuel plates and coolant channels for the same DOF as the 3D model, assuming a linear expansion of DOF with added domains. If the doubling of the 3D model is assumed plausible then so would be the creation of a 44 plate coupled expansion. Additional improvements need to be made to the model before all 540 fuel plates in the reactor core, both inner fuel elements and outer fuel elements, can be modeled together. Forty-four plates constitutes over a quarter of the 171 inner fuel elements, however, and would thus be sufficient to provide quarter-turn axial symmetry to a simulation of non-uniform inner element core operation.

There are additional improvements that could be made to the coupled model to further increase its efficiency. Chiefly, more advanced meshing techniques, some of them unavailable at the onset of thesisrelated work, would permit an even greater decrease in the DOF for the coupled model. Mapped meshes could be inserted into the entrance and exit coolant channel regions above and below the fuel plate region, respectively, to replace the computationally inefficient free-triangular mesh that exists there now. Virtual mesh elements could be used to replace the free-triangular $s-t$ face mesh extruded down the length of the fueled section of the fuel plate with a neater, more evenly-spaced mapped mesh. CAD repairing features could be used to remove the free-tetrahedral mesh in the rounded leading edge of the fuel plate with a similarly neat mapped mesh extruded along the span of the leading edge.

Another important advancement is finding the means to further separate the current segregated solution variable groups. As was mentioned in Section IV, the coupled model currently requires that temperature in both the fuel plate domain and the coolant domain be solved in the same segregated step. Otherwise the model fails. The means to break this limitation would permit the solution process for a larger domain to be split into three segregated steps solving for: solid temperature, coolant flow variables, and coolant turbulence variables. The addition of the solid temperature segregated step might appear trivial at first. But when one considers the size of the solid domain versus the coolant domain, and 
the use quadratic basis functions within, the DOF associated with the solid temperature variable are comparable to the DOF associated with solving $T, u$, and $p$ in the coolant.

The most important advancement to be made, however, is the ability to utilize domain decomposition to break an expanded model down into manageable chunks. Such a decomposition would allow a small subset of plates and coolant channels to be solved independently of the broader solution domain. After a single iterate is performed the domain subset would pass its boundary values to the adjacent domain subsets which would then perform a local iterate and pass their resultant boundary values forward. Theoretically, domain decomposition would permit the entire core to be modeled rather easily using the coupled model given the fact that the minimal computational resources of a given domain subset would no longer be a limiting factor in the model's size. The only remaining concern would be computation time, which could become significant. Still, this is more of an inconvenience than a true limitation.

The coupled model might not be the definitive answer to the question first posed in this thesis but it is a confident step in the right direction. 
List of References 
1 "History of HFIR," URL: http://neutrons.ornl.gov/facilities/HFIR/history/ [cited July $24^{\text {th }}, 2013$ ].

${ }^{2}$ Knight, R. W., and Morin, R. A. Fabrication Procedures for Manufacturing High Flux Isotope Reactor Fuel Elements-2. ORNL/6852. Oak Ridge National Lab, Oak Ridge, TN. 1999.

${ }^{3}$ Martin, R. C., Knauer, J. B., and Balo, P. A. "Production, Distribution and Applications of Californium-252 Neutron Sources." Applied Radiation and Isotopes, Vol. 53, No. 4, 2000. Pp. 785-792.

4 “The High Flux Isotope Reactor at ORNL," URL: http://neutrons.ornl.gov/facilities/HFIR/ [cited July $24^{\text {th }}$, 2013].

${ }^{5}$ Munger, F. "Weird and Wonderful: ORNL's High Flux Isotope Reactor Sometimes Attracts the Unusual," URL: http://blogs.knoxnews.com/munger/2012/06/weird-wonderful-ornls-high-flu.html [cited July 24th, 2013].

${ }^{6}$ Cheverton, R. D., et al. "An Embrittlement Rate Effect Deduced from HFIR That May Impact LWR Vessel Support Life Expectancy.” Nuclear Engineering and Design, Vol. 117, No. 3, 1989. Pp. 349-355.

${ }^{7}$ Ilas, G., and Primm III, R. Low Enriched Uranium Fuel Design with Two-Dimensional Grading for the High Flux Isotope Reactor. ORNL/TM-2010/318. Oak Ridge National Laboratory, Oak Ridge, TN. 2011.

8 "Reactor Core Assembly," URL: http://neutrons.ornl.gov/facilities/HFIR/reactorassembly.shtml [cited July $\left.24^{\text {th }}, 2013\right]$.

${ }^{9}$ Bowden, G. A., and Knight, R. W. Specifications for High Flux Isotope Reactor Fuel Elements HFIR-FE-3. ORNL/TM-9220. Oak Ridge National Laboratory, Oak Ridge, TN. 1984.

${ }^{10}$ Mo, S. C., and Matos, J. E. "A Neutronic Feasibility Study for LEU Conversion of the High Flux Isotope Reactor (HFIR)." 1997 International Meeting on Reduced Enrichment for Research \& Test Reactors, Argonne National Laboratory, Argonne, Il, 1997.

${ }^{11}$ Forsberg, C. W., and Hopper, C. M. Definition of Weapons-Usable Uranium-233. ORNL/TM-13517. Oak Ridge National Laboratory, Oak Ridge, TN. 1998.

12 “RERTR,” URL: http://www.rertr.anl.gov/index.html [cited July 25th, 2013].

${ }^{13}$ Primm III, R. T., et al. Assumptions and Criteria for Performing a Feasibility Study of the Conversion of the High Flux Isotope Reactor Core to Use Low-Enriched Uranium Fuel. ORNL/TM-2005/269. Oak Ridge National Laboratory, Oak Ridge, TN. 2006.

14 “Fact Sheet," URL: http://nnsa.energy.gov/mediaroom/factsheets/reducingthreats [cited July 25 ${ }^{\text {th }}, 2013$ ].

${ }^{15}$ Davis, E. J., and Anderson, G. H. "The Incipience of Nucleate Boiling in Forced Convection Flow." AIChe Journal, Vol. 12, No. 4, 1966. Pp: 774-780.

${ }^{16}$ Blanco, P. J., Feijoo, R. A., and Urquiza, S. A. "A Unified Variational Approach for Coupling 3D-1D Models and its Blood Flow Applications." Computer Methods in Applied Mechanics and Engineering, Vol. 196, No. 41-44, 2007. Pp. 4391-4410.

${ }^{17}$ Gayer, M., Kortelainen, J., and Karhela T. "CFD Modeling as an Integrated Part of Multi-Level Simulation of Process Plants-Semantic Modeling Approach.” 2010 Summer Computer Simulation Conference, Society for Modeling and Simulation International, Vista, CA, 2010.

${ }^{18}$ Formaggia, L., et al. "On the Coupling of 3D and 1D Navier-Stokes Equations for Flow Problems in Compliant Vessels." Computer Methods in Applied Mechanics and Engineering, Vol. 191, No. 6-7, 2001. Pp. 561582 . 
19 Viel, A. "Strong Coupling of Modelica System-Level Models with Detailed CFD Models for Transient Simulation of Hydraulic Components in their Surrounding Environment." Proceedings 8th Modelica Conference, Modelica Association, Oberpfaffenhofen, Germany, 2011.

${ }^{20}$ Yang, F.-1., Zhang, X.-f., and Tan, G.-m. "One- and Two-Dimensional Coupled Hydrodynamics Model for Dam Break Flow.” Journal of Hydrodynamics, Vol. 19, No. 6, 2007. Pp. 769-775.

21 Marcer, R., et al. "Coupling 1D System AMESim and 3D CFD EOLE models for Diesel Injection Simulation." 23rd Annual Conference on Liquid Atomization and Spray Systems, Institute for Liquid Atomization and Spray Systems, Edwards AFB, CA, 2010.

${ }^{22}$ Morita, M., and Yen, B. "Numerical methods for conjunctive two-dimensional surface and three-dimensional sub-surface flows." International Journal for Numerical Methods in Fluids, Vol. 32, No. 8, 2000. Pp. 921-957.

23 Amara, M, Capatina-Papaghiuc, D., and Trujillo D. "Hydrodynamical Modeling and Multidimensional Approximation of Estuarian River Flows." Computing and Visualization in Science, Vol. 6, No. 2-3, 2004. Pp. 3946.

24 Steinebach, G, et al. "Mechanisms of Coupling in River Flow Simulation Systems." Journal of Computational and Applied Mathematics, Vol. 168, No. 1-2, 2004. Pp. 459-470.

${ }^{25}$ Lazure, P., and Dumas, F. “An External-Internal Mode Coupling for a 3D Hydrodynamical Model for Applications at Regional Scale (MARS).” Advances in Water Resources, Vol. 31, No. 2, 2008. Pp. $233-250$.

${ }^{26}$ Kilanehei, F., Naeeni, S., and Namin, M. "Coupling of 2DH-3D Hydrodynamic Numerical Models for Simulating Flow Around River Hydraulic Structures.” World Applied Sciences Journal, Vol. 53, No. 1, 2011. Pp. $63-77$.

${ }^{27}$ Zounemat-Kermani, M., and Sabbagh-Yazdi, S.-R. "Coupling of two- and three-dimensional hydrodynamic numerical models for simulating wind-induced currents in deep basins." Computers and Fluids, Vol. 39, No. 6, 2010. Pp. 994-1011.

${ }^{28}$ Gejadze, I., and Monnier, J. "On a 2D 'Zoom' for the 1D Shallow Water Model: Coupling and Data Assimilation." Computer Methods in Applied Mechanics and Engineering, Vol. 192, No. 45-48, 2007. Pp. 46284643 .

${ }^{29}$ Marin, J., and Monnier, J. "Superposition of Local Zoom Models and Simultaneous Calibration for 1D-2D Shallow Water Flows." Mathematics and Computers in Simulation, Vol. 80, No. 3, 2009. Pp. 547-560.

${ }^{30}$ Fernández-Nieto, E., Marin, J, and Monnier, J. "Coupling Superposed 1D and 2D Shallow-Water Models: Source Terms in Finite Volume Schemes.” Computers and Fluids, Vol. 39, No. 6, 2010. Pp. 1070-1082.

${ }^{31}$ Miglio, E., Perotto, S., and Saleri, F. "Model Coupling Techniques for Free-Surface Flow Problems: Part I." Nonlinear Analysis: Theory, Methods \& Applications, Vol. 63, No. 5, 2005. Pp. 1885-1896.

${ }^{32}$ Anglart, H, et al. "CFD Prediction of Flow and Phase Distribution in Fuel Assemblies with Spacers." Nuclear Engineering and Design, Vol. 177, No. 1-3, 1997. Pp: 215-228.

${ }^{33}$ Krepper, E., Končar, B., and Egorov, Y. "CFD Modeling of Subcooled Boiling-Concept, Validation and Application to Fuel Assembly Design.” Nuclear Engineering and Design, Vol. 237, No. 7, 2007. Pp: 716-731.

${ }^{34}$ Kocar, C., and Sokmen, C. "A CFD Model of Two-Phase Flow in BWRs with Results of Sensitivity and Uncertainty Analysis.” Nuclear Engineering and Design, Vol. 239, No. 10, 2009. Pp: 1839-1846. 
35 Lahey Jr., R. T., and Drew, D. A. "The Analysis of Two-Phase Flow and Heat Transfer Using a Multidimensional, Four Field, Two-Fluid Model." Nuclear Engineering and Design, Vol. 204, No. 1-3, 2001. Pp: 29-44.

${ }^{36}$ Končar, B., Kljenak, I., and Mavko, B. "Modeling of Local Two-Phase Flow Parameters in Upward Subcooled Flow Boiling at Low Pressure." International Journal of Heat and Mass Transfer, Vol. 47, No. 6-7, 2004. Pp: 1499-1513.

${ }^{37}$ Rohde, U., et al. "Fluid Mixing and Flow Distribution in a Primary Circuit of a Nuclear Pressurized Water Reactor-Validation of CFD Codes.” Nuclear Engineering and Design, Vol. 237, No. 15-17, 2007. Pp: 16391655 .

${ }^{38}$ Ion, S., et al. "Pebble Bed Modular Reactor The First Generation IV Reactor to Be Constructed." World Nuclear Association Annual Symposium, World Nuclear Association, London, England, 2003.

${ }^{39}$ Becker, S., and Laurien, E. "Three-Dimensional Numerical Simulation of Flow and Heat Transport in HighTemperature Nuclear Reactors.” Nuclear Engineering and Design, Vol. 222, No. 2-3, 2003. Pp: 189-201.

40 Shang, Z. "CFD Investigation of Vertical Rod Bundles of Supercritical Water-Cooled Nuclear Reactor." Nuclear Engineering and Design, Vol. 239, No. 11, 2009. Pp: 2562-2572.

${ }^{41}$ Johnson, R. W. "CFD Investigation of Experimental Data Proposed to be a Validation Data Set. Proceedings of the $17^{\text {th }}$ International Conference on Nuclear Engineering, ASME, New York, 2009.

${ }^{42}$ Baqué, F., et al. "Generation IV Nuclear Reactors - R\&D Program to Improve Sodium-Cooled Systems Inspection." First International Conference on Advancements in Nuclear Instrumentation Measurement Methods and their Applications, IEEE, Piscataway, NJ, 2010.

${ }^{43}$ Schulenberg, T., et al. "European Supercritical Water Cooled Reactor." Nuclear Engineering and Design, Vol. 241, No. 9, 2011. Pp: 3505-3513.

44 Ferng, Y. M., and Chen, C. T. "CFD Investigating Thermal-Hydraulic Characteristics and Hydrogen Generation from Graphite-Water Reaction after SG Tube Rupture in HTR-10 Reactor." Applied Thermal Engineering, Vol. 31, No. 14-15, 2011. Pp: 2430-2438.

45 Jeong, J.-J., et al. "Development of a Multi-Dimensional Thermal-Hydraulic System Code, MARS 1.3.1." Annals of Nuclear Energy, Vol. 26, No. 18, 1999. Pp. 1611-1642.

${ }^{46}$ Lee, S. Y., Jeong, J. J., and Kim, S. H. "COBRA/RELAP5: A Merged Version of the COBRA-TF and RELAP5/MOD3 Codes.” Nuclear Technology, Vol. 99, No. 2, 1992. Pp. 177-187.

${ }^{47}$ Aumiller, D. L., Tomlinson, E. T., and Bauer, R. C. "A coupled RELAP5-3D/CFD Methodology with a Proof-of-Principle Calculation.” 2000 International RELAP5 Users Seminar, International RELAP5 Users Group, Idaho Falls, ID, 2000.

${ }^{48}$ Walter, A., Schulz, A., and Lohnert, G. "Comparison of Two Models for a Pebbled Bed Modular Reactor Core Coupled to a Brayton Cycle.” Nuclear Engineering and Design, Vol. 236, No. 5-6, 2006. Pp: 603-614.

${ }^{49}$ Venter, P. J., and Mitchell, M. N. "Integrated Design Approach of the Pebble Bed Modular Reactor Using Models.” Nuclear Engineering and Design, Vol. 237, No. 12-13, 2007. Pp: 1341-1353.

${ }^{50}$ McCleary, J. Geometry from a Differential Viewpoint. $2^{\text {nd }}$ Ed. New York: Cambridge University Press, 1995.

${ }^{51}$ Litvin, F., and Fuentes, A. Gear Geometry and Applied Theory. $2^{\text {nd }}$ Ed. Cambridge: Cambridge University Press, 2004. 
${ }^{52}$ Cundy, H. M., and Rollett, A. P. Mathematical Models. $2^{\text {nd }}$ Ed. London: Oxford University Press, 1961. 2084.

${ }^{53}$ Jingzhou, X., and Zhang, X.-C. "Circular Involute Stage.” Optics Letters, Vol. 29, No. 17, 2004. Pp. 2082-

${ }^{54}$ Lockwood, E. H. A Book of Curves. London: Cambridge University Press, 1961.

${ }^{55}$ Gerstner, D., et al. "Safety Analysis Challenges with Irradiation of Reduced Enrichment for Research and Test Reactors (RERTR) Program Test Fuel Elements in the Advanced Test Reactor." EFCOG 2010 Safety Analysis Workshop, Energy Facility Contractors Group, Las Vegas, NV, 2010.

${ }^{56}$ Marshall, Frances M. Advanced Test Reactor Capabilities and Future Operating Plans. Idaho National Laboratory, Idaho Falls, ID. 2006.

57 “Core Description,” URL: http://web.mit.edu/nrl/www/reactor/core description.htm [cited August 1 ${ }^{\text {st }}, 2013$ ].

${ }^{58}$ Less, L. S. PreCalculus the Easy Way, $7^{\text {th }}$ Ed. Hauppauge, NY: Barron's Educational Series, 2005.

${ }^{59}$ Figliola, R. S., and Beasly, D. E. Theory and Design for Mechanical Instruments. $4^{\text {th }}$ Ed. Hoboken, NJ: John Wiley and Sons, Inc., 2006.

${ }^{60}$ Smith, K. Configuration Control of Software for HFIR Safety Basis Calculations. SBP-1300 Rev. 1. Oak Ridge National Laboratory, Oak Ridge, TN. 2008.

${ }^{61}$ Curtis, F., Ekici, K., and Freels, J. "Fluid-Structure Interaction Modeling of High-Aspect Ration Nuclear Fuel Plates Using COMSOL.” COMSOL Conference 2013 Boston, COMSOL AB, Boston, MA, 2013.

${ }^{62}$ Khane, V., Jain, P., and Freels, J. "Development of CFD Models to Support LEU Conversion of ORNL's High Flux Isotope Reactor.” ANS Winter Meeting, ANS, La Grange Park, IL, 2012.

${ }^{63}$ Wang, D., Jain, K., and Freels, J. “Application of COMSOL Pipe Flow Module to Develop a High Flux Isotope Reactor System Loop Model.” COMSOL Conference 2013 Boston, COMSOL AB, Boston, MA, 2013.

${ }^{64}$ Reddy, J. N., and Gartling, D. K. The Finite Element Method in Heat Transfer and Fluid Dynamics. $3^{\text {rd }}$ Ed. Boca Raton, FL: CRC Press, 2010.

${ }^{65}$ Amestoy, P. R., Duff, I. S., and L'Excellent, J.-Y. MUMPS MUltifrontal Massively Parallel Solver Version 2.0. Technical Report TR/PA/98/02. Centre Européen Recherche et de Formation Avancée en Calcul Scientifique, Toulouse, France. 1998.

${ }^{66}$ Gerald, C. F., and Wheatley, P. O. Applied Numerical Analysis. $7^{\text {th }}$ Ed. Boston, MA: Pearson Education, Inc., 2004.

${ }^{67}$ X-5 Monte Carlo Team, MCNP-A General Monte Carlo N-Particle Transport Code, Version 5. LA-CP-030245. Los Alamos National Laboratory, Los Alamos, NM. 2003.

${ }^{68}$ Incropera, F. P., et al. Fundamentals of Mass and Heat Transfer. $6{ }^{\text {th }}$ Ed. Hoboken, NJ: John Wiley \& Sons, Inc., 2007.

${ }^{69}$ Wilcox, D. C. Turbulence Modeling for CFD. $3^{\text {rd }}$ Ed. La Canada, CA: DCW Industries, 2006.

${ }^{70}$ Kays, W. M., and Crawford, M. E. Convective Heat and Mass Transfer. $3^{\text {rd }}$ Ed. New York: McGraw-Hill, 1993. 
${ }^{71}$ White, F. M. Viscous Fluid Flow. $3^{\text {rd }}$ Ed. New York: McGraw-Hill, 2006.

${ }^{72}$ COMSOL Documentation. v.4.3a. Boston, MA: COMSOL AB, 2012.

${ }^{73}$ Eckert, E. G., and Drake Jr., R. M. Heat and Mass Transfer. $1{ }^{\text {st }}$ Ed. New York: McGraw-Hill, 1959.

${ }^{74}$ Gambill, W. R., and Bundy, R. D. HFIR Heat-Transfer Studies of Turbulent Water Flow in Thin Rectangular Channels. ORNL-3079. Oak Ridge National Laboratory, Oak Ridge, TN. 1961. 


\section{Vita}

Adam Travis was born in Charlotte, NC, to Mike and Leigh Travis. He is the first of three children, followed by Chloe and Henry. After weathering the wrath of hurricane Hugo in Charlotte and spending a few years running from fire ants in Waco, TX, he moved to Morristown, TN, where he spent the bulk of his formative years. Upon graduation he enrolled as a civil engineer at the University of Tennessee because he'd been told he was "good with numbers" and that seemed like the kind of thing someone who was good with numbers would do. He switched to aerospace within three months, having discovered a budding fascination with flight and orbital mechanics. He was able to return to his hometown of Charlotte momentarily for a co-operative engineering assignment with US Airways throughout 2009-10. Adam took an undergraduate numerical analysis course in the spring of 2010 and in it he found his calling. He began working as an undergraduate research assistant with Dr. Kivanc Ekici in the subject matter the next semester. Adam attained a Bachelors of Science degree in aerospace engineering in May 2012. He spent the following summer at Oak Ridge National Laboratory (ORNL) as a graduate research intern with the Nuclear Engineering Science Laboratory Synthesis (NESLS) program. He continued the work begun in that internship in the pursuit of a Masters of Science degree in mechanical engineering which he attained in May 2014. 
ORNL/TM-2014/276

\section{INTERNAL DISTRIBUTION}

1. D. H. Cook (dhc@ornl.gov)

2. J. D. Freels (freelsjd@ornl.gov)

3. G. Ilas (ilasg@ornl.gov)

4. P. K. Jain (jainpk@ornl.gov

5. D. G. Renfro (renfrodg@ornl.gov)

6. K. A. Smith (smithka@ornl.gov)

7. G. L. Yoder Jr. (yodergljr@ornl.gov)

8. D. Chandler (chandlerd@ornl.gov)

9. $\quad$ E. L. Popov (popove@ornl.gov)

10. M. W. Kohring (kohringmw@ornl.gov)

11. ORNL/RRD Document Control (phillipsth@ornl.gov)

\section{EXTERNAL DISTRIBUTION}

12. I. T. Bodey, Savannah River Remediation, LLC., DWPF Mechanical Engineering Building: 704-25S Room 7 (Isaac.Bodey@,srs.gov)

13. R. V. Arimilli, Professor, Mechanical, Aerospace and Biomedical Engineering Department, 414 Dougherty Engineering Building, The University of Tennessee, Knoxville, Tennessee 37996-2210 (arimilli@utk.edu)

14. E. Fontes, COMSOL AB, Tegnergatan 23, SE-111 40, Stockholm, Sweden (ed@comsol.com)

15. W. F. Jones, Idaho National Laboratory, P.O. Box 1625, Idaho Falls, ID 83415-3750 (warren.jones@inl.gov)

16. K. T. Lowe, Bettis Atomic Power Laboratory, Bechtel Bettis, Inc, Mail Stop 36E, P. O. Box 79, West Mifflin, PA, 15122 (lowekt@bettis.gov)

17. A. E. Ruggles, Department of Nuclear Engineering, 315 Pasqua Engineering Building, Knoxville, TN 37996-2300 (aruggles@utk.edu)

18. B. Sjodin, COMSOL, Inc., New England Executive Park, Suite 350, Burlington, MA 01803 (bjorn@,comsol.com)

19. J. G. Stevens, Research and Test Reactors Department Manager, Nuclear Engineering Division, Argonne National Laboratory, 9700 S Cass Avenue, Bldg 208, Argonne, IL 60439 (johnstevens@anl.gov)

20. W. R. Marcum, Assistant Professor, Nuclear Engineering \& Radiation Health Physics, Oregon State University (marcumw@engr.orst.edu)

21. G. L. Solbrekken, Assistant Professor, Mechanical and Aerospace Engineering, E2411A Lafferre Hall, Columbia, MO, 65211 (SolbrekkenG@missouri.edu)

22. A. M. Tentner, Nuclear Engineering Division, Argonne National Laboratory, 9700 S Cass Avenue, Bldg 208, Argonne, IL 60439 (tentner@anl.gov)

23. K. Ekici, Assistant Professor, Graduate Program Director, Mechanical, Aerospace and Biomedical Engineering Department, 315 Perkins Hall, 415 Dougherty Engineering Building, The University of Tennessee, Knoxville, Tennessee 37996-2030 (ekici@utk.edu) 